
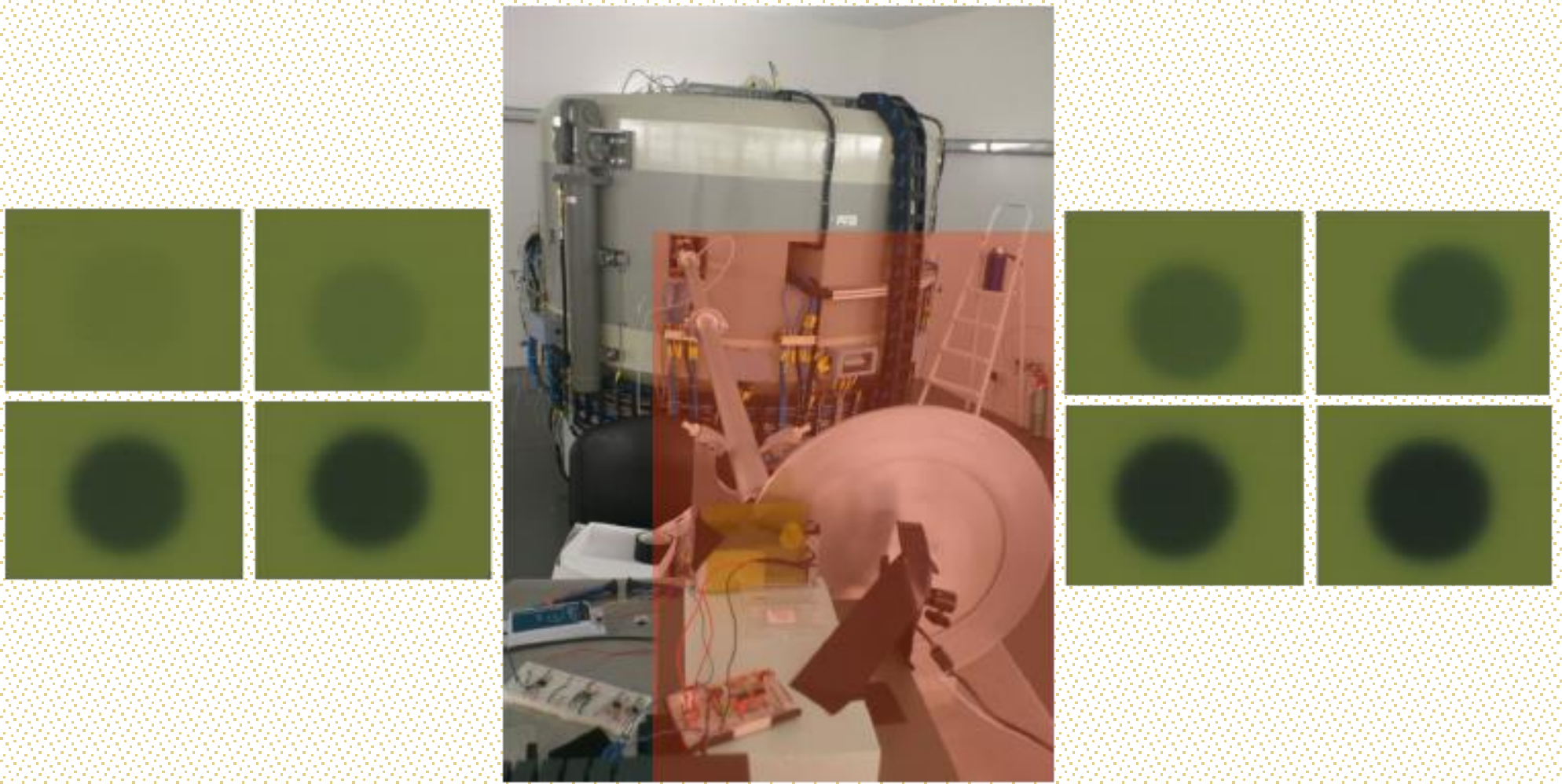

Sharif Hasan Mahmoud Ghithan

\title{
DEVELOPMENT OF A PET CYCLOTRON BASED IRRADIATION SETUP FOR PROTON RADIOBIOLOGY
}

Tese de Doutoramento em Engenharia Física, Especialidade de Instrumentação, orientada pelo Prof. Doutor Francisco Amaral Fortes de Fraga e pelo Prof. Doutor Paulo Alexandre Vieira Crespo e apresentada ao Departamento de Física da Faculdade de Ciências e Tecnologia da Universidade de Coimbra

Setembro de 2014

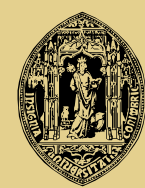

C 



\section{Development of a PET Cyclotron Based Irradiation Setup for Proton Radiobiology}

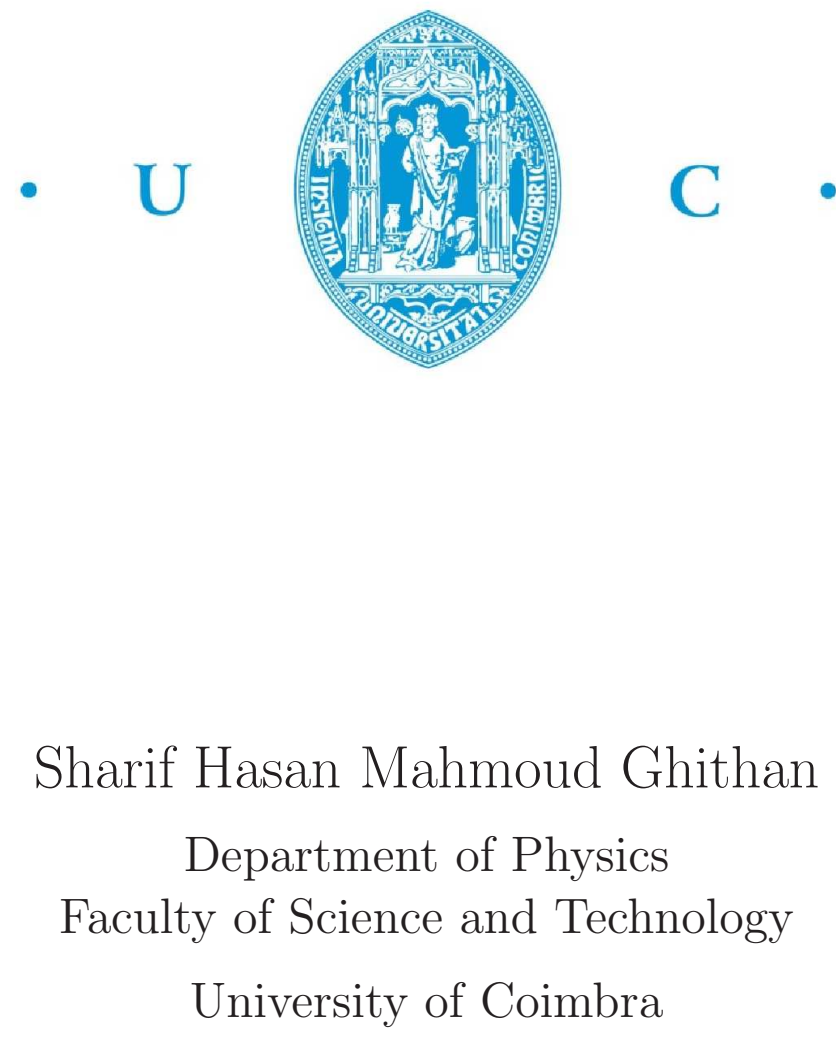

A thesis submitted for the degree of

Doctor of Philosophy in Physics Engineering

Coimbra - 2014 

I would like to dedicate this thesis to My loving parents

My beautiful wife

My lovely homeland "Palestine" 



\section{Contents}

Contents ii

List of figures

List of tables ix

List of acronyms and abbreviations xi

Resumo Xv

Overview Xvii

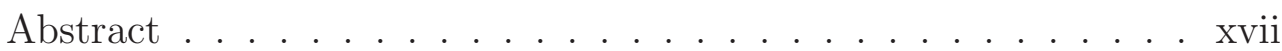

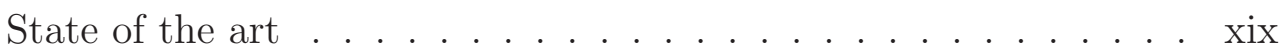

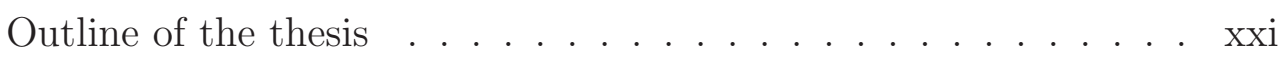

Thesis framework within the SESAME project . . . . . . . . xxii

1 Physics and biology of proton therapy 1

1.1 Introduction . . . . . . . . . . . . . . . . 1

1.2 Rationale for proton therapy . . . . . . . . . . . 7

1.2.1 Physical rationale for proton therapy ......... 7

1.2.1.1 Stopping power and the Bethe-Bloch formula . . 9 9

1.2.1.2 Absorbed dose ............... 11

1.2.1.3 Linear energy transfer (LET) . . . . . . . . . . . 11

1.2.1.4 Multiple Coulomb scattering and range straggling 13

1.2.2 Radiobiological rationale for proton therapy . . . . . . . 14

1.2.2.1 Biological effects of clustered DNA damage . . . 15

1.2.2.2 Relative biological effectiveness (RBE) . . . . . . 16

1.3 Advantages in comparison to photons . . . . . . . . . . . . 17

1.4 Advantages in comparison to other charged particles . . . . . . . 18

1.5 Beam transport systems . . . . . . . . . . . . . . . . . . 19

1.6 Beam shaping and dose delivery systems . . . . . . . . . . . . . . 20

1.6.1 Passive beam shaping . . . . . . . . . . . . . . 21 
1.6.2 Active beam shaping . . . . . . . . . . . . . . 21

2 State-of-the-art instrumentation for beam diagnostics 23

2.1 Introduction . . . . . . . . . . . . . . . . . . . 2. 23

2.2 Instrumentation for destructive beam diagnostics . . . . . . . 26

2.2 .1 Faraday cups . . . . . . . . . . . . . . . . . . 26

2.2 .2 Scintillation-based devices . . . . . . . . . . . . 27

2.2.2.1 Thin scintillator readout by PMT . . . . . . . . 29

2.2.2.2 Thin scintillator readout by CCD . . . . . . . 2. 29

2.2.2.3 Plastic scintillators . . . . . . . . . . . . . . 30

2.2 .3 Thermoluminescent dosimetry . . . . . . . . . . . . 32

2.2 .4 Film dosimetry . . . . . . . . . . . . . . . . . . 34

2.2.5 Semiconductor-based devices . . . . . . . . . . . . 38

2.2.5.1 Silicon-based semiconductor . . . . . . . . . . . 39

2.2.5.2 Diamond-based semiconductor . . . . . . . . 4 41

2.3 Instrumentation for non-destructive beam diagnostics . . . . . . . 43

2.3 .1 Transformers . . . . . . . . . . . . . . . . . . 43

2.3.2 Secondary electron monitors . . . . . . . . . . . . . 45

2.3.3 Secondary electron emission (SEM) grids . . . . . . . . . 47

2.3.4 Wire scanners . . . . . . . . . . . . . . . . . . . . . . 49

2.3.5 Gas-filled chambers . . . . . . . . . . . . . . . . . . . 49

3 Proton beam monitoring system at PET-dedicated cyclotrons 55

3.1 The PET cyclotron at the University of Coimbra . . . . . . . 55

3.1.1 Internal beam characteristics: Performance up to the beam port .................... 56

3.1.2 Beam external to the cyclotron radius . . . . . . . . . 59

3.2 Characterizing external beam parameters . . . . . . . . . . . 59

3.2 .1 Fluence . . . . . . . . . . . . . . . . . . . . . 60

3.2 .2 Beam energy . . . . . . . . . . . . . . . . . . . 61

3.2 .3 Bragg peak . . . . . . . . . . . . . . . . . 61

3.2 .4 Beam range . . . . . . . . . . . . . . . . . . . . . . 61

3.2 .5 Beam current . . . . . . . . . . . . . . . . . . . . 62

$3.2 .6 \quad$ Beam profile . . . . . . . . . . . . . . . . 62

3.2 .7 Dose and dose rate . . . . . . . . . . . . . . . 63

3.3 Preliminary characterization of the external proton beam from IC-

NAS cyclotron . . . . . . . . . . . . . . . . . . . . 65

3.3 .1 Motivation . . . . . . . . . . . . . . . . . 65

3.3.2 Out-of-yoke proton beam . . . . . . . . . . . . . . 65 65

3.3.3 Setup for the characterization of in-air beam divergence . . 66

3.3.3.1 Experiment at the PET cyclotron at ICNAS . . . 67 
3.3.3.2 Geant4 simulations .............. 70

3.3.3.3 Neutron and $\gamma$-ray dose contribution of an irradi-

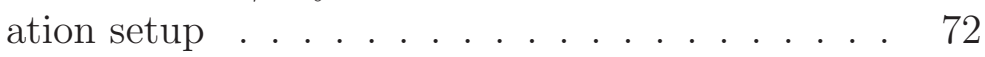

3.3.3.4 Assessing beam uniformity . . . . . . . . . . 74

3.4 Summary and outlook . . . . . . . . . . . . . 76

4 Fast and precise verification of proton beam position, range, and energy using a plastic scintillator at PET cyclotrons

4.1 Motivation . . . . . . . . . . . . . . . . . . . . . . 77

4.2 A 40-cm-long aluminum pipe . . . . . . . . . . . 78

4.2 .1 Setup description . . . . . . . . . . . . . . . . 78

4.2.2 Assessing proton Bragg peak . . . . . . . . . . . . . 78

4.3 A 2.4-m-long aluminum pipe . . . . . . . . . . . . . . . . . 81

4.3.1 Setup description . . . . . . . . . . . . . . . . . . 81

4.3.2 Assessing proton Bragg peak . . . . . . . . . . . . . . 82

4.3.3 Assessing initial energy of the proton beam . . . . . . . . 85

4.4 Discussion . . . . . . . . . . . . . . . . . . . . . . . . . . . . 89

4.5 Summary and outlook . . . . . . . . . . . . . . . . 89

5 On-line measurements of beam current from a PET cyclotron using a thin aluminum foil 91

5.1 Motivation ...................... . . 91

5.2 The emission of secondary electrons from a thin foil bombarded with protons ....................... 92

5.2.1 The emission of secondary electrons from a thin carbon foil bombarded with protons . . . . . . . . . . . 93

5.2.2 The emission of secondary electrons from a thin aluminum foil bombarded with protons . . . . . . . . . . . . 94

5.3 Assessing proton Bragg peak . . . . . . . . . . . . . . . . 94

5.4 Assessing beam current using a thin aluminum foil . . . . . . . 99

5.5 Assessing beam current using a thin carbon foil . . . . . . . . 101

5.6 Discussion . . . . . . . . . . . . . . . . . . . . . 103

5.7 Summary and outlook . . . . . . . . . . . . . . . 107

6 PET cyclotron based irradiation setup for proton radiobiology and radiophysiology 109

6.1 Motivation . . . . . . . . . . . . . . . . . . . . . . 109

6.2 Developments for an out-of-yoke irradiation setup . . . . . . . 110

6.2 .1 Out-of-yoke chopped beam . . . . . . . . . . . . . . 110

6.2.2 Hardware and software for dose and dose rate measurements 111

6.2.3 Controlled beam exposure via a ground-isolated power system 116 
6.2.3.1 Beam shutter . . . . . . . . . . . . . 117

6.2.3.2 Hardware for beam control . . . . . . . . . 117

6.3 Results . . . . . . . . . . . . . . . . . . . . . . . . . 119

6.3.1 Optimum cyclotron magnetic field for homogenous target

irradiation . . . . . . . . . . . . . . . 119

6.3.1.1 On target beam inhomogeneity with defocused

magnetic field . . . . . . . . . . . . 120

6.3.1.2 Explaining on target beam inhomogeneity . . . . 124

6.3.2 Current-based dose estimation using published film-response data . . . . . . . . . . . . . . 126

6.3.3 Assessing target dose by means of absolute 2D dosimetry

with Gafchromic ${ }^{\circledR}$ EBT2 film . . . . . . . . . . . . 129

6.3.3.1 X-ray film dosimetry . . . . . . . . . . . . 129

6.3.3.2 Validating proton dose based on X-ray film dosime-

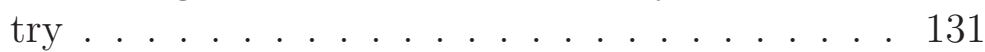

6.3.3.3 Estimating out-of-field dose . . . . . . . . . 132

6.3.3.4 Proton current-based dose versus optical-density dose . . . . . . . . . . . . . 135

6.4 A 2D position multi-well setup for proton radiobiology ..... 136

6.5 Summary and outlook . . . . . . . . . . . . . . 137

7 Conclusions and outlook 139

\section{Appendices}

A Havar ${ }^{\circledR}$ window 143

B Geant4 simulation toolkit 145

B.1 Introduction . . . . . . . . . . . . . . . . . . . . 145

B.2 Monte-Carlo validation . . . . . . . . . . . . . . . . 146

C Frequency-to-voltage converter 149

References 153

Acknowledgements 165 


\section{List of figures}

1 Photograph of the microtron in the SESAME experimental hall . xxiii

2 Scheme of SESAME machine and the beamlines . . . . . . . . . xxiv

1.1 Present situations of the different treatment methods against cancer

1.2 Particle therapy evolution since the discovery of the X-ray . . . . 4

1.3 Depth-dose profiles for photons, protons, and carbon ions . . . . 8

1.4 Charged particles have an advantageous radiation . . . . . . 8

1.5 Energy loss in water for several ions . . . . . . . . . . . . . . 10

1.6 Beam width at different depths in water . . . . . . . . . . . 14

1.7 Schematic figure of radiation damage to DNA . . . . . . . . . . 15

1.8 Definition of RBE, the relative biological effectiveness . . . . . . . 17

1.9 An IBA proton beam delivery system . . . . . . . . . . . . . . . 20

1.10 Sketch of a fully passive beam shaping system . . . . . . . . . . 22

1.11 Schematic view of the active beam scanning treatment modality . 22

2.1 Left: Schematic diagram of a Faraday cup . . . . . . . . . 27

2.2 Schematic diagram of a plastic scintillation counter . . . . . . . . 30

2.3 Schematic diagram (left) and photograph (right) of an intercepting 30

2.4 Screen holder with three screens . . . . . . . . . . . . . . . 31

2.5 A simple model of energy absorption in a TL material . . . . . . . 33

2.6 Schematic diagram of a simple TLD readout system . . . . . . . . 34

2.7 Configuration of Gafchromic ${ }^{\circledR}$ EBT (left) and EBT2 (right) . . . 37

2.8 Simplified energy band structure diagrams for insulators . . . . . 39

2.9 Schematic diagram of semiconductor detector . . . . . . . . . . . 40

2.10 Sketch of a simple diamond-based detector . . . . . . . . . . . 42

2.11 Schematic view showing the magnetic field induced by a charged . 44

2.12 Schematic view of a current transformer for pulsed beams . . . . . 44

2.13 Photograph of DC current transformer (DCCT) being used at LHC 46

2.14 Schematic diagram (left) and photograph (right) of a secondary . 47

2.15 Photograph of a SEM grid made of thin ribbons . . . . . . . . 47

2.16 Scheme shows a SEM grid consisting of an array of sensing wires . 48 
2.17 Schematic view shows a conventional BPM (beam position monitor) 49

2.18 Pendulum scanner or "flying wire" being used at CERN . . . . . 50

2.19 Schematic diagram of a parallel plate ionization chamber . . . . . 50

2.20 Diagram of ionization chamber . . . . . . . . . . . . 51

2.21 Variation of pulse height produced by different types of detectors 52

2.22 Schematic diagram of a parallel plate type free air ionization chamber 54

3.1 Photograph of the Cyclone ${ }^{\circledR} 18 / 9$-HC cyclotron . . . . . . . 56

3.2 Schematic view representing extraction by stripping . . . . . . 57

3.3 Schematic view of one of the exit ports of the Cyclone ${ }^{\circledR}$ 18/9-HC 57

3.4 Depth-dose profile for proton beam in a medium . . . . . . . . 62

3.5 Schematic diagram (left) and photograph (right) of the 40-cm-long 66

3.6 Experimental setup (left) and photograph (right) at ICNAS . . . 67

3.7 Experimental results obtained with three EBT2 Gafchromatic ${ }^{\circledR}$. 67

3.8 In-air beam profile obtained with three Gafchromic ${ }^{\circledR}$ EBT2 films 68

3.9 Saturated, proportionality, and no-response regions . . . . . . . . 69

3.10 Schematic diagram of the irradiated area for the three EBT2 films 69

3.11 Two views (side view (left) and top view (right)) of the experimental 71

3.12 Simulation results obtained with the three EBT2 . . . . . . . 71

3.13 Two views (side view (left) and top view (right)) of the irradiation 73

3.14 Simulation results obtained with EBT2 films . . . . . . . . . 73

3.15 Two photographs before (left) and after (right) irradiation . . . . 74

3.16 Simulation results obtained for nine EBT2 Gafchromatic ${ }^{\circledR}$ films . 75

3.17 Experimental results obtained with three EBT2 films . . . . . . 75

4.1 Left: Scheme of the experimental setup for the first plastic . . . . 79

4.2 Longitudinal (left) and lateral (right) profiles for the measured . . 80

4.3 Simulation results for $15.45 \mathrm{MeV}$ protons in a block . . . . . . . . 81

4.4 Schematic diagram of the 2.4-m-long aluminum pipe . . . . . . . 81

4.5 Scheme of the experimental setup for the second plastic scintillator 83

4.6 Five photographs for the plastic scintillator for the setup . . . . . 83

4.7 Longitudinal (left) and lateral (right) beam profiles . . . . . . . . 84

4.8 Left: Lateral beam profile at the Bragg peak and at the plateau . 85

4.9 Scheme of the experimental setup for the third plastic scintillator 86

4.10 Left: Two photographs for the plastic scintillator for the setup . . 86

4.11 Simulation results for the experimental setup . . . . . . . . . 87

4.12 Seven photographs for the plastic scintillator for the setup . . . . 87

4.13 Time variation of the proton beam range (left) and of the intensity 88

4.14 Two photographs for the distorted scintillator piece . . . . . . . . 88

5.1 Schematic diagram of the experimental setup of the stacked target 95 
5.2 Schematic diagram of the in-house made electronics . . . . . . . 95

5.3 Signals from the first three aluminum foils . . . . . . . . . . . . 97

5.4 Signals from the last three aluminum foils . . . . . . . . . . . 97

5.5 Simulation results for the experimental setup of the stacked target 98

5.6 Secondary current with error bars as a function of foil number . . 98

5.7 Schematic diagram of the experimental setup of the aluminum foil 99

5.8 Schematic diagram of the in-house made electronics . . . . . . . . 100

5.9 AC analysis of the output voltage . . . . . . . . . . . . . 101

5.10 Signals from the aluminum foil . . . . . . . . . . . . . 102

5.11 Four photographs showing the plastic scintillator . . . . . . . . 102

5.12 Schematic diagram of the experimental setup of the carbon foil . . 103

5.13 Calibration curve for secondary current measurements . . . . . . . 104

5.14 Calculated dose rate as a function of the measured secondary current 106

6.1 Schematic diagram of the irradiation setup . . . . . . . . . . 111

6.2 Schematic view of the beam duty cycle . . . . . . . . . . . 112

6.3 Schematic diagram of the out-of-yoke irradiation setup . . . . . . 113

6.4 Schematic diagram of an in-house made transimpedance electronics 114

6.5 Left: Schematic diagram of the experimental setup . . . . . . . . 116

6.6 Two photographs of a computer-controlled shutter . . . . . . . . 117

6.7 Schematic diagram of in-house developed electronics systems . . . 118

6.8 Measured dose rate as a function of magnet current . . . . . . . . 120

6.9 Experimental results obtained with two stacked targets . . . . . . 121

6.10 Proton beam profiles for the beam spots . . . . . . . . . . . . 121

6.11 Left: A scan copy of an irradiated EBT2 film with focused protons 122

6.12 Proton beam profiles obtained for two beam spots . . . . . . . . . 123

6.13 Measured Bragg peak for focused (left) and defocused (right) proton 124

6.14 Schematic view of the stacked target . . . . . . . . . . . . . 124

6.15 A view of the experimental setup of the stacked target experiment 125

6.16 Simulation results obtained with the sixth EBT2 film . . . . . . 125

6.17 Experimental results obtained with 10 EBT2 films . . . . . . . . 127

6.18 Absolute dose (X-ray dose) in the irradiated EBT2 calibration films 128

6.19 Left: Relative pixel color for the irradiated EBT2 films . . . . . . 128

6.20 Experimental results obtained with an EBT2 film . . . . . . . . . 130

6.21 Left: Absolute dose (optical-density dose) in the irradiated EBT2 132

6.22 Experimental results obtained with an EBT2 film . . . . . . . . 132

6.23 Left: A scan copy of an irradiated EBT2 film . . . . . . . . . . 133

6.24 Geant4 simulation results obtained for proton spectrum . . . . . . 134

6.25 Two top views of the irradiation setup simulated by means of Geant4135

6.26 Current-based dose as a function of optical-density dose . . . . . . 136

6.27 Schematic diagram of a 24-cavity cell culture plate control system 137 
B.1 View of the experimental setup of Verbinski and Burrus experiment 147

B.2 Double differential cross sections $\left(\mathrm{d}^{2} \sigma /(\mathrm{dEd} \theta)\right) \ldots \ldots \ldots \ldots$

C.1 Schematic diagram of the frequency-to-voltage converter . . . . 150

C.2 Signals from the frequency-to-voltage converter . . . . . . . 150

C.3 Schematic diagram of the in-house made electronics . . . . . . 151 


\section{List of tables}

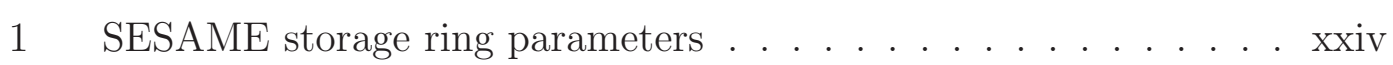

1.1 World cancer statistics for the most common cancers . . . . . . 2

1.2 Proton therapy facilities in operation in 2013 (A) . . . . . . 5

1.3 Proton therapy facilities in operation in 2013 (B) . . . . . . . 6

1.4 Carbon therapy facilities in operation in $2013 \ldots$. . . . . . . 6

1.5 Variables and values of the Bethe-Bloch equation . . . . . . . . 10

1.6 Typical LET values of ionizing radiation . . . . . . . . . . . . . . 12

1.7 General cost estimation of radiation therapy . . . . . . . . . . 18

2.1 Properties of common inorganic scintillators . . . . . . . . . 31

2.2 Nominal atomic abundances of Gafchromic ${ }^{\circledR}$ EBT2 film . . . . . 37

2.3 Properties of silicon and diamond crystals . . . . . . . . . 43

2.4 Ionization potentials $I_{e}, \mathrm{~W}$-values, stopping powers $(d E / d x) \ldots 53$

3.1 Different parameters of the Cyclone ${ }^{\circledR}$ 18/9 -HC cyclotron . . . . 58

3.2 Radiation weighting factor $W_{T}$ for different types of particles . . . 64

3.3 Tissue weighting factors $W_{T}$ according to 1990 recommendations . 64

3.4 The flux density values $\phi$ at the locations of the three EBT2 films 70

5.1 Measured and calculated values from the aluminum foil experiment 106

A.1 Physical properties of Havar ${ }^{\circledR}$ alloy . . . . . . . . . . . . . 143

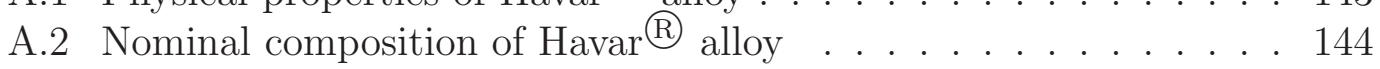


List of tables 


\title{
List of acronyms and abbreviations
}

\author{
2D / 3D Two Dimension / Three Dimension \\ AC Alternating Current \\ ADC Analog-to-Digital Converter \\ ATIMA Atomic Interaction with Matter \\ BESSY Berlin Electron Storage Ring Society for Synchrotron Radiation, Germany, \\ originally named Berliner Elektronenspeicherring-Gesellschaft für \\ Synchrotronstrahlung \\ BNC Bayonet Neill Concelman \\ BPM Beam Position Monitor \\ CAMAC Computer A Automated Measurement and Control \\ CCD Charge-Coupled Device \\ CERN European Organization for Nuclear Research, Geneva, Switzerland, \\ originally named Conseil Européene pour la Recherche Nucléaire \\ CF $\quad$ ConFlat; Control Film \\ DAC Digital-to-Analog Converter \\ DC Direct Current \\ DCCT DC Current Transformer \\ DNA Deoxyribonucleic Acid \\ DR Dose Rate \\ EBT External Beam Therapy \\ ENET EtherNET \\ EUR Euro
}


FCT The Portuguese Foundation for Science and Technology, Lisbon, Portugal, originally named Fundação para a Ciência e a Tecnologia

FWHM Full Width at Half Maximum

Geant4 Geometry and tracking 4

GPIB General Purpose Interface Bus

GSI The German Society for Heavy Ions Research, Darmstadt, Germany, originally named Gesellschaft für Schwerionenforschung

HC $\quad$ High Current

HIT The Heidelberg Ion-Beam Therapy Center, Heidelberg, Germany, originally named Heidelberger Ionenstrahl-Therapiezentrum

HPC $\quad$ High Performance Computing

IBA Ion Beam Application

ICNAS Portuguese Institute for Nuclear Sciences Applied to Health, University of Coimbra, Portugal, originally named Instituto de Ciências Nucleares Aplicadas à Saúde

ICRU International Commission on Radiation Units and Measurements, Inc., Bethesda, MD, USA

ISP International Specialty Products

LET Linear Energy Transfer

LHC Large Hadron Collider

LINAC Linear Accelerator

LIP Laboratory of Instrumentation and Experimental Particle Physics, University of Coimbra, Portugal, originally named Laboratório de Instrumentação e Física Experimental de Partículas

MC Monte Carlo

MCS Multiple Coulomb S Scattering

PC Personal Computer

PET Positron Emission Tomography

PMT Photomultiplier Tube

PSI $\quad$ Paul Scherrer Institute, Villigen, Switzerland

PTW Physikalisch-Technische Werkstättena

PV $\quad$ Pixel Value

RBE Relative Biological Effectiveness

RF $\quad$ Radiofrequency 


\section{ROI Region of Interest}

SEM Secondary Electron Emission

SESAME Synchrotron-light for Experimental Science and Applications in the Middle East, Allan, Jordan

SOBP $\quad$ Spread-out Bragg Peak

SRIM $\quad$ Stopping and Range of Ions in Matter

TL Thermoluminescent

TLD Thermoluminescent Detector

TRIM Transport of Ions in Matter

UNESCO United Nations Educational, Scientific, and Cultural Organization UV Ultraviolet

WHO Wold Health Organization

Y $\quad$ Yield


List of acronyms and abbreviations 


\section{Resumo}

O número de ciclotrões com capacidade para acelerar protões até cerca de $20 \mathrm{MeV}$ tem vindo a aumentar em todo o mundo. Apesar de o objectivo principal das instalaçõoes que contêm estas máquinas ser a produção de radionuclídeos para tomografia por emissão de positrões (PET, do inglês positron emission tomography), algumas dessas instalações estão equipadas com várias linhas de feixe que podem ser adaptadas para investigação científica. Por exemplo, radiobiologia, radiofisiolgia e outros estudos de dosimetria podem ser realizados utilizando uma destas linhas de feixe devidamente adaptada para o efeito. Neste trabalho, uma das linhas de feixe do ciclotrão PET da Universidade de Coimbra foi complementada e instrumentada por forma a possibilitar a irradiação de um arranjo experimental com um feixe de protões de elevada qualidade. Esta nova linha de feixe foi construída de raíz e sem causar qualquer interferência com as demais linhas do ciclotrão, dedicadas à produção de radionuclídeos. São apresentados resultados tanto experimentais como de simulação, estes últimos obtidos através dos pacotes de simulação SRIM/TRIM e Geant4, tendo como objectivo a medição do pico de Bragg depositado pelo feixe de protões do ciclotrão PET, com uma energia nominal de $18 \mathrm{MeV}$. Utilizando um bloco de plástico cintilador e uma câmara fotográfica com ligação à internet da marca D-link foi possível medir tanto o alcance como a largura do feixe de protões com uma resolução espacial inferior a $0,1 \mathrm{~mm}$. Os alcances do feixe medidos após este passar pelo meio de um tubo de alumínio com vácuo com $40 \mathrm{~cm}$ de comprimento e um segundo tubo com 2,4 m de comprimento permitiram confirmar que a energia efectiva do feixe é de $18 \mathrm{MeV}$. Mediu-se também o pico de Bragg utilizando um alvo constituído por várias folhas de alumínio intercaladas com lâminas de polietileno. O sinal de corrente foi recolhido das várias folhas de alumínio através de amplificadores de transimpedância fabricados no âmbito desta tese. Verificou-se que o pico de Bragg assim medido é consistente com simulações realizadas utilizando o pacote SRIM/TRIM.

Após a instalação da linha de feixe no perímetro exterior do ciclotrão, esta foi caracterizada, calibrada e validada. Para tal, o sinal induzido pela passagem do feixe por uma folha de alumínio com $20 \mu \mathrm{m}$ de espessura é lido através do 
amplificador de transimpedância mencionado. Este sinal amplificado providencia informação de dose em tempo real através de um programa desenvolvido em $\mathrm{C} / \mathrm{C}++$. Para além da dose, as principais variáveis de monitorização que este programa providencia incluem a corrente do feixe, a carga integrada em conjunto com a taxa de dose. Deste modo a dose e a corrente integrada (carga total) entregue até um dado instante na montagem experimental pode ser controlada por meio de um obturador controlado por computador. Feixes de protões com correntes tão baixas como $10 \mathrm{pA}$ podem deste modo ser aferidas. A folha de alumínio foi escolhida por ser resistente à radiação, possuir baixa densidade e baixa probabilidade de radioactividade induzida pelo feixe e, finalmente, por representar um custo negligenciável. Junta-se a estas vantagens o facto de o método potenciar o cálculo da dose entregue a um alvo durante uma irradiação, com uma perda mínima da energia do feixe de protões, e com dispersão igualmente mínima.

Resultados experimentais e simulações com o Geant4 são apresentados que revelam a aplicação, pela primeira vez, de um feixe de $18 \mathrm{MeV}$ proveniente de um ciclotrão para irradiação de uma região seleccionada de um alvo. Fazendo uso do sistema de dosimetria apresentado no parágrafo anterior foi possível irradiar de modo homogéneo uma região circular com $18 \mathrm{~mm}$ de diâmetro. Torna-se assim possível irradiar culturas celulares localizadas em placas multi-poços com um diâmetro por poço de $16 \mathrm{~mm}$, como é usual em experiências de radiobiologia. Verificou-se que o controlo do campo magnético aplicado dentro do ciclotrão é crucial para se obter uma irradiação uniforme em todo o campo do alvo. Para tal, efetua-se antes de cada irradiação e com o obturador fechado, um varrimento à corrente que gera o campo magnético dentro do ciclotrão, medindo-se um perfil quase gaussiano e tomando-se o seu valor central para se obter uma irradiação homogénea. As taxas de dose no alvo (entre $500 \mathrm{mGy} / \mathrm{s}$ e $5 \mathrm{mGy} / \mathrm{s}$ ) são obtidas através de um disco em rotação posicionado na trajectória do feixe. O disco, com $150 \mathrm{~mm}$ de raio e uma fenda de $0,5 \mathrm{~mm}$ na sua extermidade, permite reduzir a taxa de dose por um factor de $5 \times 10^{-4}$. Finalmente, vários filmes do tipo Gafchromic ${ }^{\circledR}$ EBT2 foram expostos a diferente valores de dose por forma a validar toda a instalação para irradiação de um alvo com feixes de protões. Para tal validação fez-se uso do sistema de dosimetria em filme 2D do Serviço de Radioterapia do Centro Hospitalar Universitário de Coimbra. A dose absoluta nos filmes irradiados com protões foi verificada neste sistema e apresentou uma precisão melhor que $2 \%$.

Palavras-chave: Sistemas de monitorização e controlo online; Dosimetria; Instrumentação para ciclotrões; Emissão secundária de electrões; Radiobiologia; Simulação SRIM/TRIM; Simulação Geant4. 


\title{
Overview
}

\begin{abstract}
The number of cyclotrons capable of accelerating protons to about $20 \mathrm{MeV}$ is increasing throughout the world. Originally aiming at the production of positron emission tomography (PET) radionuclides, some of these facilities are equipped with several beamlines suitable for scientific research. Radiobiology, radiophysiology, and other dosimetric studies can be performed using these beamlines. In this work, a PET cyclotron was fitted with a long beam transport line to irradiate with a good quality proton beam experimental setups. The beamline was configured as a natural extension of one of the cyclotron beam ports, while keeping available the other beam ports for PET radionuclides production. Experimental results are reported, together with SRIM/TRIM and Geant4 simulations, which aim at measuring the Bragg peak of the $18-\mathrm{MeV}$ proton beam from the PET cyclotron. By using a piece of plastic scintillator and a D-link Ethernet-based camera, the proton beam range and width were measured with a spatial resolution of $0.1 \mathrm{~mm}$. The ranges of the proton beam in the plastic scintillator were used to assess its energy after trespassing one or two Havar ${ }^{\circledR}$ windows and either a 40-cm-long or a 2.4-m-long aluminum pipe. The initial energy of the proton beam from the PET cyclotron was found to be $18 \mathrm{MeV}$. Additionally, the Bragg peak of the protons from the PET cyclotron was assessed using a stacked target consisting of several aluminum foils interleaved with polyethylene sheets, readout by in-house made transimpedance electronics. The measured Bragg peak is consistent with simulations performed using the SRIM/TRIM simulation toolkit.

An out-of-yoke irradiation setup using the accelerated proton beam coming from the PET cyclotron was developed, characterized, calibrated, and validated. A $20-\mu \mathrm{m}$-thick aluminum transmission foil is readout by in-house made transimpedance electronics, providing online dose information via a $\mathrm{C} / \mathrm{C}++$ program.
\end{abstract}


The main monitoring variables include beam current, integrated charge together with dose rate. The beam monitor is able to readout and deliver these variables in real-time. Hence the dose and integrated current (total charge) delivered upto a given instant to an experimental setup may be controlled via a computercontrolled shutter that was installed in the beam path. Proton beam currents down to $10 \mathrm{pA}$ can be assessed using the thin aluminum foil. The aluminum was chosen for this task because it is radiation hard, it has low density and low radiation activity, and finally because it is easily available at negligible cost. In addition, this method allows for calculating the dose delivered to a target during an irradiation with high efficiency, and with minimal proton energy loss and scattering.

Experimental results and Geant4 simulations are reported, which aim at using for the first time the $18 \mathrm{-MeV}$ proton beam from a PET cyclotron to irradiate a selected region of a target using the developed dosimetry system. By using this system, a homogeneous beam spot on target with a diameter of $18 \mathrm{~mm}$ can be controlled. This allows controlled irradiation of cell cultures located in typical biological multi-well dishes with diameters of $16 \mathrm{~mm}$ each. It was found that the control of the magnetic field applied inside the cyclotron plays a major role for achieving said homogeneity. For that, scanning the magnet current and measuring the corresponding dose rate reveals a quasi-Gaussian shaped curve that must be known before any irradiation procedure (the final shutter is closed during such measurements). The optimum magnet current is taken from the center of the Gaussian-shaped curve, hence producing a homogenous dose on target. The measured dose rate on target ranges from $500 \mathrm{mGy} / \mathrm{s}$ down to $5 \mathrm{mGy} / \mathrm{s}$. This is achieved with a $150 \mathrm{~mm}$ radius rotating disk with a slit of $0.5 \mathrm{~mm}$ width, that decreases target dose rates by a factor of $5 \times 10^{-4}$. Several Gafchromic ${ }^{\circledR}$ EBT2 films were exposed to different values of dose for validating the developed irradiation setup using the 2D film dosimetry system of the Department of Radiotherapy of Coimbra University Hospital Center. The absolute dose in the irradiated films were assessed with a precision better than $2 \%$. It is planned, in the near future, to irradiate small animals, cell cultures, or other materials or samples.

Keywords: Control and monitor systems online; Dosimetry; Instrumentation for cyclotrons; Secondary-electron emission; Radiobiology; SRIM/TRIM simulation; Geant4 simulation. 


\section{State of the art}

The number of proton and heavy ion radiotherapy facilities is increasing throughout the world, with about forty centers currently operational and more than twenty in a planning stage or already under construction. Proton and heavy ion therapy outcomes are triggering the interest of more and more medical facilities, of a crescent number of countries, in making them available for their patients. At the same time, cyclotrons capable of accelerating protons up to about $20 \mathrm{MeV}$ are being worldwide installed. Although the purpose of the latter is mainly positron emission tomography (PET) radioisotope production, they are equipped with several beamlines suitable for scientific research, rendering such proton beam ports extremely useful for radiobiological and radiophysiological experiments.

In Portugal, an IBA (Ion Beam Applications, SA) model Cyclone ${ }^{\circledR}$ 18/9 -HC (high current) cyclotron was installed at ICNAS - Instituto de Ciências Nucleares Aplicadas à Saúde, University of Coimbra, for positron emission tomography in 2010. Such facility is equipped with eight beamlines. Each beamline may deliver proton currents up to $150 \mu \mathrm{A}\left(1 \times 10^{15}\right.$ particles/s $)$. Radiobiology, radiophysiology, and dosimetric studies, among others (e.g., space instrumentation tests) can be performed using these beamlines. Radiobiological and radiophysiological studies using proton beams from the PET cyclotron may contribute to further improve proton therapy results, namely by giving input to pertinent scientific questions such as: (1) the impact of fractionation of particle radiotherapy on dose effectiveness, including different effects in regions irradiated in the Bragg peak versus the plateau [Debus et al., 2003]; (2) the influence of living tissue responses to the radiotherapeutic injury, such as edema, tumor growth/shrinkage, vascular damage with consequent increased tissue permeability, and inflammatory processes with consequent changes in tissue densitv and stoichiometry Denham and Hauer-Jensen, 2002]; (3) the adjuvant and/or toxic effects of using natural [Drisko et al., 2003] or pharmaceutical compounds in concomitance with radiotherapy (RT); (4) the causes of hypersensitivity to low doses of radiation, a phenomenon called low-dose hyper-radiosensitivity [Schettino et al., 2010; Steel, 2007]; (5) the spatial and temporal evolution of radiation damage, which can be evaluated through the spatial and temporal evolution of DNA (deoxyribonucleic acid) single. double [Schettino et al..2010]. and clustered strand breaks [Elsässer et al., 2010; Schardt et al., 2010]; (6) the beneficial (adaptive response, hormesis) and/or cancer-inducing effect of low-level radiation to living cells, requiring precise delivery of cGy [Wolff, 1998] to tens of cGy [Feinendegen, 2005], as well as the determination of whether a stochastic/deterministic threshold dose takes place, and following which dose-response model, e.g., linear-quadratic, linear- 
threshold, linear-non-threshold, supra-linear, quadratic, or hormetic; and (7) the mechanisms underlying the radiation-induced bystander effect [Mothersill and Seymour, 2004; Widełet al., 2009]. Input to these and other issues shall contribute to further improving radiotherapy outcomes and potentially decreasing of mortality rates in cancer patients.

This line of research aims at developing an instrument for quantifying and controlling in real time a proton beam at the PET cyclotron at ICNAS. The cyclotron has been fulfilling, at the topmost level, its initial design goals: the production of state-of-the-art/start-of-the-art radionuclide tracers for medical imaging, namely positron emission tomography for disease staging and fundamental science in oncology, neurology, and cardiovascular pathologies. Within this line of research, one of the beamlines of the cyclotron was identified as being capable of providing scientific input to other areas of fundamental science with potential high impact to society, namely radiotherapy and radiation protection. Radiotherapy studies (radiobiology and radiophysiology) make use of relatively high dose values (few Gy to tens of Gy) with curative intent for oncologic disease, among others. In radioprotection the dose levels are very low (100 mGy or lower) and the scientific goal is understanding radiobiological/radiophysiological carcinogenic responses to such low levels of radiation.

Without disturbing the working cycles allocated for PET science, one dedicated beamline of the proton PET cyclotron was used to instrument a selfdeveloped system capable of monitoring beam variables, and of controlling the dose on target based on a computer application located outside the bulky cyclotron bunker (2-m-thick concrete walls). 


\section{Outline of the thesis}

This manuscript, Development of a PET Cyclotron Based Irradiation Setup for Proton Radiobiology, is organized in seven chapters. Chapter 1 starts with a brief introduction about cancer incidence and mortality. A general introduction on particle radiation therapy follows, with emphasis on the physical and biological rationales for the use of ions (e.g., protons) compared to conventional radiation (e.g., photons). Finally, the remainder of the chapter reviews the beam shaping and dose delivery systems being used in ion therapy facilities. Chapter 2 starts with a general introduction about instrumentation for beam diagnostics. Then, the destructive and non-destructive methods for beam monitoring are presented, respectively. The principle of operation and the advantages and disadvantages of each instrument are given. Chapter 3 begins with a description of the cyclotron installed at the University of Coimbra in Instituto de Ciências Nucleares Aplicadas à Saúde (ICNAS). The beam parameters that should be measured are presented later. The chapter ends with a preliminary experimental and Geant4 simulation results about the characterization of the proton beam achievable outside the yoke of the PET cyclotron from the University of Coimbra. Chapter 4 shows experiments with a plastic scintillator. These experiments were performed at ICNAS in order to assess the proton range after trespassing one or two Havar ${ }^{\circledR}$ windows and either a 40-cm-long or a 2.4-m-long aluminum pipe. The initial energy of the proton beam from the PET cyclotron was also evaluated. The fifth and sixth chapters of the manuscript are the main contributions and each of them corresponds to a scientific article. Chapter 5 describes a technique used for on-line measuring the beam current from a PET cyclotron using a thin aluminum foil. Simulations performed using the SRIM/TRIM toolkit in order to assess the proton Bragg peak and initial energy are also presented. On the other hand, chapter 6 describes the design, characterization, calibration, and validation of an out-of-yoke irradiation setup that was assembled and installed at the PET-dedicated cyclotron at ICNAS. The conclusions from this thesis, together with future work suggestions, are presented in chapter 7 . It recalls the context, objectives, and achievements of this work. 


\section{Thesis framework within the SESAME project}

SESAME - Synchrotron-light for Experimental S Science and Applications in the Middle East

As in daily life, in scientific research we learn by seeing things using light, except that researchers use light that ranges beyond the visible, in the infrared and the ultraviolet, to X-rays and beyond. The extraordinary power of synchrotron light, which is one of the advanced sources of light, has made it an essential tool for studying matter on scales ranging from biological cells to atoms. It has a huge impact in fields that include archaeology, biology, chemistry, environmental science, geology, medicine, and physics. Therefore, it is important to build a synchrotron light source in the Middle East to promote regional cooperation in science, solidarity, and peace.

The SESAME project is under constructing in the Middle East as a first major international research center. It is located in Allan, Jordan (30 km from Amman). The SESAME project aims to foster outstanding science and technology in the Middle East and neighbouring countries and it is doing this with steadfast support from the united nations educational, scientific, and cultural organization (UNESCO). It will build scientific and technical capacity and facilitate collaboration between the region's scientists and engineers. It involves at present the following member states: Bahrain, Cyprus, Egypt, Iran, Israel, Jordan, Pakistan, Palestine, and Turkey. In addition to Members, SESAME also has some observer countries, among which a number of countries from Europe (France, Germany, Greece, Italy, Portugal, Russia, Sweden, Switzerland and the United Kingdom) but also from other regions (Brazil, China, Japan, Kuwait, and the United States of America).

The decision to build SESAME was triggered by the gift from Germany of the $0.8 \mathrm{GeV}$ BESSY $1 \mathrm{I}$ storage ring and its injector, which consist of a $22 \mathrm{MeV}$ microtron (figure 1) and $0.8 \mathrm{GeV}$ booster synchrotron. A new $2.5 \mathrm{GeV}$ storage ring with a circumference of $133 \mathrm{~m}$ has been designed by SESAME staff in order to meet the users' demands. This compact ring provides twelve straight sections for wiggler 2 and undulator 3 insertion devices, which will produce very intense synchrotron light over a broad band of wavelengths, a feature which puts SESAME

\footnotetext{
${ }^{1}$ BESSY stands for Berlin electron storage society ring for synchrotron radiation.

${ }^{2}$ Wiggler is a periodic magnet array for the emission of very intense synchrotron light over a broad band of wavelengths Brown et al., 1983.

${ }^{3}$ Undulator is a periodic array of magnets for the emission of concentrated beams of synchrotron light with very high brilliance at well-defined wavelengths Brown et al., 1983.
} 
in the class of modern third generation intermediate energy storage rings. Table 1 summarizes the general storage ring parameters of the SESAME facility [Sesame team, 2012].

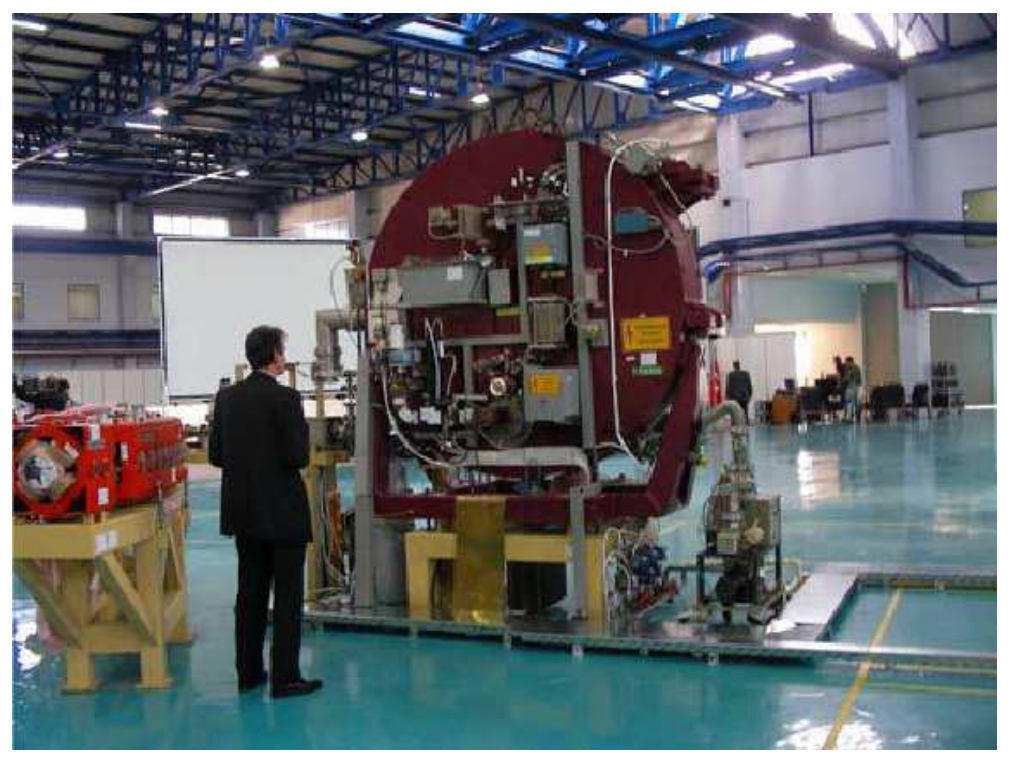

Figure 1: Photograph of the microtron in the SESAME experimental hall [Sesame team, 2013].

The SESAME users will be mostly from universities and research institutes in the Middle East. They will visit the laboratory periodically to carry out experiments, often in collaboration with scientists from other countries. SESAME will have well-equipped beamlines, experimental end stations, laboratories, and other support facilities will be available to users. Several hundred scientists, working in disciplines ranging from the biological and medical sciences to archaeology, are expected to use SESAME from day-one. This will make SESAME a unique multidisciplinary center in the region. As more beamlines are built, the number of users is expected to grow to about 1000 [Sesame team, 2012].

The main parameters that characterize the quality of synchrotron light sources are flux i.e., the amount of light delivered to an experimental sample, and brilliance i.e., a measure of the concentration of photons. SESAME bending magnets and multipole wigglers will provide a very high photon flux over the wide spectral range required for most applications. SESAME is expected to begin operations at the beginning of 2016. Seven beamlines are expected in Phase I, with three available at the start-up of the new ring. In the longer-term, up to 18 further beamlines can be added. Figure 2 shows schematically the layout of the SESAME machine and its beamlines [Sesame team, 2013]. 
Table 1: SESAME storage ring parameters Sesame team, 2013].

\begin{tabular}{|l|l|l|}
\hline Parameter & Unit & Value \\
\hline Energy & $\mathrm{GeV}$ & 2.5 \\
\hline Maximum beam current (200 bunches) & $\mathrm{mA}$ & 400 \\
\hline Circumference & $\mathrm{m}$ & 133.2 \\
\hline Bending dipole field & $\mathrm{T}$ & 1.45545 \\
\hline Energy loss (bending)/turn & $\mathrm{keV}$ & 590.2 \\
\hline RF (radiofrequency) & $\mathrm{MHz}$ & 499.654097 \\
\hline Harmonic number & & 222 \\
\hline RF power & $\mathrm{kW}$ & 500 \\
\hline Number of accelerating cavities & & 4 \\
\hline Total cavity voltage & $\mathrm{kV}$ & 2400 \\
\hline Natural bunch length & $\mathrm{mm}$ & 11.42 \\
\hline Beam Lifetime (@ P $=1 \mathrm{nTorr})$ & $\mathrm{hour}$ & 21.5 \\
\hline
\end{tabular}

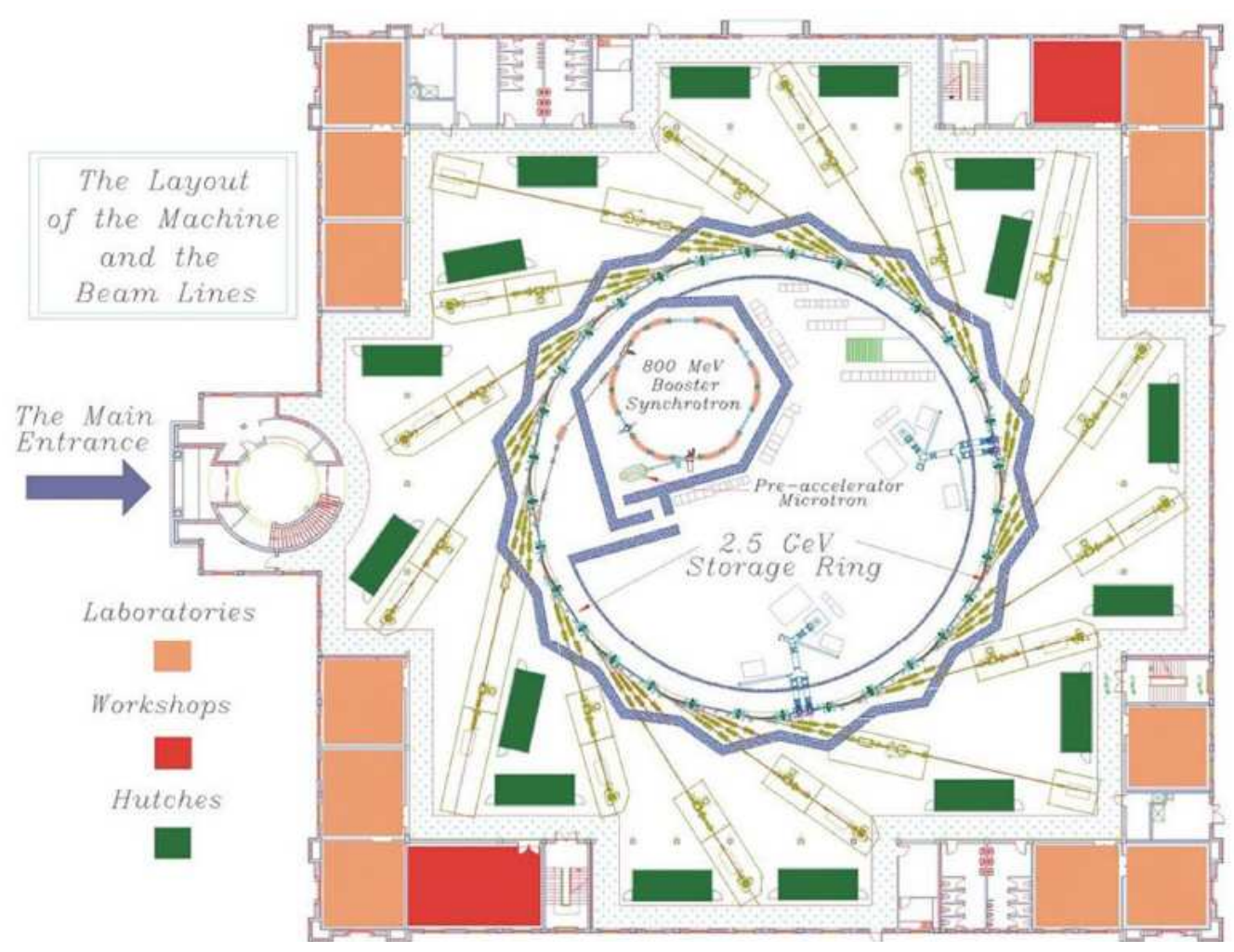

Figure 2: Scheme of SESAME machine and the beamlines Sesame team, 2013]. 
At SESAME the electrons will be injected from a $22 \mathrm{MeV}$ microtron, and accelerated to the maximum of $800 \mathrm{MeV}$ in a booster synchrotron. After that, electron bunches will be transported through a transfer line to a storage ring, where they will be accelerated to $2.5 \mathrm{GeV}$. Through the path from the microtron to and within the storage ring both destructive and non-destructive beam monitoring instruments will be installed. For instance, Faraday cups, fluorescent screens, current transformers, beam loss monitors, synchrotron light monitors, and beam position monitor pick-ups [Varnasseri, 2005].

The Portuguese "Fundação para a Ciência e a Tecnologia" (FCT), Lisbon, under an agreement with SESAME, offered four PhD fellowships for scientists and/or engineers from Members of SESAME to allow them to receive training and enhance their professional expertise in the fields of accelerator technologies, beamline instrumentation, and related applications, mainly in the following areas: (1) instrumentation and electronics; (2) software and computing specific to beamlines; and (3) vacuum and control systems.

After completion of the training period, the successful trainees may be offered a working position at the SESAME site in Jordan, depending on the results of their work carried out whilst in receipt of a fellowship and the availability of positions at the Center.

Within the aforementioned agreement, the University of Coimbra and LIP have made a selection of candidates to work within the ICNAS project described in this $\mathrm{PhD}$ thesis, which aims at using one beamline of ICNAS cyclotron (proton PET cyclotron), to instrument a self-developed system capable of (1) monitoring beam variables, and (2) controlling the dose on target based on electronics and instruments coupled to a self-developed computer application located outside the bulky cyclotron bunker. 


\section{Chapter 1}

\section{Physics and biology of proton therapy}

\subsection{Introduction}

According to estimates by the wold health organization (WHO), the number of new cancer patients in 2012 is about 14 million worldwide, which is expected to increase to 22 million by the year 2030. This represents an increase of $75 \%$ compared with 2008. As a result. cancer will then be the main cause of death [Cancer Research UK, 2012; Ferlay et al., 2010, 2014]. Cancer is considered to be responsible for $25 \%$ of all deaths in Europe. Moreover, it is the largest killer of people aged between 45 - 64 in Europe [Niederlaender, 2006]. World cancer statistics for the most common cancers (excluding non-melanoma skin cancer) in 2012 can be found in table 1.1. It can be noticed that lung cancer is the most common cancer worldwide contributing $13 \%$ of the total number of new cases diagnosed for both sexes.

Radiotherapy, surgery, and chemotherapy are the main therapies against cancer. Often various treatment possibilities are combined. The choice of treatment depends on cancer type (table 1.1), location, stage of the disease, and the general state of the patient. At present, in industrialized countries, about $70 \%$ of cancer patients are referred to a radiation therapy department for at least part of the treatment [IAEA and ICRU, 2008]. At the time of diagnostics, about $58 \%$ of tumors have not formed metastasis (figure 1.1). Radiation therapy uses high-energy 
Table 1.1: World cancer statistics for the most common cancers (excluding nonmelanoma skin cancer) in 2012 for both sexes [Ferlay et al., 2014].

\begin{tabular}{ccc}
\hline Cancer & $\begin{array}{c}\text { New cases diagnosed } \\
\text { in 2012 (1,000s) }\end{array}$ & Percent of all cancers* \\
\hline Lung & 1825 & 13.0 \\
Breast & 1677 & 11.9 \\
Colorectum & 1361 & 9.7 \\
Prostate & 1112 & 7.9 \\
Stomach & 952 & 6.8 \\
Liver & 782 & 5.6 \\
Cervix uteri & 528 & 3.7 \\
Oesophagus & 456 & 3.2 \\
Bladder & 430 & 3.1 \\
Non-Hodgkin lymphoma & 386 & 2.7 \\
Leukaemia & 352 & 2.5 \\
Pancreas & 338 & 2.4 \\
Kidney & 338 & 2.4 \\
Corpus uteri (endometrium) & 320 & 2.3 \\
Lip, oral cavity & 300 & 2.1 \\
Thyroid & 298 & 2.1 \\
Brain, nervous system & 256 & 1.8 \\
Ovary & 239 & 1.7 \\
Melanoma of skin & 232 & 1.6 \\
Gallbladder & 178 & 1.3 \\
Larynx & 157 & 1.1 \\
Other pharynx & 142 & 1.0 \\
Multiple myeloma & 114 & 0.8 \\
Nasopharynx & 87 & 0.6 \\
Hodgkin lymphoma & 66 & 0.5 \\
Testis & 55 & 0.4 \\
Kaposi sarcoma & 44 &
\end{tabular}

* excluding non-melanoma skin cancer.

radiation to kill cancer cells by damaging their DNA (deoxyribonucleic acid 1).

Surgery and radiotherapy are today of crucial importance, being successful in $22 \%$ and $12 \%$ of cancer cases, respectively. Another $6 \%$ of the cases receive a

${ }^{1}$ DNA is the hereditary material in humans and almost all other organisms. 
combination of surgery and radiotherapy. About $55 \%$ of all cancer patients could be cured successfully if treatment modalities would be improved, such as the application of protons in radiation therapy Durante and Loeffler, 2010; Haberer, 2002; [AEA and ICRU, 2008].

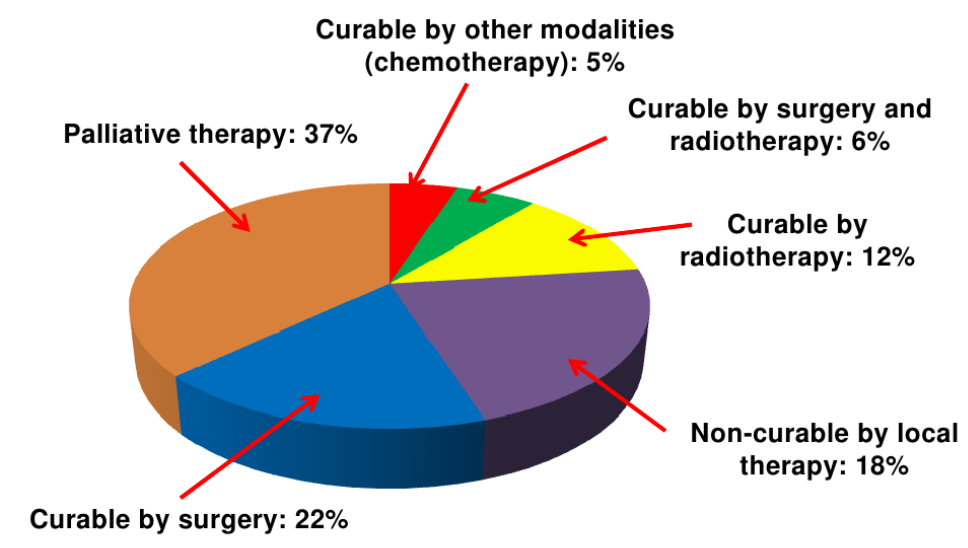

Localized cancer: $58 \%$

Metastatic cancer: $42 \%$

Figure 1.1: Present situations of the different treatment methods against cancer [GSI Nachrichten, 1999; Kraft, 2003].

Radiotherapy has greatly improved since its early days, by the time of the discovery of X-rays in 1895, and it is still in progress nowadays. Radiotherapy is not limited to external beam radiation, comprised traditionally by photons and electrons. There are other types of radiation treatments, such as internal radiation therapy i.e., brachytherapy 1 , charged particle therapy, and neutron therapy.

The use of ion beams in tumor treatment was first proposed more than 60 years ago, when the depth-dose characteristics of proton beams were investigated by Wilson [1946]. Since then, ion beam therapy has evolved and significantly expanded. A brief historical diagram of the emergence of particle therapy over the last century is presented in figure 1.2. The use of proton therapy as an alternative to conventional radiation has led to important developments and refinements in conventional treatment techniques. Protons and carbon ions are characterized by a property not shared by photons and electrons, the Bragg peak 2, which allows for highly conformal treatment of deep-seated tumors with great accuracy,

\footnotetext{
${ }^{1}$ Brachytherapy consists in applying radioactive seeds directly in contact with the tumor, while external therapy is a non-invasive way of delivering high energy particle beams to the tumor.

${ }^{2}$ The Bragg peak, named after William Bragg, who discovered it in 1905. He measured an increase of ionization at the end of the range of alpha particles in air.
} 

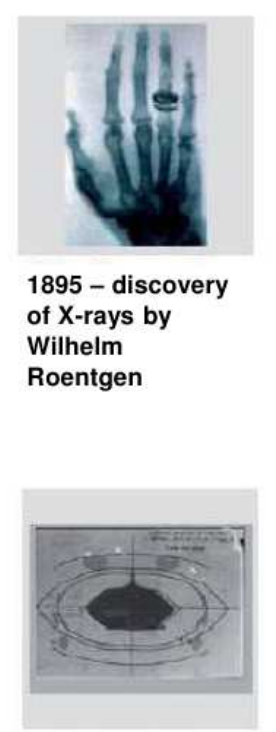

1961 -

collaboration

between Harvard

Cyclotron

Laboratory and

Massachusetts

General Hospital

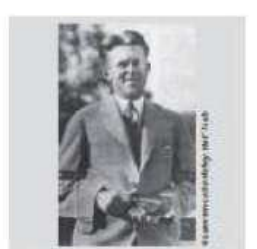

1932 - first cyclotron developed by Ernest Lawrence

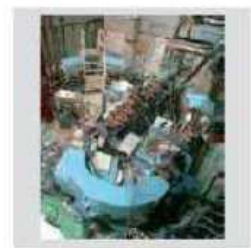

1993 - patients treated at the first hospital based facility at Loma Lina

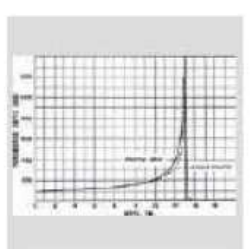

1946 - proton therapy proposed by Wilson, exploiting the properties of the Bragg peak

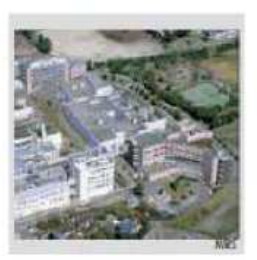

1994 - first facility ions operational at ions at GSI HIMAC, Japan

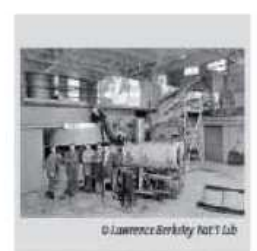

1954 - Berkeley treats the first patient and begins extensive studies with various ions
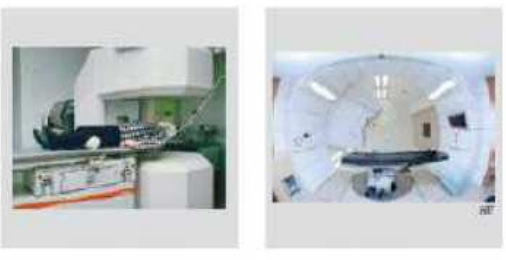

2009 - first

European proton carbon ion facility starts treatment in Heidelberg

Figure 1.2: Particle therapy evolution since the discovery of the X-ray by Roentgen in 1895 [ENLIGHT, 2013].

while delivering minimal doses to normal surrounding tissue. This characteristic may allow improving cancer treatment results, consequently contributing to the reduction of the mortality 1 rates due to cancer.

Hadrontherapy is an advanced technique of external radiation therapy, using either low-LET 2 ions such as protons or high-LET particles such as carbon ions. High-LET particles have additional advantages in respect to low-LET radiation, since the hight-LET particles produce more damages in the tumor located in the Bragg peak, allowing to treat tumors that are radioresistant to low-LET particles [IAEA and ICRU, 2008]. Patient statistics 3 of proton therapy facilities over all the world are listed in tables 1.2 and 1.3, whereas the patient statistics of carbon therapy facilities are listed in table 1.4. These statistics indicate a total of 37 medical facilities offering proton therapy and 7 facilities offering carbon ion

\footnotetext{
${ }^{1}$ Mortality is the number of deaths occurring in a given period in a specified population. Often given as an absolute number of deaths per vear or as a standardized rate per 100.000 [Cancer Research UK, 2012].

${ }^{2}$ LET stands for linear energy transfer and is described in section 1.2.1.3.

${ }^{3}$ Patient statistics of particle therapy facilities worldwide represents the number of patients treated in operation and in closed facilities until August 2013.
} 
therapy worldwide. Since the early 50's, more than 78,000 patients have been treated with protons and about 10,000 patients with carbon ions.

Table 1.2: Proton therapy facilities in operation in 2013 (A) [PTCOG, 2013].

\begin{tabular}{|c|c|c|c|c|}
\hline $\begin{array}{l}\text { Who, } \\
\text { Where }\end{array}$ & Country & $\begin{array}{l}\text { S/C* Max. } \\
\text { energy }(\mathrm{MeV})\end{array}$ & $\begin{array}{l}\text { Start of } \\
\text { treatment }\end{array}$ & $\begin{array}{l}\text { Total } \\
\text { patients } \\
\text { treated }\end{array}$ \\
\hline ITEP, Moscow & Russia & S 250 & 1969 & 4246 \\
\hline St.Petersburg & Russia & S 1000 & 1975 & 1386 \\
\hline PSI, Villigen & Switzerland & C 250 & 1996 & 1409 \\
\hline Dubna & Russia & $200 * *$ & 1999 & 922 \\
\hline Uppsala & Sweden & C 200 & 1989 & 1267 \\
\hline Clatterbridge & England & C 62 & 1989 & 2297 \\
\hline Loma Linda & CA.,USA & S 250 & 1990 & 16884 \\
\hline Nice & France & $\mathrm{C} 65$ & 1991 & 4692 \\
\hline Orsay & France & C 230 & 1991 & 5949 \\
\hline NRF - iThemba Labs & South Africa & C 200 & 1993 & 521 \\
\hline $\begin{array}{l}\text { IU Health PTC, } \\
\text { Bloomington }\end{array}$ & IN.,USA & C 200 & 2004 & 1688 \\
\hline UCSF & CA.,USA & C 60 & 1994 & 1515 \\
\hline TRIUMF, Vancouver & Canada & C 72 & 1995 & 170 \\
\hline HZB (HMI), Berlin & Germany & C 72 & 1998 & 2084 \\
\hline NCC, Kashiwa & Japan & C 235 & 1998 & 1226 \\
\hline HIBMC,Hyogo & Japan & S 230 & 2001 & 3198 \\
\hline $\operatorname{PMRC}(2)$, Tsukuba & Japan & S 250 & 2001 & 2516 \\
\hline NPTC, MGH Boston & MA.,USA & C 235 & 2001 & 6550 \\
\hline INFN-LNS, Catania & Italy & C 60 & 2002 & 293 \\
\hline SCC, Shizuoka & Japan & S 235 & 2003 & 1365 \\
\hline $\begin{array}{l}\text { Cancer Center } \\
\text { STPTC, Koriyama-City }\end{array}$ & Japan & S 235 & 2008 & 1812 \\
\hline WPTC, Zibo & China & C 230 & 2004 & 1078 \\
\hline $\begin{array}{l}\text { MD Anderson Cancer } \\
\text { Center. Houston }\end{array}$ & TX.,USA & S 250 & 2006 & 3909 \\
\hline UFPTI, Jacksonville & FL.,USA & C 230 & 2006 & 4272 \\
\hline NCC, IIsan & South Korea & C 230 & 2007 & 1041 \\
\hline RPTC, Munich & Germany & C 250 & 2009 & 1377 \\
\hline $\begin{array}{l}\text { ProCure PTC, } \\
\text { Oklahoma City }\end{array}$ & OK.,USA & C 230 & 2009 & 1045 \\
\hline
\end{tabular}


Table 1.3: Proton therapy facilities in operation in 2013 (B) [PTCOG, 2013].

\begin{tabular}{|c|c|c|c|c|}
\hline $\begin{array}{l}\text { Who, } \\
\text { Where }\end{array}$ & Country & $\begin{array}{l}\text { S/C* Max. } \\
\text { energy }(\mathrm{MeV})\end{array}$ & $\begin{array}{l}\text { Start of } \\
\text { treatment }\end{array}$ & $\begin{array}{l}\text { Total } \\
\text { patients } \\
\text { treated }\end{array}$ \\
\hline HIT, Heidelberg & Germany & S 250 & 2009 & 252 \\
\hline UPenn, Philadelphia & PA.,USA & C 230 & 2010 & 1100 \\
\hline $\begin{array}{l}\text { CDH Proton Center, } \\
\text { Warrenville }\end{array}$ & IL.,USA & C 230 & 2010 & 840 \\
\hline HUPTI, Hampton & VA., USA & C 230 & 2010 & 489 \\
\hline IFJ PAN, Krakow & Poland & C 60 & 2011 & 15 \\
\hline Medipolis PTRC, Ibusuki & Japan & S 250 & 2011 & 490 \\
\hline $\begin{array}{l}\text { CNAO, Pavia } \\
\text { ProCure Proton }\end{array}$ & Italy & S 250 & 2011 & 58 \\
\hline $\begin{array}{l}\text { Therapy Center, } \\
\text { Somerset }\end{array}$ & NJ., USA & C 230 & 2012 & 137 \\
\hline $\begin{array}{l}\text { PTC Czech r.s.o., Prague } \\
\text { SCCA, Proton Therapy, }\end{array}$ & Czech Republic & C 230 & 2012 & 1 \\
\hline $\begin{array}{l}\text { a ProCure Center, } \\
\text { Seattle }\end{array}$ & WA., USA & C 230 & 2013 & 1 \\
\hline
\end{tabular}

Table 1.4: Carbon therapy facilities in operation in 2013 [PTCOG, 2013].

\begin{tabular}{lllll}
\hline $\begin{array}{l}\text { Who, } \\
\text { Where }\end{array}$ & Country & $\begin{array}{l}\text { S/C* Max. } \\
\text { energy }(\mathbf{M e V})\end{array}$ & $\begin{array}{l}\text { Start of } \\
\text { treatment }\end{array}$ & $\begin{array}{l}\text { Total } \\
\text { patients } \\
\text { treated }\end{array}$ \\
\hline HIMAC, Chiba & Japan & $\mathrm{S} 800 / \mathrm{u}$ & 1994 & 7331 \\
HIBMC,Hyogo & Japan & $\mathrm{S} 320 / \mathrm{u}$ & 2002 & 788 \\
HIT, Heidelberg & Germany & $\mathrm{S} 430 / \mathrm{u}$ & 2009 & 980 \\
GHMC, Gunma & Japan & $\mathrm{S} 400 / \mathrm{u}$ & 2010 & 537 \\
IMP-CAS, Lanzhou & China & $\mathrm{S} 400 / \mathrm{u}$ & 2006 & 194 \\
CNAO, Pavia & Italy & $\mathrm{S} 400 / \mathrm{u}$ & 2012 & 22 \\
SAGA-HIMAT, & Japan & $\mathrm{S} 400 / \mathrm{u}$ & 2013 & 2 \\
Tosu & \multicolumn{4}{l}{}
\end{tabular}




\subsection{Rationale for proton therapy}

Proton therapy is a unique field at the border between physics, biology, and medicine. The potential advantages of using protons in radiotherapy over conventional radiotherapy with photons or electrons arise from these three science domains.

\subsubsection{Physical rationale for proton therapy}

The precise range of charged particles in matter is the main advantage offered by hadrons compared to conventional radiotherapy treatments. A comparison of depth-dose curves for photon, proton, and carbon ion beams is shown in figure 1.3 . It can be seen that the dose decreases exponentially with penetrating depth for conventional radiation (photons). However, charged particle beams lose a small quantity of its energy at the access until the maximum penetration depth is reached. There, the remaining energy is lost over a short distance, resulting in the high dose peak, the so-called Bragg peak.

In radiotherapy, the challenge is to destroy cancer cells without affecting the surrounding healthy tissue. Contrary to X-rays and electrons, protons and heavier ions e.g., carbon ions, deposit a large part of their energy at a specific point near the end of their path instead of all along their path. This means that one can bring large amounts of energy exactly where needed without causing significant damage along the way.

Normally, the target volume of tumors is much larger than the unmodified Bragg peak width, as shown in figure 1.4, left. In the treatment of large tumors, the Bragg peak must be broadened using overlapping beams with different energies. One technique is accomplished through modulating the particle energy from pulse to pulse. Here, several pristine Bragg peaks form a superposition of Bragg peaks (figure 1.4, right). A relatively consistent dose distribution can also be delivered to tumors by spreading out the Bragg peak through range and intensity modulation, which can be achieved by using a physical device e.g., ridge filter or modulation wheel. The resulting spread-out Bragg peak (SOBP) aims to provide a constant biologically effective dose within the tumor. The physical dose profile is corrected according to the RBE 1 in order to provide a flat SOBP in the tumor region. For protons, a constant RBE of 1.1 is used to convert the physical dose

\footnotetext{
${ }^{1} \mathrm{RBE}$ stands for relative biological effectiveness and is described in section 1.2.2.2
} 


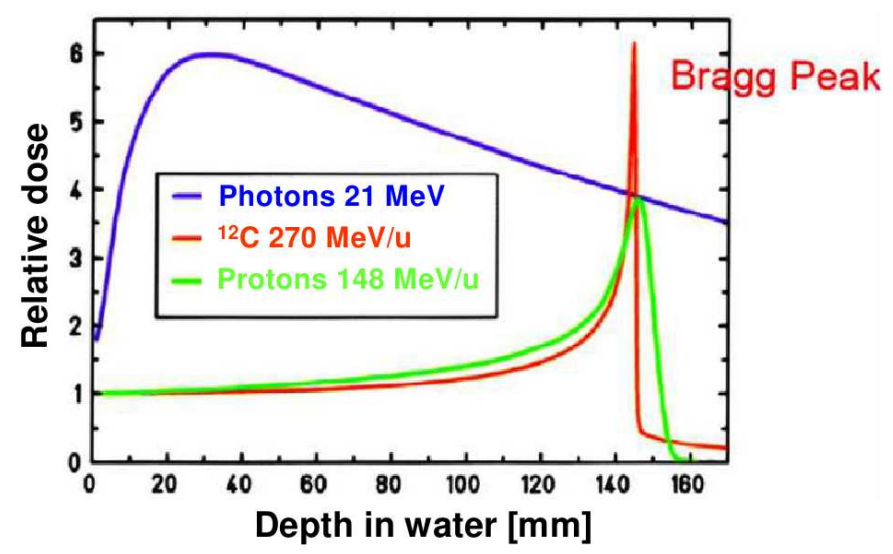

Figure 1.3: Depth-dose profiles for photons, protons, and carbon ions. It can be seen that a large part of the energy of protons and carbon ions is liberated in a very narrow peak known as the Bragg peak Crespo, 2005; Fokas et al., 2009; Kraft, 2000].

to the biological effective dose, whereas for carbon ions the RBE increases with increasing depth in the tissue [Kraft, 2000].
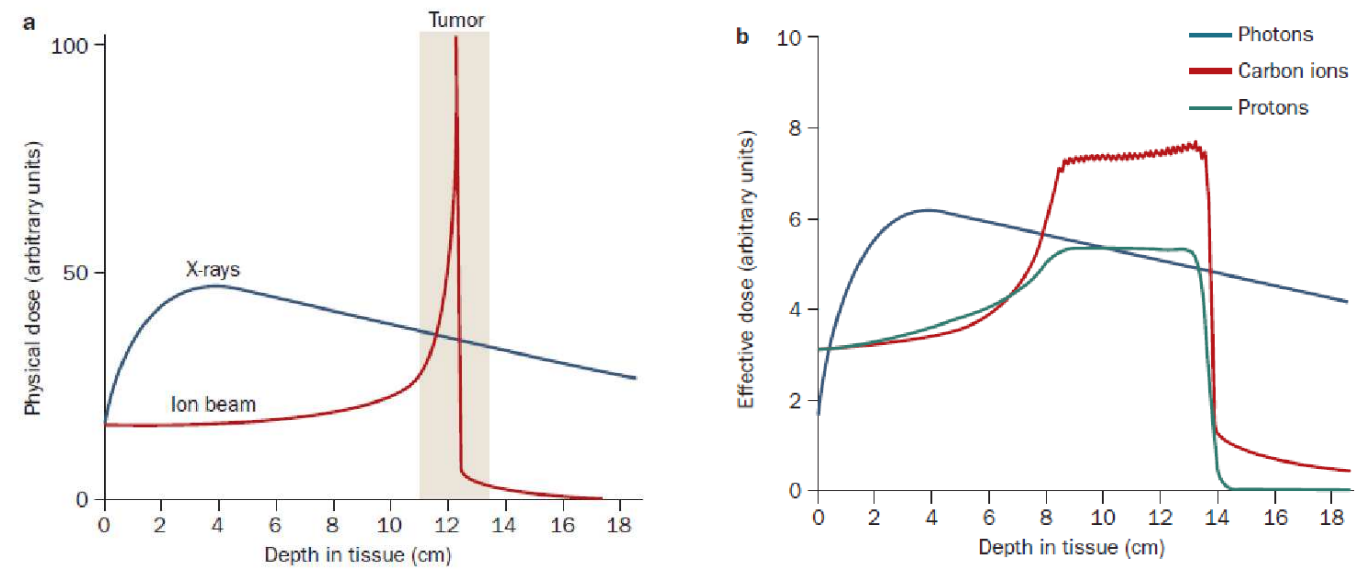

Figure 1.4: Charged particles have an advantageous radiation depth-dose profile compared with photons. Left: a comparison of the depth-dose profiles for photons and charged particles. Right: superposition of Bragg peaks produced from several pristine Bragg peaks [Durante and Loeffler, 2010]. 


\subsubsection{Stopping power and the Bethe-Bloch formula}

The main advantage of charged particles over photons is the fact that charged particles stop within matter at a known depth, with a maximum energy deposition in the Bragg peak. This effect is characterized by the stopping power, which is defined as the average energy loss per unit path length, resulting mainly from Coulomb interactions with bound atomic electrons of the medium. In addition, charged particles also undergo elastic interactions with atomic nuclei of the medium [Leo, 1994]. The electronic stopping power has a prevalent contribution to the total stopping power, whereas the nuclear stopping power has a minor effect. The contribution of elastic nuclear collisions to total energy loss is very little (less than 0.1\%), when compared with the contribution from electronic stopping power [Janni, 1982].

Since the energy dissipated in each electronic collision is very small, the energy loss of the incident charged particle is practically continuous. The mean energy loss is described by the Bethe-Bloch formula (equation 1.1). It is also called stopping power and (unrestricted) linear energy transfer 1 .

$$
-\frac{d E}{d x}=K z_{e f f}^{2} \frac{Z}{A} \frac{1}{\beta^{2}}\left[\frac{1}{2} \ln \frac{2 m_{e} c^{2} \beta^{2} \gamma^{2} T_{\max }}{I^{2}}-\beta^{2}-\frac{\delta}{2}\right]
$$

where $T_{\text {max }}$ is the maximum kinetic energy that can be imparted to a free electron in a single collision (equation 1.2). Other variables are defined in table 1.5.

$$
T_{\max }=\frac{2 m_{e} c^{2}\left(\gamma^{2}-1\right)}{1+2 \gamma \frac{m_{e}}{M}+\left(\frac{m_{e}}{M}\right)^{2}}
$$

where $M$ is the ion mass (at rest) and $\gamma$ is the Lorentz factor. Moreover, if $M \ggg m_{e}$, then $T_{\max } \simeq 2 m_{e} c^{2}\left(\gamma^{2}-1\right)$ [Leo, 1994].

The two key factors to understand the appearance of the Bragg peak of protons near the end of their range are $\frac{1}{\beta^{2}}$ and $z_{\text {eff }}$. The $\frac{1}{\beta^{2}} \simeq \frac{1}{E}$ factor yields an increasing energy loss with decreasing particle energy (equation 1.1), which can be seen in figure 1.5 for several particles, with the stopping power displayed with units of LET i.e, $\mathrm{keV} / \mu \mathrm{m}$.

The relation between the velocity of a projectile $\beta$ and $z_{\text {eff }}$ is given by the

\footnotetext{
${ }^{1}$ The essential difference between the stopping power and the linear energy transfer is explained in section 1.2.1.3
} 
Table 1.5: Variables and values of the Bethe-Bloch equation [Leo, 1994].

\begin{tabular}{lll}
\hline Symbol & Definition & Unit or Value \\
\hline$\frac{d E}{d x}$ & Energy loss & $\mathrm{MeV} \mathrm{g}^{-1} \mathrm{~cm}^{2}$ \\
$A$ & Atomic number & $g \mathrm{~mol}^{-1}$ \\
$\frac{K}{A}$ & $4 \pi N_{A} r_{e}^{2} c^{2} / A$ & $0.307075 \mathrm{MeV} \mathrm{g}^{-1} \mathrm{~cm}^{2}$ \\
$N_{A}$ & Avogadro's number & $6.0221367(36) \times 10^{23} \mathrm{~mol}^{-1}$ \\
$Z_{e f f}$ & Effective charge of incident particle & \\
$Z$ & Atomic number of medium & \\
$m_{e} c^{2}$ & Electron rest energy & $0.51099906(15) \mathrm{MeV}$ \\
$I$ & Mean excitation energy & $\mathrm{eV}$ \\
$\delta$ & Density effect, correction to & \\
$\beta$ & ionization energy loss & \\
$\gamma$ & $v / c$ of the incident particle & \\
\hline
\end{tabular}

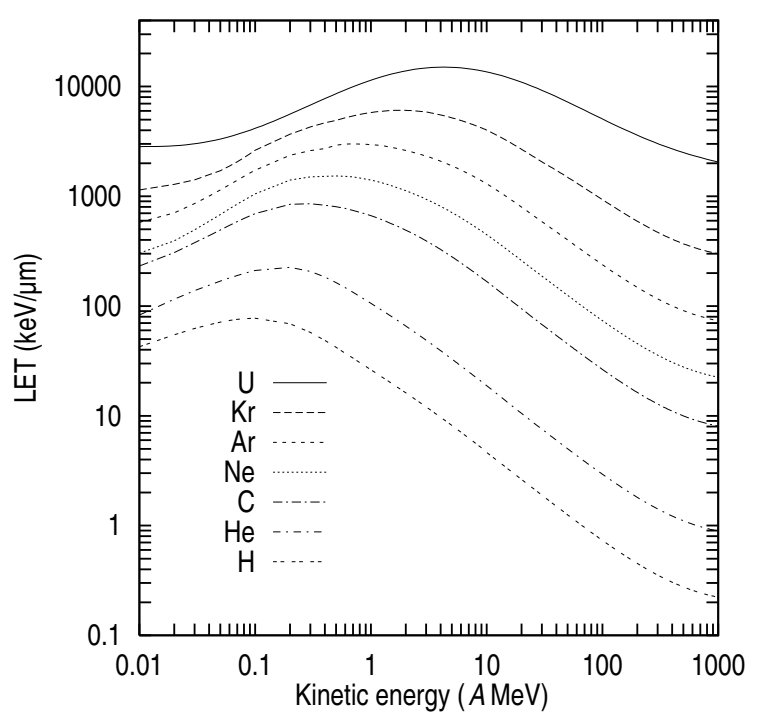

Figure 1.5: Energy loss in water for several ions. The curves were calculated with the computer code ATIMA (atomic interactions with matter) [Crespo, 2005; Schwab, 1991

Barkas formula (equation 1.3) Barkas et al., 1956].

$$
z_{e f f}=z\left(1-e^{-125 \beta z^{2 / 3}}\right)
$$

For heavier ions at low energies a positive projectile starts collecting electrons from the surrounding target material, thus rapidly decreasing its effective charge 
$z_{\text {eff }}$. Therefore, the two contributions $\left(\frac{1}{\beta^{2}}\right.$ and $\left.z_{\text {eff }}\right)$ cause the special maximum of energy loss i.e., the Bragg peak. The low energy loss at higher energies explains the quasi constant plateau of energy deposition at the entrance of the target [Crespo, 2005; Kraft, 2000].

\subsubsection{Absorbed dose}

The primary physical quantity in dosimetry is the dose $D$, defined as the mean energy $d E$ deposited by ionizing radiation in a mass element $d m$ (equation 1.4) [CRU, 1970].

$$
D=d E / d m
$$

Dose is measured in Gray $(G y)$, where $1 G y=1 \mathrm{~J} \mathrm{~kg}^{-1}$.

For a parallel beam of particles with a fluence $F$, the dose deposited in a thin slice of an absorber with mass density $\rho$ can be calculated with the formula reported in equation 1.5 [Ahmed, 2007; Crespo, 2005; Kraft, 2000].

$$
D[G y]=1.6 \times 10^{-9} \cdot \frac{d E}{d x}\left[\frac{k e V}{\mu m}\right] \cdot F\left[\frac{1}{c m^{2}}\right] \cdot \frac{1}{\rho}\left[\frac{\mathrm{cm}^{3}}{g}\right]
$$

where $\frac{d E}{d x}$ represents the total energy loss 11 of ions in the irradiated material.

\subsubsection{Linear energy transfer (LET)}

When ionizing radiation traverses through matter, it loses energy progressively through various interaction processes along the length of its path. For a particular material, the rate of loss of energy depends on energy and type of radiation as well as the density of the material.

The concept of linear energy transfer (LET) was introduced by Zirkle et al. [1952] to describe the quality of radiation. It represents the rate at which energy is deposited by the ionizing particles. In such terms, LET and stopping power represent the same quantity. Nevertheless, the essential difference between the stopping power and the LET resides in the fact that some secondary electrons created by the projectile have enough energy to travel away from the interaction

\footnotetext{
${ }^{1}$ The total energy loss, defined as the sum of electronic and nuclear stopping powers.
} 
Table 1.6: Typical LET values of ionizing radiation Hall and Giaccia, 2006].

\begin{tabular}{ll}
\hline Radiation & LET $(\mathrm{keV} / \mu \mathrm{m})$ \\
\hline$C o-60 \gamma$-rays & 0.2 \\
$250 k V_{p}$ X-rays & 2.0 \\
$10 \mathrm{MeV}$ protons & 4.7 \\
$150 \mathrm{MeV}$ protons & 0.5 \\
$14 \mathrm{MeV}$ neutrons & 12 \\
$2.5 \mathrm{MeV} \alpha$ particles & 166 \\
$2 \mathrm{GeV}$ Fe ions & 1000 \\
\hline
\end{tabular}

point and to deposit their energy in a different area. Consequently, their contribution to the main particle energy loss is then only included in the LET if their energy is lower than the subscript of the LET [Turner, 2007].

The LET is often expressed in $M e V / m m$ (or $k e V / \mu m$ ). In ICRU'16, the LET is defined as reported in equation 1.6 [CRU, 1970].

$$
L_{\Delta}=\left(\frac{d E}{d l}\right)_{\Delta}
$$

where $L_{\Delta}$ is the LET, $d l$ is the distance traversed by the particle, and $d E$ is the mean energy loss due to those collisions which have an energy transfer smaller than a specified energy cut-off value $\Delta$. For instance, $L_{\infty}$ designates the LET when all possible energy transfers are included and in this case the concept of nonrestricted stopping power is used, whereas $L_{100}$ means a cut-off value of $100 \mathrm{eV}$ and it is referred to as restricted stopping power. In such case, the secondary electrons with energy more than $100 \mathrm{eV}$ are not taken into account, as they leave the volume of interest around the primary particle track [CRU, 1970].

In general, ionizing radiation is categorized into low-LET and high-LET, with charged particle radiation usually being high-LET, because of their greater energy deposition along their paths, whereas X-rays are categorized as low-LET because of their sparse ionizations. When a charged particle deposits energy in a target, it slows down. Consequently, the LET varies along the path of the charged particle, whereas the energy loss i.e., $d E / d x$ increases (the Bragg peak). Typical LET values for various types of radiation are listed in table 1.6. 


\subsubsection{Multiple Coulomb scattering and range straggling}

Proton scattering is mainly a result of deflections by atomic nuclei, which is referred to as multiple Coulomb scattering (MCS). Although the strong interactions also contributes to this phenomenon, most deflections are however due to the elastic scattering with target nuclei via the Coulomb force [Janni, 1982]. Indeed, as the proton mass $\left(\sim 938 \mathrm{MeV} / \mathrm{c}^{2}\right)$ is about $2 \times 10^{3}$ times higher than that of the electron $\left(\sim 511 \mathrm{keV} / \mathrm{c}^{2}\right)$, electrons hardly deflect protons. The mass ratio also explains why heavier ions like carbon ions are deflected much less than protons [Gottschalk, 2004].

The range of a monoenergetic beam of charged particles with energy $E$ is defined as the penetration depth at which half of its initial particles have already stopped [Crespo, 2005]. The reciprocal of the stopping power gives the distance traveled per unit energy loss. Therefore, the range $R$ is the integral of the stopping power down to zero energy (equation 1.7) [Turner, 2007].

$$
R=\int_{0}^{T}-\frac{1}{\mathrm{dE} / \mathrm{dx}} \mathrm{d} E
$$

where $T$ is the particle kinetic energy.

Since the energy loss against atomic electrons is a stochastic process, a spread in energy happens after a monoenergetic beam has passed through a given target thickness. The energy spread leads to range straggling, which is defined as the fluctuation in path length for individual particles of the same initial energy. As a charged particle traverses a medium, statistical fluctuations occur in the number of collisions along its path and in the amount of energy lost in each collision. Consequently, identical particles starting out under the same conditions will show a distribution of energies as they pass a given depth and a distribution of path lengths traversed before they stop. The phenomenon of unequal energy losses under the same conditions is referred to as energy straggling. The phenomenon of unequal path lengths is called range straggling [Turner, 2007].

The main contribution to multiple Coulomb scattering theories was developed by Molière in 1948. The theory of Molière was found to be accurate to better than $1 \%$ on average for protons. It describes the shape of the particle angular distribution and its characteristic width. The Coulomb scattering distribution is roughly Gaussian for small deflection angles $\left(<10^{\circ}\right)$, however it deviates markedly from a Gaussian shape for larger angles [Gottschalk et al., 1993]. For small angles $\theta$, it is preferable to use a Gaussian approximation with a width given 
by [Highland, 1975; Kraft, 2000]:

$$
\sigma_{\theta}=\frac{14.1 \mathrm{MeV}}{\beta p c} z \sqrt{\frac{x}{X_{0}}\left(1+\frac{1}{9} \log _{10} \frac{x}{X_{0}}\right)},
$$

where $\sigma_{\theta}$ is the mean scattering angle, $p$ and $v$ are the particle momentum and speed, $z$ the projectile charge number. Finally, $x$ and $X_{0}$ are the target thickness and radiation length 1 , respectively.

A comparison of lateral spreading of photon, carbon, and proton beams in water is shown in figure 1.6. This figure reveals that carbon ions have the best lateral characteristics and protons have a worse lateral spread than well collimated photon beams, at depths larger than $10 \mathrm{~cm}$.

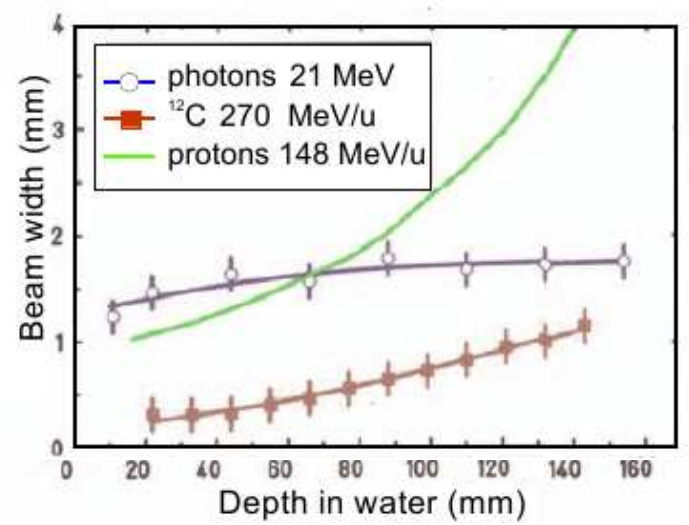

Figure 1.6: Beam width at different depths in water of photons, protons and carbon ions [Crespo, 2005; Kraft, 2000]. The corresponding relative depth-dose profiles are shown in figure 1.3. Despite the initial difference in beam widths, a lower lateral scattering among the charged species is verified for carbon ions.

\subsubsection{Radiobiological rationale for proton therapy}

Ionizing radiation was used in the treatment of cancer shortly after the discovery of X-ray radiation in 1895. At that time, the processes responsible for killing of cancer cells were poorly understood. However, it is now known that radiation causes damage to the DNA of living cells, and has a greater biological effect on

\footnotetext{
${ }^{1}$ Radiation length is the mean path length required to reduce the energy of relativistic charged particles by the factor $1 / \mathrm{e}$, or 0.368 , as they pass through matter Nakamura et al., 2010.
} 
cancer cells compared to normal tissue Jackson, 2002. DNA is the heart of the cell. It contains all the instructions for producing new cells. It is a complex molecule formed of two long strands that are twisted around each other and linked by chemical subunits. The DNA represents roughly $5 \%$ of the cell nucleus volume and its size is around $10 \mu \mathrm{m}$ in diameter Elsässer and Scholz. 2007: Shikazono et al., 2006].

\subsubsection{Biological effects of clustered DNA damage}

Ionizing radiation deposits energy in its path, mostly in the form of secondary electrons, which damage the DNA by creating molecular dissociation. In other words, DNA may be damaged due to ionization caused by radiation, as presented in figure 1.7. Clustered DNA damage would be produced where the density of ionization is high, whereas the isolated damage would be generated where the ionization density is low.

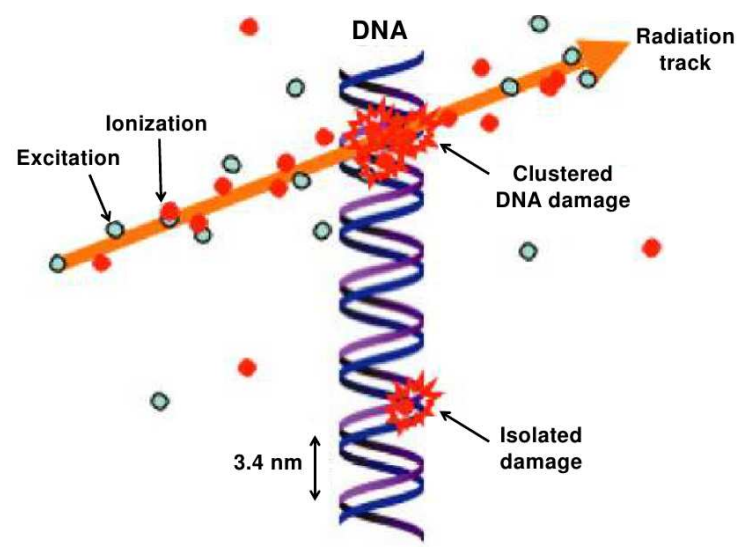

Figure 1.7: Schematic figure of radiation damage to DNA [Advanced Science Research, 2008]. There are two main ways where the radiation can damage DNA inside living cells. Radiation can strike the DNA molecule directly, ionizing and damaging it. Alternatively, radiation can ionize water molecules, producing free radicals that react with and damage DNA molecules.

The most severe damage to the cell results when the DNA is injured. There are two major actions that produce radiation injuries to the DNA inside cells: Direct and indirect actions. In the case of indirect action, the water inside cells tends to absorb a large portion of radiation (high frequency radiation or fast moving particles) and becomes ionized. After that, it produces free radicals 11 in the cell

\footnotetext{
${ }^{1}$ Free radicals are short lived and highly reactive molecules such as $O H \cdot$.
} 
environment. These free radicals such as $O H \cdot$ (hydroxyl radical) can react with and damage the DNA molecule. On the other hand, in direct action, radiation can interact with the DNA molecule through Coulomb interactions, ionizing and damaging it directly (figure 1.7). Photon irradiation yield mainly indirect effects, while the opposite is observed for charged particles [Forshier, 2002].

A known important factor that can enhance the radiation damage is the amount of oxygen in the cells. The presence or absence of molecular oxygen within a cell influences the biological effect of ionizing radiation: the larger the cell oxygenation above anoxia, the larger is the biological effect of ionizing radiation.

High-LET radiation is more effective at creating double strand breaks [Hada and Georgakilas, 2008], and this is one of the factors that creates demand for ion therapy. The desired outcome of a critical interaction is the death or halting of function of most cancer cells and the successful repair of healthy cells.

\subsubsection{Relative biological effectiveness (RBE)}

High-LET particles such as carbon ions have additional advantages over lowLET, since high-LET particles produce more damages in the tumor located in the Bragg peak and allow to treat tumors that are radioresistant to low-LET particles such as photons and protons [IAEA and ICRU, 2008].

The LET of a given particle species is closely correlated with its biological effectiveness, or the ability of the radiation to permanently damage cancer cells [Hada and Georgakilas, 2008]. The relative biological effectiveness (RBE) is defined in equation 1.9, as the ratio of X-rays and ion dose producing the same biological effect.

$$
R B E=\left.\frac{D_{X-\text { rays }}}{D_{\text {ion }}}\right|_{\text {isoeffect }}
$$

Moreover, different types of radiation have, for the same dose delivered to the target volume, a different effectiveness in killing tumor cells, as shown in figure 1.8

The RBE of protons is assumed to be 1.1, based on a broad range of biological end points, such as dose required to kill $90 \%$ of cells, over the full clinical range. 


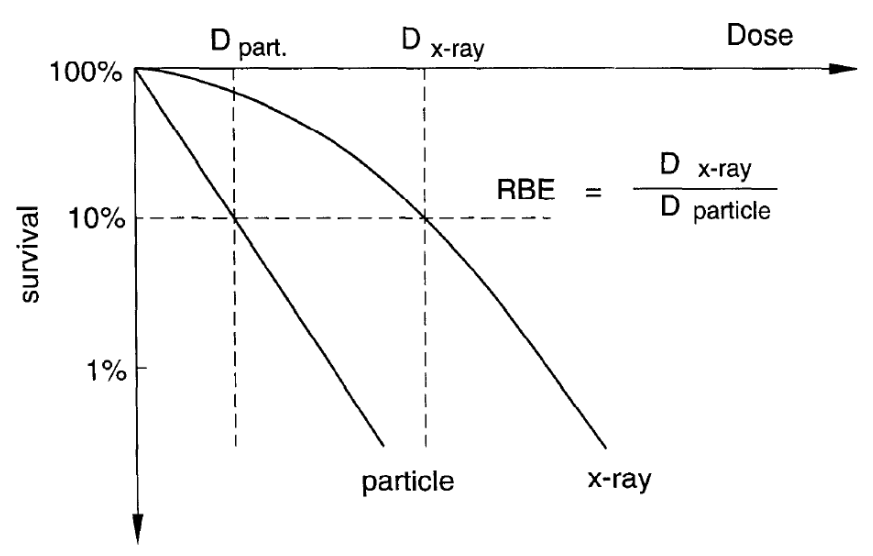

Figure 1.8: Definition of RBE, the relative biological effectiveness, illustrated with cell survival curves [Kraft, 2000].

This means that proton beams produce practically the same lethal damage as conventional photon beams. For carbon ions, the RBE can range between 2 and 5 and it increases before the stopping of the ion at the end of its range. The reason for the higher $\mathrm{RBE}$ is that the ionization density produced by a carbon ion traversing a cell is twenty times larger than the one of a proton having the same range [Kraft, 2007].

\subsection{Advantages in comparison to photons}

In radiotherapy, the main advantage of protons over photons is that protons stop within matter at a known depth, with a maximum energy deposition within the Bragg peak (figure 1.3). The precise range of protons in matter is the main advantage offered by protons compared to conventional X-ray radiation. The better ballistic of ions over photons allows decreasing the dose to surrounding tissues by a factor of $2-10$ [Dosanjh et al., 2010]. The main motivation behind the use of protons for cancer treatment is its inverted depth-dose profile, i.e, the Bragg peak, which is capable of greatly improving the dose profile over that of photon radiation. Unlike protons and heavier charged particles, the depthdose profile of photons shows the highest energy deposition shortly after their entrance in the tissue, which then decreases exponentially as the penetration depth increases (figure 1.3) Brown and Suit, 2004.

The initial energy of the protons can be adjusted in order to deposit the maximum dose at a specific depth in a patient, in contrast to photons which 
Table 1.7: General cost estimation of radiation therapy for combined proton/carbon, proton-only, and photon facilities [Peeters et al., 2010].

\begin{tabular}{llll}
\hline & Combined & Proton-only & Photon \\
\hline Capital costs (million EUR) & 138.6 & 94.9 & 23.4 \\
Total costs per year (million EUR) & 36.7 & 24.9 & 9.6 \\
Cost per fraction (EUR ) & 1128 & 743 & 233 \\
\hline
\end{tabular}

deliver dose through the entire patient. This gives an advantage to protons, where there is no dose deposited behind the tumor, thus reducing the probability of complications and late effects such as secondary tumor formation [Brown and Suit, 2004]. Moreover, photons lead to a larger volume of normal tissues exposed to low doses and to an increased total body exposure, which are both known to increase the risk of radiation-induced secondary cancer Blakely and Chang, 2004 .

Another advantage of protons is that the beam can be driven by magnetic deflection. Therefore, protons can be directed and shaped in a suitable way. The lateral scattering of protons is an advantage in comparison to photons, but only if the penetration depth does not exceed $7 \mathrm{~cm}$, because above this value, lateral scattering of protons increases very steeply (figure 1.6).

Particle therapy has potentially a better therapeutic rationale than photon therapy. But, investment costs are much higher. It is estimated that the cost ratio particle/photon therapy is about 4.8 for combined proton/carbon facility and 3.2 for proton-only facility [Peeters et al., 2010]. General costs for combined proton/carbon, proton-only, and photon facilities are presented in table 1.7.

\subsection{Advantages in comparison to other charged particles}

Protons have common features with heavy charged particles, such as the Bragg peak, however there are some differences. As an example, the Bragg peak for heavy charged particles is narrower than that of protons (figure 1.3), because heavy particles suffer less Coulomb scattering from target atomic nuclei due to its larger mass and therefore momentum, thus enabling sharper lateral gradients to be delineated. However, heavy particles undergo a process called nuclear frag- 
mentation, which does not happen with protons, that enhances the dose beyond the Bragg peak, and thus delivering unwanted dose distal to the tumor [Crespo, 2005].

The RBE of heavy particles increases with charge up to a certain degree. For protons, a constant RBE of 1.1 is used to convert the physical dose to the biological effective dose, whereas for carbon ions the RBE increases with increasing depth in the tissue. This allows for an increased dose essentially in the tumor, while sparing the surrounding healthy tissues [Kraft, 2000].

High-LET particles or densely ionizing radiation (heavy charged particles) have additional advantages over protons. High-LET particles produce more damages in the tumor located in the Bragg peak and allow to better treat tumors that are radioresistant to low-LET particles, such as protons IAEA and ICRU, 2008]. In addition, high-LET particles damage cells mainly through direct action, which eliminates the dependence on the presence of oxygen within the cell, and cause unrepairable damage in the DNA whether there is oxygen or not. Moreover, heavy charged particles may interact with the target through a nuclear reaction, causing auto and/or target activation, decaying by $\beta^{+}$decay. This is an enormous advantage because the positrons emitted allow monitoring to some degree the dose delivered to the patient with positron emission tomography (PET) 1 Crespo, 2005; Cunha, 2010.

\subsection{Beam transport systems}

Two types of hadron accelerators (cyclotrons and synchrotrons) can be used in order to produce charged particle beams with sufficient energy to pass through a beam transport system and reach a target volume with enough intensity. For instance, figure 1.9 shows a cyclotron and a nozzle of an IBA (Ion Beam Application, Belgium) proton delivery system. In the cyclotron, the proton beam is produced with a fixed energy of $230 \mathrm{MeV}$. The proton beam is transported into treatment rooms via beam transport systems using several magnets. At the cyclotron exit, a carbon wedge is used to degrade the beam energy below $230 \mathrm{MeV}$ down to $70 \mathrm{MeV}$, while associated slits maintain the beam energy spread and divergence, i.e., spot size, within clinical requirements.

\footnotetext{
${ }^{1}$ Positron emission tomography, also called PET imaging or a PET scan, is a type of nuclear medicine imaging technique that provides medical doctors with functional information regarding body organs and tissues.
} 
Performance standards 11 of an ion therapy facility (an accelerator, delivery systems, and beam transport systems) must satisfy a number of clinical requirements Goitein, 2007]. Beam transport systems are used in order to transport the extracted beam from an accelerator to treatment radiation therapy rooms. These systems consist of bending (dipole) magnet:2 2 and focusing and defocusing (quadrapole) magnet:3, vacuum chambers, and diagnostic instrumentation. An efficient and stable transport of the beam from the accelerator to experimental setups or treatment rooms is needed to transport the given beam to the required location with the desired physical parameters. The stability of the beam position is adjusted by using focusing and defocusing magnets to control the beam position and profile [Chu et al., 1993].

\section{Beam transport system}

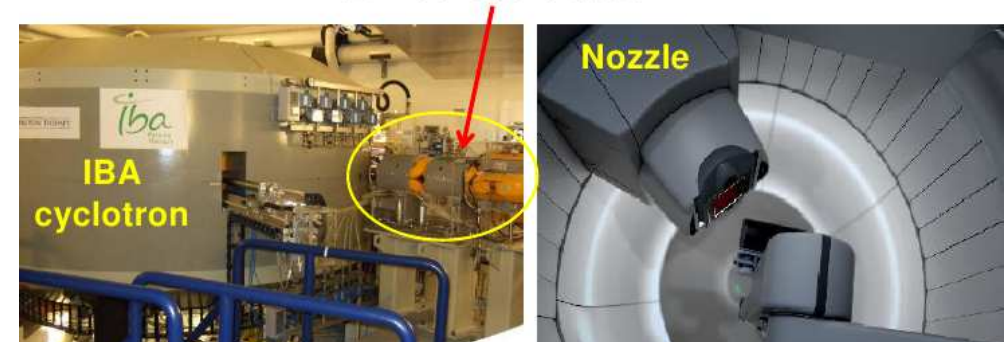

Figure 1.9: An IBA proton beam delivery system. Left image shows an IBA proton cyclotron, whereas the right image shows a gantry equipped with IBA universal nozzle inside a treatment room [Freeman, 2008].

\subsection{Beam shaping and dose delivery systems}

Beam delivery systems are located in treatment rooms; lying between beam end lines at vacuum windows and patients. Beam delivery systems have technical components and a number of devices to adapt the beam, such as modulating devices, range changing, and shaping devices, etc. Beam delivery systems are needed to conform the delivered dose to the tumor volume and to spare surrounding healthy tissues and critical structures. Two types of dose delivery systems are used (according to the method of beam spreading): (1) passive dose delivery systems (or broad-beam technique); and (2) active dose delivery systems (or pencil-beam technique).

\footnotetext{
${ }^{1}$ The performance standards of an ion therapy facility are expressed in terms of beam intensity, energy, range, distal dose fall-off, and time structure of the extracted beam, etc.

${ }^{2}$ Bending magnets deviate the charged particle beam, keeping it inside the vacuum chamber.

${ }^{3}$ Focusing and defocusing magnets control the characteristics of the transported ion beam.
} 


\subsubsection{Passive beam shaping}

Passive scattering is the most commonly used in proton and heavy ion therapy. It consists in producing a broad beam, which is further collimated transversally to conform itself to the tumor shape. The depth dose of a monoenergetic beam from an accelerator is modulated by a variable degrader, which can be a rotating wheel with varying thickness or a ride filter 1 . The modulator is designed in order to achieve the predefined depth dose profile. In order to move the modulated Bragg peak (SOBP) to the desired radiological depth, an additional range shifter is needed. The distal part of a tumor is conformed using a range compensator, at the expense of the proximal part.

To deliver a homogeneous in-depth dose distribution in the tumor, a SOBP is produced by a range modulator, which is basically a wheel with steps of variable thicknesses. Finally, the small sized beam has to be spread out laterally to cover the whole target homogeneously, which can be achieved either by a double scattering system, or by a magnetic wobbling system that moves the beam over a defined area [Schardt et al., 2010]. The principle of passive shaping is illustrated in figure 1.10.

\subsubsection{Active beam shaping}

Active scanning systems typically do not include any scattering device in the nozzle. A scheme of the active scanning technique is shown in figure 1.11. It is currently the most advanced delivery system based on a pencil beam, which is moved point-by-point to cover the whole target volume. As a result, this technique allows conforming better to the tumor shape and sparing healthy tissues. This system takes advantage of the electric charge of ions, in order to produce a tightly focused pencil beam that is then deflected laterally by two magnetic dipoles to allow a scanning of the beam over the treatment field. To conform the dose to the tumor in depth, the beam energy is modified. This technique has been used in part for the first time at the Paul Scherrer Institute (PSI) in Switzerland for proton therapy since 1996 [Soukup et al., 2005]. After, it was tested at the Gesellschaft für Schwerionenforschung (GSI) in Germany with carbon ions since 1997 Amaldi and Kraft, 2005] up to 2007. It is now under medical use at the Heidelberg Ion Beam Therapy Center (HIT, Heidelberg, Germany).

\footnotetext{
${ }^{1}$ The ride filter is a wobbling plate with shaped engravings.
} 


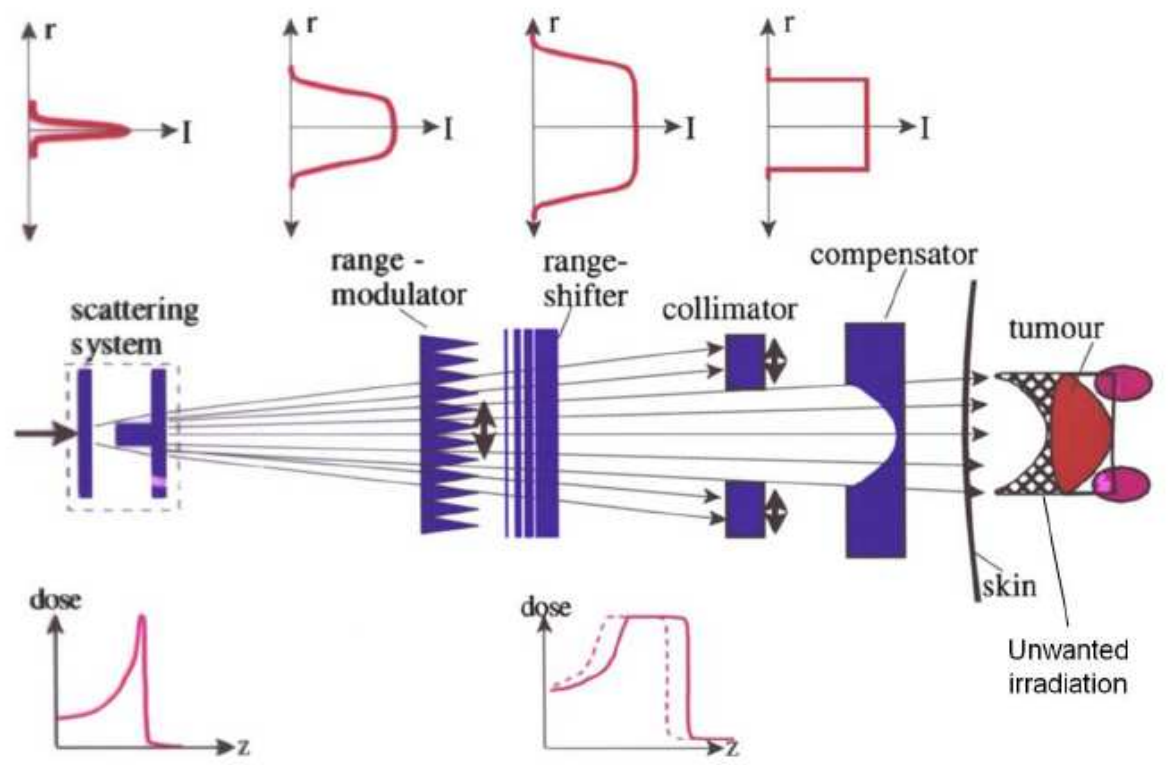

Figure 1.10: Sketch of a fully passive beam shaping system. For lateral scattering, sophisticated sets of combined absorber foils are used to reach a homogeneous dose across the target volume. The outer contours are then defined by apertures. Depth modulation is more difficult, because the depth distribution has to contain also the information of the depth-RBE dependence. Consequently, the shape of the teeth of the ridge filters determines the depth-dose distribution. Finally, compensators in front of the patient can be used to shape the distal fall off [Schardt et al., 2010].

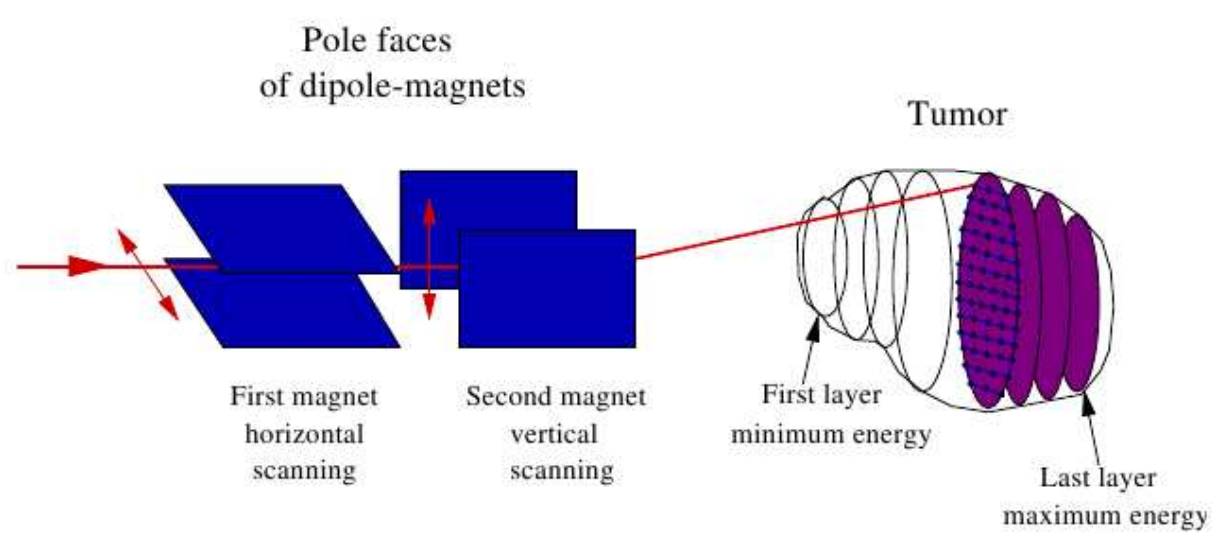

Figure 1.11: Schematic view of the active beam scanning treatment modality. The tumor is dissected into slices. Each iso-energy slice is covered by a grid of pixels for which the number of particles has been calculated beforehand. During irradiation, the beam is guided by the magnetic system in a row-by-row pattern from pixel to pixel [Haberer et al., 1993; Kraft, 2007]. 


\section{Chapter 2}

\section{State-of-the-art instrumentation for beam diagnostics}

\section{$2.1 \quad$ Introduction}

The diagnostics is a vital part of any accelerator and it is the organ of sense for the beam. Beam diagnostics allows us to understand the properties of the beam and its behavior inside accelerators. It is an important part of any accelerator for monitoring and assessing the beam. Therefore, an accelerator is just as good as its diagnostics. Additionally, beam diagnostics is not only needed for the operation of the accelerator itself, but also for its development. The beam diagnostic demands at any accelerator facility can be divided into three groups, as follows Forck, 2011; Koziol, 2010]:

1. Quick and reliable measurements leading to single numbers or simple plots in order to determine the basic parameters of the machine. The measuring instruments should be non-destructive for the beam i.e., the beam is not disturbed by the measuring apparatus. For example, beam current measurements using transformers 1 .

2. Beam instrumentation for daily check of performance and stability of the accelerator setting. These measuring instruments can also be used for malfunction diagnostics. Such instrumentation can be either destructive or non-destructive.

\footnotetext{
${ }^{1}$ See section 2.3 .1
} 
An example of a non-destructive device is a wire scanner 1 .

3. Complex instrumentation for solving hard malfunction and for the commissioning of new accelerator components. These devices can be complex and might be destructive for the beam.

After assessing beam values and determining machine parameters using beam instrumentation, a feedback i.e., active influence of the beam settings, has to be performed in order to yield an improved beam delivery.

Because the accelerating principle of linear accelerators (LINACs) differs from that of synchrotrons, their diagnostic devices and methods are different. LINACs and transfer lines are single pass i.e., the beam passes only one time through the beam chamber. On the other hand, synchrotrons are considered as multi pass, where the beam passes many times through the same beam chamber. For this reason, it is important to use non-destructive methods to monitor the beam behavior in synchrotrons. In cyclotrons, the beam is accelerated in a spiral path; therefore destructive methods for monitoring the beam can be used. For example, beam current measurements using Faraday cups 2 .

In non-destructive methods, the beam is not disturbed by the instrument and the instrument is not destroyed by the beam. The using of destructive or non-destructive methods for beam monitoring depends on the beam parameters. Another factor plays an important role in choosing the proper diagnostic devices for assessing beam parameters is the type of beam itself. If the beam is composed of electrons, we can always deal with it as relativistic beam 3 in any accelerator. However, if the beam is made up of protons, then it is considered non-relativistic if its kinetic energy less than 6.3 MeV4. Another difference is the emission of synchrotron radiation by electrons even at low energies. However, only at extremely high energies of $E>1 \mathrm{TeV}$ for protons the synchrotron radiation starts playing a certain role [Wille, 2011]. Indeed, the proton mass $\left(938 \mathrm{MeV} / \mathrm{c}^{2}\right)$ is about $2 \times 10^{3}$ times larger than that of the electron $\left(0.511 \mathrm{MeV} / \mathrm{c}^{2}\right)$. Thereby, the gamma factor 5 of a $1.5 \mathrm{GeV}$ electron beam is approximately 3000 , whereas a proton beam with $1 \mathrm{TeV}$ energy has a gamma factor of only 1,000. Therefore, the synchrotron radiation is much greater for electrons than for equal energy protons.

\footnotetext{
${ }^{1}$ See section 2.3 .4

${ }^{2}$ See section 2.2.1.

${ }^{3}$ Relativistic particle is the particle that moving close to the speed of light.

${ }^{4}$ The energies at which non-relativistic expressions for mechanical quantities of electrons and protons are in error by $1 \%$ is a reasonable threshold for relativistic treatments Nave, 2013.

${ }^{5}$ Gamma factor is the ratio of the energy of the particle to its rest mass-energy.
} 
Some beam quantities can be assessed using different techniques. On the other hand, one technique can be used for assessing different beam quantities. Beam diagnostics is a rich field covering the full spectrum of physics and technology. There exist vast choices of different types of beam diagnostic instruments, mainly based on one or more of the following physical processes [Forck, 2011]:

1. Electromagnetic influence by moving charges. This process is described by classical electromagnetics and it is based on measurements of voltage or current. For example, transformers 1 .

2. Emission of photons by accelerated charges. This process is based on optical techniques covering electromagnetic spectrum from visible light to X-rays. This technique is used only for relativistic particles, mainly electrons. For instance, synchrotron radiation monitors 2 .

3. Interaction of charged particles with matter. This diagnostics is described by atomic and solid state physics and it is based on measurements of beam current For instance, ionization chambers 3 .

4. Nuclear particle physics interactions between the incident particles and a fixed target or between colliding beams. This diagnostics is based on nuclear physics and particle detectors. For example, beam loss monitors 4 .

The beam current is one of the most important parameters for operation of particle accelerators; it is the basic quantity of the beam. The first check of accelerator functionality in almost all accelerators is done by checking the beam current. The beam current is determined in absolute manner, by displaying the average current in the form of one single number. There are different devices used to measure beam current, such as Faraday cups and ionization chambers.

\footnotetext{
${ }^{1}$ See section 2.3.1.

${ }^{2}$ Synchrotron radiation monitor is one of the non-destructive tool to easily obtain information about beam position, beam size and beam stability. This monitor diagnoses beam performance using synchrotron radiation produced when the beam traverses a bending magnet [riso and Pérez, 2006].

${ }^{3}$ See section 2.3.5.

${ }^{4}$ Particle is considered lost if it doesn't follow design trajectory and interacts with matter. Beam loss monitor is a device that detects instantaneous radiation caused by beam loss e.g., ionization chambers.
} 


\subsection{Instrumentation for destructive beam diag- nostics}

\subsubsection{Faraday cups}

In general, beam current measurements are important for many experiments that use high energetic beams. From the first days of accelerators, Faraday cups were used for current measurements. Their principle of operation is based on shooting the beam straight into a conducting plate or a cup, and conducting the current to a measuring instrument via an electrical lead Kim et al., 2010]. For high current beams ( $\gtrsim 10 \mathrm{~mA}$ ) this destructive method cannot be applied, because the total energy carried by the beam can damage the cup. Nevertheless, low current beams can be fully determined using this destructive method. A measurement of $10 \mathrm{pA}$ is possible with a low noise current-to-voltage amplifier and careful mechanical design. For higher energetic particles, the range of the beam can be more than several $\mathrm{cm}$ and Faraday cups are not useful any more.

Figure 2.1, left shows schematically the design of a Faraday cup, which consists of an isolated metal chamber that intercepts the charged particle beam, the cup being connected to an electrical lead which conducts the current to a measuring equipment. Secondary electrons are liberated from the metal cup when accelerated charged particles hit its surface. The energy of liberated electrons is on average $10 \mathrm{eV}$. The liberated electrons can escape into the surroundings and probably leave the metal cup. As a result, secondary electrons leaving the cup would result in wrong current measurements. There are three ways to prevent the liberated secondary electrons from leaving the isolated metal cup, as following [Forck, 2011; Raich, 2005]:

1. Designing the metal cup by making the length of the cup in the beam direction much longer than the cup diameter. For this long metal cup, the loss of secondary electrons is low.

2. Applying a high voltage to the suppression ring close to the entrance of the metal cup, as shown in figure 2.1. Since the liberated secondary electrons have very low energies of less than $20 \mathrm{eV}$, a polarization voltage of some $100 \mathrm{~V}$ is sufficient to prevent them from leaving the cup.

3. Applying a magnetic field to the surface of the metal cup by using permanent magnets, as shown in figure 2.1. In this case, the secondary electrons move 


\subsection{Instrumentation for destructive beam diagnostics}
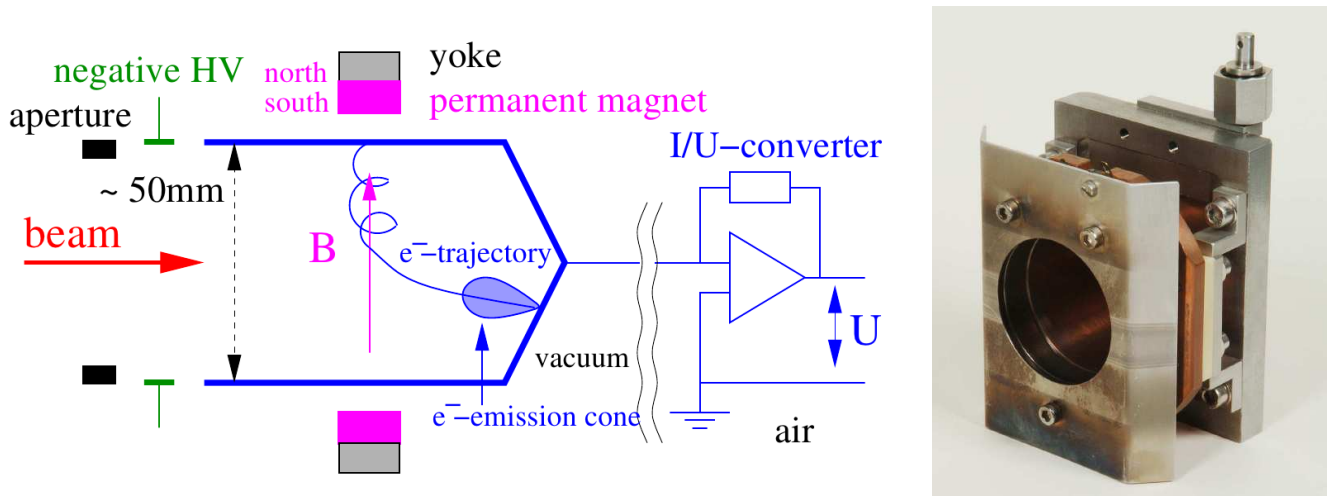

Figure 2.1: Left: Schematic diagram of a Faraday cup with magnetic and electric suppression of secondary electron emission. It shows a metal cup (blue) and a measuring electronics (current-to-voltage converter). Right: Photograph of a Faraday cup [Forck, 2011].

in spiral motion around the magnetic field lines with a cyclotron radius $r_{c}$ given in equation 2.1.

$$
r_{c}[m m]=\frac{\sqrt{2 m_{e} E_{k i n}}}{e B}=3.37 \frac{\sqrt{E_{k i n}[e V]}}{B[m T]}
$$

where $B$ is the magnetic field, $m_{e}$ is the electron mass, $e$ its charge, and $E_{k i n}$ being the perpendicular component of the electron kinetic energy.

Usually, Faraday cups operate in vacuum, but some experiments require measuring beam current outside the chamber of an accelerator. For this reason, an external Faraday cup has been developed by Kim et al. [2010] for low-flux experiments with proton beam energy range up to $45 \mathrm{MeV}$. This cup is composed of a vacuum chamber, an entrance window, a collimator, an electrostatic suppressor ring, and a metal cup. The vacuum pressure inside the chamber during beam measurements is on average $5 \mathrm{mTorr}$.

\subsubsection{Scintillation-based devices}

A scintillator is material that exhibits the property of luminescence when excited by ionizing radiation. Scintillating materials when struck by a particle, absorb its energy and scintillate i.e., re-emit the absorbed energy in the form of light, typically in the visible range. Luminescent materials can emit light via two processes. The first one is characterized by a direct jump of the excited electrons 
to the base level with a short decay time constant of a few tens of nanoseconds or more precisely within $10^{-8} \mathrm{~s} 1$, this process is called fluorescence. In the second process, the de-excitation passes via an intermediate receiver level and takes much longer, typically microseconds to seconds. This process is called phosphorescence. If both processes are present, the light emission is called luminescence. Therefore, the essential difference between fluorescence and phosphorescence is just a matter of time [Ahmed, 2007; Knoll, 2000; Leo, 1994.

The four main factors on which the generated light yield of a scintillator depends are: (1) the scintillation material; (2) the type of radiation; (3) the energy of the incident particles; and (4) temperature. Desirable properties of a good scintillator are listed in the following points [Ahmed, 2007; Knoll, 2000]:

1. High conversion efficiency 2 i.e., the produced light should be linear to the energy deposited in the scintillator material.

2. Large dynamic range i.e., a good linearity between the incident particle flux and the output light.

3. Decay time 3 of excited states of the scintillator material should be short. Thereby, fast signal pulses can be generated.

4. The scintillator material should be transparent to its own emission for good light collection.

5. The scintillator material should be easy to form and it should be possible to get in large sizes.

6. The scintillator material should be radiation hard, so it will not be destroyed by the incident particles in short time.

7. The wavelength of the scintillation light should be within the spectral range of the photocathodes of photomultiplier tubes (PMTs 4) and within the spectral

\footnotetext{
${ }^{1} 10^{-8} \mathrm{~s}$ being roughly the time taken for atomic transition.

${ }^{2}$ The effect of decrease in light output of a scintillator due to change in some parameter such as temperature, energy of the incident radiation, or impurity in the scintillator material is called quenching.

${ }^{3}$ Most scintillators produce pulses with very fast rise times (less than $1 \mathrm{~ns}$ ). However, the decay time of typical scintillation pulse can be as low as a few nanoseconds and as high as several milliseconds.

${ }^{4}$ Photomultiplier tubes perform two functions: (1) covert ultraviolet and visible light photons into an electrical signal; and (2) amplify the produced signal, on the order of millions. A photomultiplier tube consists of an evacuated glass tube containing a photocathode, followed
} 
range of charge-coupled devices (CCDs).

\subsubsection{Thin scintillator readout by $\mathrm{PMT}$}

The scintillation detector is one of the widely used detection devices in nuclear and particle physics. In scintillation detectors, when a particle penetrates scintillating material, the electronic energy loss from the collisions between beam particles and target electrons creates some fluorescent light. This light can be detected and amplified using a photomultiplier tube (PMT). In other words, it can be converted to electrical pulses which can be analyzed and counted electronically to give information about the incident radiation. A scintillation counter (figure 2.2) consists of a scintillation material, a light guide, and a device such as PMT that converts scintillation light into an electrical signal [Leo, 1994].

\subsubsection{Thin scintillator readout by CCD}

A fluorescent screen is a sheet of material coated with fluorescent substance. Therefore, it emits light when struck by ionizing radiation such as a proton beam. The beam spot can be directly observed by intercepting the beam with a scintillator screen and viewing the emitted light with a CCD camera. Scintillation screens are the most direct way of beam profile observation as used from the early days on. These devices are installed in nearly all accelerators from the source up to the target. Figure 2.3, left shows schematically a scintillation screen. It is moved into the path of the beam, and usually it is inclined at an angle of $45^{\circ}$ to the beam direction as shown in figure 2.3. At an angle of $90^{\circ}$ to the beam direction, a CCD camera is used to see the two dimensional image of the beam cross-section where the beam density information can be extracted Bal et al., 2005; Forck, 2011; Koziol, 2010.

The disadvantage of scintillation screens is related to the interception of the beam. However, the main advantages are listed in the following points [Forck, 2011]: (1) direct two dimension measurement of the beam profile; (2) high spatial resolution; (3) cheap realization; and (4) observation with a CCD camera with digital output or video.

Several materials can be used in scintillation screens. Table 2.1 summarizes some of these inorganic material and their basic properties. In order to compare

by typically 10 to 12 electrodes called dynodes, and an anode Hamamatsu Photonics K. K., 2006. 


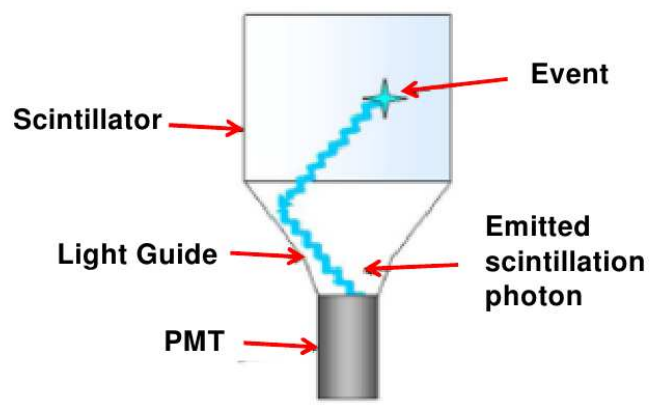

Figure 2.2: Schematic diagram of a plastic scintillation counter connected to a photomultiplier tube via a plastic light guide.
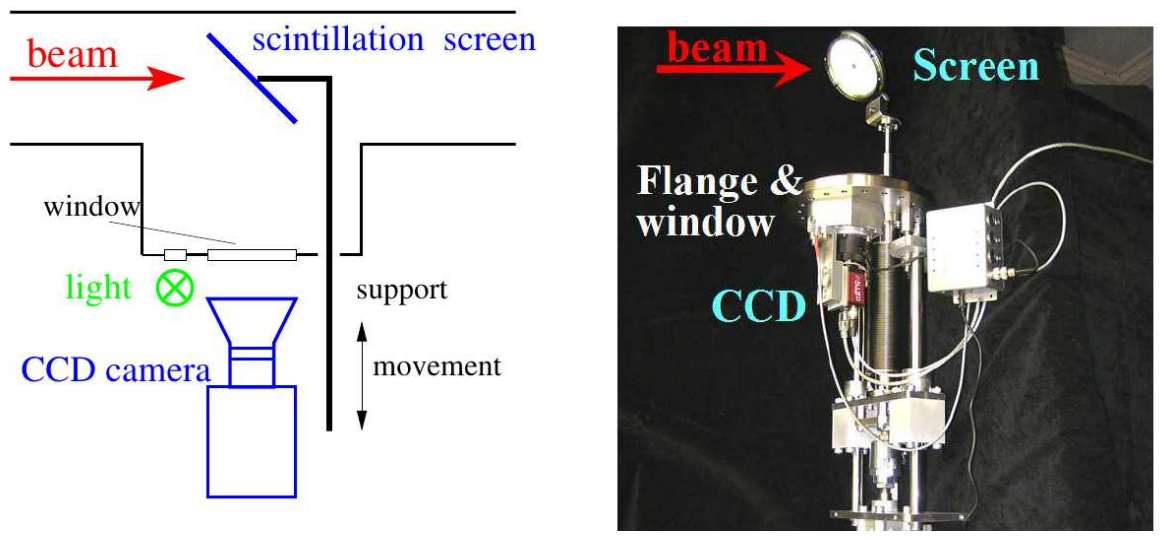

Figure 2.3: Schematic diagram (left) and photograph (right) of an intercepting scintillation screen taped by a CCD camera [Forck, 2011].

the response of various scintillating materials to an incident beam, it is possible to use a monitor having a screen holder fitted with different screen types, as shown in figure 2.4.

The matrix $\mathrm{Al}_{2} \mathrm{O}_{3}$ (Chromolux) is a ceramic in form of glass. This material has a high resistance against radiation damage and temperature caused by the beam, therefore it is commonly used [Jung et al., 2003]. The disadvantages of the Chromolux are: Its long decay time of about $100 \mathrm{~ms}$ and large absorption of its own emitted scintillation light.

\subsubsection{Plastic scintillators}

Plastic scintillators offer high performance, ease of handling, and mechanical stability at relatively low cost, consequently the use of plastic scintillators is very 


\subsection{Instrumentation for destructive beam diagnostics}

Table 2.1: Properties of common inorganic scintillators. The last four materials are so-called phosphor screens, where a powder is deposited on glass or metal plates [Forck, 2011; Knoll, 2000].

\begin{tabular}{|l|l|l|l|l|}
\hline Abbreviation & Material & Activator & Max. emission & Decay time \\
\hline Quartz & $\mathrm{SiO}_{2} / \mathrm{CsI}$ & None / Tl & Optical / 550 nm & $<10 \mathrm{~ns} / 1 \mu \mathrm{s}$ \\
\hline Chromolux & $\mathrm{Al}_{2} \mathrm{O}_{3}$ & $\mathrm{Cr}$ & $700 \mathrm{~nm}$ & $100 \mathrm{~ms}$ \\
\hline & $\mathrm{Li}$ glass & $\mathrm{Ce}$ & $400 \mathrm{~nm}$ & $0.1 \mu \mathrm{s}$ \\
\hline $\mathrm{P} 11$ & $\mathrm{ZnS}$ & $\mathrm{Ag}$ & $450 \mathrm{~nm}$ & $0.2 \mu \mathrm{s}$ \\
\hline $\mathrm{P} 43$ & $\mathrm{Gd}_{2} \mathrm{O}_{2} \mathrm{~S}$ & $\mathrm{~Tb}$ & $545 \mathrm{~nm}$ & $1 \mathrm{~ms}$ \\
\hline $\mathrm{P} 46$ & $Y_{3} \mathrm{Al}_{5} \mathrm{O}_{12}$ & $\mathrm{Ce}$ & $530 \mathrm{~nm}$ & $0.3 \mu \mathrm{s}$ \\
\hline $\mathrm{P} 47$ & $Y_{2} \mathrm{Si}_{5} \mathrm{O}_{5}$ & $\mathrm{Ce}, \mathrm{Tb}$ & $400 \mathrm{~nm}$ & $100 \mathrm{~ns}$ \\
\hline
\end{tabular}

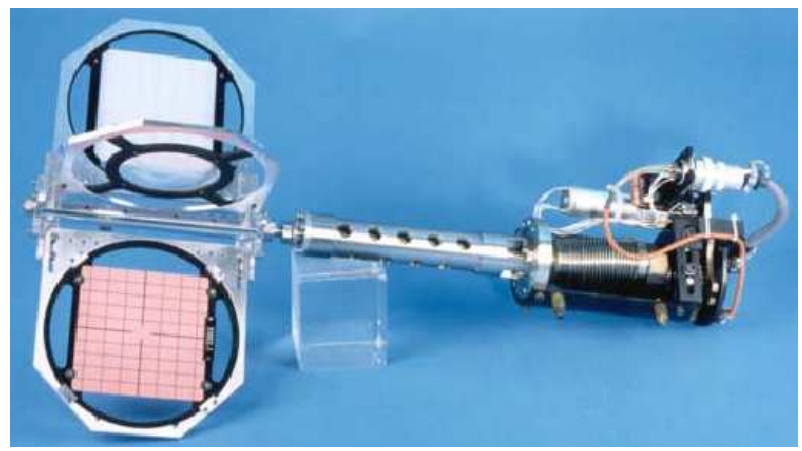

Figure 2.4: Screen holder with three screens (from bottom to top: Cr-doped $\mathrm{Al}_{2} \mathrm{O}_{3}$, Tl-doped CsI, and Quartz) and one empty position for the free passage of the beam [Jung et al., 2003].

attractive. Plastic scintillators can be made up of a solid solution of organic scintillating molecules in a polymerized solvent. Because of the ease with which they can be shaped and fabricated, they have become an extremely useful form of organic scintillator. Some plastic scintillators are characterized by short decay time, on the order of nanosecond. This makes the material well suited for fast timing measurements. The disadvantage of the plastic scintillators is the low radiation hardness, due to their complex organic molecular structure. Inorganic crystals have much higher radiation hardness. However, these scintillators are difficult to produce in larger sizes and in different mechanical shapes, because they consist of single crystals [Ahmed, 2007; Knoll, 2000; Leo, 1994.

The major problem associated with plastic scintillators is their nonlinear behavior i.e., they do not respond linearly to the ionization density. The light yield 
per path length $d L / d x$ as a function of the energy loss per path length $d E / d x$ for a particle traversing a scintillator is not linear at high loss rates. This behavior is characterized by the so called Birks' formula (equation 2.2), which posits that recombination and quenching effects between the excited molecules and the surrounding substrate reduce the light yield [Ahmed, 2007; Birks, 1951]:

$$
\frac{d L}{d x}=\frac{A d E / d x}{1+k d E / d x}
$$

where $A$ is the absolute scintillation efficiency and $k$ is Birks' constant, which must be determined for each scintillator by measurement.

The light emission wavelength and the decay time depend on the type of organic activator and on the host material. Plastic scintillators are commercially available in a wide selection of standard sizes such as Pilot U [Eljen Technology team, 2013].

\subsubsection{Thermoluminescent dosimetry}

The term thermoluminescence stands for thermally stimulated luminescence i.e., emission of light by thermal stimulation. Thermoluminescent (TL) materials have the ability to store energy delivered by ionizing radiation and then release it after being exposed to heat i.e., after their temperatures are raised above a certain level. Thermoluminescent materials are used as radiation detectors since 1950s. Since that time such detectors are being extensively used in personal dosimetry applications. Thermoluminescent dosimetry is one of the commonly used methods for measured integrated dose. The thermoluminescent material is used to record dose and then it is taken out of the radiation environment to read the recorded value of dose.

The band theory of solids can be used to explain thermoluminescence phenomenon. Thermoluminescent materials consist of crystals to which a small concentration of impurity is added as an activator e.g., $\mathrm{CaSO}_{4}: \mathrm{Mn}$, where the manganese is the activator. Some others do not require the addition of an activator. TL materials are exhibited by host of materials such as glasses, plastics, and some organic solids. The crystal defects and impurities in TL materials can produce energy levels inside the forbidden gap. Such levels, which act as electron traps, can be used to store information in TL materials. When a TL material is irradiated by ionizing radiation, electrons and holes are produced, as shown in figure 2.5. The incident radiation elevates electrons from the valence band to 


\subsection{Instrumentation for destructive beam diagnostics}

the conduction band and leaves behind vacancy holes in the valence band. During irradiation, electrons can be trapped at the defect sites near the conduction band i.e., a metastable site. When the TL material is heated, these trapped electrons get enough thermal energy to escape from the trap to the conduction band. From here they may get re-trapped again at impurity levels near the valence band and emit photons during this process. The electrons in impurity levels near the valence band finally falls into the valence band and recombine with the holes [Ahmed, 2007; Gei, 2002].

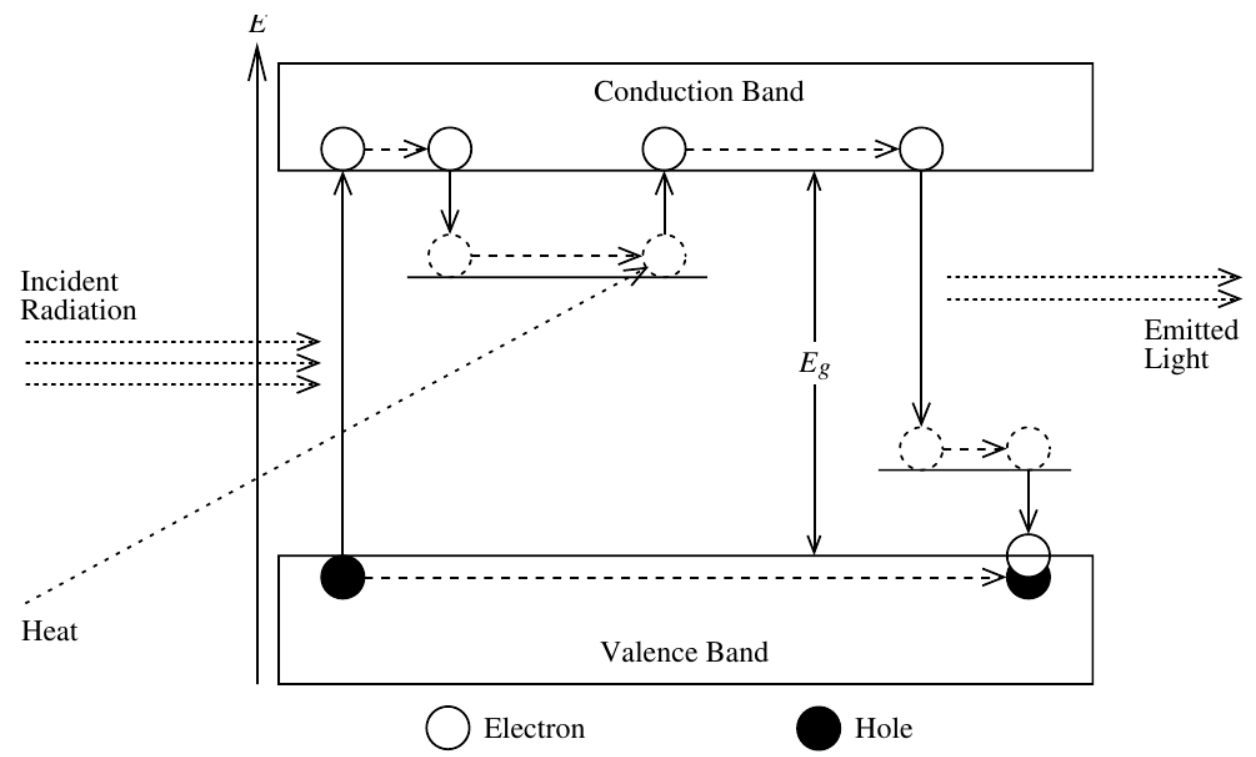

Figure 2.5: A simple model of energy absorption in a TL material. This diagram shows processes involved in radiation induced electron/hole trapping and subsequent recombination on thermal stimulation with associated light photon emission [Ahmed, 2007].

The phenomenon of thermoluminescence is exploited in detectors called thermoluminescent detectors (TLDs), which are memory devices used to monitor absorbed dose over a period of time. These devices have been highly successful because of their following advantages: (1) wide useful dose range; (2) small physical size; (3) there is no need for high voltage or cables 1; and (4) their tissue equivalence for most radiation types. The disadvantages of these materials are: (1) they can not be used to measure instantaneous doses; and (2) they are not as accurate as electronic detectors [Ahmed, 2007; Janovsky and Ross, 1993; Knoll, 2000].

\footnotetext{
${ }^{1}$ Thermo-Luminescent Detectors (TLDs) do not need any electronic circuitry for operation and can be easily installed and retrieved.
} 
An example of a thermoluminescent dosimeter is lithium fluoride. A small crystal of lithium fluoride $(L i F)$ containing a small quantity of manganese as an impurity is placed into a holder. The TLD crystal absorbs and stores the energy from ionizing radiation that strikes it. When heated, this stored energy is released from the crystal in the form of light. When reading the TLD crystal, it is placed in a dark chamber equipped with a photomultiplier tube (PMT) (figure 2.6). When the crystal is heated, the photomultiplier converts the light into an electronic signal which then is amplified and on through the system for analysis. The resulting output of the TLD recorder is called a glow curve and the area under this curve is directly proportional to the absorbed dose in the TLD crystal [Ahmed, 2007; Gajewski, 2013].

Hsu et al. 1998 described the response of the thermoluminescent dosimeter CaF2 : Tm (TDL-300) to protons with energies between 15 and $30 \mathrm{MeV}$. Glow curve analysis indicates that the response of $C a F 2: T m$ to protons depends on beam energy and beam current.

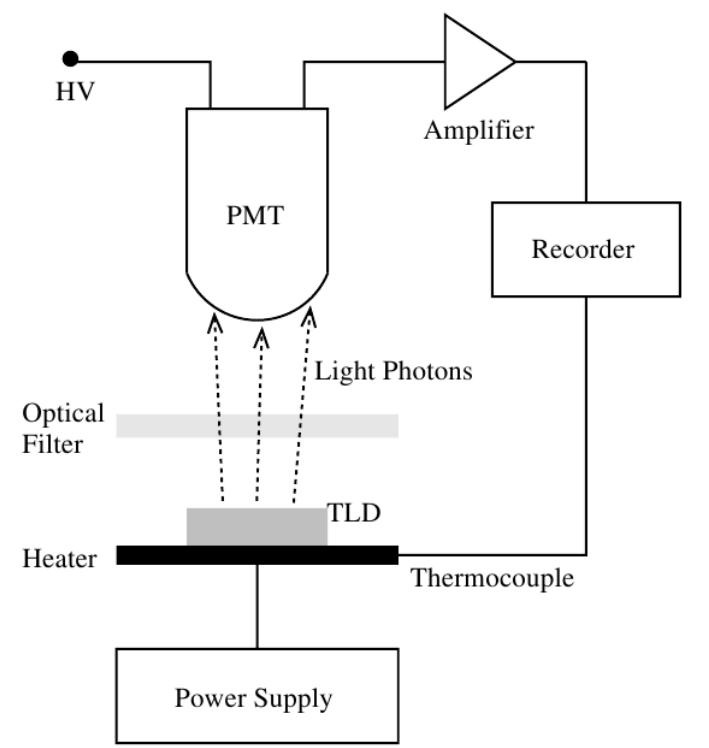

Figure 2.6: Schematic diagram of a simple TLD readout system. It shows a power supply, a heater, a thermocouple, a TLD, an optical filter, a PMT, and a recorder [Ahmed, 2007].

\subsubsection{Film dosimetry}

Radiographic films used in diagnostic radiology were used widely before affordable radiochromic films were introduced. The former were based on silver halide 
film technology from the photographic industry. Radiographic films were designed primarily for detecting low energy keV radiation. These films have two disadvantages: (1) the need for dedicated developers and darkrooms; and (2) the presence of silver causes strong energy dependence of the film response to radiation. For quantitative dose measurements in $\mathrm{MeV}$ beams, radiochromic films were developed. The radiochromic film consists of a single or double layer of radiation-sensitive organic microcrystal monomers, on a thin polyester base with a transparent coating [Kirby, 2011].

Film dosimetry is based on the so called radiochromatic materials, which change color when exposed to ionizing radiation. The amount of this coloration is proportional to the delivered dose i.e., the darkness of the irradiated films increase with increasing absorbed dose. Therefore, radiochromatic films provide a direct way of dose measurements. The degree of film coloring is measured by the optical density of the film (OD) (equation [2.3): with OD representing the reduction in the intensity of light that is transmitted through the film when a white light source is shone on it after irradiation. Since white light is composed of photons of different wavelengths, its transmission through the film depends on the absorption coefficient at each wavelength. Thus the film appears colored.

$$
O D=\log \left(I_{0} / I\right)
$$

where $I_{0}$ is the light intensity with no film present, and $I$ is the light intensity that passes through the film [Ahmed, 2007; Butson et al., 2003]. The reason for defining optical density in this way is that the ratio $I_{0} / I$ has an exponential dependence on dose $D$. Therefore, the relationship between $O D$ and dose is usually close to linear in the main range of operation.

Radiochromic films do not require a developer or a darkroom facility like radiographic films EBT2 team, 2009]. Earlier versions of radiochromic films such as HD-810 (formerly: DM-1260) has nominal dose range from 10 Gy up to 1000 Gy [Soares, 2013]. Radiochromic films were limited to industrial irradiation facility use. As film production technology improved, radiochromic films like the MD-55 were introduced to clinical and research environments for $\mathrm{MeV}$ beams. The nominal dose range of MD-55 films is $1-100$ Gy Soares, 2013. However, across a sheet of MD-55 film, the sensitivity varies by up to $15 \%$. Such sensitivity variation makes these films unattractive for accurate dose distribution measurement. Gafchromic ${ }^{\circledR}$ external beam therapy (EBT) films were released for the first time in 2004 by the International Specialty Products (ISP, Wayne, NJ). These films are the first type of radiochromic films suitable for use with doses as low as the typical doses occurring in radiation therapy. The nominal dose 
range of Gafchromic ${ }^{\circledR}$ EBT films ranges from 0.05 Gy up to 100 Gy. In 2009, the Gafchromic ${ }^{\circledR}$ EBT film was replaced by Gafchromic ${ }^{\circledR}$ EBT2 that incorporates a yellow marker dye in the active layer and a synthetic polymer as the binder component. The nominal dose of Gafchromic ${ }^{\circledR}$ EBT2 films ranges from $0.01 \mathrm{~Gy}$ up to 40 Gy [ISP team, 2010].

In 2011 a new generation of radiochromic films, Gafchromic ${ }^{\circledR}$ EBT3, has become available. Gafchromic ${ }^{\circledR}$ EBT3 has the same composition and thickness of the sensitive layer of the previous Gafchromic ${ }^{\circledR}$ EBT2 film, but its symmetric layer configuration allows the user to eliminate side orientation dependence, which is reported for EBT2 films. Additionally, it makes the product more robust and allows water immersion Borca et al., 2013].

The different models of Gafchromic ${ }^{\circledR}$ films are relatively new films developed specifically for absorbed dose measurement of high energy photons, to speed up the workflow and to improve the spatial accuracy of dosimetric measurements. These films are used for daily quality assurance measurement of beam parameters such as beam position and beam size. Because of their high spatial resolution in two dimensions of less than $0.1 \mathrm{~mm}$, tissue equivalence, energy independence for photons, electrons and protons, and high sensitivity, Gafchromic ${ }^{\circledR}$ films are promising candidates for many applications. The alignment of proton beam verification in many facilities can be performed using these films. The longitudinal and the lateral proton beam profiles can be assessed using Gafchromic ${ }^{\circledR}$ films [Kirby et al., 2010; Martisíková and Jäkel, 2010].

Gafchromic ${ }^{\circledR}$ films are attractive in radiotherapy because of their self developing behavior. In addition, they can be handled and prepared in visible light and they do not need any chemical or physical processing. This property eliminates the needs for darkrooms. This feature is not available for the other kinds of films but it is still recommended to keep the films in dark when they are not in use [Zhao and Das, 2010]. Furthermore, Gafchromic ${ }^{\circledR}$ films can be immersed in water phantoms since the active layer is protected by two polyester substrates. In addition, they withstand temperatures up to $70^{\circ} \mathrm{C}$. The films may be stored at room temperature but it is better to store them at refrigerator temperature. It is recommended that the film be handled and prepared at room temperature. Gafchromic ${ }^{\circledR}$ films are available in large size and they are convenient to handle and can be easily cut to required shapes and sizes. Moreover, they can be marked with marker pen as needed since the outer layers are polyester EBT2 team, 2009; Kirby, 2011; Martisíková and Jäkel, 2010].

Gafchromic ${ }^{\circledR}$ films are constructed of clear, polyester outer layers which sand- 
Table 2.2: Nominal atomic abundances of Gafchromic ${ }^{\circledR}$ EBT2 film, based on data received from the International Specialty Products (ISP. Wavne. NJ) [EBT2 team, 2009].

\begin{tabular}{|c|c|c|}
\hline Element & Symbol & Atomic abundances (\%) \\
\hline Carbon & $\mathrm{C}$ & 42.37 \\
\hline Hydrogen & $\mathrm{H}$ & 40.85 \\
\hline Oxygen & $\mathrm{O}$ & 16.59 \\
\hline Lithium & $\mathrm{Li}$ & 0.10 \\
\hline Chlorine & $\mathrm{Cl}$ & 0.04 \\
\hline Nitrogen & $\mathrm{N}$ & 0.01 \\
\hline Potassium & $\mathrm{K}$ & 0.01 \\
\hline Bromine & $\mathrm{Br}$ & 0.01 \\
\hline
\end{tabular}

wich two sensitive layers of microcrystalline diacetylene suspended in gelatin Rink, 2008]. Irradiation of Gafchromic ${ }^{\circledR}$ EBT2 films causes a solid-state polymerization via a free-radical mechanism, and the newly formed polydiacetylene chains exhibit a black color, the blackness of which correlates to the dose deposited in the sensitive layers Kirby et al., 2010]. Figure 2.7, right, shows the configuration of a Gafchromic ${ }^{\circledR}$ EBT2 film, which is made by laminating an active film coating by adhesive layers, where a $30 \mu \mathrm{m}$ active layer is coated on $175 \mu \mathrm{m}$ polyester layer. The over laminate consists of $50 \mu \mathrm{m}$ polyester layer and $25 \mu \mathrm{m}$ adhesive layer [EBT2 team, 2009; ISP team, 2010]. The atomic abundances of a Gafchromic ${ }^{\circledR}$ EBT2 film are listed in table 2.2. The density of Gafchromic ${ }^{\circledR}$ EBT2 was found to be $1.2224 \mathrm{~g} / \mathrm{cm}^{3}$ using SRIM/TRIM simulation toolkit [Ziegler, 2013].

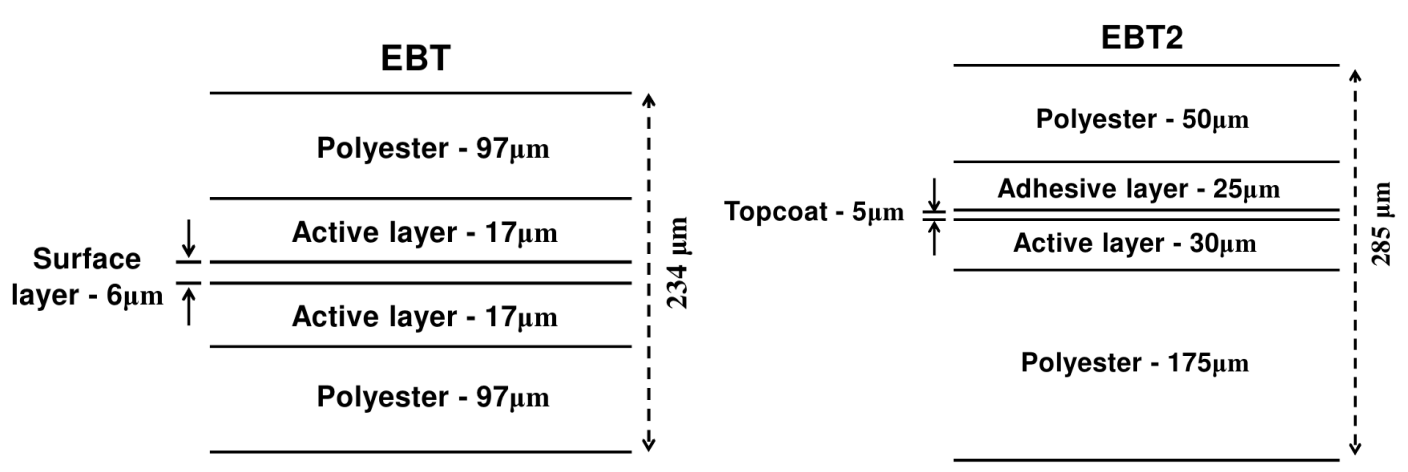

Figure 2.7: Configuration of Gafchromic ${ }^{\circledR}$ EBT (left) and EBT2 (right) dosimetry films. 


\subsubsection{Semiconductor-based devices}

A semiconductor detector (solid state detector) is a device that uses a semiconductor to detect both traversing charged particles and photons. These detectors are fundamentally different from scintillation detectors 1; they are based on detection of charge carriers (electrons and holes) generated in semiconductors by the energy deposited from ionized particles [Leo, 1994].

In a semiconductor detector, the radiation is measured by means of number of charge carriers set free in the detector. An electric field is applied to the detector volume using two electrodes. Figure 2.8 shows the energy band structure of insulators, semiconductors, and conductors. The band gap in insulators and conductors are opposite to each other, being very large for insulators $(\sim 6 \mathrm{eV})$ and non-existent for conductors. Semiconductors, on the other hand, have a small band gap, the smaller the band gap, the easier it is for electrons to move to the conduction band. An electron in the semiconductor is fixed in its valence band till a radiation interaction provides the electron enough energy to move to the conduction band, thereby ionizing radiation produces free electrons and holes [Ahmed, 2007]. The number of electron-hole pairs is proportional to the energy transmitted by the radiation to the semiconductor [Knoll, 2000]. As a result, a number of electrons are transferred from the valence band to the conduction band, and an equal number of holes are created in the valence band. Electrons in the conduction band can respond to the electric field between the two electrodes of the detector, and therefore move to the positive contact that is creating the electrical field. When the electrons are drifted to the positive voltage, the holes are drifted to the other direction, producing a current in the detector. The electrical signal is sent to a preamplifier and on through the system for analysis. As the amount of energy required to create an electron-hole pair is known, and it is independent of the energy of the incident radiation, measuring the number of electron-hole pairs allows the energy of the incident radiation to be assessed. Common semiconductor-based detectors include silicon, germanium, and diamond [Ahmed, 2007; Leo, 1994; Lutz, 1999].

Semiconductor detectors have several advantages listed in the following points Knoll, 2000; Lutz, 1999]:

1. High energy resolution. The energy required for production of electron-hole pairs is very low compared to the energy required for production of electron-ion

\footnotetext{
${ }^{1}$ See section 2.2 .2 .1
} 


\subsection{Instrumentation for destructive beam diagnostics}

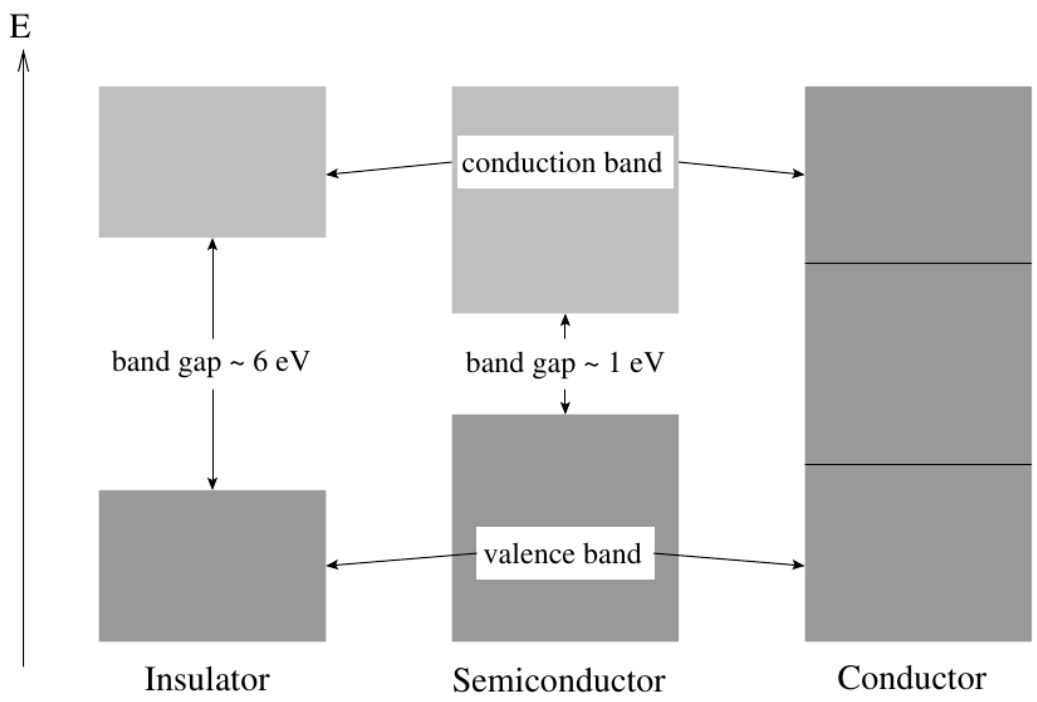

Figure 2.8: Simplified energy band structure diagrams for insulators, semiconductors, and conductors. The electronic configuration of atoms consists of a valence band where atomic electrons reside, a forbidden band, and a conduction band. In semiconductors, the forbidden band is on the order of $1 \mathrm{eV}$, where electrons with enough energy can jump to the conduction band. As temperature of the material is increased, electrons gain more thermal energy and it is easier for them to jump to the conduction band [Ahmed, 2007].

pairs in a gas detector 1 , for example.

2. Relatively fast timing characteristics. The time resolution is very good since electrons travel very fast in the conduction band.

3. Compact size. Compared with gaseous ionization detectors, the density of a semiconductor detector is very high, and charged particles of high energy can give off their energy in a semiconductor of relatively small dimensions.

4. The effective thickness of the detector can be varied to match requirements of the applications.

\subsubsection{Silicon-based semiconductor}

Semiconductor detectors can be used as particle counters. For charged particles detection, silicon is the most widely used semiconductor material. In typically

\footnotetext{
${ }^{1}$ See section 2.3 .5
} 
about $100 \mu \mathrm{m}$ thick depletion layer of doped silicon crystal, electron-hole pairs are generated by the energy deposited from the ionized particles (figure 2.9). Electrons and holes are swept away under the influence of the electric field and a proper electronics can collect the charges [Forck, 2011].

Most silicon particle detectors work in principle by doping narrow (nearly $100 \mu \mathrm{m}$ wide) strips of silicon to make them into diodes, which are then reverse biased. As charged particles pass through these strips, they cause small ionization currents which can be detected and measured. Silicon detectors have a much higher resolution in tracking charged particles than older technologies e.g., ionization chambers. The disadvantage of these detectors is that they are much more expensive than those older technologies [Knoll, 2000].

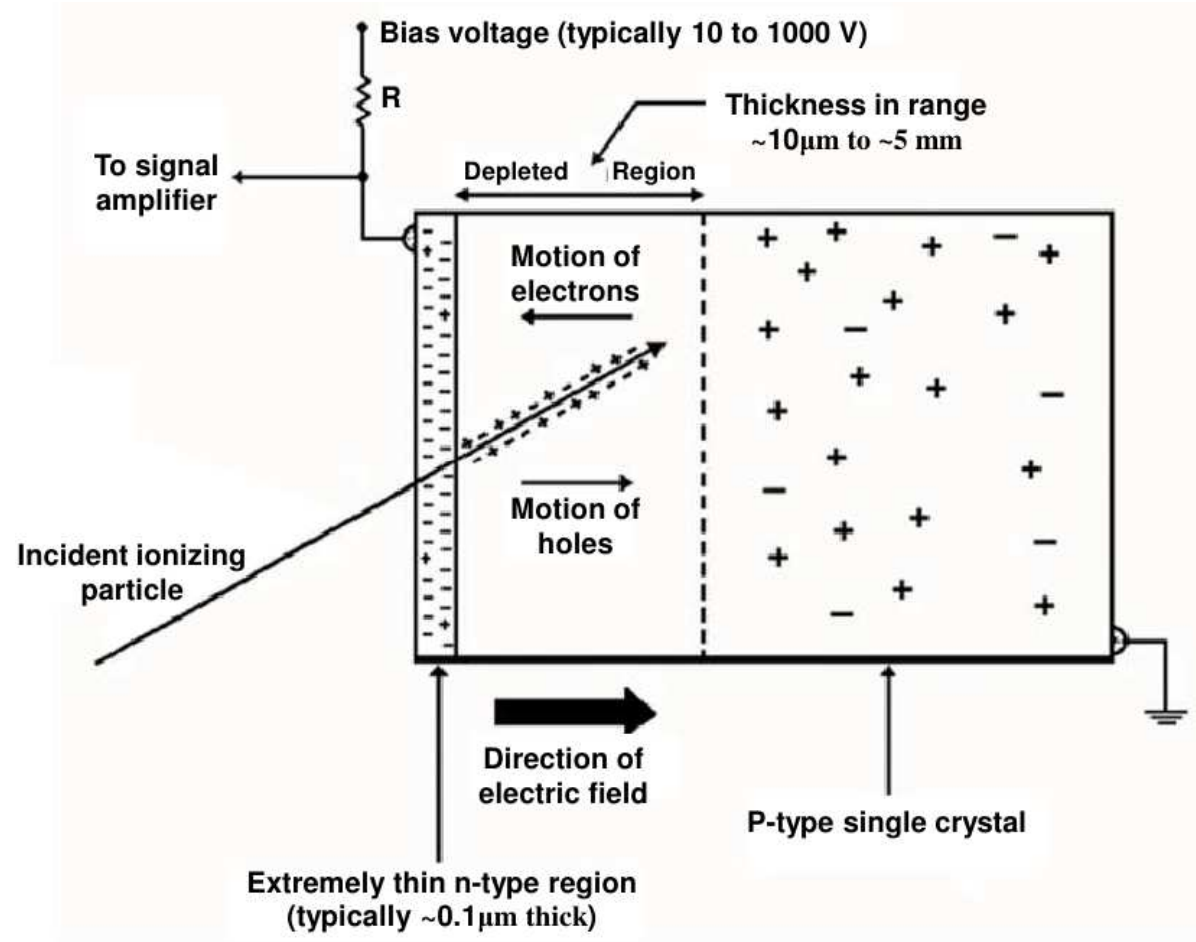

Figure 2.9: Schematic diagram of semiconductor detector (silicon-based detector). Radiation incident upon the semiconducting junction produces electron-hole pairs as it passes through it. Electrons and holes are moved away under the influence of the electric field. The sensitive volume of the semiconductor detector (depleted region) is an electronically conditioned region in the semiconductor material in which liberated electrons and holes move freely [NSEP team, 2011].

The silicon-based semiconductor has several desirable features listed in the following points [Ahmed, 2007; Knoll, 2000; Lutz, 1999]: 


\subsection{Instrumentation for destructive beam diagnostics}

1. Semiconductor with moderate band gap of $1.12 \mathrm{eV}$.

2. Room temperature operation. The thermal energy of silicon semiconductor is $25 \mathrm{meV}$ i.e., little cooling for silicon-based detectors is typically required.

3. The energy required for creating an electron-hole pair is $3.6 \mathrm{eV}$. This value of energy for electron-hole pair creation is low when compared to the energy required to create an electron-ion pair in an ionization chamber 1. Consequently, the carriers yield in silicon-based detectors is higher than that in ionization chambers, and thereby the energy resolution is better.

4. The carrier mobility is high. Therefore, the time needed to collect the entire signal is typically less than $30 \mathrm{~ns}$.

\subsubsection{Diamond-based semiconductor}

Diamond-based detectors have many similarities with silicon-based detectors, however diamond-based detectors offer significant advantages, in particular high radiation hardness 2 , i.e., less vulnerability to radiation damage. Using diamond as a detector is not a new technology and as early as the 1920s highly selected natural diamonds were being used for ultraviolet (UV) light detection. The recent commercial availability of high purity single crystal diamond with excellent bulk uniformity is however a new development. Diamond-based detectors (figure 2.10) are much more expensive and more difficult to manufacture when compared to silicon-based detectors.

There are several reasons for choosing diamond to be used as a detector, which are listed in the following points Breidenbach et al., 2004; Stolz, 2013; Tapper, 2000]:

1. High sensitivity i.e., low absorbance gives great throughput, improving the detector sensitivity.

2. High temperature operation without the needs for cooling because diamond has high thermal conductivity.

\footnotetext{
${ }^{1}$ For example, the energy required to create an electron-ion pair (W-value) in an ionization chamber that contains argon in its active region is $26 \mathrm{eV}$. See section 2.3.5.

${ }^{2}$ For diamond the main damaging effect of radiation is the production of energy levels inside the band gap. These gaps can act as electron traps and thus introduce non-linearity and degradation in the detector response.
} 


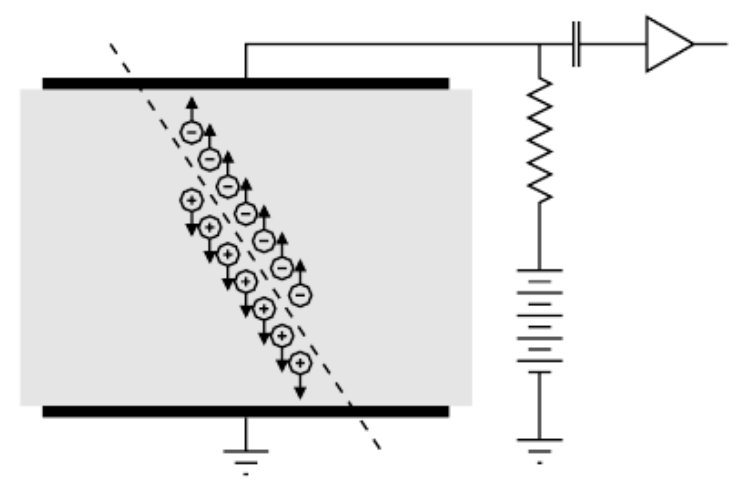

Figure 2.10: Sketch of a simple diamond-based detector [Tapper, 2000].

3. Radiation hard up to $10^{15}$ protons $/ \mathrm{cm}^{2}$.

4. Wide band gap of $5.5 \mathrm{eV}$. Consequently, there is no noise generated thermally i.e., diamond can be used at room temperature.

5. High hole and electron mobilities. The high mobility of free charges in diamond is a positive factor for radiation detection since it implies fast charge collection and consequently fast overall response of the detector.

6. Good spatial and temporal resolution achievable.

7. Low leakage current. At room temperature the resistivity of diamond is about 6 orders of magnitude higher than that of silicon. Due to such high resistivity and large band gap the leakage current is extremely small.

Due to the aforementioned advantages of diamond-based detectors, they are used in several fields, such as high energy physics, medical therapy, synchrotrons and cyclotrons. Table 2.3 summarizes the main properties of silicon and diamond crystals. The signal generated in a diamond-based detector is faster than that generated in a silicon-based detector, because the carrier mobilities in diamond are larger than that in silicon. Furthermore, in diamond-based detectors, more energy is required to damage their crystal structure, since the displacement energy of diamond ( $43 \mathrm{eV} /$ atom $)$ is larger than that of silicon $(13-20 \mathrm{eV} /$ atom). Finally, it can be concluded, based on the average signal values for both diamond and silicon (table 2.3), that the signal amplitude for the same thickness detector is about half for diamond when compared to silicon. 
Table 2.3: Properties of silicon and diamond crvstals Ahmed, 2007: Breidenbach et al., 2004; Stolz, 2013].

\begin{tabular}{|l|l|l|}
\hline Property & Silicon & Diamond \\
\hline Band gap, $E_{g}(\mathrm{eV})$ & 1.12 & 5.5 \\
\hline Resistivity $(\Omega \mathrm{cm})$ & $2.3 \times 10^{5}$ & $>10^{11}$ \\
\hline Intrinsic carrier density $\left(\mathrm{cm}^{-3}\right)$ & $1.5 \times 10^{5}$ & $<10^{3}$ \\
\hline Electron mobility, $\mu_{e}\left(\mathrm{~cm}^{2} / \mathrm{Vs}\right)$ & 1500 & 1800 \\
\hline Hole mobility, $\mu_{h}\left(\mathrm{~cm}^{2} / \mathrm{Vs}\right)$ & 450 & 1200 \\
\hline e- $\mathrm{h}$ creation energy $(\mathrm{eV})$ & 3.6 & 13 \\
\hline Displacement energy $(\mathrm{eV} /$ atom $)$ & $13-20$ & 43 \\
\hline Mass density $\left(\mathrm{g} / \mathrm{cm}^{3}\right)$ & 2.33 & 3.52 \\
\hline Dielectric constant & 11.9 & 5.7 \\
\hline Radiation length, $X_{0}(\mathrm{~cm})$ & 9.4 & 12.2 \\
\hline Average signal $(\mathrm{e}-\mathrm{h} / \mu \mathrm{m})$ & 89 & 36 \\
\hline
\end{tabular}

\subsection{Instrumentation for non-destructive beam diagnostics}

\subsubsection{Transformers}

The beam current is the basic quantity of any charged particle beam. It is the first check of any accelerator functionality. In several cases, the beam current is measured with transformers. Their principle of operation is based on measuring the magnetic field induced by the beam (figure 2.11). Transformers are non-destructive devices i.e., they are independent of the beam energy. In an accelerator, the beam current of $N$ charged particles of charge state $q$ per unit time $t$ or length $l$ and velocity $\beta=v / c$ is given by equation 2.4 [Forck, 2011].

$$
I_{\text {beam }}=q e \cdot \frac{N}{t}=q e \cdot \beta c \cdot \frac{N}{l}
$$

where $e$ is the electron charge $\left(e=1.6 \times 10^{-19} C\right)$.

The magnetic field carried by the beam has only azimuthal component due to the cylindrical symmetry, as shown in figure 2.11. The relation between this 


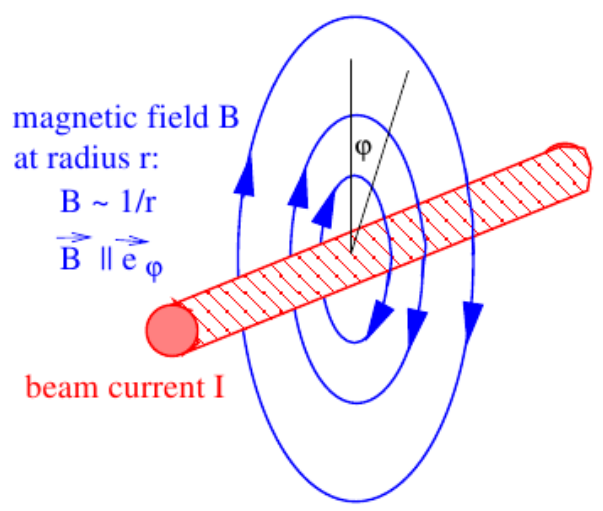

Figure 2.11: Schematic view showing the magnetic field induced by a charged particle beam [Forck, 2011].

Torus to guide the magnetic field

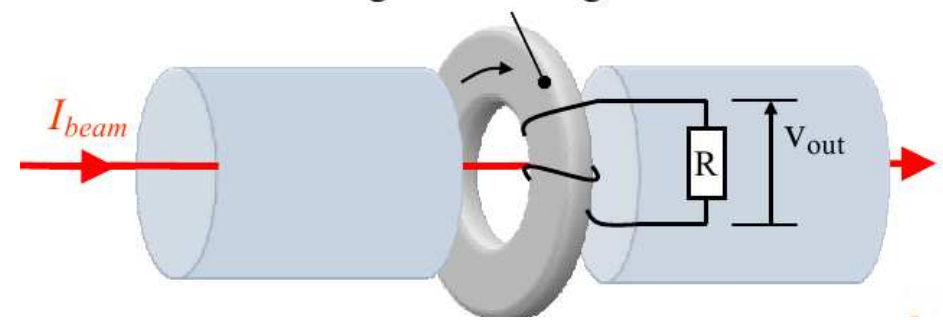

Figure 2.12: Schematic view of a current transformer for pulsed beams. The pulsed beam, such as a current-carrying wire, creates a magnetic field and the transformer feels this field.

magnetic field and the beam current and distance $r$ is given by equation 2.5 ,

$$
\vec{B}=\mu_{0} \frac{I_{b e a m}}{2 \pi r} \vec{e}_{\varphi}
$$

with $\mu_{0}=4 \pi \cdot 10^{-7}$ V.s $/ A$.m being the magnetic permeability of the vacuum and $r$ being the distance between the center of the beam and the point where the magnetic field is measured.

The pulsed beam current can be determined by monitoring the accompanied magnetic field using a current transformer (figure 2.12). In transformers, the pulsed beam is considered as a primary winding. An insulated wire wrapped around a torus with $N$ turns, serves as a secondary winding of the transformer with an inductance $L$. The torus is used in order to: (1) increase the inductance of the transformer; and (2) guide the magnetic field lines to the secondary winding, 
where only the azimuthal component of the magnetic field is measured. The inductance of a torus of relative permeability $\mu_{r}$ and $N$ windings is reported in equation 2.6 .

$$
L=\frac{\mu_{0} \mu_{r}}{2 \pi} \cdot l N^{2} \cdot \ln \left(\frac{r_{\text {out }}}{r_{\text {in }}}\right)
$$

where $l$ is the length of the torus material in the beam direction, $r_{i n}$ is the inner radius of the torus, whereas the outer radius is $r_{\text {out }}$.

For a loaded current transformer with low ohmic resistance $R$, the primary current (beam current) $I_{\text {prim }}$ is a function of the secondary current $I_{\text {sec }}$ in the isolated wire wrapped around the torus (equation 2.7) [LHC team, 2013].

$$
\frac{I_{\text {sec }}}{I_{\text {prim }}}=\frac{N_{\text {prim }}}{N_{\text {sec }}}
$$

The beam acts as primary winding with one turn $\left(N_{\text {prim }}=1\right)$, therefore the beam current is given by equation 2.8 .

$$
I_{\text {beam }}=N \cdot I_{\text {sec }}
$$

where $N$ being the number of turns in the secondary winding. For most practical cases a measurement of voltage $V$ is preferred, therefore the resistance $R$ is introduced (figure 2.12), leading to equation 2.9.

$$
V=R \cdot I_{\text {sec }} \Rightarrow I_{\text {beam }}=N \cdot \frac{V}{R}
$$

At LHC 1 two DC current transformers (DCCT) (figure 2.13) and two fast beam current transformers (FBCT) are being used per ring i.e., eight transformers in total are being used because the LHC has two rings. The principles of operation of the DCCT and the FBCT transformers are beyond the scope of this thesis.

\subsubsection{Secondary electron monitors}

The emission of secondary electrons from a metallic surface due to beam energy loss can be exploited in order to assess the intensity of high current beams. Such

\footnotetext{
${ }^{1}$ LHC stands for large hadron collider.
} 


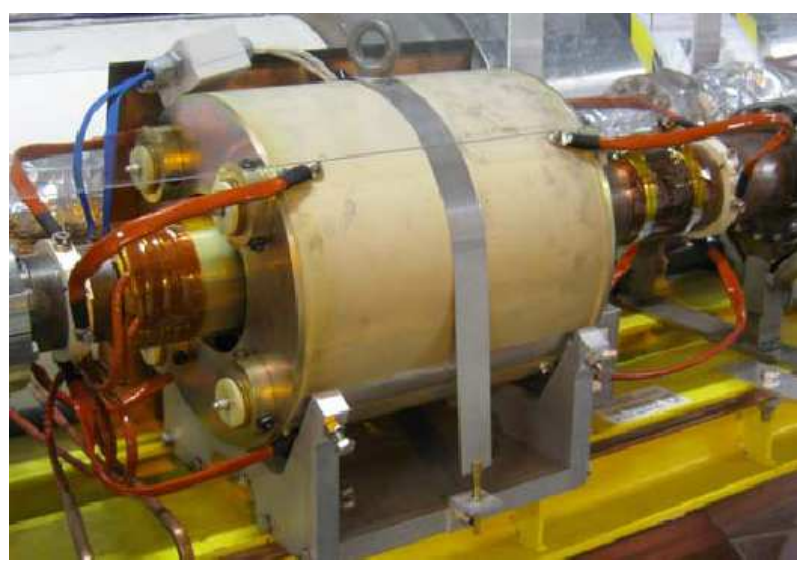

Figure 2.13: Photograph of DC current transformer (DCCT) being used at LHC at CERN [Odier et al., 2009].

a setup is shown schematically in figure 2.14, where three $100 \mu \mathrm{m}$ thick aluminum foils are used. The outer two foils are biased by typically $100 \mathrm{~V}$ to ensure that the liberated electrons are rapidly cleared away. Otherwise, an electron cloud may form over the foil surface and impede further emission. The middle foil is connected to a sensitive current amplifier where the beam current $I_{\text {beam }}$ can be assessed by measuring the secondary electron current $I_{s e c}$. The formula that relates both currents is known as Sternglass formula, which is reported in equation 2.10 Forck, 2011; Sternglass, 1957.

$$
I_{\text {sec }}=Y \cdot \frac{d E}{\rho d x} \cdot I_{\text {beam }}
$$

with $Y$ being the yield factor describing the amount of secondary emission per unit of energy loss at the surface of the metal foil, $\rho$ is the density of the foil material, and $d E / d x$ is the stopping power for the incident beam in this material. It must be taken into account that only electrons liberated close to the surface have enough energy to overcome the work function 1 of the metal. As a result, most of the liberated electrons are emitted with a relatively small kinetic energy of less than $10 \mathrm{eV}$ due to the thermalization process inside the material. Since this phenomenon is a surface phenomenon, the secondary emission coefficient is not only depend on the material but also on the state of cleanliness of its surface [Karzmark, 1964; Koziol, 2010].

\footnotetext{
${ }^{1}$ Work function $(\Phi)$ is the least energy necessary to free an electron from a metal surface. The work function of aluminum, for example, is $4.08 \mathrm{eV}$ [Tipler and Llewellyn, 2008]
} 


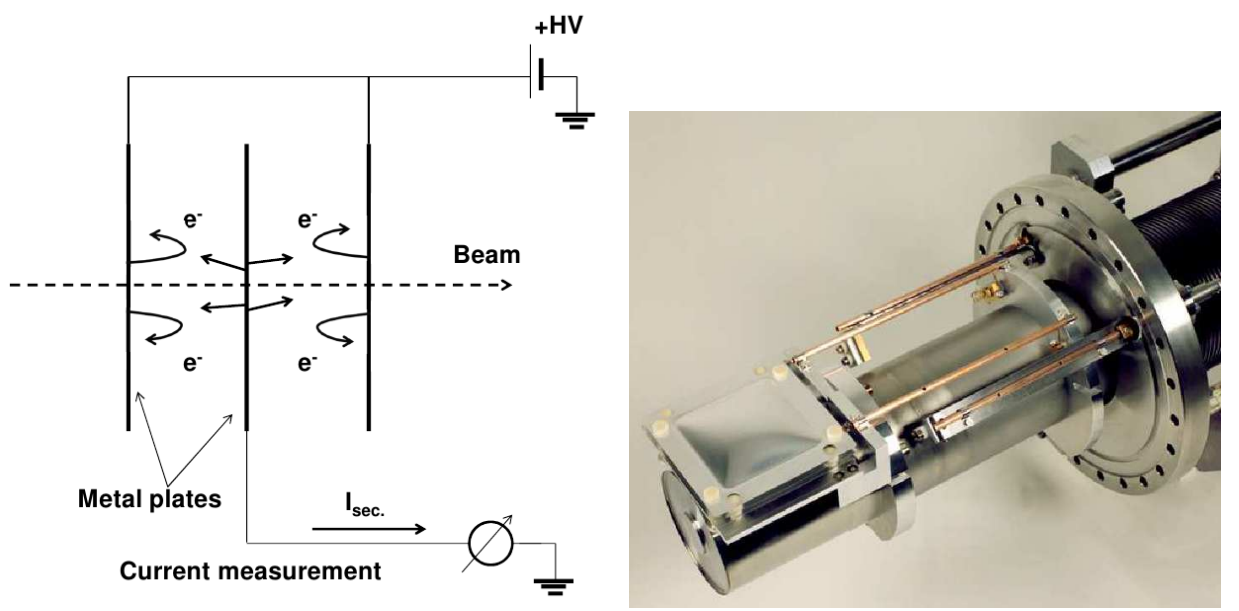

Figure 2.14: Schematic diagram (left) and photograph (right) of a secondary electron monitor made of 3 aluminum foils used for current measurements at the Gesellschaft für Schwerionenforschung (GSI) in Germany [Forck, 2011].

\subsubsection{Secondary electron emission (SEM) grids}

Secondary electron emission (SEM) grids make use of the phenomenon that under the impact of beam particles on some solid materials, electrons are liberated from their surfaces, therefore producing a flow of current. Beam position and beam intensity can be assessed using an array of sensing wires with typically $1 \mathrm{~mm}$ spacing (figure 2.15). The sensing wires pick up the beam current when the grid crosses the beam.

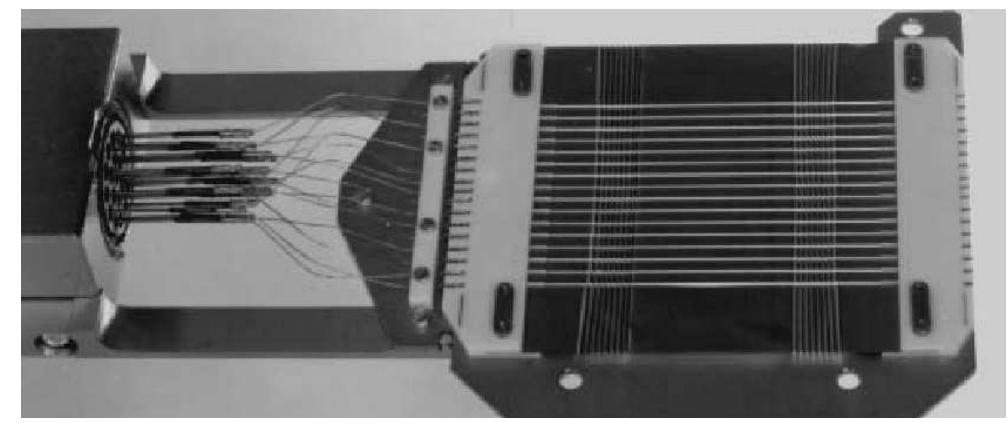

Figure 2.15: Photograph of a SEM grid made of thin ribbons attached to contacts on a ceramic frame [Koziol, 2010].

For assessing the beam profile, individual wires of a SEM grid interact with the beam; each wire has an individual current-to-voltage amplifier. W-Re (tungstenrhenium) alloys are often used in fabricating the metallic wires for their excellent refractory properties [Savitskii et al., 1960]. A SEM grid in the form of an array 
of wires is a much-used device in assessing beam profiles. Sequential display of the signals from the wires gives the beam profile as shown in figure 2.16. To enhance the signal strength, the whole array can be inclined with respect to the beam direction, therefore presenting a greater effective surface. When the signal strength is not a problem, the array and the electrodes can be made of thin wires, as a result this instruments becomes nearly a non-destructive beam profile monitor [Koziol, 2010].
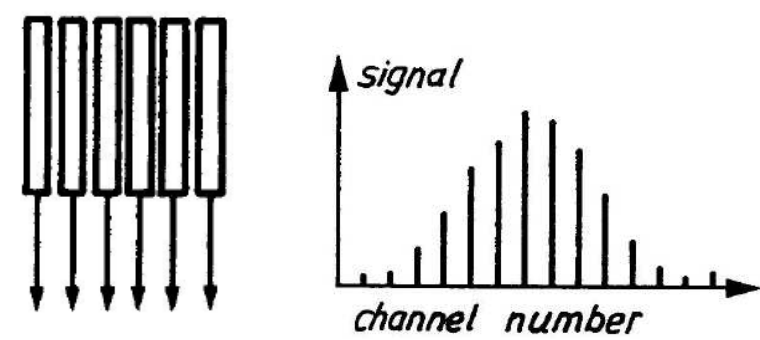

Figure 2.16: Scheme shows a SEM grid consisting of an array of sensing wires, seen in beam direction (left) and transverse beam profile obtained from the sensing wires (right).

Care has to be taken to prevent overheating by energy loss. For low energy beams, the ratio between wire spacing to the wire width can be 10 (spacing-todiameter ratio $=1 \mathrm{~mm} / 0.1 \mathrm{~mm}=10)$ i.e., the energy loss in this case represents only $10 \%$. On the other hand, for high energy beams $\left(E_{k i n}>1 \mathrm{GeV} / \mathrm{u}\right)$, wires with larger width can be used, due to negligible energy loss in this case [Forck, 2011.

Park et al. [2006] developed a current sensor module in order to measure the beam profile of a $45 \mathrm{MeV}$ proton beam from a cyclotron. The wires of the sensor were made from tungsten and they were assembled into an array and placed in parallel with the beam direction. The current output from each wire is amplified using a trans-resistance amplifier. When the wire ensemble is scanned across the beam, the beam position and intensity can be obtained from the measured output current of each wire. Normally, all sensing wires are placed in the perpendicular direction to the incident beam, as shown in figure 2.17, left. The wire in this configuration measures the average beam current along itself. In such case, it is difficult to obtain the beam position accurately. To overcome this problem. Park et al. [2006] proposed to place the sensor in parallel with the incident beam. In this case, each wire detects the beam intensity at the given position. Therefore, by scanning the array vertically, the whole beam profile can be determined accurately. The current from each wire is converted into a voltage by a cascade differential amplifier, then the measured voltage is digitized to determine the 
beam properties.

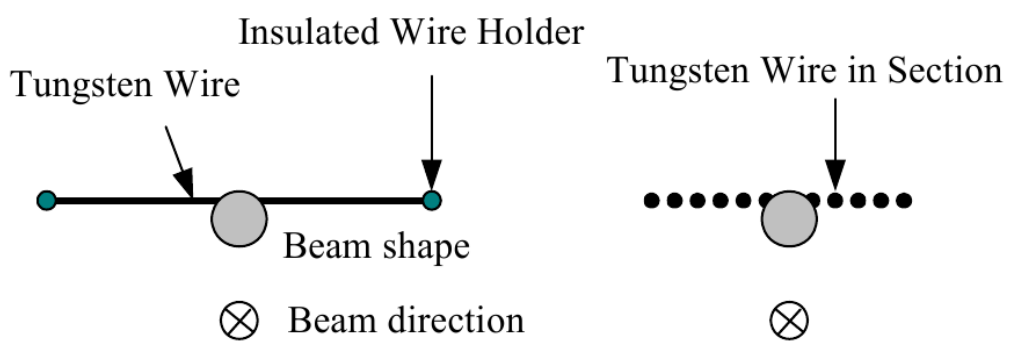

Figure 2.17: Schematic view shows a conventional BPM (beam position monitor) (left) and the one proposed by Park et al. [2006] (right). In the conventional BPM, all sensing wires are placed in the perpendicular direction to the incident beam, whereas in the proposed one all the wire are placed in a direction parallel to the beam.

\subsubsection{Wire scanners}

The SEM grid, which is made of several wires (with individual expensive electronics), disturbs the beam too much, mostly through multiple Coulomb scattering. Therefore, instead of using a fixed grid, one single wire can be used to scan the beam, as shown in figure 2.18. In wire scanners, the resolution is not limited by the wire spacing, as the case of SEM grids. Thus beam position and beam profiles can be measured with high spatial resolution [Forck, 2011].

A fast moving wire can be used even on a circulating beam. Speeds of $20 \mathrm{~m} / \mathrm{s}$ have been obtained with a thin carbon wire, which allowed beam profiles to be assessed without disturbing the beam [Koziol, 2010].

The wire is usually made from carbon due to its low weight and low nuclear charge. As a result, low energy loss occurs due to Bethe-Bloch formula $(d E / d x \propto$ $\rho \cdot Z / A)$. Furthermore, this material can withstand high temperatures without melting. The thickness of the wire can be down to $10 \mu \mathrm{m}$ in order to achieve high resolution.

\subsubsection{Gas-filled chambers}

Ionization chambers are one of the earliest constructed radiation detectors. Because of their simplicity in design and well understood physical processes, they are 


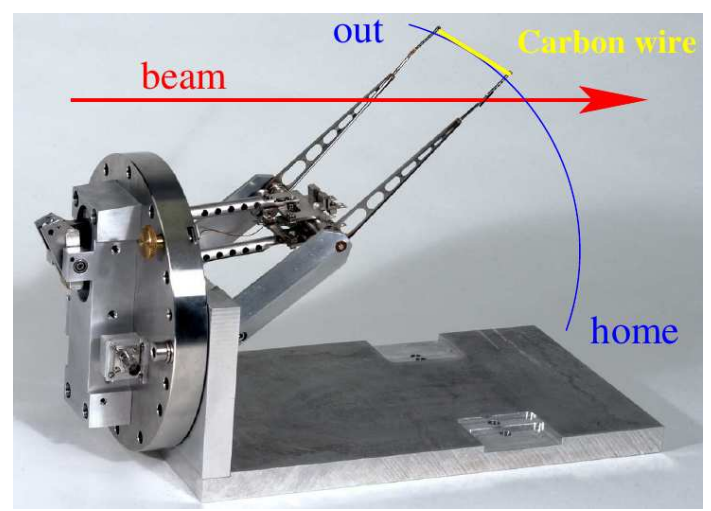

Figure 2.18: Pendulum scanner or "flying wire" being used at CERN synchrotron. The wire is extended between tips of two lightweight arms [Forck, 2011].

still one of the most widely used detectors. These devices are based on direct collection of electrons and ions created in an active gas by passing radiation through it. A gas is the most obvious medium to be used for collection of ionization from radiation, because of the high mobility of electrons and ions in this medium. The ionization chamber is composed basically from two parallel plates maintained at opposite electrical potentials, as shown in figure 2.19. The curvature in the electric lines of force at the edges of such detector can potentially cause nonlinearity in the response of the ionization chamber to the incident charged beams, however with proper designing this problem can be overcome. Figure 2.20 shows three metalized electrodes confined with active gas inside a chamber. Two of these metalized electrodes are biased with about $1 \mathrm{kV}$ to separate charges generated in the gas gaps. The third electrode measures the amount of secondary charge with a current amplifier [Forck, 2011; Leo, 1994].
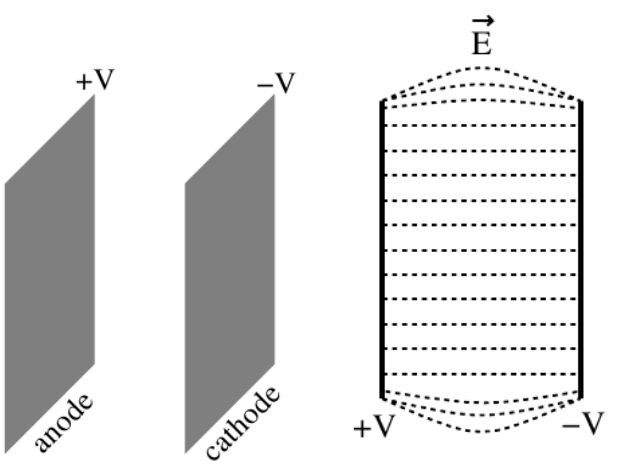

Figure 2.19: Schematic diagram of a parallel plate ionization chamber (left) and a two dimensional view of electric field inside its active volume (right). The curved electric field at the sides may induce nonlinearity in the response of the ionization chamber to incident beam [Ahmed, 2007]. 


\subsection{Instrumentation for non-destructive beam diagnostics}

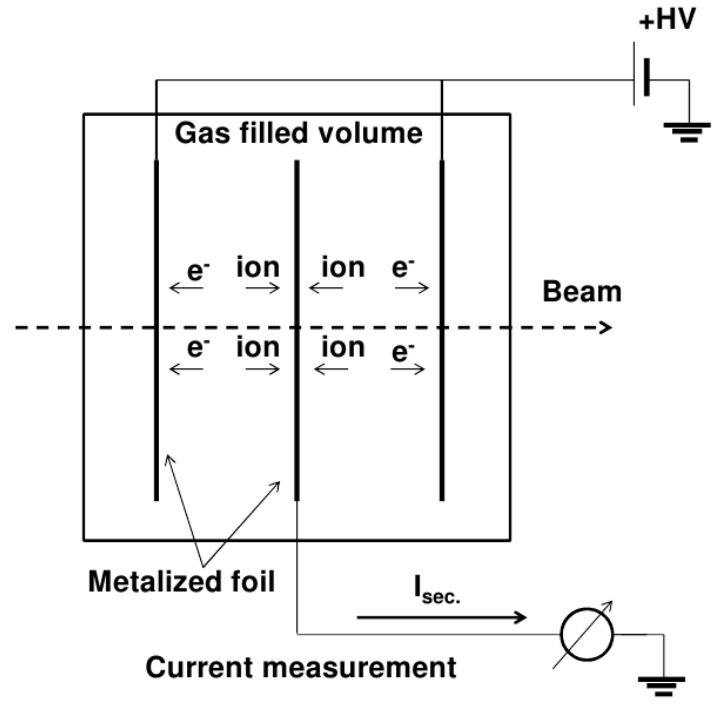

Figure 2.20: Diagram of ionization chamber. It shows three metalized electrodes confined with active gas inside the chamber. Charged particles passing through the chamber ionize the gas, the ions travel towards the cathode, whereas the electrons travel towards the anodes, thus the beam current can be measured [Forck, 2011].

When the beam crosses about $5 \mathrm{~mm}$ of active gas, the collisions with the gas molecules produce ion-electron pairs i.e., charged molecules and free electrons. Due to the biased voltage between the two electrodes of the ionization chamber, an electric field is present; therefore the ions and electrons move apart, each moving in opposite directions along the electric field lines until they reach the electrode conductors that are producing the electric field. The voltage should be in the plateau region (ion chamber region), where all generated charges are collected but no avalanche occurs (figure 2.21). The collection efficiency of electron-ion pairs in the recombination region increases with applied voltage until all created charges get collected. Further increasing the biased voltage does not affect the measured current. The current measured by the associated electronics in this region (i.e., ion chamber region) is called saturation current and is proportional to the energy deposited by the incident radiation.

Due to the large statistical fluctuations associated with the energy required to produce an electron-ion pair, its average value is used, which it is called the $\mathrm{W}$-value. This value represents all ionizations that occur inside the active volume of the chamber. Sometimes it is important to know the primary ionization yield as well. However, because of almost inevitable secondary ionizations that occur at nominal applied voltages, it is important to determine this number experi- 


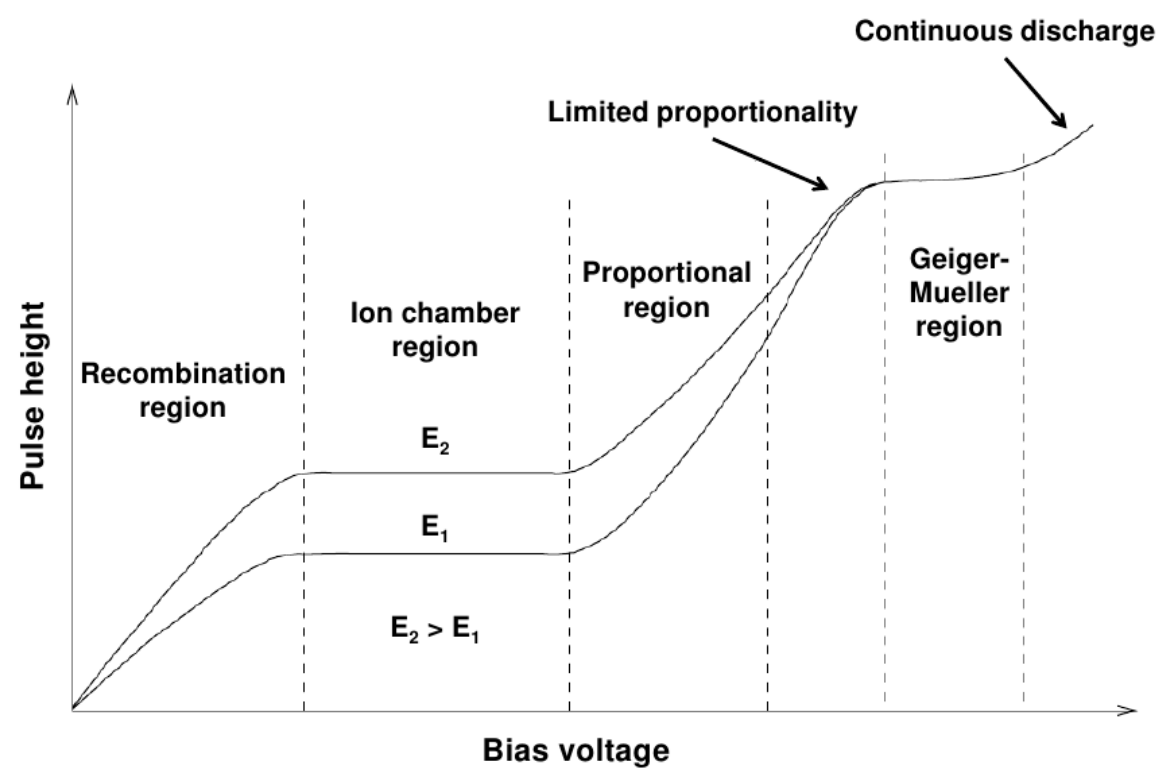

Figure 2.21: Variation of pulse height produced by different types of detectors with respect to applied voltage. The two curves correspond to two different energies of incident radiation. In case of ionization chambers, the biased voltage should be in the plateau region (ion chamber region), where all charges are collected but no avalanche occurs [Ahmed, 2007].

mentally. Table 2.4 summarizes W-value, primary ionization yield, and total ionization yield for some gases at standard atmospheric conditions for minimum ionizing particles.

In the ion chamber region, all electrons created by the beam in the active length $\Delta x$ of the ionization chamber are collected at the anode of the chamber. By using the $\mathrm{W}$-value of the gas and the calculated linear energy transfer of the beam in the gas, $d E / d x$, the beam current $I_{\text {beam }}$ can be calculated using equation 2.11. The precision of such measurements depends mainly on the accuracy of the stopping power calculations. A secondary current down to the $p A$ region can be measured precisely using ionization chambers [Yun et al., 2008].

$$
I_{\text {sec }}=\frac{1}{W} \cdot \frac{d E}{d x} \triangle x \cdot I_{\text {beam }}
$$

In most cases, pure argon $(A r)$ is used as an active gas in ionization chambers, sometimes it is mixed with about $10 \%$ of molecular gases such as $\mathrm{CO}_{2}$. Gases 
Table 2.4: Ionization potentials $I_{e}$, W-values, stopping powers $(d E / d x)$, primary ionization yield $n_{p}$, and total ionization yield $n_{t}$ for some gases ( $i p$ stands for the number of electron-ion pairs) Ahmed [2007]; Forck [2011].

\begin{tabular}{|c|c|c|c|c|c|c|}
\hline Gas & $\begin{array}{c}\text { Density } \\
\left(\times 10^{-4} \mathrm{~g} / \mathrm{cm}^{3}\right)\end{array}$ & $I_{e}(\mathrm{eV})$ & $\begin{array}{l}\text { W-value } \\
(\mathrm{eV} / \text { pair })\end{array}$ & $\begin{array}{l}d E / d x \\
(\mathrm{keV} / \mathrm{cm})\end{array}$ & $\begin{array}{l}n_{p} \\
(\mathrm{ip} / \mathrm{cm})\end{array}$ & $\begin{array}{c}n_{t} \\
(\mathrm{ip} / \mathrm{cm})\end{array}$ \\
\hline $\mathrm{H}_{2}$ & 0.8 & 15.4 & 37 & 0.34 & 5.2 & 9.2 \\
$\mathrm{He}$ & 1.6 & 24.6 & 41 & 0.32 & 5.9 & 7.8 \\
$\mathrm{~N}_{2}$ & 11.7 & 15.5 & 35 & 1.96 & 10 & 56 \\
$\mathrm{O}_{2}$ & 13.3 & 1.2 & 31 & 2.26 & 22 & 73 \\
$\mathrm{Ne}$ & 8.4 & 21.6 & 36 & 1.41 & 12 & 39 \\
$\mathrm{Ar}$ & 17.8 & 15.8 & 26 & 2.44 & 29 & 94 \\
$\mathrm{Kr}$ & 34.9 & 14.0 & 24 & 4.60 & 22 & 192 \\
$\mathrm{Xe}$ & 54.9 & 12.1 & 22 & 6.76 & 44 & 307 \\
$\mathrm{CO}_{2}$ & 18.6 & 13.7 & 33 & 3.01 & 34 & 91 \\
$\mathrm{CH}_{4}$ & 6.7 & 10.8 & 28 & 1.48 & 46 & 53 \\
\hline
\end{tabular}

with high electron affinity 1 , like $\mathrm{O}_{2}$ and $\mathrm{H}_{2} \mathrm{O}$, should be avoided. The presence of negative ions enhances the recombination with positive ions, resulting in a smaller amount of measured secondary charge.

Ionization chambers are not vulnerable to radiation because inert gases, single or diatomic, such as $A r$ and air are usually used in the detection volume of the chambers. Ionization chambers do not react as fast as scintillators, because the gas ions, created by the radiation, need about $100 \mu$ s to reach the electrodes. This time constant leads to a convolution of the primary signal [Forck, 2011].

Figure 2.22 shows schematically the layout of a free-air 2 ionization chamber. The collecting volume is defined as the column of air between the collecting electrode area and the high voltage electrode. Two guard electrodes are used to surround the collector with the same potential, although they do not contribute to the measured charge. They also serve to prevent leakage current from the high voltage electrode from reaching the collector [Ahmed, 2007; AIST team, 2009].

\footnotetext{
${ }^{1}$ The electron affinity describes the probability of electron capture.

${ }^{2}$ The $\mathrm{W}$-value for proton beams in dry air is $34.2 \mathrm{eV}$ [Jones, 2006].
} 


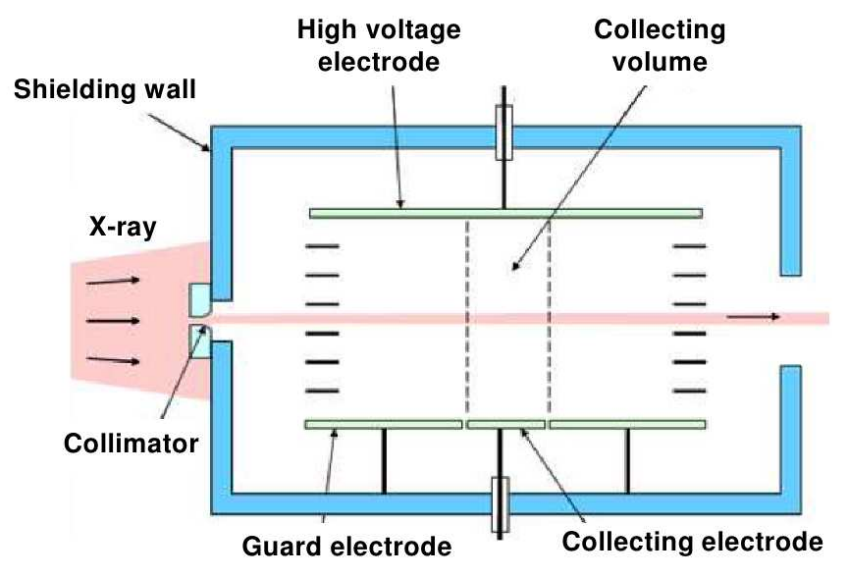

Figure 2.22: Schematic diagram of a parallel plate type free air ionization chamber. The small shaded volume element subtended by the narrow beam and the electric lines of force between anode and cathode form the measurement volume. The energy absorbed in this volume is actually measured and constitutes the absorbed dose. The guard electrodes are mainly used to smooth out the electric field non-linearities at the edges of the anode and also provide safety against high voltage on the anode [AIST team, 2009]. 


\section{Chapter 3}

\section{Proton beam monitoring system at PET-dedicated cyclotrons}

\subsection{The PET cyclotron at the University of Coim- bra}

Within the past three decades, the number of commercial cyclotrons for production of medical and industrial isotopes is increasing throughout the world. Different cyclotron models capable of accelerating protons up to $20 \mathrm{MeV}$ have been worldwide installed. The main goal of these cyclotrons is radionuclide production for positron emission tomography (PET). In addition, others aim in parallel at scientific experiments for production of medical and industrial isotopes, tumor therapy, applied studies using heavy ion beams (e.g., nanotechnology development), and nuclear waste transmutation [Papash and Alenitsky, 2008].

In Portugal, an IBA (Ion Beam Applications, Belgium) model Cyclone ${ }^{\circledR}$ 18/9 -HC (high current) cyclotron has been installed at ICNAS, University of Coimbra, for PET radionuclides. The cyclotron is located inside a bunker room, in a proper and specific infrastructure in underground floor (figure 3.1). The walls of the cyclotron bunker room are made of concrete with thickness of $200 \mathrm{~cm}$. The bunker room is closed during irradiation by a concrete door of the same thickness. The goal of this design is to limit the dose to the workers to $0.5 \mathrm{mSv} /$ year by shielding the neutron and gamma radiation produced by nuclear reactions during irradiation. 


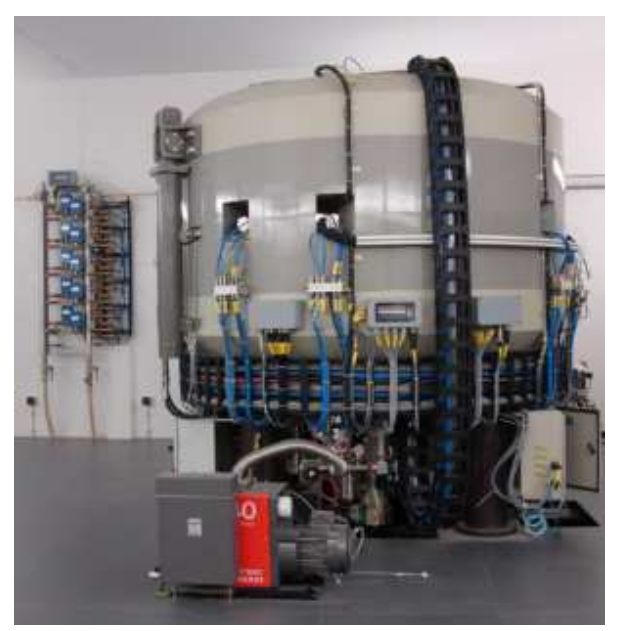

Figure 3.1: Photograph of the Cyclone ${ }^{\circledR}$ 18/9 -HC cyclotron inside the bunker room at ICNAS.

The control room of the cyclotron is located beside a power supply room, where a software application allows specialized personnel to check and control the radiofrequency $(\mathrm{RF})$ and vacuum inside the cyclotron, its magnets and ion source cabinets.

The production of the radionuclides is done by the irradiation of liquids, gases, and solids with $18 \mathrm{MeV}$ protons or $9 \mathrm{MeV}$ deuterons i.e., the radionuclides are produced by nuclear reactions. After that, the radionuclides are automaticaly and remotely transported to radiochemistry/radiopharmaceutical labs, where they are used in the syntheses of PET radiotracers and PET radiopharmaceuticals.

\subsubsection{Internal beam characteristics: Performance up to the beam port}

In the Cyclone ${ }^{\circledR}$ 18/9 -HC cyclotrons, negative ions $\left(\mathrm{H}^{-}\right.$ions) are produced at the center of the machine. These ions are subjected to a magnetic field and gain energy due to a high-voltage alternating electric field induced on two semicircular flat evacuated metallic Dees, where the ions are accelerated in spiral paths as shown in figure 3.2. When the ions reach nominal high energy (i.e., $18 \mathrm{MeV}$ ), they are extracted from the cyclotron by using charge exchange of $\mathrm{H}^{-}$ to $\mathrm{H}^{+}$on a thin carbon foil (5- $\mu$ m-thick) known as stripper foil, and therefore are bent out into primary beamlines. The negative ion cyclotron extraction process is called extraction by stripping (figure 3.2). The measured current on the 
stripper foil, which is located at the inner circumference of the magnet yoke of the PET cyclotron, is known as stripper current [Papash and Alenitsky, 2008]. Achievable ion beam currents on the stripper foils are 150 and $40 \mu \mathrm{A}$ for protons and deuterons, respectively [IBA team, 2013].

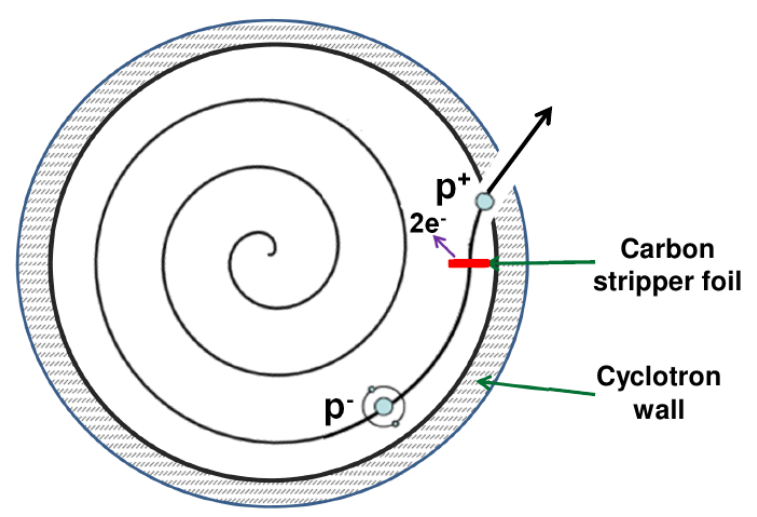

Figure 3.2: Schematic view representing extraction by stripping in a PET cyclotron. It shows the magnet yoke of the cyclotron and a stripper foil.

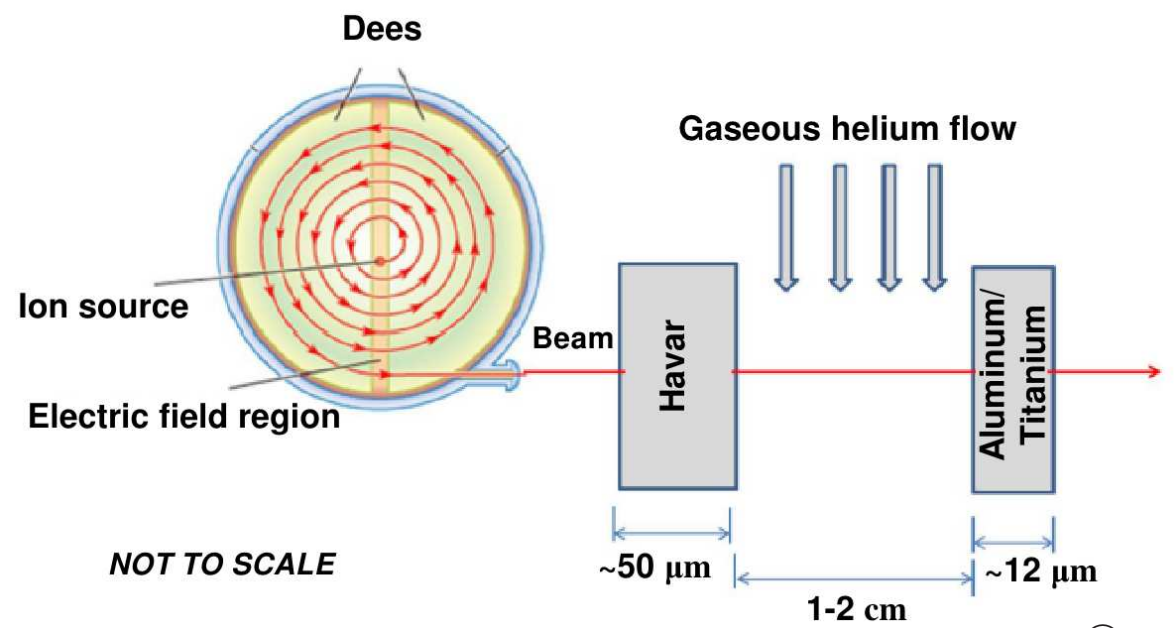

Figure 3.3: Schematic view of one of the exit ports of the Cyclone ${ }^{\circledR}$ 18/9-HC cyclotron. It shows a cyclotron, a $\operatorname{Havar}^{\circledR}$ foil, a gaseous helium flow, and an aluminum/titamium foil. The beam path is represented by a horizontal red arrow. The target for PET radionuclide production is not shown in the image.

The Cyclone ${ }^{\circledR}$ 18/9 -HC is manufactured by IBA since the early 1990 s as a commercial PET cyclotron. It is used for production of a whole range of medical isotopes. The cyclotron is raised above concrete floor to a height of $\sim 100 \mathrm{~cm}$ in order to place four diffusion pumps in addition to other equipment under the shell type magnet. Table 3.1 summarizes the main properties and parameters of the Cyclone $\left.{ }^{\circledR}\right)$ 18/9 -HC cyclotron. 


\section{Chapter 3. Proton beam monitoring system at PET-dedicated cyclotrons}

Table 3.1: Different parameters of the Cyclone ${ }^{\circledR}$ 18/9 -HC cyclotron IBA team, 2013; Papash and Alenitsky, 2008].

\begin{tabular}{|l|l|l|}
\hline Parameter & Unit & Value \\
\hline $\mathrm{H}^{-}\left(\mathrm{D}^{-}\right)$energy & $\mathrm{MeV}$ & $18(9)$ \\
\hline $\mathrm{H}^{-}\left(\mathrm{D}^{-}\right)$current & $\mu \mathrm{A}$ & $150(40)$ \\
\hline Energy variation & & $\mathrm{No}$ \\
\hline Average field & $\mathrm{kG}$ & 13.5 \\
\hline Magnet current & $\mathrm{A}$ & 200 \\
\hline Magnet power supply & $\mathrm{kW}$ & 24 \\
\hline Magnet weight & $\mathrm{t}$ & 20 \\
\hline RF $\mathrm{H}^{-}\left(\mathrm{D}^{-}\right)$frequency & $\mathrm{MHz}$ & $42(42)$ \\
\hline Number of Dees & & 2 \\
\hline RF harmonic H$\left(\mathrm{D}^{-}\right)$ & & $2(4)$ \\
\hline Voltage on Dee & $\mathrm{kV}$ & 32 \\
\hline Energy gain/turn & $\mathrm{keV}$ & $64(110)$ \\
\hline RF system power & $\mathrm{kW}$ & 10 \\
\hline Ion source & & 2 cold Penning ion gauge \\
\hline Current from the source & $\mathrm{mA}$ & $\sim 1.2$ \\
\hline Basic vacuum & Torr & $3 \times 10^{-6}$ \\
\hline Vacuum with the beam & Torr & $8 \times 10^{-6}$ \\
\hline Vacuum pumps & & 4 diffusion pumps \\
\hline$H^{-}$stripping losses & & $50 \%$ \\
\hline Number of extraction channels & & 8 \\
\hline Targets & & In yoke \\
\hline
\end{tabular}

The exit port of ICNAS cyclotron through which the ion beam is passed before leaving the cyclotron is composed of Havar ${ }^{\circledR} 1$ plus aluminum/titanium, with helium cooling in between as shown in figure 3.3 . The Havar ${ }^{\circledR}$ is a thin foil $(50 \mu \mathrm{m})$ used to maintain the necessary low operating pressure inside the cyclotron. In the outer end of the exit port usually a thin foil $(\sim 12 \mu \mathrm{m})$ of aluminum or titanium is placed in order to: (1) separate the gaseous helium from the exterior of the cyclotron; (2) support the target; and (3) act as a thermal bridge between the gaseous helium and the target [Pinto, 2010]. ICNAS cyclotron can accelerate protons and deuterons up to 18.2 and $9.2 \mathrm{MeV}$, respectively. Nevertheless, the energy of protons and deuterons after leaving one of the cyclotron exit ports is $17.5 \pm 0.2 \mathrm{MeV}$ and $8.2 \pm 0.1 \mathrm{MeV}$, respectively. This is due to energy loss in the exit port materials [Cunha, 2010].

\footnotetext{
${ }^{1}$ See appendix $\mathrm{A}$
} 


\subsubsection{Beam external to the cyclotron radius}

Proton and deuteron beams have nearly Gaussian distributions at targets for radiopharmaceutical production, which are located in air but still within the shielding of the cyclotron. Information provided by the manufacturer states that the FWHM (full width half maximum) at $30 \mathrm{~cm}$ after the exit port of the cyclotron is $10 \mathrm{~mm}$. According to the same source, this FWHM contains $80 \%$ of the protons and $70 \%$ of the deuterons. This beam dispersion is a result from the interaction of the beam with the exit port materials, mainly with the Havar ${ }^{\circledR}$ foil [Pinto, 2010]. For experimental procedures to be performed at setups outside the cyclotron radius, the beam path must be prolonged, which will change its characteristics.

\subsection{Characterizing external beam parameters}

In recent years, the interest of utilization of high energetic proton beams has been growing up not only in the fields of radiotherapy, but also in non-therapeutic applications such as radiation hardness tests of materials and devices for spacecraft, detector developments, and nuclear physics studies, among others [Kim et al., 2007; Papash and Alenitsky, 2008].

Proton beam current measurements are required to determine the dose delivered to a target. Dose rate and total dose can be monitored by ionization chambers 1, which are based on detecting direct ionization created by the passage of protons [Kim et al., 2006; Renner et al., 1989]. In addition to ionization chambers, the Faraday cup 2 is a widely used method for measuring proton beam current.

For proton irradiation experiments, the in-situ provision of information about the incident proton beam at user target is very important for developing technologies for beam utilization. Yun et al. [2008] developed a beam monitoring system to measure beam intensity and beam profile of an external proton beam entering a target with $45 \mathrm{MeV}$. The developed system composed of beam intensity monitor and beam profile monitor. An ionization chamber was chosen as a beam monitor for current measurements. The structure of the beam profile monitor is similar to that of the ionization chamber, except that the beam profile monitor is equipped with a position-sensitive electrode. Therefore, the proton beam profile

\footnotetext{
${ }^{1}$ See section 2.3 .5

${ }^{2}$ See section 2.2 .1
} 
can be assessed. Thereby, information on the proton beam can be assessed and monitored during irradiation.

A precise dosimetry is needed for successful radiobiology, radiophysiology, and dosimetric studies with proton beams from a PET-dedicated cyclotron. Several beam parameters have to be assessed in order to insure that the right value of dose is delivered to the target.

\subsubsection{Fluence}

In particle and accelerator physics, fluence is defined as the number of particles that enter an imaginary sphere, with a cross sectional area of $a\left[\mathrm{~m}^{2}\right]$, and it has units of $m^{-2}$ [Knoll, 2000]. It is considered one of the fundamental units in dosimetry, highly affected by beam divergence. A beam scattering system can deliver the desired beam fluence of protons on a target.

The accurate definitions of terms relating the number of accelerated particles $(N)$ are as the following:

Fluence, $\Phi$ : particles/unit area,

$$
\Phi=d N / d a
$$

Flux or intensity, $F$ : particles/unit time,

$$
F=d N / d t
$$

Flux density, $\phi$ : energy/unite area/unit time,

$$
\phi=d^{2} E / d t d a=E d \Phi / d t=E d F / d a
$$

where $E$ is the energy of the accelerated particles.

For our purpose of describing monoenergetic protons (i.e., $18 \mathrm{MeV}$ ), it is suitable to define, flux density, $\phi$ : particles/unit area/unite time,

$$
\phi=d^{2} N / d t d a=d \Phi / d t=d F / d a
$$




\subsection{Characterizing external beam parameters}

The beam current $I$ is defined as follows:

$$
I=q e \cdot F=q e \cdot d N / d t
$$

where $q$ being the charge state of the accelerated particles and $e$ is the elementary charge.

\subsubsection{Beam energy}

ICNAS facility is a computer controlled cyclotron with fixed energy of $18 \mathrm{MeV}$ for protons. Nevertheless, the energy of the protons after leaving one of the exit ports of the cyclotron is $17.5 \pm 0.2 \mathrm{MeV}$ [Cunha, 2010]. The energy measurement of proton beam with high accuracy is very important for many experiments, potentially including radiobiological and dosimetric studies. To adjust the energy level of a proton beam, two methods are generally used: (1) adjust the beam energy directly in the accelerator; and (2) adjust the beam energy after extraction from the accelerator. Since ICNAS cyclotron is a fixed energy facility, thus its beam energy can be adjusted after beam extraction only. A degrader system can be used to adjust the energy level of the beam used for various application fields. The energy degrading system can offer the desired beam energy. This system can be made of foils such as aluminum foils with different thicknesses.

\subsubsection{Bragg peak}

Protons have an energy curve with a very specific peak known as the Bragg peak (figure 3.4), where they deposit most of their energy. The Bragg peak can be measured using a scintillator readout by a CCD camera, for example. The distance between the points of $100 \%$ and $20 \%$ of post the Bragg peak is called post peak fall-off.

\subsubsection{Beam range}

The range of protons in a medium is defined as the depth of penetration measured along straight line parallel to the original direction of motion of protons from the point where they penetrate the medium to the point where they stop in it. From the Bragg peak curve (figure 3.4), the range is related to the distance between 
the entrance surface of the protons and the distal point of $50 \%$ of the Bragg peak as shown in figure 3.4 .

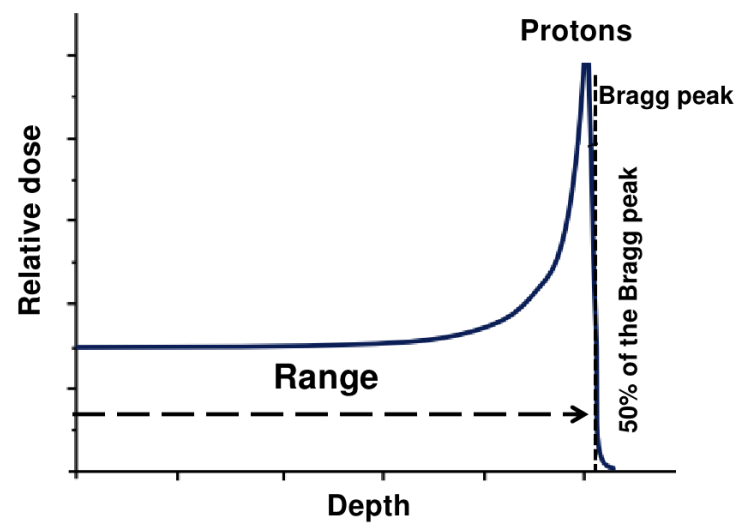

Figure 3.4: Depth-dose profile for proton beam in a medium. It illustrates the definition of the proton beam range in the medium.

\subsubsection{Beam current}

The proton beam current achievable on a stripper foil 11 at ICNAS cyclotron is $150 \mu \mathrm{A}$. However, it is possible to lower this value down to $\sim 1 \mu \mathrm{A}$ or even less by controlling the current on the ion source, $I_{\text {source }}$, using the computer system of the cyclotron. Several instruments can be used for measuring proton beam current, which might be destructive or non-destructive to the proton beam such as secondary electron monitors 2 .

\subsubsection{Beam profile}

The motivation for measuring beam profiles of proton beams is to know the spatial fluence distribution as well as the uniformity and the size of the beam spots at irradiated targets. Furthermore, extracted information from the beam profiles can be utilized in configuration of the beam tuning conditions. The proton beam from ICNAS cyclotron has nearly Gaussian distribution with a FWHM of $10 \mathrm{~mm}$ at $30 \mathrm{~cm}$ after the exit port, with $80 \%$ of the protons [Pinto, 2010]. The

\footnotetext{
${ }^{1}$ Stripper foil is a 5 - $\mu$ m-thick carbon foil. The measured current on this foil, which is located at the inner circumference of the magnet yoke of the PET cyclotron, is known as stripper current Papash and Alenitsky, 2008].

${ }^{2}$ See section 2.3.2.
} 


\subsection{Characterizing external beam parameters}

dispersion of the beam is a result from the interaction of the protons with the exit port materials, mainly with the Havar ${ }^{\circledR}$ window. Proton beam profiles can be assessed by several methods, such as dosimetric films (e.g., Gafchromic ${ }^{\circledR}$ EBT2) or plastic scintillators readout by CCD cameras. The proton beam is made to pass through a set of collimators before irradiating a target. Therefore, the collimators are the ones that define the shape of the beam.

\subsubsection{Dose and dose rate}

Radiation is capable of causing damage to both living and non-living things by energy deposition. If a high level of dose is delivered in a short period of time, the damage can be acute because the dose delivered per unit time is so high that the material does not get enough time to repair the damage. The acute damage depends on the time integrated energy deposited by the radiation. If the energy deposition time is shorter than the repair mechanisms, acute damage can occur. Therefore, it is important to use dose and dose rate to refer to the integrated energy deposited and the energy deposited per unit time, respectively.

The amount of energy deposited in a medium per unit mass of the medium by ionizing radiation is called absorbed dose. It is measured in units of gray i.e, $1 G y=1 \mathrm{~J} / \mathrm{kg}$. However, the same absorbed dose in some media are characterized by large damage in respect to other living media exposed to the same dose. In addition, absorbed dose treats all types of radiation equally i.e., there is no difference between a photon and a proton if they deposit the same amount of energy. Therefore, a quantity called equivalent dose has been defined that characterizes the damaging effect of radiation on tissues. The unit for equivalent dose is the sievert (Sv), which is one joule/kilogram. The equivalent dose $H_{T, R}$ (equation 3.6) can be obtained by multiplying the absorbed dose $D_{T, R}$ generated by the radiation type $R$ and the radiation weighting factor $W_{R}$. This factor is suited to the biological effectiveness of the particular type of radiation 1 . For photon and electron radiation, the radiation weighting factor $W_{R}$ has the value of one independently of the energy of the radiation, for other particle species see table 3.2 [Ahmed, 2007; European Nuclear Society, 2013; Knoll, 2000].

$$
H_{T, R}=W_{R} \cdot D_{T, R}
$$

The equivalent dose can be used for one tissue type only. To account for

\footnotetext{
${ }^{1}$ See section 1.2 .2 .2
} 
Table 3.2: Radiation weighting factor $W_{T}$ for different types of particles Ahmed, 2007; European Nuclear Society, 2013.

\begin{tabular}{|l|c|}
\hline Type of radiation & Radiation weighting factor, $W_{R}$ \\
\hline Muons & 1 \\
Alpha particles & 20 \\
Heavy nuclei & 20 \\
Protons $>2 \mathrm{MeV}$ & 5 \\
Neutrons & $5-20$ \\
\hline
\end{tabular}

Table 3.3: Tissue weighting factors $W_{T}$ according to 1990 recommendations of ICRP [Ahmed, 2007; European Nuclear Society, 2013].

\begin{tabular}{|l|c|}
\hline Tissue or Organ & Tissue weighting factor, $W_{T}$ \\
\hline Gonads & 0.20 \\
Bone marrow (red), Colon, Lung, Stomach & 0.12 \\
Bladder, Breast, Liver, Oesophagus, Thyroid & 0.05 \\
Skin, Bone surface & 0.01 \\
Pancreas, small intestine, uterus, & 0.05 \\
brain, spleen, muscle, suprarenal gland, & \\
kidney, thymus gland & \\
\hline
\end{tabular}

the differing radiosensitivity of various types of tissue, a set of tissue weighting factors $W_{T}$ (table 3.3) are introduced to allow calculation of what is now called the effective dose $E(\mathrm{~Sv})$, which is defined as reported in equation 3.7 .

$$
E=W_{T} \cdot H_{T}
$$

where $H_{T}$ is the equivalent dose in the tissue $T$.

In experiments of biological science, the measurement of dose, dose rate, and depth-dose distribution are very important. In general, dosimetric films and ionization chambers are the main methods used for measuring these quantities. Beam divergence dictates achievable doses and dose rates on targets. 


\subsection{Preliminary characterization of the external proton beam from ICNAS cyclotron}

\subsubsection{Motivation}

A cyclotron facility has been recently installed at ICNAS for production of radioisotopes for medical use. This facility is equipped with eight beamlines suitable for scientific research. Development of a proton beam monitoring system will be very useful for a number of different future developments and applications of proton beams. Such system is predicted to be capable of providing input for experiments in the fields of medical and biological sciences as well as in other scientific fields, such as materials radiation hardness tests for space applications. With this goal set for the long run, among others, in the present section experiments and simulation address the characterization of the proton beam achievable outside the yoke of the PET cyclotron from the University of Coimbra.

\subsubsection{Out-of-yoke proton beam}

For experimental procedures to be performed at setups outside the cyclotron radius, the beam path must be prolonged, which will change its characteristics. The common exit port windows at ICNAS cyclotron are made of Havar ${ }^{\circledR}$ plus titanium/aluminum foils and a flow of helium for cooling placed in between (figure 3.3). This can certainly be a major beam dispersing and energy degradation factor located inside the cyclotron. On the other end from the exit port to the outside wall of the cyclotron there are $40 \mathrm{~cm}$. In order to reduce the dispersive in-to-outside window and to transport the proton beam outside the cyclotron yoke, a 40-cm-long aluminum pipe (figure 3.5) was constructed and utilized. A vacuum isolation window (Havar ${ }^{\circledR}$ window) was fixed at the outer end of the aluminum pipe. This system allows the beam to travel in vacuum along these $40 \mathrm{~cm}$, bringing the exit port therefore outside the cyclotron shielding.

For a first trial of the divergence of the proton beam off the ICNAS cyclotron, the 40-cm-long aluminum pipe and a simple $50 \mathrm{~mm}$ CF (ConFlat) flange fitted with an extra O-ring were used. The O-ring was used to hold a 50- $\mu$ m-thick Havar ${ }^{\circledR}$ window $(30 \mathrm{~mm}$ in diameter). An aluminum disk was used to hold the Havar ${ }^{\circledR}$ window screwed to the CF flange, as shown in figure 3.5. The 40-cm-long aluminum pipe with the Havar ${ }^{\circledR}$ window was fixed to one of the exit ports of 


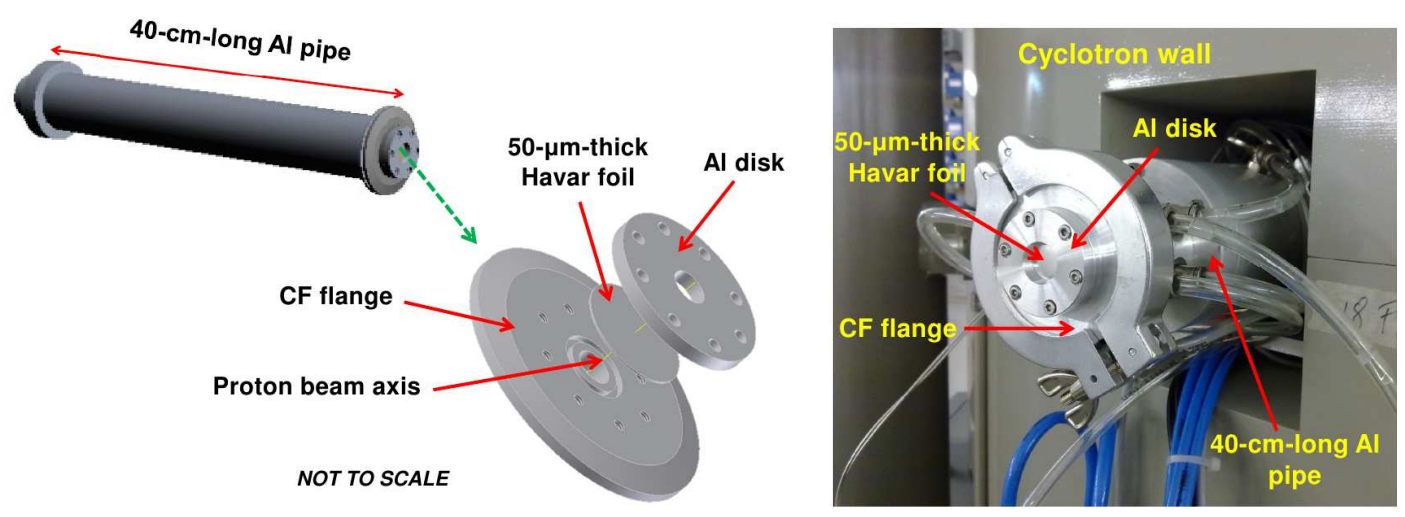

Figure 3.5: Schematic diagram (left) and photograph (right) of the 40-cm-long aluminum pipe. They show a 40-cm-long aluminum pipe, a CF (ConFlat) flange, a 50 - $\mu m$-thick $\operatorname{Havar}^{\circledR}$ window, and an aluminum disk.

ICNAS cyclotron, where the vacuum inside the aluminum pipe was adapted to be same as the vacuum inside the cyclotron $\left(\sim 10^{-5} \mathrm{mbar}\right)$. Therefore, the proton beam travels in the cyclotron vacuum inside the aluminum pipe and only after traversing the Havar ${ }^{\circledR}$ window it travels in air. This system allows to position experimental setups as close as possible to the exit gate of the cyclotron. In order to obtain first information about beam divergence and beam uniformity in this configuration, the experiments described in the next subsection were performed.

\subsubsection{Setup for the characterization of in-air beam diver- gence}

Several experiments were carried out at ICNAS cyclotron in order to provide a first insight onto the achievable in-air proton beam i.e., a beam contained within the concrete bunker walls but transported outside the cyclotron yoke. In addition, the Geant4 simulation toolkit [Agostinelli et al., 2003; Allison et al., 2006] (Geant4 version 9.3.p01) with the QGSP_BERT_HP physics package activated was utilized, after validation with published experimental data with protons of similar energy 1 Verbinski and Burrus, 1969], in order to explain experimental results described in this chapter. Furthermore, Geant4-based Monte Carlo was utilized in order to evaluate the possibility of using one beamline of ICNAS cyclotron for an out-of-yoke irradiation setup. Dose and dose rate on target were studied, together with the undesirable effects of neutron and $\gamma$-ray dose, the latter being produced in the cyclotron material, the irradiation setup, and the concrete

\footnotetext{
${ }^{1}$ See appendix B
} 

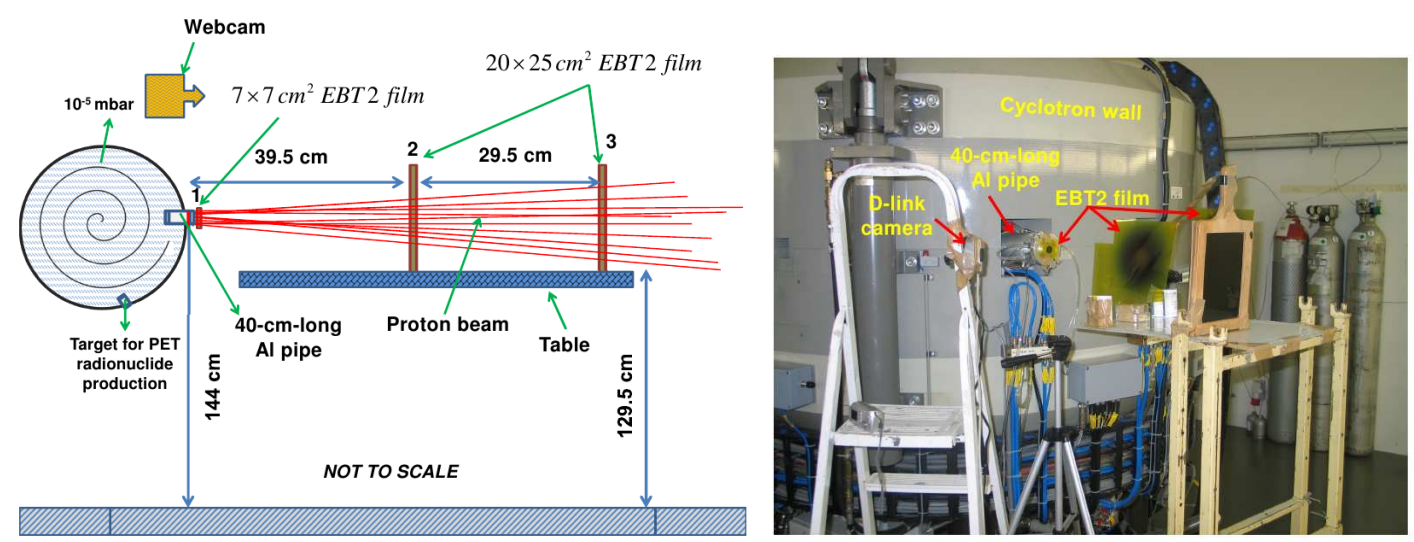

Figure 3.6: Experimental setup (left) and photograph (right) at ICNAS for measuring in-air proton beam divergence. Three films were placed at the outer side of the Havar ${ }^{\circledR}$ window, at a distance of $1,39.5$, and $69 \mathrm{~cm}$ from that window, respectively.

bunker walls.

\subsubsection{Experiment at the PET cyclotron at ICNAS}

The setup shown in figure 3.6 was utilized in order to provide a first measurement of the proton beam divergence in-air. The beam was transported outside the cyclotron yoke by means of an aluminum pipe specially built for that purpose (figure 3.5). A 50- $\mu$ m-thick Havar ${ }^{\circledR}$ window separated the vacuum inside the aluminum pipe from the air in the bunker. Three Gafchromic ${ }^{\mathbb{R}}$ external beam therapy (EBT2) film sheets were positioned perpendicularly to the beam axis at different distances in front of the exit port of the cyclotron.
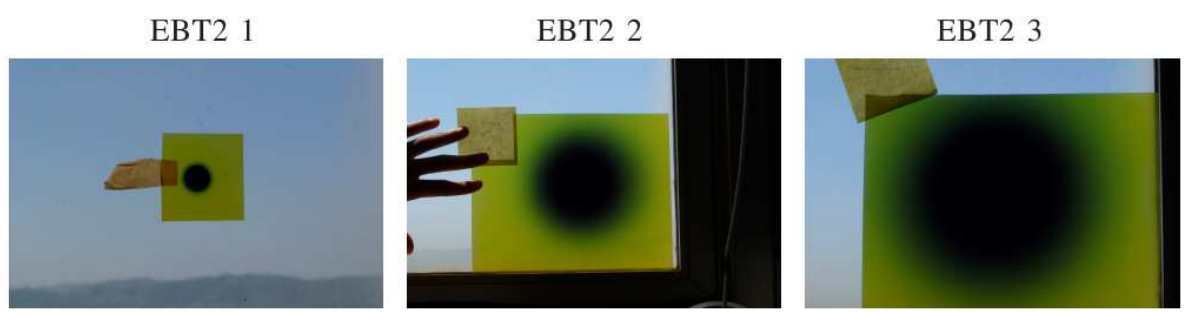

Figure 3.7: Experimental results obtained with three EBT2 Gafchromatic ${ }^{\circledR}$ films irradiated with the proton beam at ICNAS, with the setup of figure 3.6.

The color of the irradiated area in each film changed to black when exposed to ionizing radiation (i.e., proton beam) as shown in figure 3.7. This figure 


\section{Chapter 3. Proton beam monitoring system at PET-dedicated cyclotrons}

shows that the beam profile becomes broader with increasing distance in-air after the exit port of the cyclotron because of multiple scattering, mainly within the Havar ${ }^{\circledR}$ window.

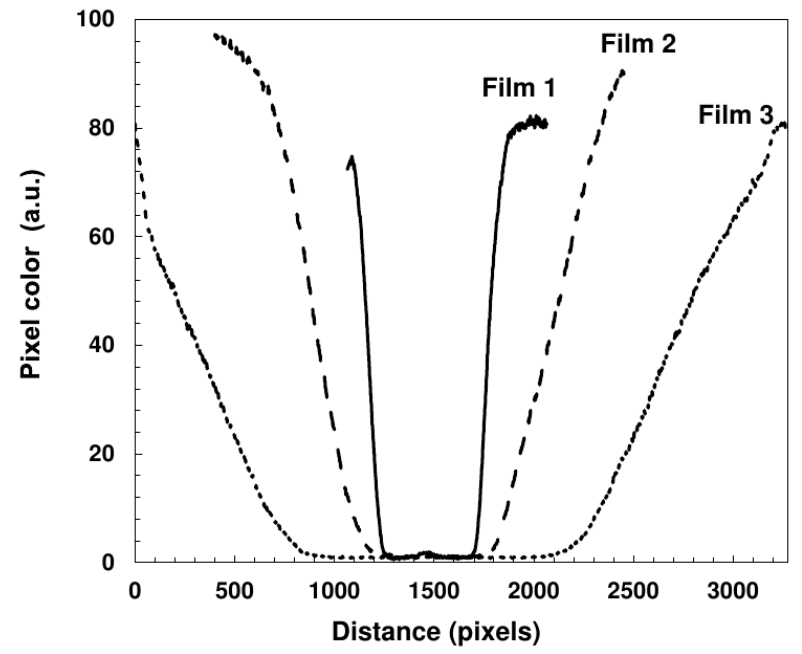

Figure 3.8: In-air beam profile obtained with three Gafchromic ${ }^{\circledR}$ EBT2 films (figure 3.7) irradiated with protons at ICNAS.

The normalized beam profiles of the three films are shown in figure 3.8. These profiles were obtained by scanning each film with a transparency scanner and further processing of the resulting image with the software package ImageJ [ImageJ team, 2004]. From the photographs and beam profiles (figures 3.7 and 3.8) of the three films, three regions can be distinguished, corresponding to film saturation (black), film proportional response (grey), and no-response (yellow) (figure 3.9). The first one is the saturated region (flat central interval region in the beam profiles). The second region is the proportionality region, which is located around the saturated region. The third outer region corresponds to the area where the irradiation was insufficient to impress the films.

The measured proton beam current 11 directly after the ion source of the cyclotron during irradiation was on average $2.7 \mu \mathrm{A}$, however during acceleration it is known that approximately half of the beam is lost. Thus the beam current outside the cyclotron was assessed to be $\sim 1.35 \mu \mathrm{A}$ i.e., the cyclotron delivered high density ion pulses at approximately $10^{13}$ protons/s. Figure 3.10 shows schematically the irradiated areas of the three EBT2 films used in the experiment (figure 3.6), where the irradiated area of the three films has nearly a circular shape with a diameter of 2.2, 18 and $25 \mathrm{~cm}$, respectively. In addition, it shows the divergence

\footnotetext{
${ }^{1}$ The beam current inside the cyclotron, directly after the ion source, is measured using a Faraday cup.
} 


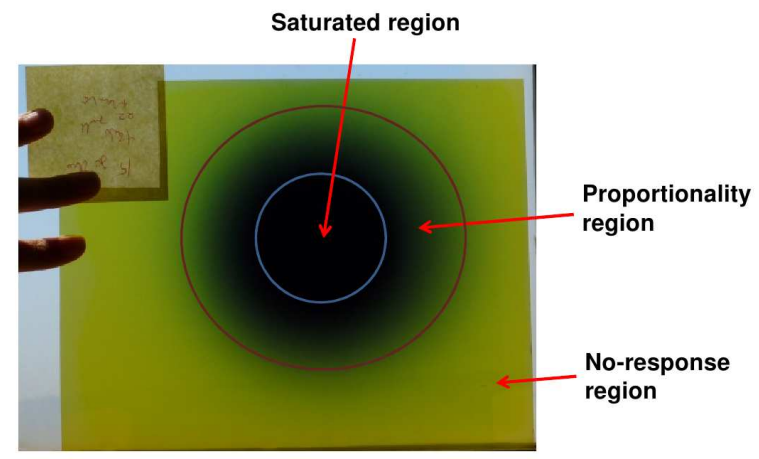

Figure 3.9: Saturated, proportionality, and no-response regions for the second Gafchromic ${ }^{\circledR}$ EBT2 film irradiated with protons from ICNAS cyclotron.

of the proton beam, where the angular width of the proton beam was found to be approximately $0.151 \mathrm{rad}\left(8.59^{\circ}\right)$.

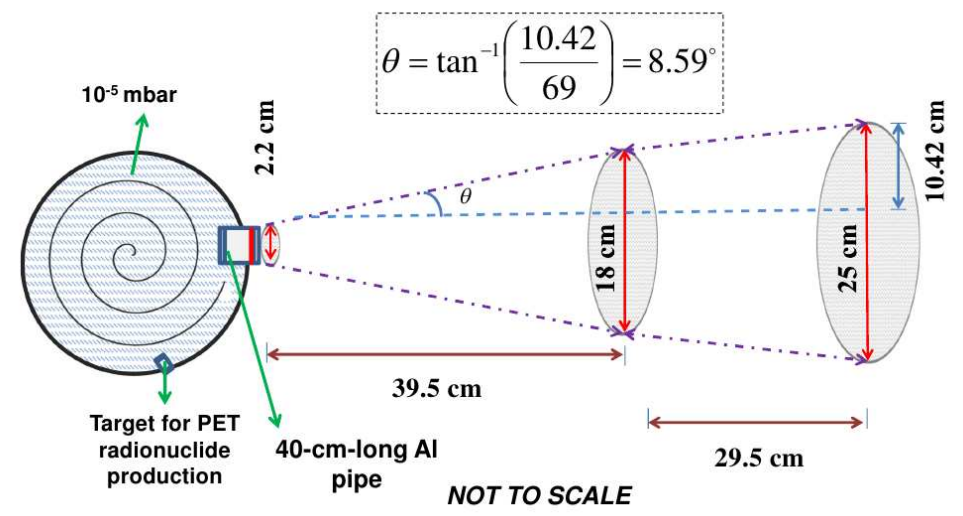

Figure 3.10: Schematic diagram of the irradiated area for the three EBT2 films irradiated with the proton beam at ICNAS (figure 3.6). It shows the divergence of the proton beam after one of the exit ports of ICNAS cyclotron.

The flux density $\phi$ at the position of each film was calculated using equation 3.8 .

$$
\phi\left[\text { protons } / \mathrm{s} \cdot \mathrm{cm}^{2}\right]=\frac{10^{13}[\text { protons } / \mathrm{s}]}{\pi r^{2}\left[\mathrm{~cm}^{2}\right]}
$$

where $r 1$ is the radius of the irradiated area, the values of the flux density at the locations of the three films are listed in table 3.4.

\footnotetext{
${ }^{1}$ The radius $r$ being taken as the full width at half maximum of the profiles shown in figure 3.8
} 


\section{Chapter 3. Proton beam monitoring system at PET-dedicated cyclotrons}

Table 3.4: The flux density values $\phi$ at the locations of the three EBT2 films that have been irradiated with protons at ICNAS.

\begin{tabular}{ccc}
\hline Film no. & $\begin{array}{c}\text { Distance from exit port } \\
(\mathbf{c m})\end{array}$ & $\begin{array}{c}\text { Flux density } \\
\left.\text { (protons } / \mathbf{s} \cdot \mathbf{c m}^{2}\right)\end{array}$ \\
\hline 1 & 1 & $2.6 \times 10^{12}$ \\
2 & 39.5 & $3.9 \times 10^{10}$ \\
3 & 69 & $2.0 \times 10^{10}$ \\
\hline
\end{tabular}

\subsubsection{Geant4 simulations}

The experimental setup of the EBT2 films experiment (figure 3.6) was transported into Geant4 allowing a simulation to be carried out in similar experimental conditions (figure 3.11). A proton beam (dark blue) is started inside the cyclotron and shot through the Havar ${ }^{\circledR}$ window (white circle), crossing three EBT2 films (cyan squares) positioned in air. Green trajectories correspond to neutral particles (neutrons or $\gamma$-rays), whereas red trajectories correspond to negative particles i.e., electrons arising from ionization in air or in the crossed material such as the $\operatorname{Havar}^{\circledR}$ foil.

The simulation results are shown in figure 3.12. It can be seen that the beam size observed in films 2 and 3 increases with distance for $100 \mathrm{k}, 1 \mathrm{M}$, and $10 \mathrm{M}$ initial protons. These results are consistent with the experimental ones. In addition, figure 3.12 shows that the beam size increases with increasing the number of protons started. This is expected, because by increasing the number of protons shot onto the EBT2 films, more protons deposit energy in the films, resulting in an increase of the radius of the saturated region. The beam size of the films in the first column does not increase with increasing the number of protons because this film was positioned at the outer surface of the Havar ${ }^{\circledR}$ window. There, the beam impresses the film with its initial beam radius of $7.5 \mathrm{~mm}$ before spreading itself due to beam divergence. This beam size represents the beam size inside the aluminum pipe that constitutes the beam port. Furthermore, it can be noticed that the dose increases with increasing the number of protons due to the increasing of the energy deposited in the films. Finally, the arrows in figure 3.12 show two regions of equal dose (cf. different dose color scales) with increasing radiuses due to the increasing total number of protons shot on film in each situation. 

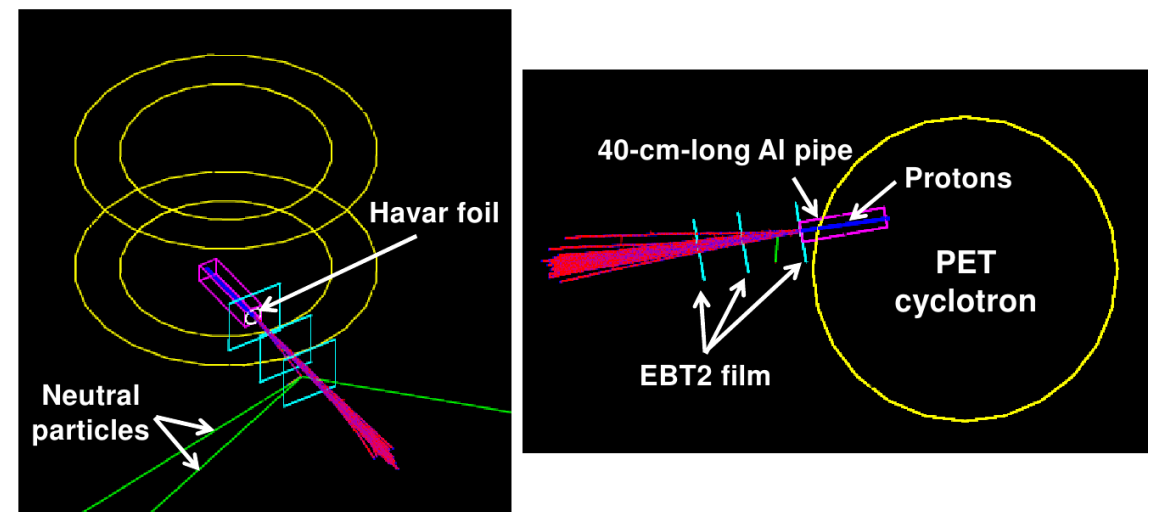

Figure 3.11: Two views (side view (left) and top view (right)) of the experimental setup of the EBT2 films experiment (figure 3.6) simulated with Geant4. Three EBT2 film (cyan) were positioned in-air and irradiated with protons(dark blue) from ICNAS cyclotron (yellow).

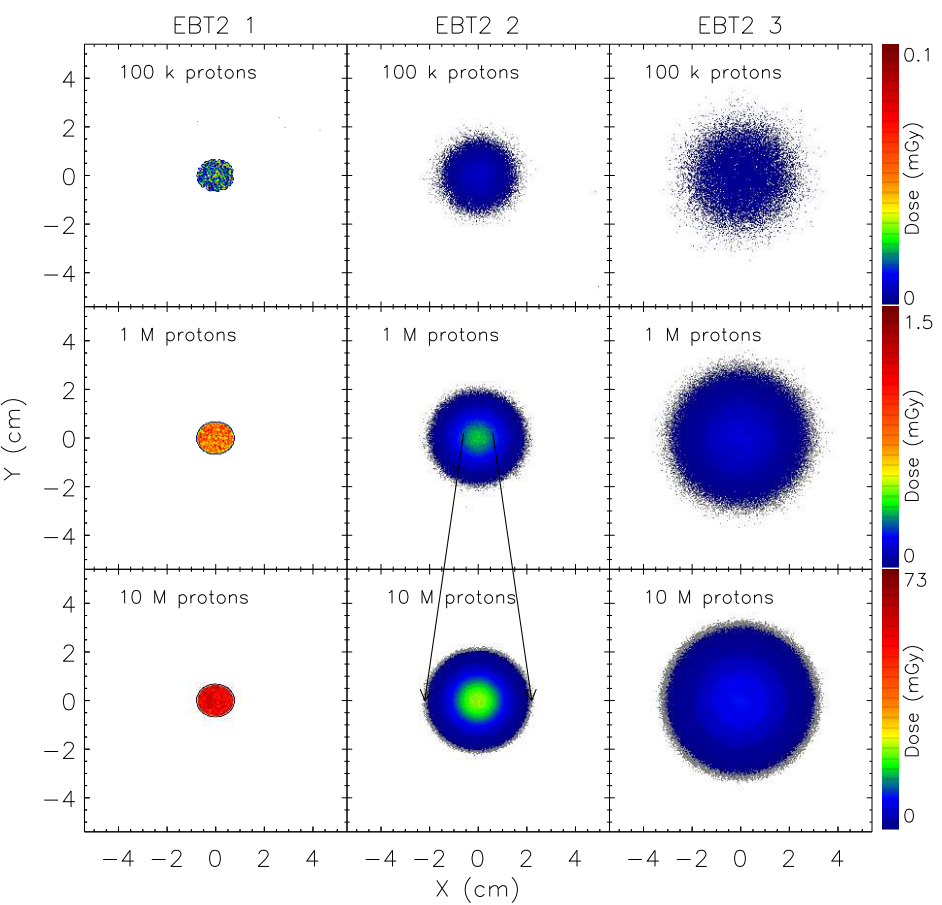

Figure 3.12: Simulation results obtained with the three EBT2 Gafchromatic ${ }^{\circledR}$ films. The beam size and dose are presented for $100 \mathrm{k}, 1 \mathrm{M}$, and $10 \mathrm{M}$ initial protons. 


\section{Chapter 3. Proton beam monitoring system at PET-dedicated cyclotrons}

\subsubsection{Neutron and $\gamma$-ray dose contribution of an irradiation setup}

Figure 3.13 was constructed by using the Geant4 package, and it extends the setup described in section 3.3.3.2. It shows a vacuum-filled aluminum pipe (cyan) that further transports the proton beam (dark blue) produced in the cyclotron (yellow) to a target positioned at its end (white) consisting of an EBT2 film. At the end of the 2-m-long aluminum pipe a circular hole with variable diameter was positioned. Two $50-\mu \mathrm{m}$-thick Havar ${ }^{\circledR}$ windows were considered at both ends of the 2-m-long aluminum pipe. The first one separates the vacuum inside the cyclotron $\left(10^{-5}\right.$ mbar $)$ from the vacuum in the long aluminum pipe $\left(10^{-2} \mathrm{mbar}\right)$, while the second one separates the latter from the atmosphere in the bunker. The $18-\mathrm{MeV}$ proton beam (dark blue in figure 3.13) exits the cyclotron through the first Havar ${ }^{\circledR}$ window, travels along the pipe and is shot through the second Havar ${ }^{\circledR}$ window. Thus part of the proton beam passes through the hole and impinges on the target positioned in the air immediately after this collimator.

Green trajectories in figure 3.13 correspond to neutral particles traveling inside the bunker. Most of these neutral particles stop on the concrete bunker walls or on the cyclotron wall. Red trajectories correspond to negative particles ejected from the surrounding material. Finally, the yellow tube represents the iron yoke of the cyclotron.

Figure 3.14 shows the irradiated areas of two Gafchromic ${ }^{\circledR}$ EBT2 films which were positioned by means of Geant 4 directly after the circular hole in the long aluminum pipe (figure 3.13). Such a location is hereafter referred to as the center of the irradiation setup. The films were positioned at the center of the irradiation setup one at a time during two different simulation runs, each with $10 \mathrm{M}$ initial protons generated inside the cyclotron. The diameter of the irradiated area on the left EBT2 film is $1 \mathrm{~mm}$ while that on the right is $3 \mathrm{~cm}$. From the figure it can be noticed that the dose in the film is restricted to the diameter of the circular hole in both cases $(1 \mathrm{~mm}$ and $3 \mathrm{~cm}$, respectively). From these results it can be concluded that most of the energy deposited in the film is due to the protons crossing the film. In other words, the dose contribution from neutrons and $\gamma$-rays created in the surrounding materials is negligible down to at most the $1 \%$ level. This can be concluded by analyzing the dose spots outside the beam regions in the films. The maximum dose on these outer spots reaches $13 \%$ of the maximum dose on target in one pixel having a front area of $200 \times 200[\mu \mathrm{m}]$ only. This dose value in an out-of-target pixel with such small front area is on average unrepresentative of the out-of-target region. Therefore, it is possible to irradiate a selected region of a target down to $1 \mathrm{~mm}$ diameter. 


\subsection{Preliminary characterization of the external proton beam from}

ICNAS cyclotron
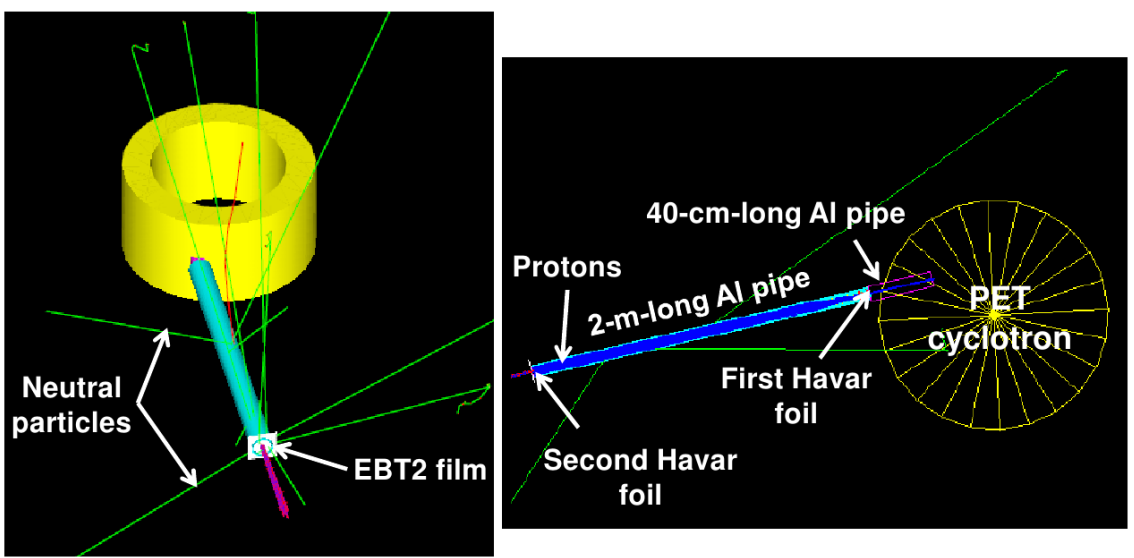

Figure 3.13: Two views (side view (left) and top view (right)) of the irradiation setup simulated with Geant4. The length of the aluminum pipe is $2.4 \mathrm{~m}$ and its diameter is $52.2 \mathrm{~mm}$. The diameter of the circular hole at the end of the aluminum pipe is $3 \mathrm{~cm}$. An EBT2 film (white) of $285-\mu \mathrm{m}$ thickness was positioned in-air directly after this hole.

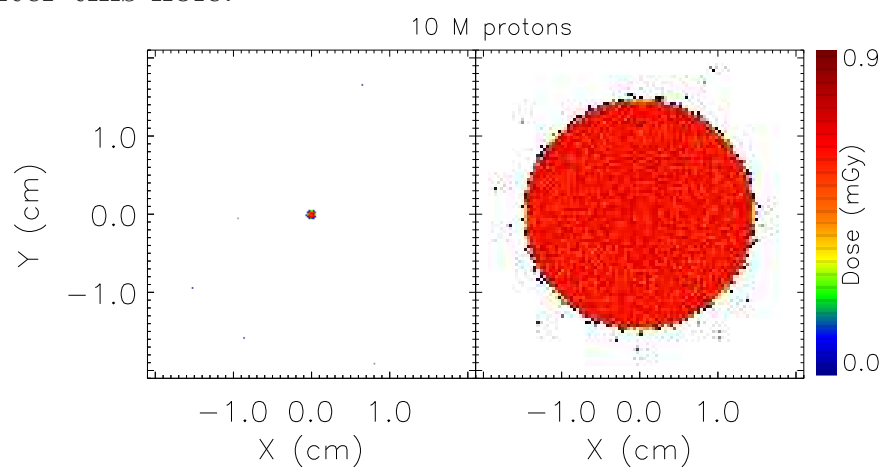

Figure 3.14: Simulation results obtained with EBT2 films positioned directly after the circular hole of the aluminum pipe shown in figure 3.13. The hole diameter was $1 \mathrm{~mm}$ (left) and $3 \mathrm{~cm}$ (right). Ten million protons were simulated which, at a stripper current of $1.5 \mu \mathrm{A}$, corresponds to a film dose rate of $1 \mathrm{kGy} / \mathrm{s}$ at the plateau.

Because the protons cross the film without stopping in it, the energy deposited in the film is not at the Bragg peak region but at the plateau. As shown for example in figure 3.4 the physical dose in water of an $18-\mathrm{MeV}$ proton beam at the Bragg peak is a factor 3 larger than at the plateau. Therefore, the dose values shown in figure 3.14 should be multiplied by 3 if the beam would stop in the film. 


\section{Chapter 3. Proton beam monitoring system at PET-dedicated cyclotrons}

\subsubsection{Assessing beam uniformity}

In order to investigate the possibility of utilizing the $18-\mathrm{MeV}$ proton beam from the PET cyclotron to irradiate a selected region of a target with 1- mm diameter, the Geant4 simulation toolkit was used. For that, a stacked target consisting of 9 EBT2 films was considered after an aluminum collimator with 1- mm diameter at the end of the long aluminum pipe of the irradiation setup (figure 3.13). Furthermore, the proton beam uniformity was assessed.

In addition to simulation, an experimental setup (figure 3.15) has been assembled at ICNAS to investigate the possibility of utilizing the $18-\mathrm{MeV}$ proton beam to irradiate a selected region of a target with 1-mm diameter. For that, two 2 -cm-thick aluminum squares, each with $9 \times 9 \mathrm{~cm}^{2}$ front area and 1 - $\mathrm{mm}$ diameter circular hole located at the center of the square, have been used. Three $4 \times 4 \mathrm{~cm}^{2}$ Gafchromic ${ }^{\circledR}$ EBT2 film sheets were positioned perpendicularly to the beam axis at different distances in front of one of the exit ports of ICNAS cyclotron. The first film was placed at the outer surface of the exit port, the second one was positioned between the two aluminum squares, whereas the third one was placed at the outer surface of the second aluminum square. A photograph of the experimental setup before and after irradiation is shown in figure 3.15. Dose and beam uniformity on the films were studied, together with the undesirable dose effects of neutrons and $\gamma$-rays (neutral particles represented by green trajectories in figure 3.13 ).

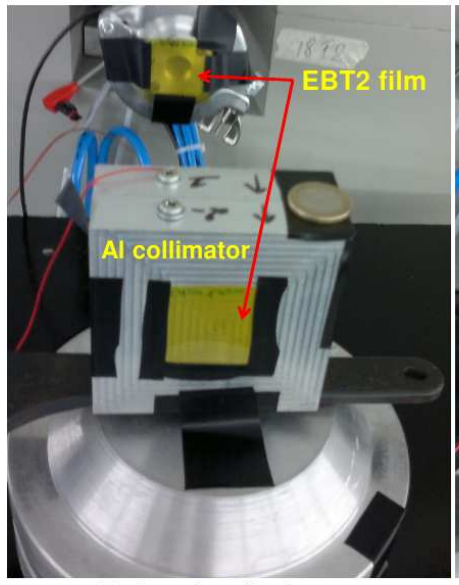

Before irradiation

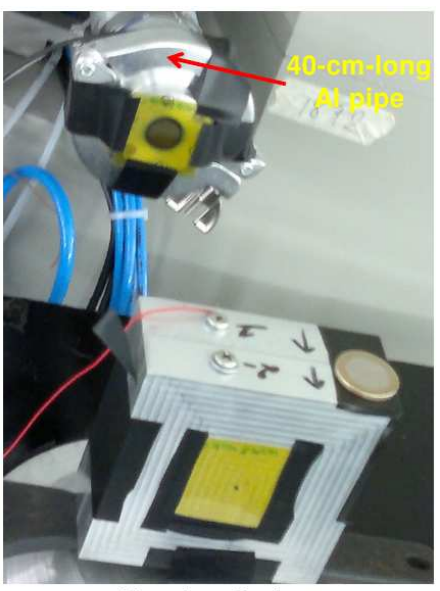

After irradiation

Figure 3.15: Two photographs before (left) and after (right) irradiation of the experimental setup of the aluminum collimators experiment. Two aluminum squares and three EBT2 films have been used.

Figure 3.16 shows the Geant4 simulation results obtained for nine EBT2 films 


\subsection{Preliminary characterization of the external proton beam from}

ICNAS cyclotron

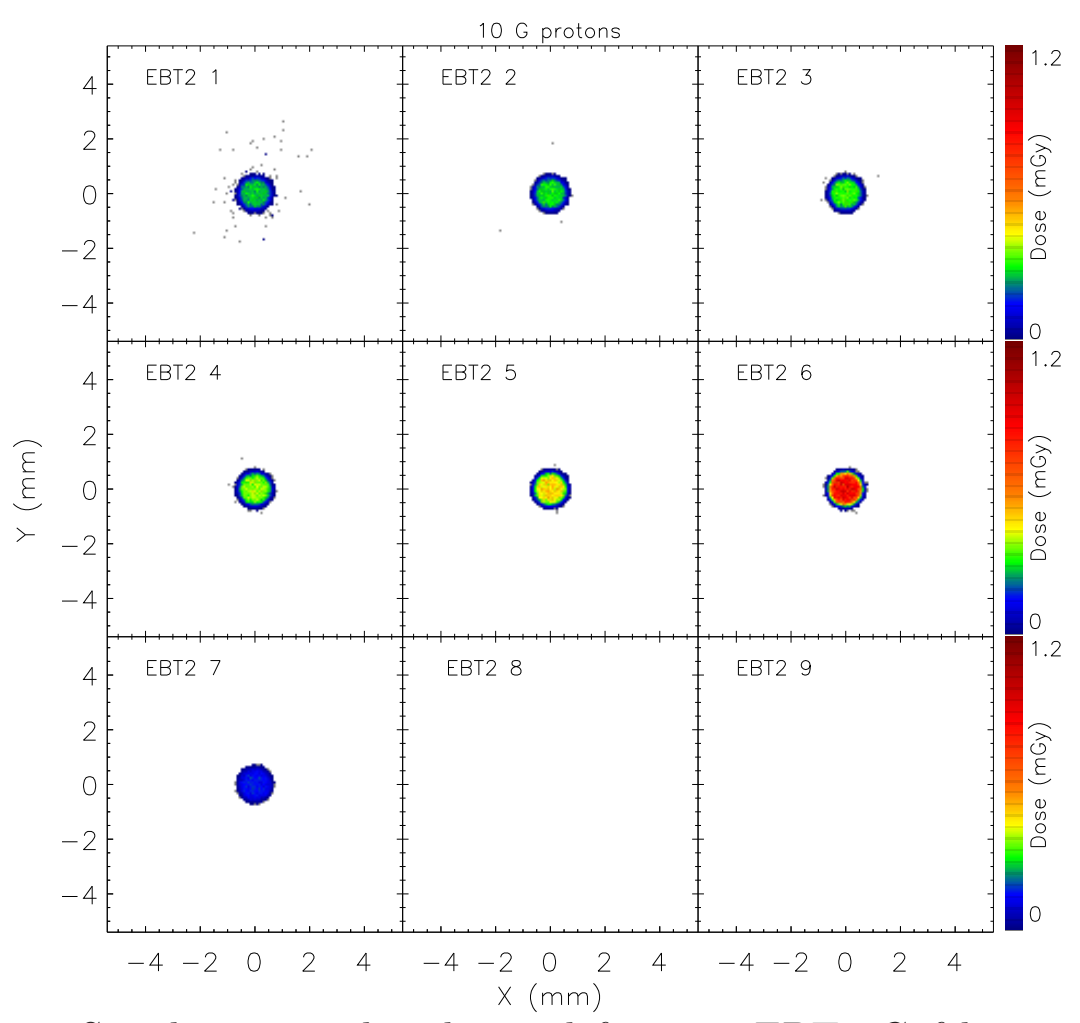

Figure 3.16: Simulation results obtained for nine EBT2 Gafchromatic ${ }^{\circledR}$ films (figure 3.13 ). The beam size and dose are presented for $10^{10}$ initial protons.
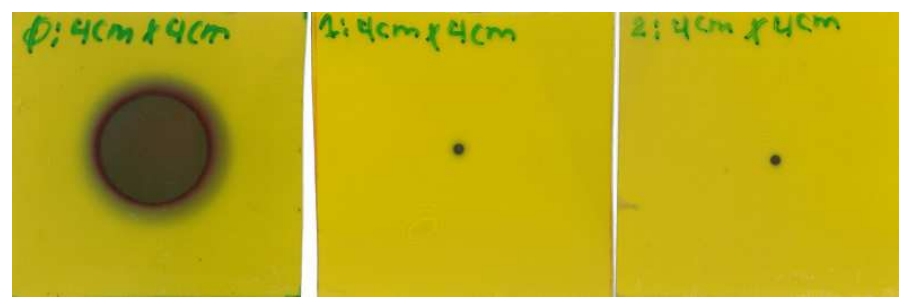

Figure 3.17: Experimental results obtained with three EBT2 films. The films were positioned before, between, and after the two aluminum collimators from left to right, respectively. The color of the irradiated area in each film changed to black when exposed to the proton beam.

positioned in air, after the 1-mm-diameter circular hole. The irradiated area on the films is represented and it can be noticed that the dose in each film is restricted to a $1 \mathrm{~mm}$ diameter region. By analyzing the dose spots in the films, it was found that the dose in the Bragg peak region (sixth film) is a factor three larger than the dose at the plateau (films 1 to 5). Furthermore, the beam size in the sixth film is slightly larger than the beam size in the previous ones. This is expected due to lateral beam straggling, maximum at the Bragg peak. Moreover, figure 3.16 
shows that the dose is homogeneous in the beam region, with a sub-millimetric radial fall off down to negligible dose outside the irradiated spot.

On the other hand, figure 3.17 shows the experimental results obtained with three EBT2 films (figure 3.15). It can be seen that the dose on the third film, which was positioned after the 1-mm-diameter aluminum collimator, is extremely homogenous and restricted to the $1 \mathrm{~mm}$ diameter region (i.e., the beam spot is very sharp). These results are consistent with the simulation ones. They are very important for radiobiological and dosimentric studies, e.g., when irradiating a small tumor xenotransplanted into a mouse.

\subsection{Summary and outlook}

A cyclotron facility has been recently installed at ICNAS for production of radioisotopes for medical use. The accelerator is an IBA model Cyclone ${ }^{\circledR}$ 18/9-HC with fixed energy of $18 \mathrm{MeV}$ for protons and $9 \mathrm{MeV}$ for deuterons. Such facility is equipped with eight exit ports suitable for scientific research. The rationale for performing radiobiological and dosimetric studies among others requires highprecision dosimetry and controlled knowledge about on-target and out-of-target dose. From the experimental results shown, together with Geant4 extrapolations showing possible further improvements regarding out-of-yoke beam delivery, it was concluded that it is possible and feasible to use the cyclotron proton beam to perform such studies.

Measured beam profiles show good qualitative agreement with simulations performed with Geant4. In this chapter we proved by simulation and experiment that we are able to use the $18 \mathrm{-MeV}$ proton beam from the PET cyclotron to irradiate a selected region of a target with $1-\mathrm{mm}$ diameter. The dose contribution from neutron and $\gamma$-ray on a target is negligible down to at least the $1 \%$ level when compared to the proton dose. 


\section{Chapter 4}

\section{Fast and precise verification of proton beam position, range, and energy using a plastic scintillator at PET cyclotrons}

\subsection{Motivation}

Proton beams produced by different cyclotron models can be used in order to perform radiobiological experiments, radiation hardness tests of materials and devices for spacecraft, detector developments, and nuclear physics studies, among others [Papash and Alenitsky, 2008]. Protons and heavier ions are characterized by a property not shared by photons or electrons, the Bragg peak, which allows for a higher deposition of energy in the target volume while lowering the dose to the vicinity. This characteristic may allow improving the experimental results of studies that use proton beams as, for instance, radiobiology and radiophysiology. In addition to the Bragg peak, protons and heavier ions are characterized by a well-defined range with relatively little range scattering in comparison to electrons and X-ray radiation [Kraft, 2000].

Proton beam position, range, and energy measurements are required in order to determine the dose to be deposited into a target volume to be irradiated. Conventional tools for measuring therapeutic proton beam ranges consist of a water phantom and an ionization chamber. However, these tools take a long 

range, and energy using a plastic scintillator at PET cyclotrons

time to be installed, because they are large and heavy Fukushima et al., 2006. Therefore, the need to find fast and precise tools for measuring proton beam ranges is imperative.

The most recent addition to the armory of proton range measurements is the using of an organic radio-fluorogenic gel, which is able to make fixed fluorescent images of the track of the proton beam with spatial resolution better than $0.1 \mathrm{~mm}$ [Warman et al., 2013]. Liquid scintillators are being used for beam range, intensity, and position verification in intensity-modulated proton therapy (IMPT), with submillimeter spatial localization of proton spots delivered in a 3D volume [Archambault et al., 2012]. In addition, plastic scintillators are also being used for the same purposes, where the precision of proton range measurements is about $0.7 \mathrm{~mm}$ at therapeutic ranges [Fukushima et al., 2006].

In summary, studies presented in this chapter were performed using a block of plastic scintillator. The visible scintillation light generated by proton irradiation on the plastic scintillator was recorded with a CCD-based camera, and the length, shapes and brightness distribution were analyzed to obtain longitudinal and lateral distributions.

\subsection{A 40-cm-long aluminum pipe}

\subsubsection{Setup description}

The proton beam is transported outside the cyclotron yoke by means of a 40-cmlong aluminum pipe specially built for that purpose 1 . A $50-\mu$ m-thick Havar ${ }^{\circledR}$ window was fixed at the outer end of the aluminum pipe. In such configuration, this window separates the vacuum inside the pipe $\left(\sim 10^{-5}\right.$ mbar $)$ from the air in the bunker.

\subsubsection{Assessing proton Bragg peak}

The experimental setup which is shown in figure 4.1, left, was assembled at ICNAS for measuring the Bragg peak of the protons delivered by the cyclotron after the 40-cm-long aluminum pipe. In this work, the Bragg peak of the proton beam

\footnotetext{
${ }^{1}$ See section 3.3 .2
} 
from the PET cyclotron was measured by photographing the scintillation light generated when the proton beam irradiated a piece of plastic scintillator, which was positioned in air perpendicularly to the beam path. The overall dimensions (length $\times$ width $\times$ thickness) of the plastic scintillator piece are $2.0 \times 2.0 \times 1.0$ $\mathrm{cm}^{3}$, with its density being $1.02 \mathrm{~g} / \mathrm{cm}^{3}$. This plastic scintillator was covered by black tape in all faces except one in order to reduce the reflection of scintillation light at the edges of the scintillator and at the surrounding material. The generated light was recorded by using a D-link Ethernet-based camera (model no. DCS-910), which was connected to a computer located outside the bunker via an Ethernet cable. The D-link camera was positioned perpendicularly to the scintillator piece as shown in figure 4.1, left. The length, width, and brightness distribution were analvzed offline with the software package ImageJ [ImageJ team, 2004] in order to obtain the range, longitudinal and lateral beam profiles of the proton beam. The aluminum and carbon foils shown in figures 4.1, left, and 4.5 were used to measure the current of the proton beam.
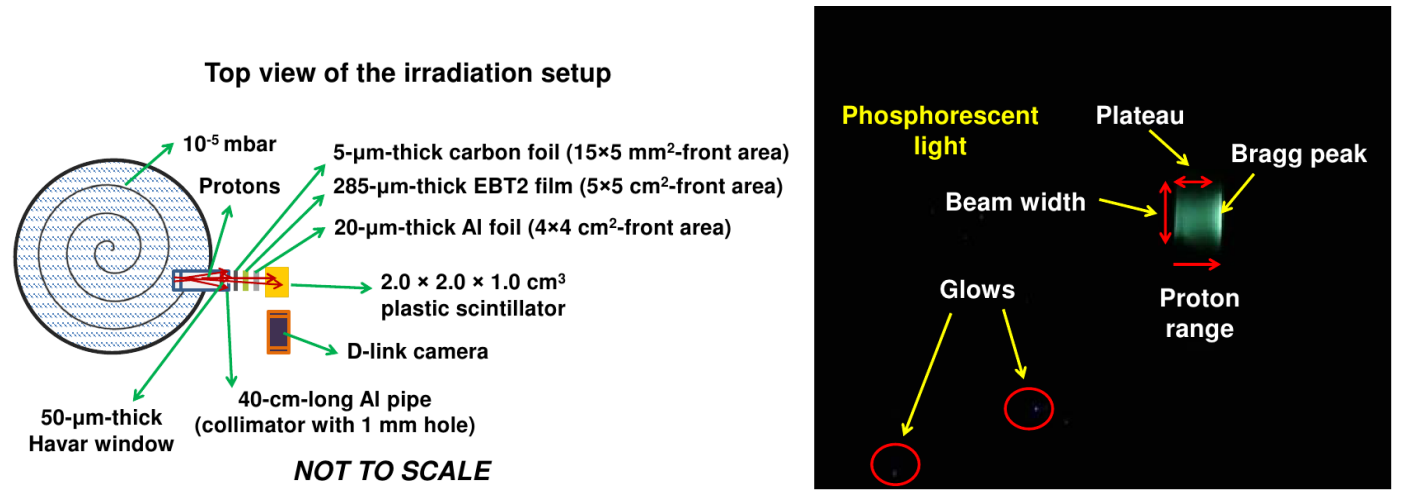

Figure 4.1: Left: Scheme of the experimental setup for the first plastic scintillator experiment. It shows a 40-cm-long aluminum pipe and a plastic scintillator videotaped by a D-link camera. Right: Brightness distribution of phosphorescent light in the plastic scintillator. The plateau and Bragg peak regions can be seen clearly. Particle-induce glowing can be seen at several locations.

The Bragg peak of the proton beam was successfully measured as shown in figure 4.1, right. The plateau and Bragg peak regions can be seen clearly. The image in figure 4.1, right, was taken directly after irradiation, right after stopping the proton beam at the plastic scintillator. Consequently, this corresponds to phosphorescent light generated in the plastic scintillator.

The corresponding longitudinal and lateral beam profiles of the measured image are shown in figure 4.2. The length of proton track within the plastic scintillator to distal $50 \%$ of the Bragg peak was found to be $2.58 \mathrm{~mm}$ (spatial 

range, and energy using a plastic scintillator at PET cyclotrons

resolution $=0.07 \mathrm{~mm}$ ) with $100-20 \%$ post peak fall-off in $0.14 \mathrm{~mm}$. An image for the plastic scintillator (thickness $=1 \mathrm{~cm}$ ) was taken before irradiation, when the light inside the bunker was still on. This image was taken for calibration purposes. It was found that every 14 pixels corresponded to $1 \mathrm{~mm}$ (spatial resolution = $0.07 \mathrm{~mm}$ ). The intensity of the scintillation light at the Bragg peak is a factor of 2.7 larger than that at plateau. From the lateral profiles at the plateau and at the Bragg peak, the FWHM (full width half maximum) of the beam was found to increase from $1.93 \mathrm{~mm}$ to $2.16 \mathrm{~mm}$, with the total intensity of the scintillation light being increased by a factor of 2.5 .
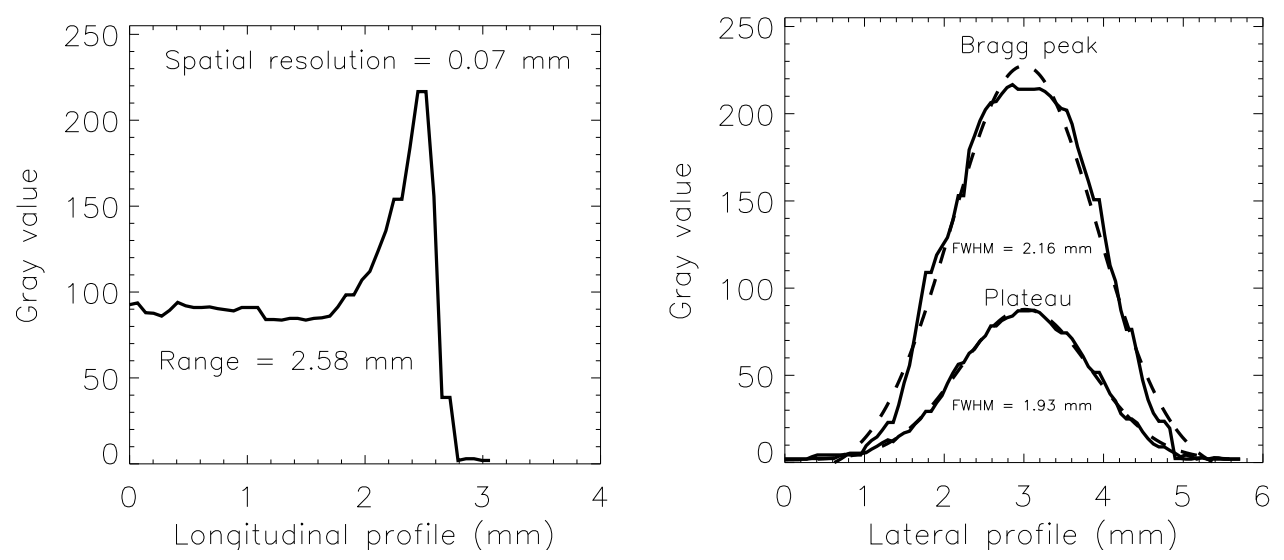

Figure 4.2: Longitudinal (left) and lateral (right) profiles for the measured image of figure 4.1, right. The measured proton range is $2.58 \mathrm{~mm}$. The FWHM of the Gaussian fit (dashed curves) of lateral profiles is 1.93 and $2.16 \mathrm{~mm}$ at the plateau and at the Bragg peak, respectively.

The measured proton beam range in the scintillator, for the material present in the beam path in this experiment (figure 4.1) was found to be $2.58 \mathrm{~mm}$. This range corresponds to a beam energy of $15.45 \mathrm{MeV}$ at the entrance of the plastic scintillator, obtained by using SRIM/TRIM simulations [Ziegler, 2013]. SRIM/TRIM simulation results shown in figure 4.3, report ion range distribution and the proton ionization curve (Bragg peak curve) versus the target depth for a simulation of $15.45 \mathrm{MeV}$ beam incident perpendicularly to a plastic scintillator target. Simulation indicates 2.59 and $0.06 \mathrm{~mm}$ longitudinal range and straggling, respectively. 

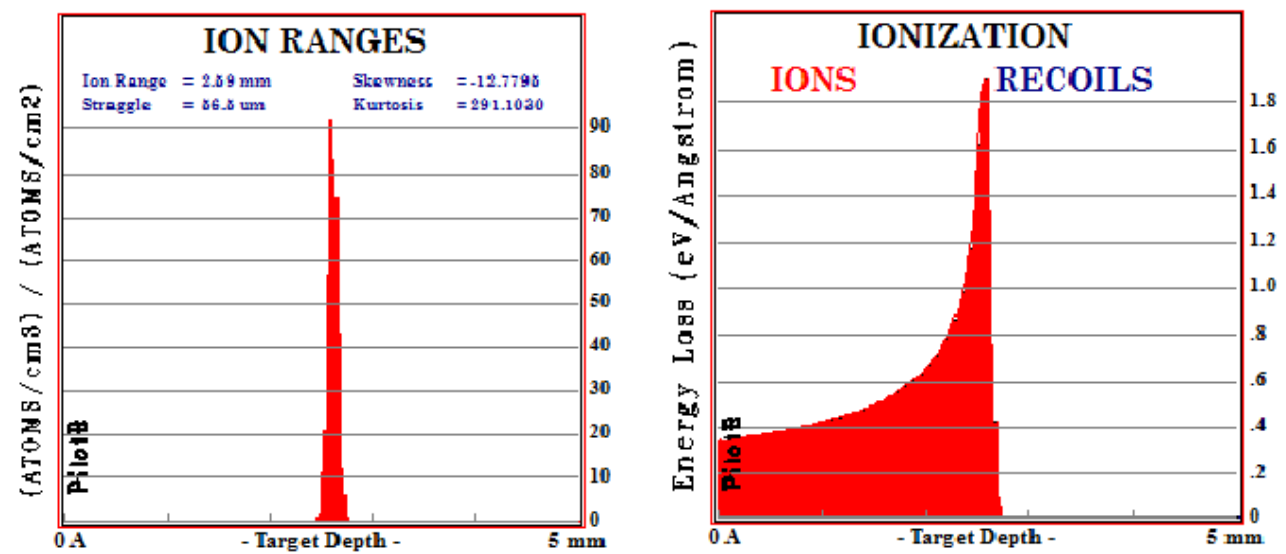

Figure 4.3: Simulation results for $15.45 \mathrm{MeV}$ protons in a block of plastic scintillator. Left image shows ion range distribution, while the right one shows the longitudinal ionization. These results are presented for $10^{3}$ initial protons.

\subsection{A 2.4-m-long aluminum pipe}

\subsubsection{Setup description}

In a first stage the proton beam was brought outside the yoke of the cyclotron using a 40-cm-long aluminum pipe (figure 4.4). A first $50-\mu$ m-thick Havar ${ }^{\circledR}$ window separates the vacuum inside the aluminum pipe $\left(\sim 10^{-5} \mathrm{mbar}\right)$ from the atmosphere in the bunker.

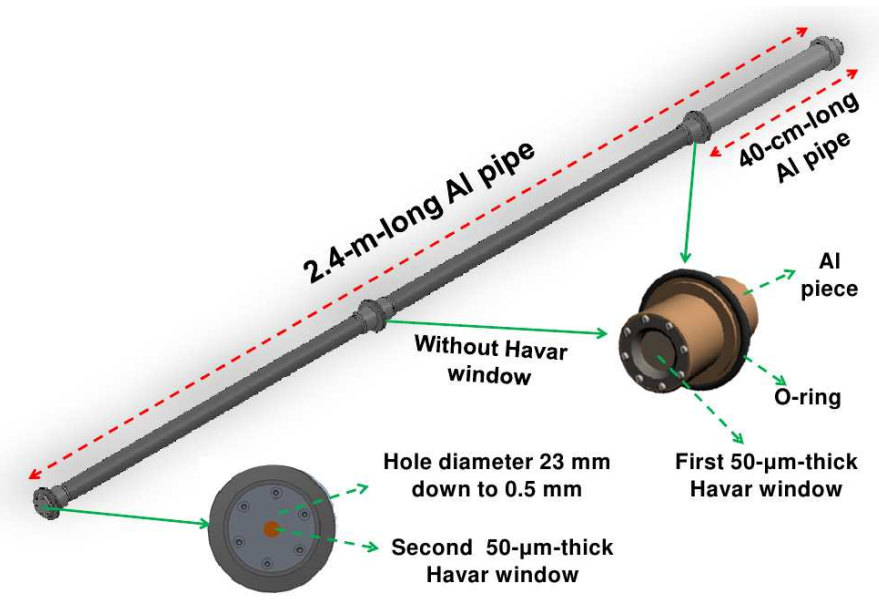

Figure 4.4: Schematic diagram of the 2.4-m-long aluminum pipe. It shows a 40-cm-long aluminum pipe and another two 1-m-long pipes. 

range, and energy using a plastic scintillator at PET cyclotrons

Experimental data 1 and simulations with Geant4 2 reveal that the $50-\mu \mathrm{m}$ thick Havar ${ }^{\circledR}$ window is responsible to a certain degree of beam divergence. In order to take advantage from this fact and to reduce by large the number of protons shoot on a target positioned in air after the 40-cm-long pipe, another 2m-long aluminum pipe was added to the 40-cm-long pipe, as shown in figure 4.4. The 2-m-long aluminum pipe consists of two 1-m-long aluminum pipes fixed to each other without internal windows. The three pipes (the $40-\mathrm{cm}-\mathrm{long}$ pipe and the two 1-m-long pipes) are connected to each other by means of aluminum pieces fitted with an extra O-ring each.

In addition to the first 50 - $\mu$ m-thick Havar ${ }^{\circledR}$ window (30 mm in diameter), which is located at the end of the 40-cm-long aluminum pipe, a second Havar ${ }^{\circledR}$ window was positioned at the end of the 2-m-long aluminum pipe (figure 4.4). This window separates the vacuum inside the 2 -m-long aluminum pipe $\left(\sim 10^{-2}\right.$ mbar) from the atmosphere in the bunker. Right after the second Havar ${ }^{\circledR}$ window, an aluminum collimator with variable diameter $(23 \mathrm{~mm}$ down to $0.5 \mathrm{~mm}$ ) can be screwed to the 2-m-long aluminum pipe. This pipe decreases the total flux of the protons on a target due to beam divergence, since the proton beam spreads itself with distance because of multiple scattering mainly in the first Havar ${ }^{\circledR}$ window.

\subsubsection{Assessing proton Bragg peak}

In the first stage of Bragg peak measurements, the proton beam was transported outside the cyclotron yoke by using the 40-cm-long aluminum pipe (section 4.2). In order to reduce the number of protons arriving on a target, positioned in air after the 40-cm-long pipe, another 2-m-long aluminum pipe was added to the first 40-cm-long pipe (figures 4.4 and 4.5, left). In the second stage of Bragg peak measurements, the experimental setup which is shown in figure 4.5 has been assembled at ICNAS for measuring the location of the Bragg peak of the proton beam after the 2.4-m-long aluminum pipe. The scintillation light (fluorescent light) was recorded for several values of the cyclotron stripper current.

Figure 4.6 shows the measured images for the setup of figure 4.5 at several values of stripper current. It can be seen that the intensity of the scintillation light is increasing with stripper current up to saturation $\left(I_{\text {stripper }}=1.0 \mu \mathrm{A}\right)$. The left image was taken before irradiation, where the light inside the bunker was

\footnotetext{
${ }^{1}$ See section 3.3 .3 .1

${ }^{2}$ See section 3.3 .3 .2 .
} 


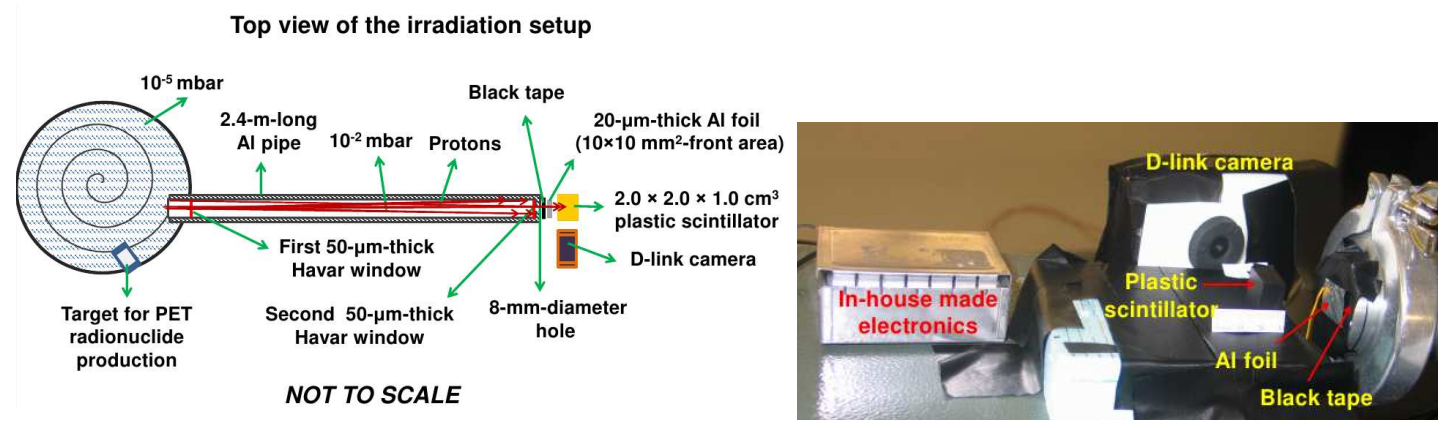

Figure 4.5: Scheme of the experimental setup for the second plastic scintillator experiment. It shows a 2.4-m-long aluminum pipe and a plastic scintillator videotaped by a D-link camera. The photograph on the right shows a detail of the target area where the scintillator was positioned.
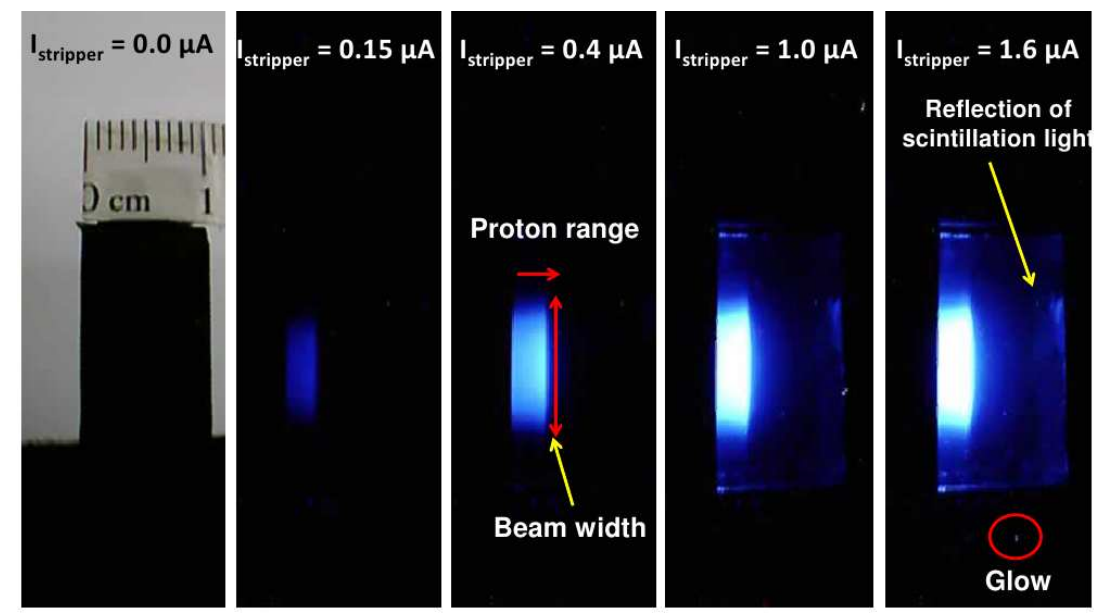

Figure 4.6: Five photographs for the plastic scintillator for the setup of figure 4.5. The left image was taken before irradiation, while the other four were taken during irradiation at different values of stripper current.

still on. This image was taken for calibration purposes, where a printed scale was fixed on the top of the scintillator block. On the other hand, the other four images were taken during irradiation for a stripper current of $0.15,0.4,1.0$, and $1.6 \mu \mathrm{A}$, respectively. The Bragg peak in the middle image $\left(I_{\text {stripper }}=0.4 \mu \mathrm{A}\right)$ can be seen. Moreover, it can be seen that the last two images $\left(I_{\text {stripper }}=1.0\right.$ and 1.6 $\mu \mathrm{A})$ are saturated. The blue light outside the proton range is due to the reflection of the scintillation light inside and at the edges of the plastic scintillator.

Figure 4.7, left, shows the longitudinal beam profiles for the images in figure 4.6 at several values of stripper current. The corresponding lateral beam profiles at the Bragg peak are shown in figure 4.7, right. For the middle image of 
Chapter 4. Fast and precise verification of proton beam position, range, and energy using a plastic scintillator at PET cyclotrons
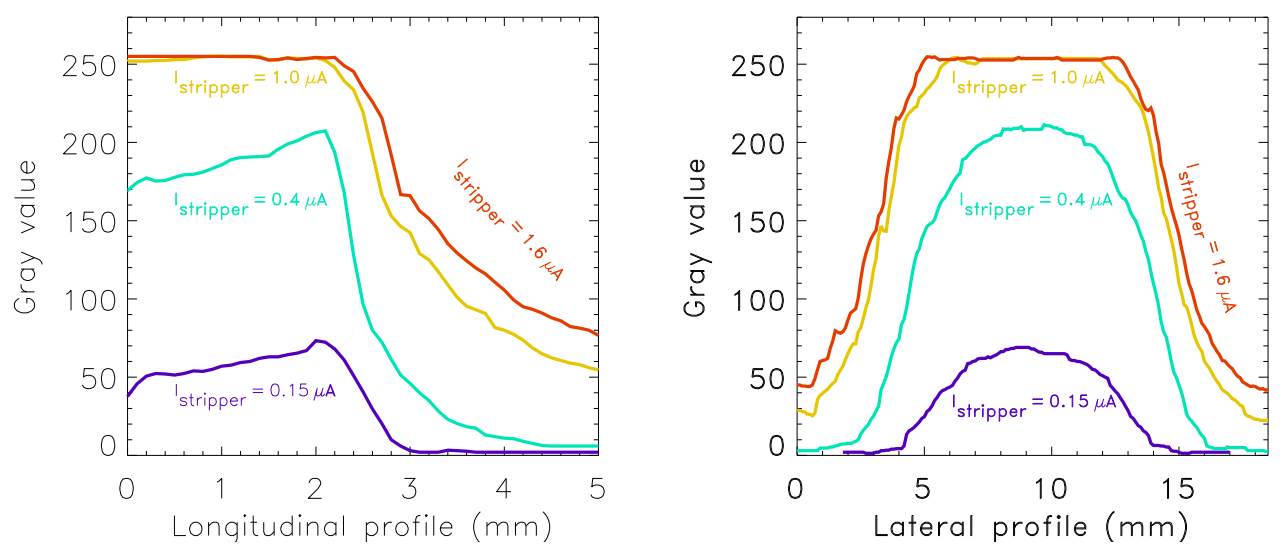

Figure 4.7: Longitudinal (left) and lateral (right) beam profiles for the images of figure 4.6 at $0.15,0.4,1.0$, and $1.6 \mu \mathrm{A}$ stripper current.

figure $4.6\left(I_{\text {stripper }}=0.4 \mu \mathrm{A}\right)$, the measured range of the proton beam within the plastic scintillator to distal $50 \%$ of the Bragg peak is $2.5 \mathrm{~mm}$ (spatial resolution $=0.1 \mathrm{~mm}$, with every 10 pixels corresponding to $1 \mathrm{~mm}$ (figure 4.6, left image)). This range corresponds to a beam energy of $15.2 \mathrm{MeV}$ at the entrance of the plastic scintillator, obtained by using SRIM/TRIM simulations. A decrease of the height of the Bragg peak is observed due to quenching effects (Birks' formula 1 1). Longitudinal beam profiles (figure 4.7, left) do not fall steeply after the Bragg peaks, most probably because of reflection of the generated scintillation light inside and at the edges of the scintillator block during irradiation, as shown in the right image of figure 4.6, for example. Figure 4.8, left, shows lateral beam profiles at the plateau and at the Bragg peak for the middle image of figure 4.6 $\left(I_{\text {stripper }}=0.4 \mu \mathrm{A}\right)$, the FWHM of the beam was found to increase from $9.5 \mathrm{~mm}$ to $9.7 \mathrm{~mm}$, where the intensity of the scintillation light was increased by a factor of 1.2 .

In addition, figure 4.7 shows that the intensity of the scintillation light increases with stripper current, up to saturation, as shown also in figure 4.8, right. By simulating the experimental setups of figures 4.1 and 4.5 with the SRIM/TRIM simulation toolkit, the initial energy of the proton beam was found to be $18 \mathrm{MeV}$. This energy should not be confused with the beam energy at the entrance of the plastic scintillator, which changed between experiments due to the different material present in each case.

\footnotetext{
${ }^{1}$ See section 2.2 .2 .3
} 

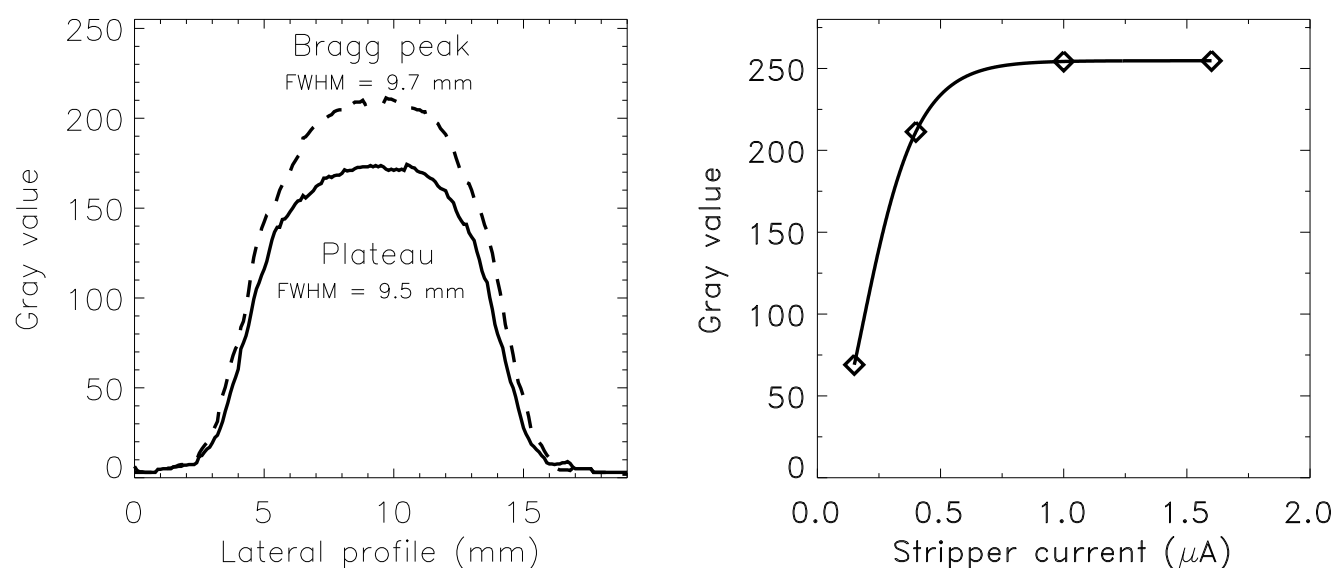

Figure 4.8: Left: Lateral beam profile at the Bragg peak and at the plateau for the middle image of figure 4.6 at $0.4 \mu \mathrm{A}$ stripper current. Right: Gray value as a function of stripper current for the setup of figure 4.5.

\subsubsection{Assessing initial energy of the proton beam}

The experimental setup shown in figure 4.9 has been assembled at ICNAS. The setup was assembled for assessing the initial energy of the proton beam from the PET cyclotron. This was achieved by measuring the range of the proton beam in a plastic scintillator block, and further processing the measured range with the SRIM/TRIM simulation toolkit.

Figure 4.10, left, shows the measured images for the setup of figure 4.9. The left image was taken before irradiation, whereas the other image was taken during irradiation with a stripper current of $0.25 \mu \mathrm{A}$. During irradiation, some particles reached the D-link camera, with some of these particle-induced glowing shown in the right image of figure 4.10, left. Figure 4.10, right, shows the longitudinal and the lateral beam profiles for the right image in figure 4.10, left. The measured range of the proton beam within the plastic scintillator to distal $50 \%$ of the Bragg peak is $2.78 \mathrm{~mm}$ with a spatial resolution 1 of $0.11 \mathrm{~mm}$, with every 9 pixels corresponding to $1 \mathrm{~mm}$ (left image of figure 4.10). This range corresponds to an initial beam energy of $18 \mathrm{MeV}$ obtained by using the SRIM/TRIM simulation toolkit. There is less material in the beam path in respect to the previous setup (figure 4.5), hence the beam range is increased as expected. The lateral profiles at the plateau and at the Bragg peak reveal that the beam width increases with distance in the plastic scintillator, until the end of their range.

\footnotetext{
${ }^{1}$ The way of evaluating the spatial resolution was described in section 4.3.2.
} 

range, and energy using a plastic scintillator at PET cyclotrons

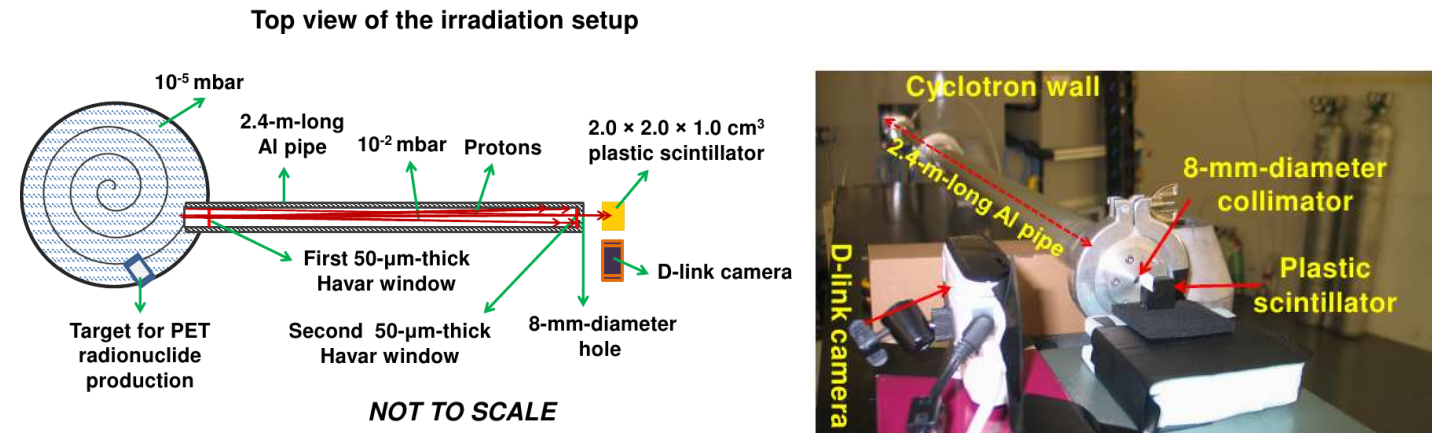

Figure 4.9: Scheme of the experimental setup for the third plastic scintillator experiment. It shows a 2.4-m-long aluminum pipe, an 8-mm-diameter collimator, and a plastic scintillator videotaped by a D-link camera. The photograph on the right shows a detail of the target area where the scintillator was positioned.
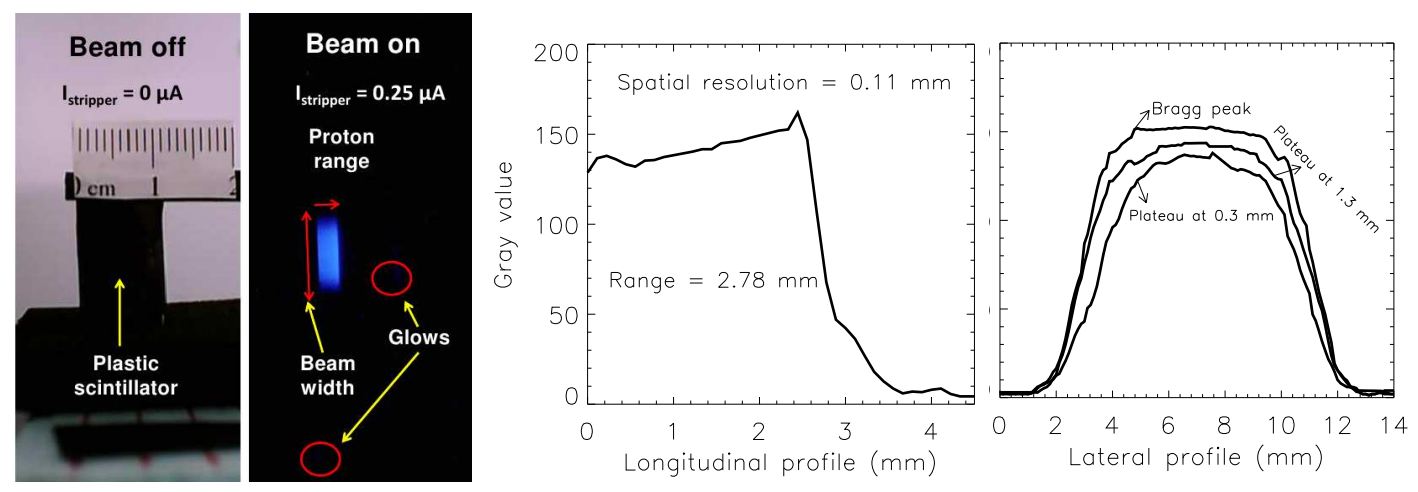

Figure 4.10: Left: Two photographs for the plastic scintillator for the setup of figure 4.9. The left image was taken before irradiation, whereas the right one was taken during irradiation. Right: Longitudinal (left) and lateral (right) beam profiles. The measured proton beam range within the plastic scintillator to distal $50 \%$ of the Bragg peak is $2.78 \mathrm{~mm}$. The lateral beam profiles were assessed at the Bragg peak and at the plateau, i.e., at $0.3,1.3$, and $2.78 \mathrm{~mm}$ from the entrance surface of the scintillator block.

The experimental setup of figure 4.9 was simulated by using the software package SRIM/TRIM (figure 4.11). The simulation results show that the projected range of the proton beam with an initial energy of $18 \mathrm{MeV}$ is $2.89 \mathrm{~mm}$. This range is consistent with the measured one $(2.78 \mathrm{~mm})$.

The setup in figure 4.9 was also used in order to assess the stability of the proton beam energy and intensity. This was achieved by photographing 10 images 

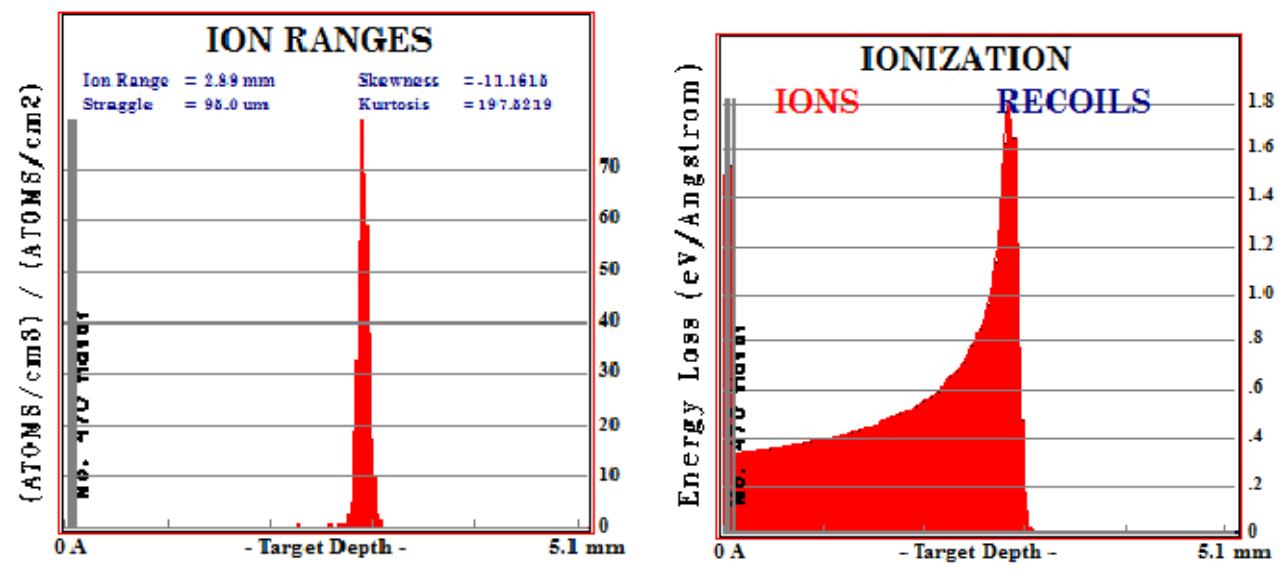

Figure 4.11: Simulation results for the experimental setup of figure 4.9, Left image shows ion range distribution, while the right one shows the longitudinal ionization. These results are presented for $10^{3}$ initial protons with an initial energy of $18 \mathrm{MeV}$. The two 50 - $\mu$ m-thick Havar ${ }^{\circledR}$ foils were considered in addition to the plastic scintillator piece.
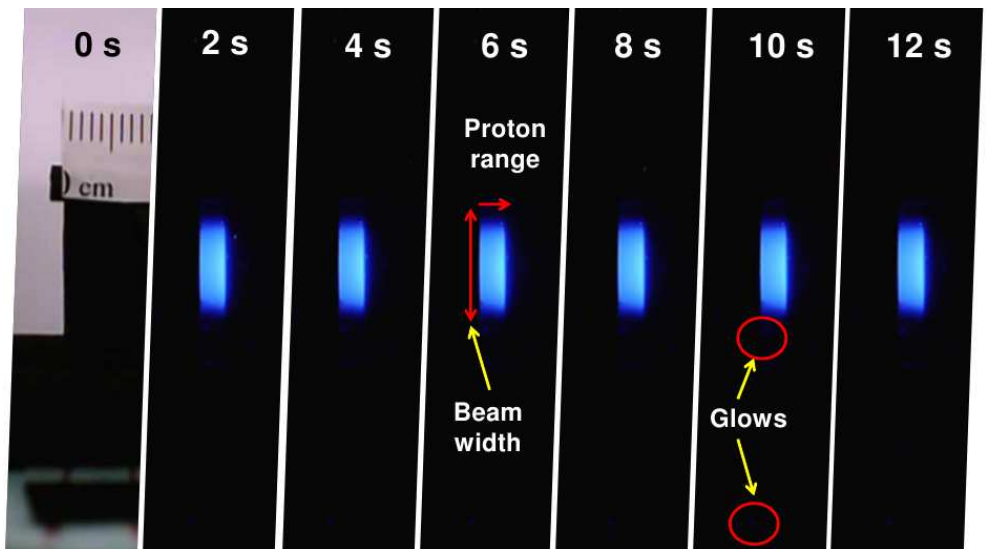

Figure 4.12: Seven photographs for the plastic scintillator for the setup of figure 4.9. The left image was taken before irradiation, while the other six were taken in $12 \mathrm{~s}$ during irradiation, one every $2 \mathrm{~s}$.

of the plastic scintillator during an irradiation-time interval of $20 \mathrm{~s}$, and further processing the resulting images with the software package ImageJ.

Figure 4.12 shows the measured images for the setup of figure 4.9 that have been taken during an irradiation-time interval of $20 \mathrm{~s}$, at a stripper current of $0.25 \mu \mathrm{A}$. The energy stability of the protons from ICNAS cyclotron in an irradiationtime interval of $20 \mathrm{~s}$ are shown in figure 4.13 , left. The range of the proton beam can be measured during irradiation with a standard deviation of $0.05 \mathrm{~mm}$. On 

range, and energy using a plastic scintillator at PET cyclotrons

the other hand, figure 4.13, right, shows the stability of the intensity of the scintillation light in the same time interval. The intensity of the scintillation light can be measured with a standard deviation of 1.06 .
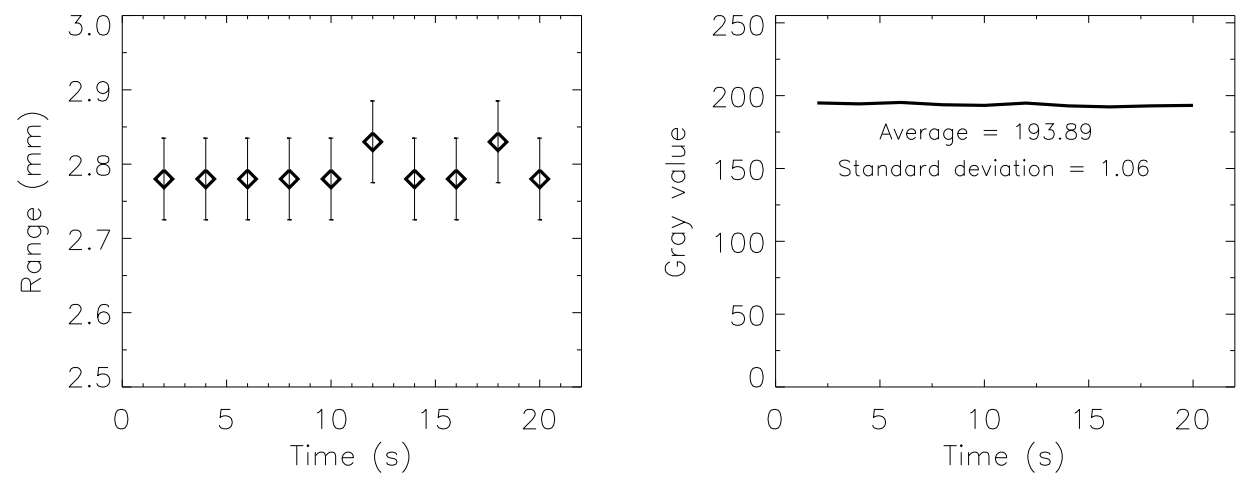

Figure 4.13: Time variation of the proton beam range (left) and of the intensity of the scintillation light (right) for the setup of figure 4.9 at a stripper current of $0.25 \mu \mathrm{A}$. The range varied from $2.78 \mathrm{~mm}$ to $2.83 \mathrm{~mm}$, whereas the intensity varied from 192.3 to 195.3 during an irradiation-time of $20 \mathrm{~s}$.

When the beam current was set to higher values, we verified that there is a radiation damage to the plastic scintillator block as shown in figure 4.14. The proton beam has distorted the scintillator block in two places; the first place with a diameter of $1 \mathrm{~mm}$, whereas the second one with a diameter of $8 \mathrm{~mm}$. The plastic scintillator can be used for a long time if the stripper current is below the $\mu \mathrm{A}$ level. In addition, the beam intensity should be low, i.e., $I_{\text {stripper }}=0.5 \mu \mathrm{A}$ or even less, in order to avoid reflection of scintillation light inside and at the edges of the scintillator block.
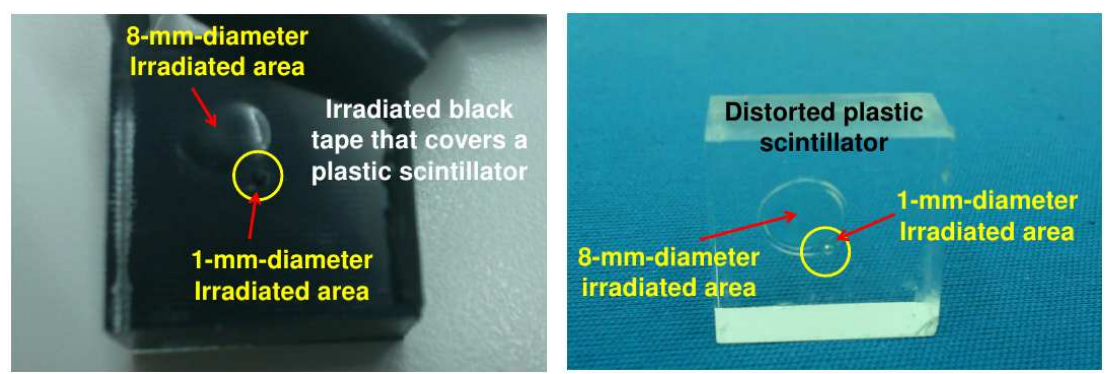

Figure 4.14: Two photographs for the distorted scintillator piece. Left image shows the distorted irradiated area of the black tape that covers the plastic scintillator, whereas the right one shows the distorted areas in the plastic scintillator block. No scintillator distortion was induced if the beam stripper current was left below $0.5 \mu \mathrm{A}$. 


\subsection{Discussion}

The dose rate $D R$ was computed following equation 4.1 Crespo, 2005; Kraft, 2000], by taking into consideration the total beam current delivered on a target, which was measured by means of a thin aluminum foil trespassed by the beam and readout by in-house made transimpedance amplifiers 1 .

$$
D R\left[\frac{G y}{s}\right]=1.6 \times 10^{-9} \cdot \frac{d E}{d x}\left[\frac{k e V}{\mu m}\right] \cdot \phi\left[\frac{1}{s \cdot \mathrm{cm}^{2}}\right] \cdot \frac{1}{\rho}\left[\frac{\mathrm{cm}^{3}}{g}\right]
$$

$d E / d x$ being the stopping power of the incident protons. The stopping power for $15.2-\mathrm{MeV} 2$ protons in the plastic scintillator is $3.355 \mathrm{MeV} / \mathrm{mm}$, which was calculated by using SRIM/TRIM toolkit [Ziegler, 2013]. The density of the plastic scintillator $\rho$ is $1.02 \mathrm{~g} / \mathrm{cm}^{3}$. The measured proton beam current at a stripper current of $0.4 \mu \mathrm{A}$ is $0.62 \mathrm{nA}$, which corresponds to a flux of $3.87 \times 10^{9}$ protons $/ \mathrm{s}$. Consequently, the flux density $\phi$ of the protons at the irradiated area of the plastic scintillator, with 9.5- mm-FWHM at the plateau (figure 4.8, left), is given by equation 6.4 .

$$
\phi\left[\text { protons } /\left(\mathrm{s} . \mathrm{cm}^{2}\right)\right]=\frac{3.87 \times 10^{9}[\text { protons } / \mathrm{s}]}{\pi r^{2}\left[\mathrm{~cm}^{2}\right]}
$$

where $r$ is the radius of the irradiated area $(r=\sigma=F W H M / 2.35)$. As a result, the calculated flux density is about $7.54 \times 10^{9}$ protons $/\left(\mathrm{s} . \mathrm{cm}^{2}\right)$. By substituting these values in equation 4.1, we have obtained the dose rate at the entrance surface of the scintillator block, which is about $25 \mathrm{~Gy} / \mathrm{s}$.

\subsection{Summary and outlook}

In this chapter, we report on experimental results and SRIM/TRIM simulations, which aim at measuring the Bragg peak of the $18 \mathrm{-MeV}$ proton beam from the PET cyclotron. By using a piece of plastic scintillator and a D-link Ethernetbased camera, we measured the proton beam range and width with a spatial resolution of $0.1 \mathrm{~mm}$. The relation between the intensity of the scintillation light, which was generated by irradiation with the proton beam, and the cyclotron stripper current shows good linearity below $0.6 \mu \mathrm{A}$. In addition, we plotted the

\footnotetext{
${ }^{1}$ See chapter 5

${ }^{2}$ This energy corresponds to the assessed beam energy at the entrance of the plastic scintillator for the setup of figure 4.5 .
} 
stability of the proton beam range within the scintillator block. The ranges of the proton beam in the plastic scintillator were used to assess its energy after trespassing one or two Havar ${ }^{\circledR}$, windows and either a 40 -cm-long or a 2.4-m-long aluminum pipe. The initial energy of the proton beam from the PET cyclotron was found to be $18 \mathrm{MeV}$. The dose calculation follows immediately by taking into consideration the total beam current delivered on a target, which was measured by means of a thin aluminum foil trespassed by the beam and readout by in-house made transimpedance amplifiers.

In summary, we provide a fast and precise technique for measuring position, range, and energy of proton beam from a PET cyclotron. This technique can be useful for improving the experimental results of studies that use proton beams as, for instance, radiobiological, radiophysiological and other dosimetric studies. 


\section{Chapter 5}

\section{On-line measurements of beam current from a PET cyclotron using a thin aluminum foil}

\subsection{Motivation}

Proton beam current measurements are required to determine the dose delivered to a target. Dose rate and total dose can be monitored by ionization chambers, which are based on detecting direct ionization created by the passage of protons. Ionization chambers are still the most widely used and most accurate technique, because of their stable operation and wide dynamic range for detection of proton beams [Kim et al., 2006; Renner et al., 1989]. In addition to ionization chambers, the Faraday cup is a widely used method for measuring proton beam current. The principle of operation of a Faraday cup is based on shooting the beam straight into a conducting plate or cup, and conducting the current to a measuring instrument via an electrical lead [Kim et al., 2010].

However, some difficulties appear when measuring beam currents from a PET cyclotron during target irradiation. The beam must not be disturbed by the measuring apparatus and the technique must also be able to measure in short time intervals (a few $\mathrm{Hz}$ to tens of $\mathrm{Hz}$ ) due to eventual cyclotron current oscillations. The nominal energy of the proton beam from ICNAS cyclotron, at the University of Coimbra, is $18 \mathrm{MeV}$. This energy corresponds to a range of $3.45 \mathrm{~mm}$ in water [Ziegler, 2013], therefore the transmission detector used to measure the beam 
current should be thin enough to minimize scattering and energy degradation. A possible solution is the use of a thin aluminum foil (20- $\mu$ m-thick), readout by in-house made transimpedance electronics. The method is based on measuring the secondary electrons liberated from the aluminum foil when protons traverse it (section 5.4).

\subsection{The emission of secondary electrons from a thin foil bombarded with protons}

Conventional systems for ion detection use a thin carbon or metal (e.g., aluminum) foil and a related electronics for detecting electrons ejected from the foil when ions traverse it. The interaction of ions (e.g., protons) with solid targets such as carbon and aluminum foils gives rise to secondary-electron emission from the target [Potiriadis et al., 2005].

The source of the secondary electrons is the energy lost by the protons as a result of ionization processes. The lost energy results in the formation of internal secondary electrons. The majority of these electrons lose their energy in various types of collision processes before reaching the surfaces of the target. Consequently, only a small fraction of all formed electrons are able to reach the surfaces of the foils with sufficient energy to escape from the surfaces (i.e., only electrons that are formed within about $100 \AA$ of the surfaces of the foils can escape from them) [Sternglass, 1957]. Secondary electron emission is the dominant surface yield when $\mathrm{MeV}$-energy protons enter metallic targets Borovsky et al., 1988].

The most important quantity to be measured in this phenomenon is the average number of secondary electrons escaping from the surface of the target per incident ion, i.e., the secondary electron yield $Y$. In case of using a thin foil such as 5 - $\mu \mathrm{m}$-thick carbon or $20-\mu \mathrm{m}$-thick aluminum foil, the protons cross the foil and the electrons are emitted from both surfaces of the solid target. Electron emission from the entrance surface is called backward emission $Y_{B}$, while electron emission from the exit surface is called forward emission $Y_{F}$. The electron emission from both surfaces (entrance and exit) of the target is called total emission (and the total yield is denoted by $Y_{T}$, where the total secondary-electron yield is the sum of the backward yield and forward yield $\left(Y_{T}=Y_{B}+Y_{F}\right)$ [Potiriadis et al., 2005].

Koschar et al. [1989] separated the secondary electrons in two groups: (1) 


\subsection{The emission of secondary electrons from a thin foil bombarded with protons}

low-energy secondary-electrons excited in distant collisions and (2) high-energy $\delta$ electrons excited in close collisions. A fraction $\beta$ of the energy lost by the incident proton is supposed to be lost in close collisions ( $\beta=0.5$ [Sternglass, 1957]), the rest of the lost energy being lost in distant collisions. The low-energy secondaryelectron transport is represented by an exponential decreasing function with a characteristic length $L_{S E}$. which is supposed to be small $\left(L_{S E}=65 \AA\lceil\mathrm{Jung}\right.$ et al., 1996]). The $\delta$-electrons transport energy according to a diffusion function $f\left(x, L_{\delta}\right)=1-\exp \left(-x / L_{\delta}\right)$, where $L_{\delta}$ is a characteristic energy transport length supposed to be comparable to the range of $\delta$-electrons inside the solid target. The value of $L_{\delta}$ is found to increase strongly with increasing projectile velocity and can be described by a simple power law reported in equation 5.1 .Jung et al., 1996].

$$
L_{\delta}=390\left(E_{p} / M_{p}\right)^{1.22}
$$

with $E_{p} / M_{p}$ (projectile energy per mass unit) measured in units of $M e V / u$ and $L_{\delta}$ in units of $\AA$. For the $18 \mathrm{MeV}$ proton beam, $L_{\delta}$ was found to be $1.326 \mu \mathrm{m}$.

For a target of thickness $d$, the forward and backward secondary-electron yields are reported in equations 5.2 and 5.3, respectively [Koschar et al., 1989].

$$
\begin{gathered}
Y_{F}(d)=\Lambda(d E / d x)\left[1-(1-\beta) \exp \left(-d / L_{S E}\right)-\beta \exp \left(-d / L_{\delta}\right)\right] \\
Y_{B}(d)=\Lambda(1-\beta)(d E / d x)\left[1-\exp \left(-d / L_{S E}\right)\right]
\end{gathered}
$$

where $\Lambda$ is a constant depending on the target material (for carbon $\Lambda \approx 10 \mu \mathrm{g} /(\mathrm{keV}$. $\mathrm{cm}^{2}$ ) [Jung et al., 1996]) and $d E / d x$ denotes the electronic stopping power for the incident ions in the target material.

\subsubsection{The emission of secondary electrons from a thin car- bon foil bombarded with protons}

The electronic stopping power $d E / d x$ for the $18-\mathrm{MeV}$ protons in carbon equals $25.51 \mathrm{keV} /\left(\mathrm{mg} / \mathrm{cm}^{2}\right)$. The forward and backward secondary-electron yields for the $18 \mathrm{MeV}$ proton beam incident on a 5 - $\mu$ m-thick carbon foil can be calculated using equations 5.2 and 5.3 , respectively. As a result, the total secondary-electron yield was found to be 0.38 . 


\subsubsection{The emission of secondary electrons from a thin alu- minum foil bombarded with protons}

The proton beam current can be assessed by measuring the current of secondary electrons liberated from a 20- $\mu \mathrm{m}$-thick aluminum foil (section 5.4). However, pure aluminum, which is a soft and lightweight metal, has a dull silvery appearance because of a thin layer of oxidation that forms quickly when it is exposed to air. In 1938, it was found by Bruining and de Boer Borovsky et al., 1988] that the secondary electron yield from oxidized aluminum is a factor 2.25 larger than the secondary electron yield from pure aluminum.

Theoretical prediction of the secondary-electron yield for protons normally incident on pure aluminum surfaces is given by equation 5.4. This expression was termed equation of Sternglass [Borovsky et al., 1988]:

$$
Y_{A l}=1.6 \times 10^{-9}(d E / d x)\left[1+\frac{1}{1+\frac{E}{0.183}}\right]
$$

with $Y_{A l}$ being the yield factor describing the amount of secondary emission per incident proton on the surface of the thin pure aluminum foil (unitless), $d E / d x$ is the stopping power of the incident protons in aluminum $(\mathrm{eV} / \mathrm{cm})$, while $E$ is the energy of the incident protons $(\mathrm{MeV})$. By considering the oxidized aluminum foil equation 5.4 must now be:

$$
Y_{\mathrm{Al}_{2} \mathrm{O}_{3}}=2.25 \cdot Y_{\mathrm{Al}}
$$

The stopping power for $18 \mathrm{-MeV}$ protons in aluminum is $5.802 \mathrm{MeV} / \mathrm{mm}$ i.e., $d E / d x=5.802 \times 10^{7} \mathrm{eV} / \mathrm{cm}$. Therefore, the calculated secondary-electron yield for protons with energy of $18 \mathrm{MeV}$ incident on oxidized aluminum was found to be 0.211 .

\subsection{Assessing proton Bragg peak}

The experimental setup shown in figure 5.1 has been assembled at ICNAS for assessing the range of the protons from the cyclotron based on beam current measurements around the Bragg peak. This was achieved by measuring the current of secondary electrons liberated (secondary current) from several aluminum 
foils positioned in a stacked target. The signals from the aluminum foils of the stacked target were measured by using in-house made electronics (figure 5.2).
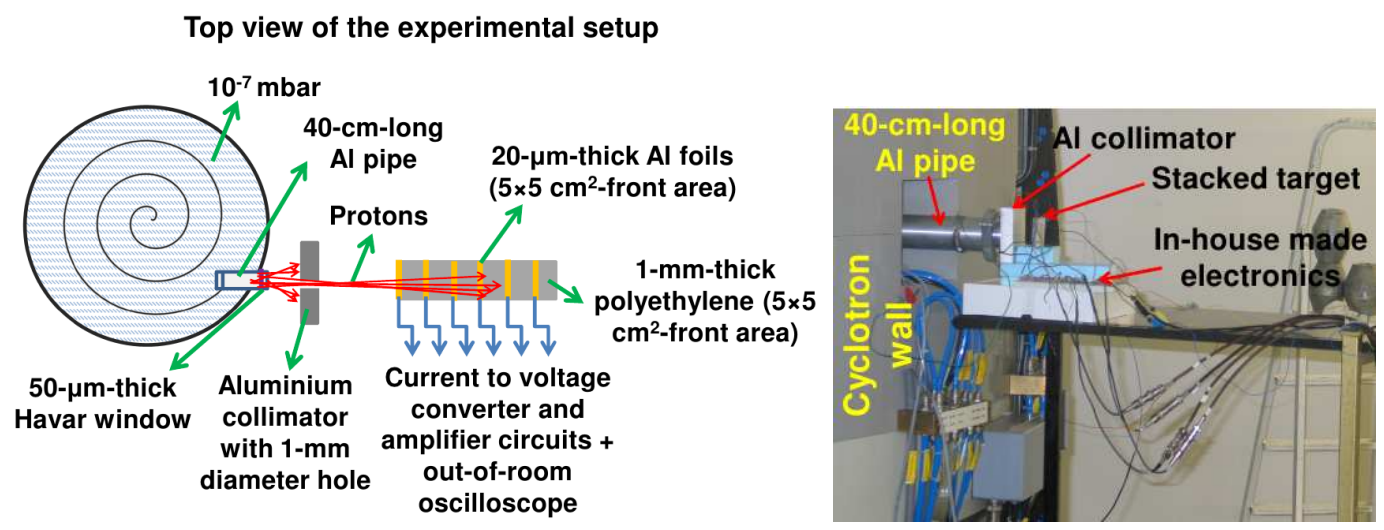

Figure 5.1: Schematic diagram of the experimental setup of the stacked target experiment (left). The scheme shows a 40-cm-long aluminum pipe, an aluminum collimator with 1-mm diameter hole, and a stacked target consisting of 6 aluminum foils interleaved with polyethylene sheets. Photograph of the experimental setup (right). It shows the aluminum collimator, the stacked target, and the in-house made electronics.

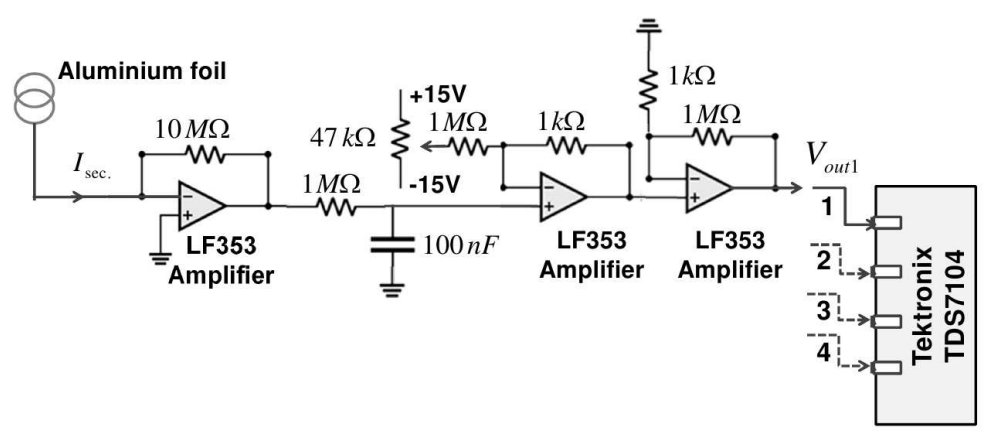

Figure 5.2: Schematic diagram of the in-house made electronics, with total gain of $10^{10}$, that has been used to readout the signals from the aluminum foils of the stacked target. It shows a current to voltage converter followed by a low pass filter, a voltage offset adjustment, a voltage amplifier circuit, and an oscilloscope (Tektronix TDS7104).

An aluminum collimator with 1-mm diameter hole has been positioned directly after the 40-cm-long aluminum pipe, the latter connected to one of the exit ports of ICNAS cyclotron. The stacked target was positioned after the aluminum collimator, at a distance of $2 \mathrm{~cm}$ (figure 5.1).

The stacked target consists of 6 aluminum foils, each with $20-\mu \mathrm{m}$ thickness and with $5 \times 5-\mathrm{cm}^{2}$ front area, interleaved by 6 polyethylene sheets, each with 1 - 


\section{Chapter 5. On-line measurements of beam current from a PET cyclotron using a thin aluminum foil}

mm thickness. In between every two aluminum foils one sheet of polyethylene has been used in order to separate the aluminum foils from each other and to decrease the energy of the incident protons every time they pass through a polyethylene sheet, until they stop in the vicinity of the fourth aluminum foil, as per simulation results for the stacked target experiment by using SRIM/TRIM [Ziegler, 2013].

The signals from the aluminum foils of the stacked target were measured using a current to voltage converter, with amplification factor of $10^{7} \mathrm{~V} / \mathrm{A}$, followed by a low pass filter. The filtered signals were amplified again by using a voltage amplifier with gain of $10^{3}$. Finally, the resulting signals were measured using a Tektronix TDS7104 oscilloscope. The voltage offset adjustment shown in figure 5.2 was used to balance the voltage offset within the range of $\pm 14 \mathrm{~V}$, where the $1 \mathrm{M} \Omega$ resistor injects a current which produces a voltage drop across the 1 $\mathrm{k} \Omega$ resistor, and therefore changes the voltage offset. The output signals were adapted by using the potentiometer $(47 \mathrm{k} \Omega$ ) of the voltage offset adjustment circuit. This in-house made electronics was installed inside an aluminum box for shielding the radiofrequency $(42 \mathrm{MHz})$ of the cyclotron.

Four BNC (Bayonet Neill Concelman) signal cables and one SHV (Safe High Voltage) cable were connected from outside of the bunker by means of an underground plastic tube. Therefore, equipment sensitive to radiation can be installed outside the bunker during measurements.

In the first stage of measurements, the first four aluminum foils of the stacked target have been connected via BNC signal cables to the four channels of the Tektronix TDS7104, with each aluminum foil connected to one channel. Therefore, we were able to measure the signals from the first four foils at the same time. In the second stage, the signals from the last four foils were measured. Therefore, we were able to measure the signals from the last four foils at the same time by the oscilloscope. For both measurement stages, the cyclotron stripper current 1 has been kept at a constant value of $2.5 \mu \mathrm{A}$.

The signals from the first three and the last three aluminum foils of the stacked target experiment (figure 5.1) are shown in figures 5.3 and 5.4, respectively. These two figures show that the signals from the six aluminum foils are shifted downwards during irradiation (beam on). For instance, it can be seen clearly that the signal of foil no. 4 (figure 5.4) was shifted downwards by about $11.2 \mathrm{~V}$, while the signal of foil no. 6 was shifted downwards by just about $0.3 \mathrm{~V}$. This is expected from the simulation results that have been obtained by using the SRIM/TRIM simulation toolkit for the stacked target experiment (figure 5.5).

\footnotetext{
${ }^{1}$ See section 3.1.1
} 


\subsection{Assessing proton Bragg peak}
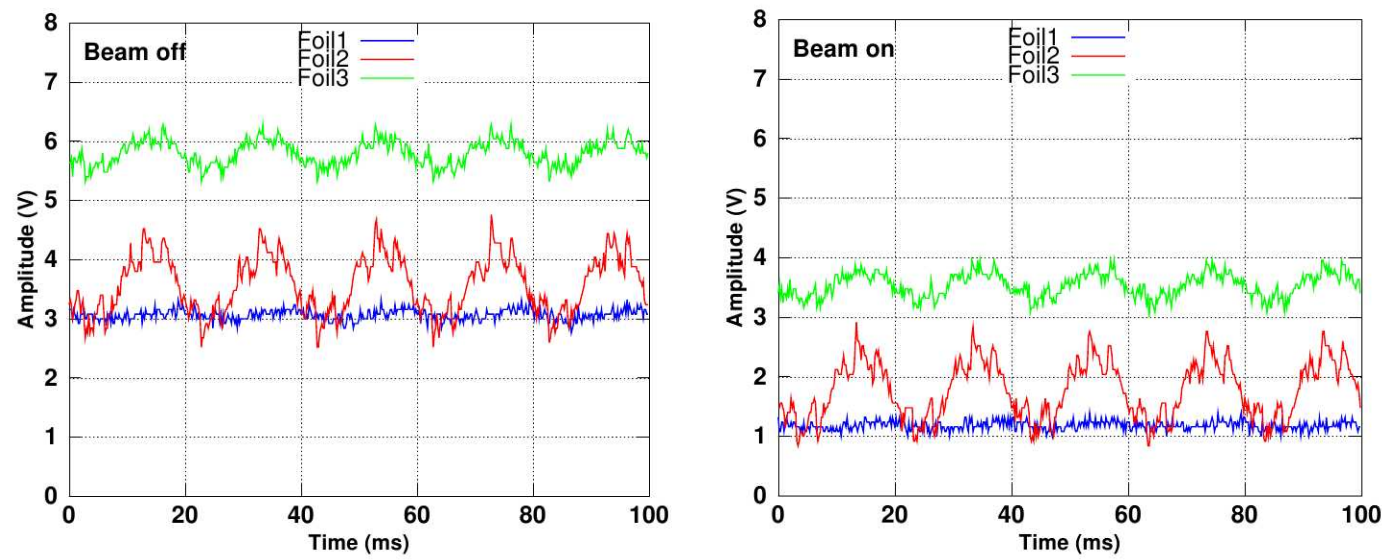

Figure 5.3: Signals from the first three aluminum foils of the stacked target setup of figure 5.1. The left signals were acquired when the proton beam was off (before irradiation). The RF (radiofrequency), the magnetic field, and the ion source of ICNAS cyclotron were on. The right ones were acquired when the proton beam was on (during irradiation). The signals from the three foils were shifted downwards during irradiation by $1.88,1.79$, and $2.26 \mathrm{~V}$, respectively.
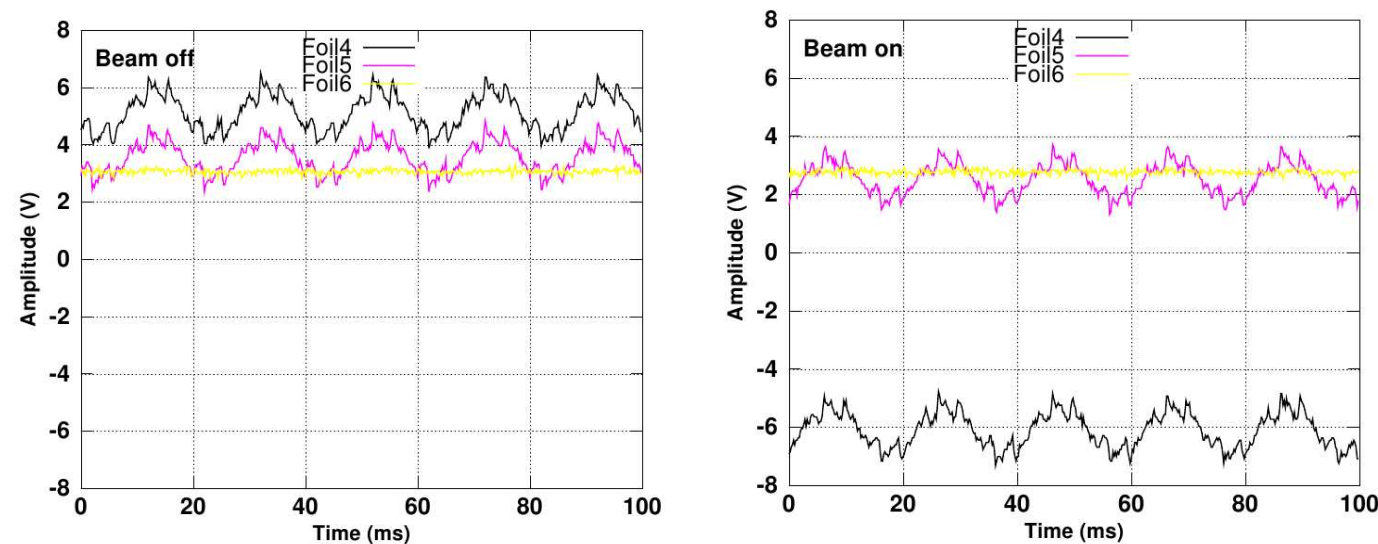

Figure 5.4: Signals from the last three aluminum foils of the stacked target setup of figure 5.1. The left signals were acquired when the proton beam was off (before irradiation). The RF (radiofrequency), the magnetic field, and the ion source of ICNAS cyclotron were on. The right ones were acquired when the proton beam was on (during irradiation). The signals from the three foils were shifted downwards during irradiation by $11.22,1.04$, and $0.29 \mathrm{~V}$, respectively.

In figure 5.5, the six aluminum foils are represented by the black vertical lines (left image) and by the gray vertical lines (right image). In between every two aluminum foils a sheet of polyethylene was considered with $1 \mathrm{~mm}$ thickness. Figure 5.5 shows that the majority of the protons stop in the vicinity of the fourth 
aluminum foil. As a result, the protons lose most of their energy in the vicinity of the fourth foil. Therefore, the signal from the fourth foil (figure 5.4) has the largest voltage shift compared to the others. In addition, the projected range of the proton beam, with energy of $18 \mathrm{MeV}$ is $3.08 \mathrm{~mm}$ in the stacked target.
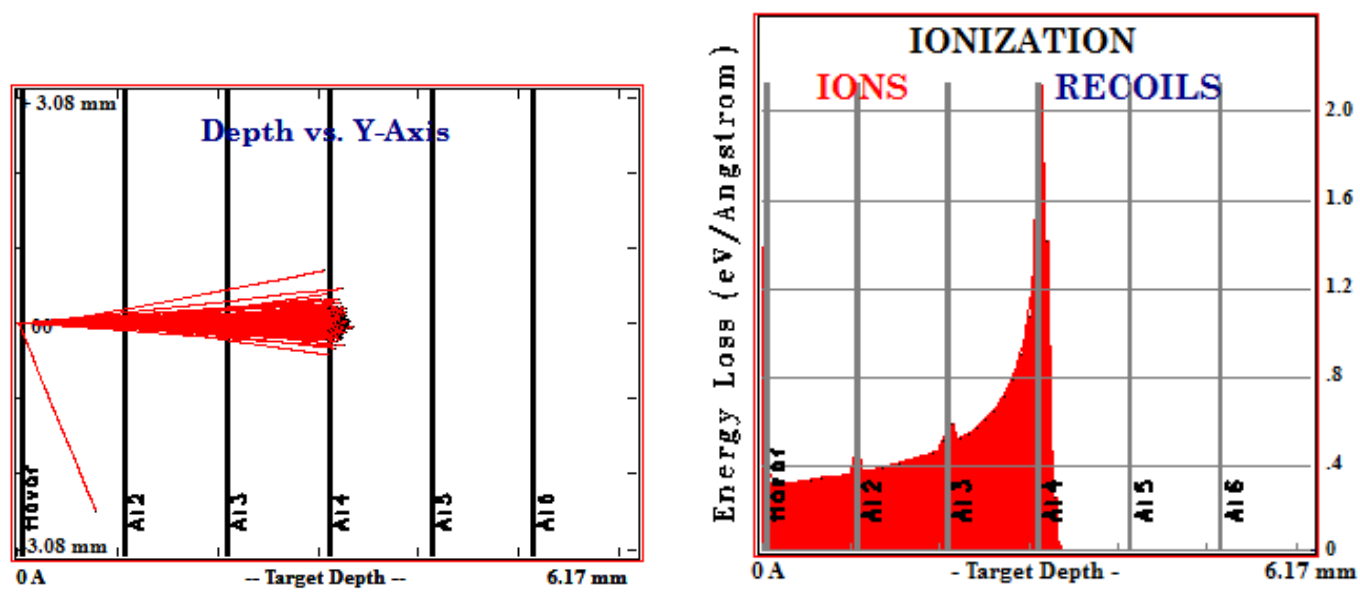

Figure 5.5: Simulation results for the experimental setup of the stacked target experiment (figure 5.1). Left image shows the XY longitudinal particle distribution, while the right one shows the longitudinal ionization. The results were obtained by using the SRIM/TRIM toolkit. These results are presented for $10^{3}$ initial protons with an initial energy of $18 \mathrm{MeV}$. The $50-\mu$ m-thick Havar ${ }^{\circledR}$ foil was considered in addition to the aluminum and polyethylene stacked target.

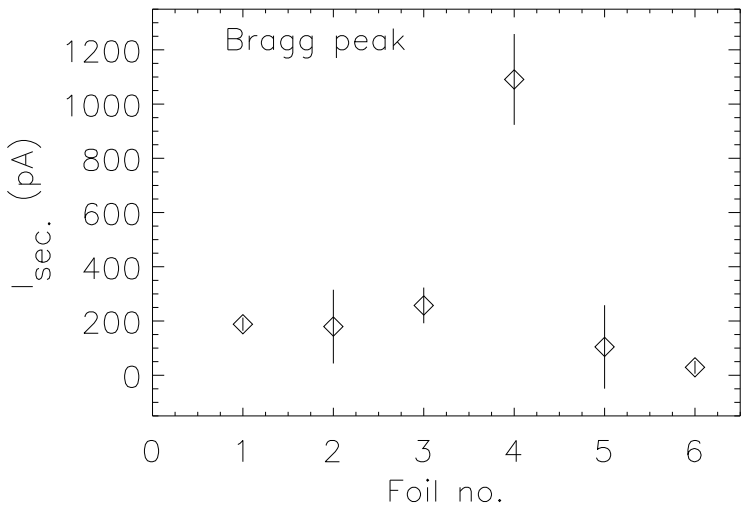

Figure 5.6: Secondary current with error bars as a function of foil number for protons from ICNAS cyclotron measured with the setup of figure 5.1 .

The setup shown in figure 5.1 allows for measuring the secondary current at different values of beam energy, particularly in the vicinity of the Bragg peak. The measured Bragg peak of the proton beam from ICNAS cyclotron is shown in 
figure 5.6. Simulation results (figure 5.5) for the stacked target experiment show that part of the Bragg peak of the protons from the cyclotron has been measured by the fourth aluminum foil. The measured proton Bragg peak (figure 5.6) is consistent with the one simulated with SRIM/TRIM (figure 5.5, right). The Bragg peak to plateau ratio is about six for both the simulated and the measured peaks. Furthermore, the projected range of the protons with $18 \mathrm{MeV}$ by using SRIM/TRIM simulation is $3.08 \mathrm{~mm}$ in the stacked target, which is in agreement with the measured one $(\sim 3.08 \mathrm{~mm})$.

\subsection{Assessing beam current using a thin alu- minum foil}

Figure 5.7 shows the experimental setup that has been assembled at ICNAS for assessing the beam current of the protons from ICNAS cyclotron. The beam current on target was assessed by measuring the current of secondary electrons liberated (secondary current) from a 20- $\mu$ m-thick aluminum foil.

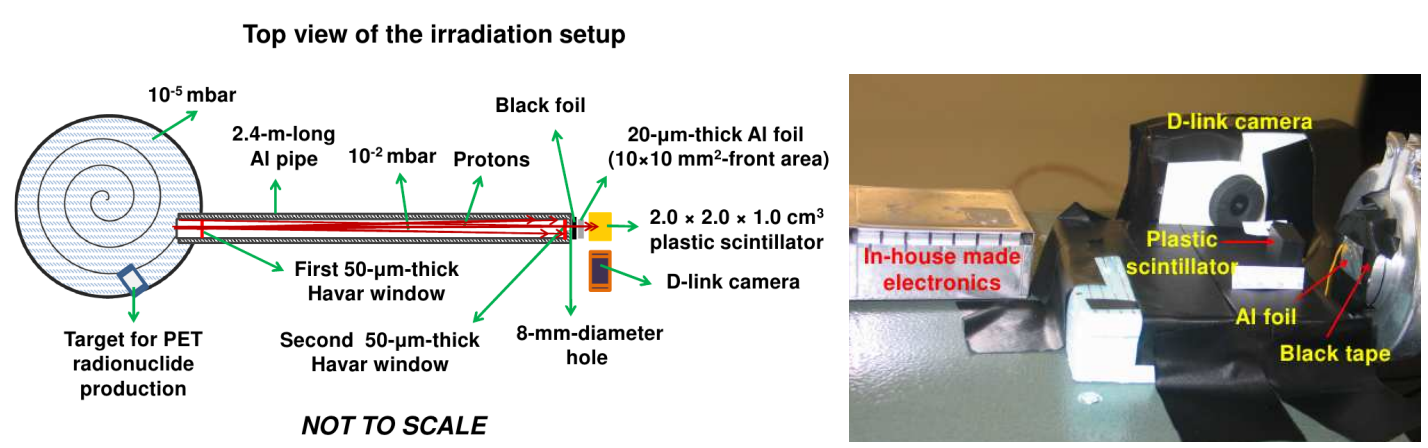

Figure 5.7: Schematic diagram of the experimental setup of the aluminum foil experiment (left). It shows a 2.4-m-long aluminum pipe, a piece of black foil, an aluminum foil, and a plastic scintillator. Photograph of the experimental setup (right).

The 2.4-m-long aluminum pipe (figures 4.4 and 5.7) permits the proton beam produced in the cyclotron to reach a target at its end, where the beam enters through an 8-mm-diameter hole (figure 5.7).

The 8-mm-diameter hole was covered by a piece of black foil in order to prevent the secondary electrons liberated from the aluminum collimator and the second Havar ${ }^{\circledR}$ window from reaching the aluminum foil. The $20-\mu$ m-thick aluminum foil was fixed at the outer surface of the black foil in alignment with 


\section{Chapter 5. On-line measurements of beam current from a PET cyclotron using a thin aluminum foil}

the 8-mm-diameter hole. Then, the signal from the aluminum foil was amplified and connected via a 25-m-long BNC cable to an oscilloscope (PicoScope 2203) positioned outside the bunker. On the other hand, a plastic scintillator was positioned in air perpendicularly to the beam axis after the aluminum foil at a distance of about $2 \mathrm{~cm}$. The visible scintillation light was recorded by using a D-link Ethernet-based camera (model no. DCS-910), which was positioned perpendicularly to the scintillator piece as shown in figure 5.7 .

The D-link camera was connected to a computer located outside the bunker. Therefore, we were able to monitor the scintillation light during irradiation. The plastic scintillator was used in order to make sure that the proton beam is passing through the aluminum foil during irradiation by seeing the generated scintillation light from the plastic scintillator.

The aluminum foil was connected to a modified in-house made electronics (figure 5.8), where an $11^{\text {th }}$ order Chebyshev low pass filter was used in order to attenuate undesired $50 \mathrm{~Hz}$ frequency noise that would otherwise decrease the voltage dynamic range and increase the error percentage in the measurements. The $11^{\text {th }}$ order Chebyshev low pass filter is shown in figure 5.8 between $V_{\text {out } 1}$ and $V_{\text {out }}$. The AC analysis for the in-house made electronics of figure 5.8 is shown in figure 5.9, which was obtained by using the LTspice toolkit [LTspice, 2013]. In

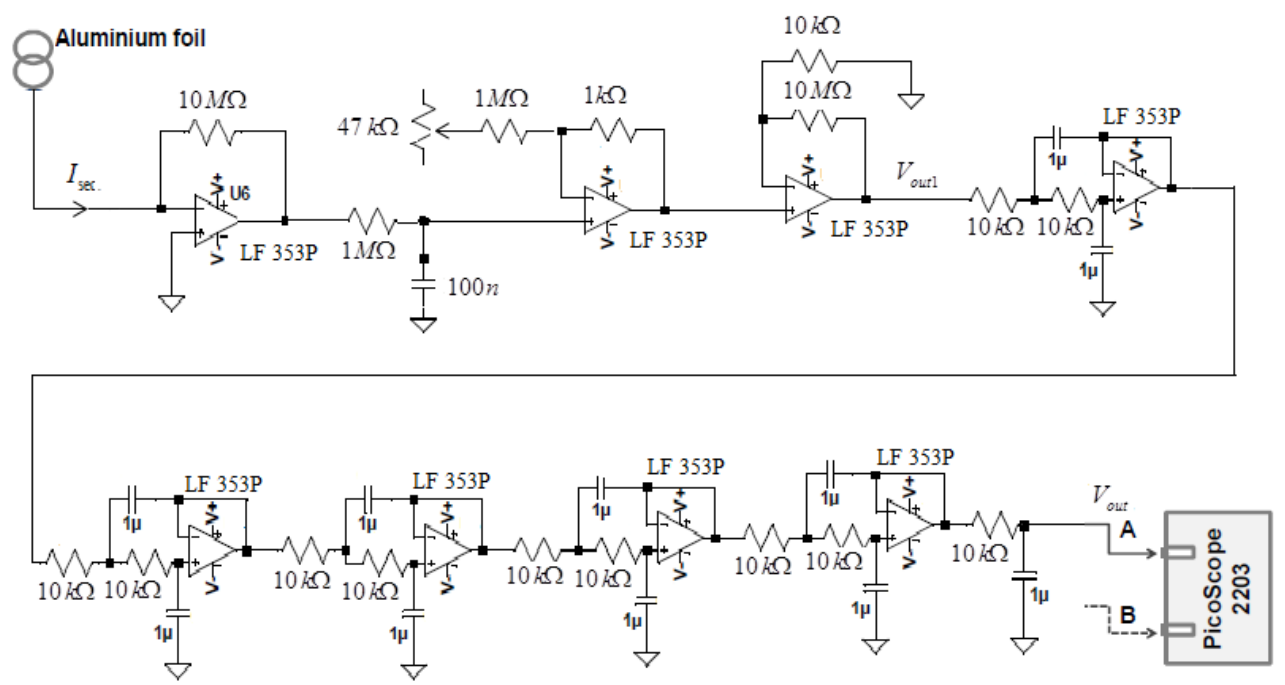

Figure 5.8: Schematic diagram of the in-house made electronics, with total gain of $10^{10}$, that has been used to readout the signals from the thin aluminum foil (figure 5.7). It shows a current to voltage converter followed by a low pass filter, a voltage offset adjustment, a voltage amplifier circuit, an $11^{\text {th }}$ order Chebyshev low pass filter, and an oscilloscope (PicoScope 2203) positioned outside the bunker. 
figure 5.9 it can be seen that the gain at $100 \mathrm{mHz}$ is $198.5 \mathrm{~dB}$, whereas at $50 \mathrm{~Hz}$ it is $67.1 \mathrm{~dB}(\sim 3.7 \mathrm{M}$ attenuation factor $)$.

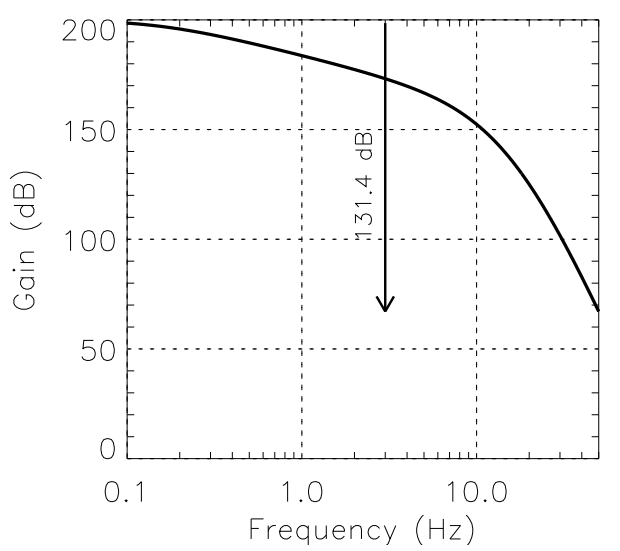

Figure 5.9: AC analysis of the output voltage with the in-house made transimpedance electronics followed by the $11^{\text {th }}$ order Chebyshev low pass filter (figure 5.8). This analysis was obtained by using the LTspice toolkit.

The signals from the aluminum foil acquired with the experimental setup of figure 5.7 and amplified with the in-house made electronics of figure 5.8 (total gain $=10^{10}$ ), before and during irradiation, are shown in figure 5.10, where the output signals were shifted downwards during irradiation by $11.28,8.36$, and $1.64 \mathrm{~V}$ for stripper currents of 1.6, 1.0, and $0.4 \mu \mathrm{A}$, respectively. Furthermore, the signal transition for a stripper current of $0.15 \mu \mathrm{A}$ was $0.29 \mathrm{~V}$ (not shown).

The corresponding images for the generated scintillation light are shown in figure 5.11. It can be seen clearly that the scintillation light intensity is increasing with stripper current, up to saturation.

\subsection{Assessing beam current using a thin carbon foil}

In order to compare between measuring proton beam current from a PET cyclotron by using a thin aluminum foil and by using a thin carbon foil, the experimental setup which is shown in figure 5.12 has been assembled at ICNAS. The beam current was assessed by measuring the current of secondary electrons liberated (secondary current) from a 5 - $\mu$ m-thick carbon foil and from a $20-\mu \mathrm{m}$-thick aluminum foil, one at a time during two different beam irradiation. 


\section{Chapter 5. On-line measurements of beam current from a PET cyclotron using a thin aluminum foil}
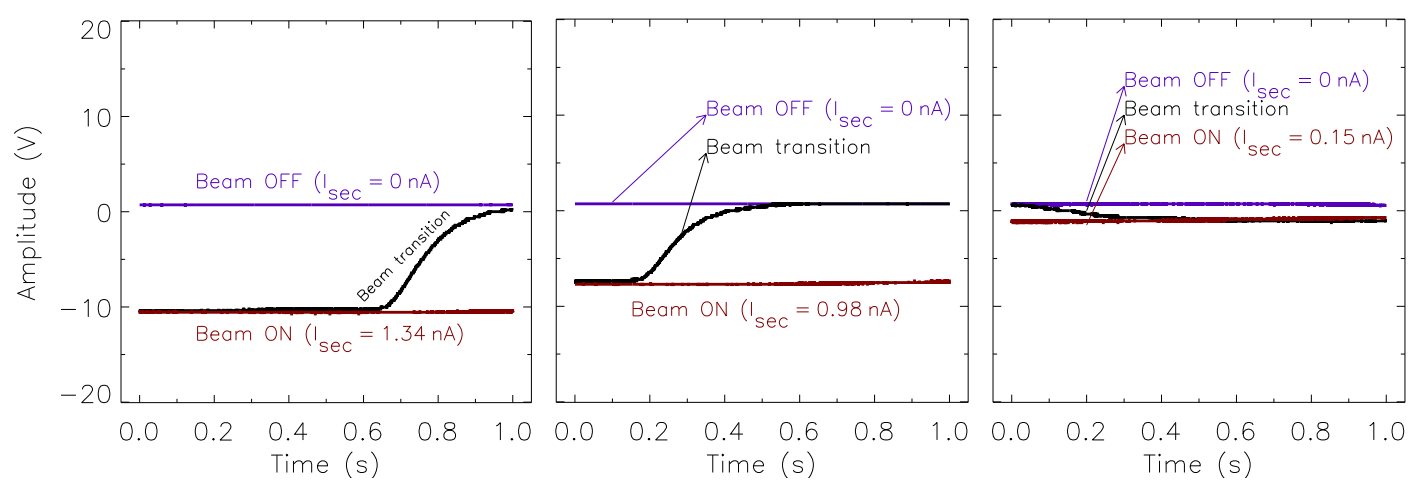

Figure 5.10: Signals from the aluminum foil for the setup of figure 5.7, amplified with the electronics of figure 5.8. The purple signals were acquired when the proton beam was off (before irradiation). The RF (radiofrequency), the magnetic field, and the ion source of ICNAS cyclotron were on. The brown signals were acquired when the proton beam was on (during irradiation). The black signals represent the transition signals between beam on and beam off. The signals were shifted downwards during irradiation by $11.28,8.36$, and $1.64 \mathrm{~V}$, respectively. The corresponding secondary currents were found to be, respectively, 1.34 ( $I_{\text {stripper }}=$ $1.6 \mu \mathrm{A}), 0.98\left(I_{\text {stripper }}=1.0 \mu \mathrm{A}\right)$, and $0.15 \mathrm{nA}\left(I_{\text {stripper }}=0.4 \mu \mathrm{A}\right)$.
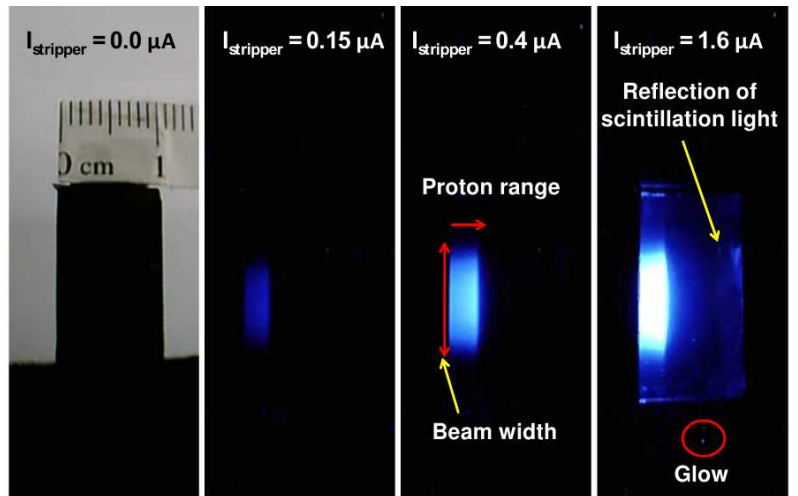

Figure 5.11: Four photographs showing the plastic scintillator of the setup of figure 5.7. The left one was taken before irradiation, while the other three were taken during irradiation at different values of stripper current.

The signals from the foils were measured with a current to voltage converter followed by a voltage amplifier circuit (gain 1000) and an oscilloscope (figure 5.2).

At the beginning, a 1-mm-diameter aluminum collimator was screwed to the 40-cm-long aluminum pipe. After that, a carbon foil was positioned in air after the collimator at distance of about $1 \mathrm{~cm}$ as shown in figure 5.12. Then, the foil was connected to one channel of the Tektronix TD7104 channels (figure [5.2). 

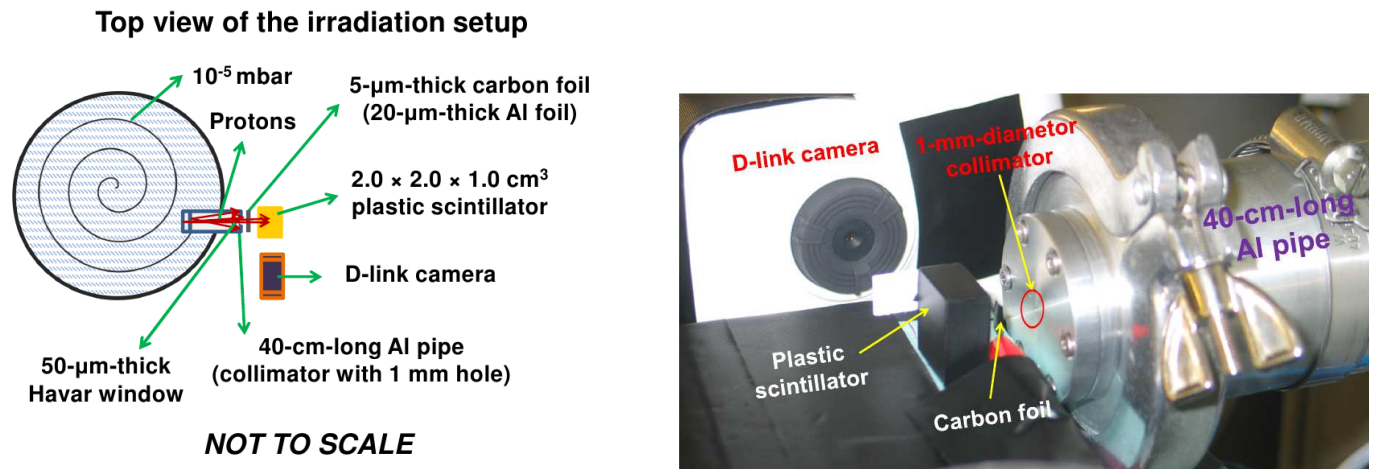

Figure 5.12: Schematic diagram of the experimental setup of the carbon foil experiment (left). It shows a 40-cm-long aluminum pipe, a carbon foil, and a piece of plastic scintillator. Photograph for the same experimental setup (right).

Therefore, we were able to measure the signal from the foil by using the scope. In a second stage, a $20-\mu$ m-thick aluminum foil was used instead of the carbon foil under the same experimental conditions.

The output signals from the carbon and the aluminum foils were shifted downwards during irradiation (beam on) by 1.75 and $0.93 \mathrm{~V}$, respectively. The corresponding secondary currents are, respectively, 1.75 and $0.93 \mathrm{nA}$.

The proton beam energy after the 40-cm-long aluminum pipe was found to be $15.45 \mathrm{MeV}$ 1. The calculated secondary-electron yield (section 5.2) for protons with energy of $15.45 \mathrm{MeV}$ incident on oxidized aluminum and on carbon was found to be 0.238 and 0.431 , respectively. The ratio of the calculated secondary electron yield for the $15.45 \mathrm{MeV}$ proton beam incidents on carbon foil to that on oxidized aluminum is 1.81 . This ratio is consistent with the ratio of the measured secondary current from the carbon foil to that from the oxidized aluminum foil, which is 1.88 .

\subsection{Discussion}

In order to calibrate the signals measured from the aluminum foils, a PicoScope 2203 waveform generator was used to apply a DC signal $(100 \mathrm{mV})$ through a $1 \mathrm{M} \Omega$ load to the readout electronics. This corresponds to an injected current of

\footnotetext{
${ }^{1}$ See section 4.2 .2 , where the material between the beam port and the plastic scintillator is described in detail.
} 


\section{Chapter 5. On-line measurements of beam current from a PET cyclotron using a thin aluminum foil}

$100 \mathrm{nA}$. The mean voltage of the corresponding measured signal was $1 \mathrm{~V}$. In the in-house made electronics (figures 5.2 and 5.8), the current to voltage converter was followed by a voltage amplifier, with an amplification factor of $10^{3}$ (i.e., total gain $=10^{10}$ ). Therefore, the relation between the mean voltage of a signal being read and the secondary current from a given foil is given by equation 5.6. By following the same procedure, the calibration curve of figure 5.13 was obtained. The PicoScope 2203 was used to inject several values of DC signals through a load of $10.22 \mathrm{M} \Omega$, which therefore correspond to the injected currents shown in the $\mathrm{y}$-axis of figure 5.13 .

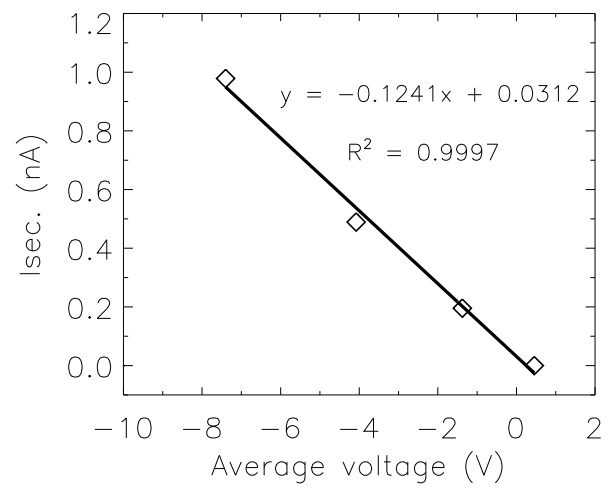

Figure 5.13: Calibration curve for secondary current measurements with a thin aluminum foil. Isec. is the injected secondary current, with the average voltage being the signal read at the oscilloscope.

$$
\frac{\Delta V}{I_{\text {sec. }}}=-\frac{1 \text { volt }}{100 \mathrm{pA}}
$$

In equation 5.6, $\Delta V$ is the mean voltage of a given signal (beam on) without offset i.e., the mean voltage of a given signal (beam on) minus the mean voltage of the same signal (beam off). $I_{\text {sec. }}$ for a given foil is then the measured secondary current.

The proton beam energy after the 40-cm-long aluminum pipe was found to be $15.45 \mathrm{MeV}$. The stopping power for $15.45 \mathrm{-MeV}$ protons in aluminum and in oxidized aluminum are $6.546 \mathrm{MeV} / \mathrm{mm}$ (i.e., $d E / d x=6.546 \times 10^{7} \mathrm{eV} / \mathrm{cm}$ ) and $10.31 \mathrm{MeV} / \mathrm{mm}$, respectively. Therefore, the calculated secondary-electron yield (equation 5.4) for protons with energy of $15.45 \mathrm{MeV}$ incident on oxidized aluminum $\left(Y_{\mathrm{Al}_{2} \mathrm{O}_{3}}=2.25 \cdot Y_{\mathrm{Al}}\right)$ was found to be 0.238 .

The beam current $\left(I_{\text {beam }}\right)$ at the position of the first foil in the stacked target experiment (figure 5.1) was calculated by substituting the measured secondary 
current $\left(I_{\text {sec. }}=188 \mathrm{pA}\right)$ and the calculated secondary-electron yield $\left(Y_{\mathrm{Al}_{2} \mathrm{O}_{3}}=\right.$ 0.238) in the so-called Sternglass formula 5.7 [Forck, 2011]:

$$
I_{\text {sec. }}=Y_{A l} \cdot I_{\text {beam }}
$$

As a result, the calculated beam current is $0.8 \mathrm{nA}$.

This beam current of $0.8 \mathrm{nA}$ corresponds to $5 \times 10^{9}$ protons/s. Consequently, the flux density $\phi$ of the protons from ICNAS cyclotron on a selected region of a target positioned in-air after the $1-\mathrm{mm}$ diameter collimator is given by equation 6.4

$$
\phi\left[\text { protons } /\left(\mathrm{s} . \mathrm{cm}^{2}\right)\right]=\frac{5 \times 10^{9}[\text { protons } / \mathrm{s}]}{\pi r^{2}\left[\mathrm{~cm}^{2}\right]}
$$

where $r$ is the radius of the irradiated area $(r=0.5 \mathrm{~mm}$ (figure 5.1) ). As a result, the calculated flux density is about $6.4 \times 10^{11}$ protons $/\left(\mathrm{s} \mathrm{.} \mathrm{cm}^{2}\right)$.

The relationship between the dose rate $D R$ and the flux density $\phi$ is given by equation 4.1, where $\rho$, in this case, is the density of oxidized aluminum $\left(\rho_{\mathrm{Al}_{2} \mathrm{O}_{3}}=\right.$ $\left.3.97 \mathrm{~g} / \mathrm{cm}^{3}\right)$. The calculated $D R$ in the plateau region in the stacked target experiment (figure 5.1) is about $3 \mathrm{kGy} / \mathrm{s}$.

In respect to the experiments carried out at the end of the 2.4-m-long aluminum pipe (figure 5.7), the proton beam current was measured on-line by using one single thin aluminum foil, with the secondary currents measured by using the calibration curve of figure 5.13. Several measured and calculated values are summarized in table 5.1. These values correspond to the 8-mm-diameter collimator. The results were obtained for 1.6, 1.0, 0.4, and $0.15 \mu \mathrm{A}$ stripper currents.

The energy of the proton beam at the position of the thin aluminum foil without any material between the beam exit and the aluminum foil (figure 5.7) was assessed by measuring its range in the plastic scintillator (figure 5.11). The middle image in figure 5.11 was analyzed with proper software in order to obtain the range $(2.5 \mathrm{~mm})$. Then, the energy of the protons was assessed by using the SRIM/TRIM simulation toolkit. As a result, the energy of proton beam at the position of the thin aluminum foil is $15.2 \mathrm{MeV}$. The stopping power for the 15.2$\mathrm{MeV}$ protons in aluminum and in oxidized aluminum are $6.632 \mathrm{MeV} / \mathrm{mm}$ (i.e., $d E / d x=6.632 \mathrm{keV} / \mu \mathrm{m}$ ) and $10.44 \mathrm{MeV} / \mathrm{mm}$, respectively.

Table 5.1 shows that when the stripper current is decreased by a factor 11 between the first and the last lines, the corresponding dose rate is decreased by a factor of 1675 . This is because, for obtaining smaller currents, the proton beam had to be defocused at the 5 - $\mu \mathrm{m}$-thick stripper foil by means of the applied 


\section{Chapter 5. On-line measurements of beam current from a PET cyclotron using a thin aluminum foil}

Table 5.1: Measured and calculated values from the aluminum foil experiment. Signal transition, secondary current, beam current, and dose rate @ plateau are listed for several values of stripper current for the setup of figure 5.7 .

\begin{tabular}{|c|c|c|c|c|}
\hline \multicolumn{3}{|c|}{ Measured } & \multicolumn{2}{|r|}{ Calculated } \\
\hline $\begin{array}{c}\text { Stripper current } \\
(\mu \mathrm{A})\end{array}$ & $\begin{array}{c}\text { Signal transition } \\
(\mathrm{V})\end{array}$ & $\begin{array}{l}I_{\text {sec. }} \\
(\mathrm{nA})\end{array}$ & $\begin{array}{l}I_{\text {beam }} \\
(\mathrm{nA})\end{array}$ & $\begin{array}{c}\text { Dose rate @ plateau } \\
(\mathrm{Gy} / \mathrm{s})\end{array}$ \\
\hline 1.6 & 11.28 & 1.34 & 5.54 & 289.83 \\
\hline 1.0 & 8.36 & 0.98 & 4.05 & 211.97 \\
\hline 0.4 & 1.64 & 0.15 & 0.62 & 32.44 \\
\hline 0.15 & 0.29 & 0.0008 & 0.0033 & 0.173 \\
\hline
\end{tabular}

magnetic field. Consequently, only a much smaller fraction of all the accelerated protons of the resultant broader beam is bent out in a way to reach the very end of the beamline, with the rest of the proton beam being stopped along the way.

Figure 5.14 shows that measuring the secondary current from a thin aluminum foil positioned in the beam path allows for the dose rate (and total dose) to be assessed for the cyclotron proton beams. This technique is based on measuring secondary electrons liberated when the protons traverse a thin aluminum foil (20$\mu \mathrm{m}$-thick). Since the aluminum foil is thin, the lost energy from the protons when they traverse through the foil is negligible when compared with the nominal beam energy $(\sim 0.116 \mathrm{MeV}$ vs. $18 \mathrm{MeV})$. In addition, the effect of the aluminum foil on the proton direction is negligible. As a result, the method allows for proton beam currents to be measured with minimal proton energy loss and direction change.

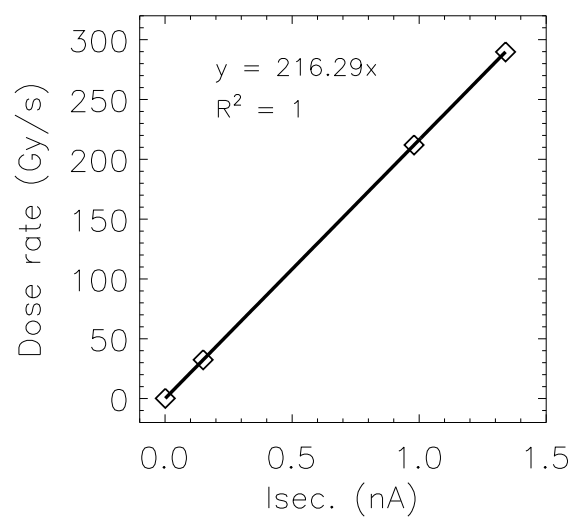

Figure 5.14: Calculated dose rate as a function of the measured secondary current. The linearity between both values can be seen clearly. 


\subsection{Summary and outlook}

We measured the Bragg peak of the protons from a PET cyclotron using a stacked target consisting of several aluminum foils interleaved with polyethylene sheets, readout by in-house made transimpedance electronics. The measured Bragg peak is consistent with simulations performed using the SRIM/TRIM simulation toolkit. Furthermore, we report on experimental results aiming at measuring proton beam currents down to $10 \mathrm{pA}$ using a thin aluminum foil (20- $\mu$ m-thick). The aluminum was chosen for this task because it is radiation hard, it has low density and low radiation activity, and finally because it is easily available at negligible cost. This method allows for calculating the dose delivered to a target during an irradiation with high efficiency, and with minimal proton energy loss and scattering. This technique can be used for measuring beam currents from a PET cyclotron during target irradiation, thus allowing the control of the dose delivered to a target. 
Chapter 5. On-line measurements of beam current from a PET cyclotron using a thin aluminum foil 


\section{Chapter 6}

\section{PET cyclotron based irradiation setup for proton radiobiology and radiophysiology}

\subsection{Motivation}

The nominal energy of the proton beam from ICNAS cyclotron, at the University of Coimbra, is $18 \mathrm{MeV}$. This energy corresponds to a range of $3.45 \mathrm{~mm}$ in water [Ziegler, 2013], therefore the transmission detector used to measure the proton beam current has to be thin enough to minimize proton energy loss and direction change. A possible solution is the use of a thin aluminum foil (20- $\mu$ m-thick) positioned in the beam path. This foil allows for the dose rate (and total dose) to be assessed for the cyclotron proton beam. The signal from the $20-\mu$ m-thick aluminum transmission foil is readout by in-house made transimpedance electronics. This method is based on measuring the secondary electrons liberated from the thin aluminum foil when protons traverse it 1 .

A precise dosimetry is needed for the implementation of radiobiology, radiophysiology, and other dosimetric studies with proton beams. For that, we describe in this chapter the design, characterization, calibration, and validation of an outof-yoke irradiation setup that was assembled and installed at the PET-dedicated cyclotron at the University of Coimbra. This monitoring system was adjusted to the special characteristics of a proton beam from a PET cyclotron. The system

\footnotetext{
${ }^{1}$ See chapter 5
} 
is intended to be applicable to quasi-static proton beams and able to measure total dose as well as dose rate every second, which makes it suitable for real-time target dosimetry. It could be very useful for a number of different future developments and applications of proton beams. To the best of our knowledge this is the first time that a radiobiological irradiation setup was implemented at a PET-dedicated proton cyclotron.

\subsection{Developments for an out-of-yoke irradiation setup}

\subsubsection{Out-of-yoke chopped beam}

In a first stage the proton beam was brought outside the yoke of the PET cyclotron (ICNAS cyclotron) using a 40-cm-long aluminum pipe (figure 6.1). The dose rate at this stage was assessed to be of the order of $3 \mathrm{kGy} / \mathrm{s}$ for minimum cyclotron currents 1. In order to decrease this too high dose rate value, a 2-m-long aluminum pipe was further attached to the first pipe (figure 6.1). Simulations with Geant4 and experimental data 2 show that the beam disperses at a $50-\mu$ m-thick $\operatorname{Havar}^{\circledR}$ foil positioned at the end of the first pipe, reaching the end of the 2-m-long pipe with a proton flux decreased by two to three orders of magnitude, depending on cyclotron current settings.

In addition to the first 50 - $\mu$ m-thick Havar ${ }^{\circledR}$ window (30 mm in diameter), which is located at the end of the 40-cm-long aluminum pipe, a second Havar ${ }^{\circledR}$ window was positioned at the end of the 2-m-long aluminum pipe (figure 6.1). This window separates the vacuum inside the 2 -m-long aluminum pipe $\left(10^{-2} \mathrm{mbar}\right)$ from the atmosphere in the bunker. Right after the second Havar ${ }^{\circledR}$ window, an aluminum collimator with variable diameter $(23 \mathrm{~mm}$ down to $0.5 \mathrm{~mm})$ can be fixed to the 2-m-long aluminum pipe.

The measured dose rate at the end of the long pipe ranges from $1 \mathrm{kGy} / \mathrm{s}$ down to $10 \mathrm{~Gy} / \mathrm{s}$. These dose rate values are too high for radiobiology studies controlled by a shutter that responds on the order of fractions of a second i.e., irradiation of tens and hundreds of mGy would not be possible. This excessive dose rate is overcome by using a rotating disk with a slit of $0.5-\mathrm{mm}$ width located at a disk

\footnotetext{
${ }^{1}$ See chapter 5

${ }^{2}$ See section 3.3 .
} 
radius of $150-\mathrm{mm}$ (figure 6.1) allowing for decreasing target dose rates by a factor of $0.5 \mathrm{~mm} /(2 \pi 150 \mathrm{~mm})=5 \times 10^{-4}$ (figure 6.2). As a result, the target dose rate after the rotating slit is ranging from $500 \mathrm{mGy} / \mathrm{s}$ down to $5 \mathrm{mGy} / \mathrm{s}$ (figure 6.1). The slit was implemented with a width of $0.5 \mathrm{~mm}$ (reduction factor of $5 \times 10^{-4}$ ) and can be adjusted up to $3 \mathrm{~mm}$ (reduction factor of $3 \times 10^{-3}$ ). The rotating disk was fixed to an aluminum support and coupled to a geared DC motor, which is controlled using a four quadrant, linear output motor driver.

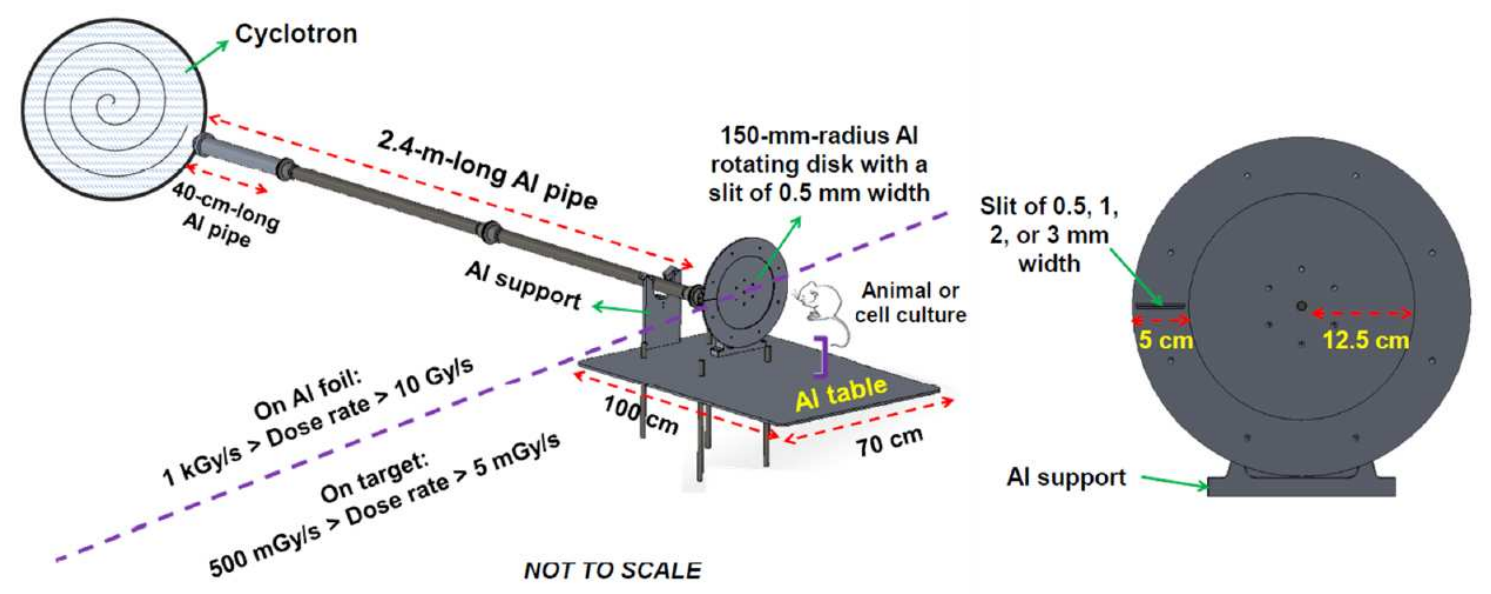

Figure 6.1: Schematic diagram of the irradiation setup. It shows a PET cyclotron, a 2.4-m-long aluminum pipe, an aluminum table $\left(70 \times 100-\mathrm{cm}^{2}\right.$-surface area) and a 300-mm-diameter rotating disk with a slit of variable width. In addition, it shows dose rate values before and after the rotating slit.

\subsubsection{Hardware and software for dose and dose rate mea- surements}

Figure 6.3 shows the out-of-yoke irradiation setup that has been assembled at ICNAS. The 2.4-m-long aluminum pipe permits the proton beam produced in the cyclotron to reach a target at its end, where the beam enters through a 23-mm-diameter hole. The $18-\mathrm{MeV}$ proton beam exits the cyclotron through the first Havar ${ }^{\circledR}$ window, travels along the pipe and passes through the second Havar ${ }^{\circledR}$ window. The proton beam that passes through the 23 -mm diameter hole positioned after the second Havar window trespasses a $20-\mu \mathrm{m}$-thick aluminum foil positioned in air immediately after this collimator. The beam current on target is assessed by measuring the current of the secondary electrons liberated (secondary current) from this thin aluminum foil (figure 6.3, top left). 


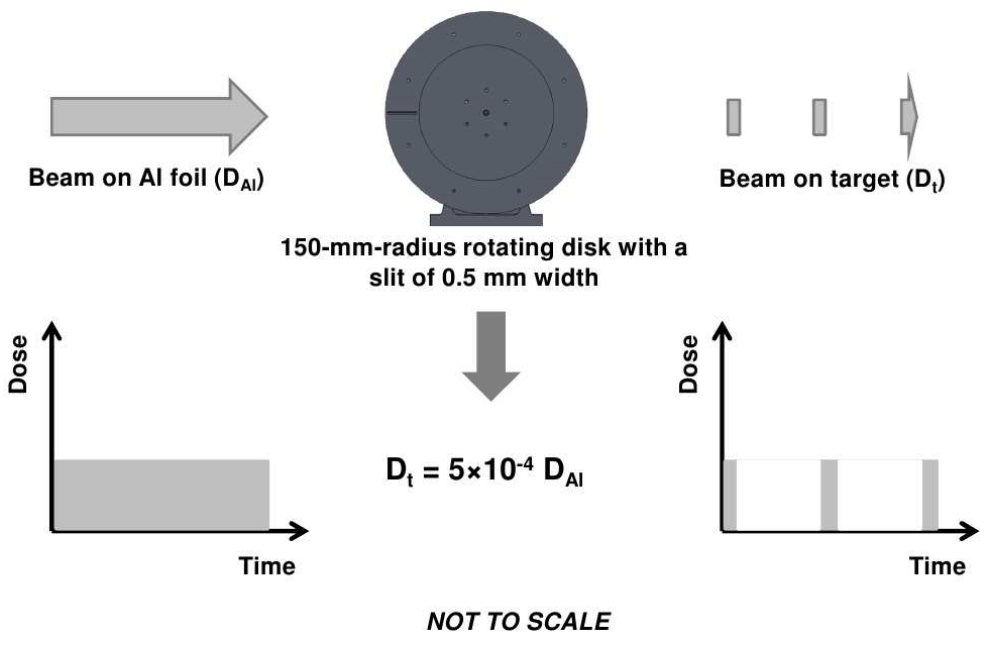

Figure 6.2: Schematic view of the beam duty cycle (right images) resulting from a $3 \mathrm{~Hz}$ chopper (middle image) for high-intensity proton beams from a PET cyclotron.

The 23-mm-diameter hole is covered at the outside by a piece of black foil in order to prevent the secondary electrons liberated from the aluminum collimator and the second Havar ${ }^{\circledR}$, window from reaching the aluminum foil. The aluminum foil was fixed at the outer surface of the black foil in alignment with the 23-mmdiameter hole. Another piece of black foil is used to cover the outer surface of the thin aluminum foil in order to prevent the secondary electrons liberated from the rotating disk from reaching it. The aluminum foil is covered by a sheet of aluminum (figure 6.3, top right) to shield it from noise signals of $3 \mathrm{~Hz}$ induced by the rotating slit.

The signal from the aluminum foil is amplified and connected via a $25-\mathrm{m}-$ long BNC cable to a digital oscilloscope (PicoScope 2203) positioned outside the bunker and connected by USB to a laptop on Linux. Another 25-m-long BNC cable is connected to the thin aluminum foil through a $110 \mathrm{M} \Omega$ resistor from one side and biased by a DC voltage supplied by the PicoScope 2203 from the other side for the purpose of current calibration. A target can be positioned in air next to the rotating disk perpendicularly to the proton beam axis and in alignment with the 23-mm-diameter hole. A computer-controlled shutter positioned at a distance of $1 \mathrm{~cm}$ after the rotating slit (figure 6.3, top) allows to control the dose on target.

The thin aluminum foil is readout by the in-house made electronics shown in figure 6.4. The signals from the thin aluminum foil go through a current to voltage converter, with amplification factor of $10^{7} \mathrm{~V} / \mathrm{A}$, followed by a low pass filter. 
6.2. Developments for an out-of-yoke irradiation setup
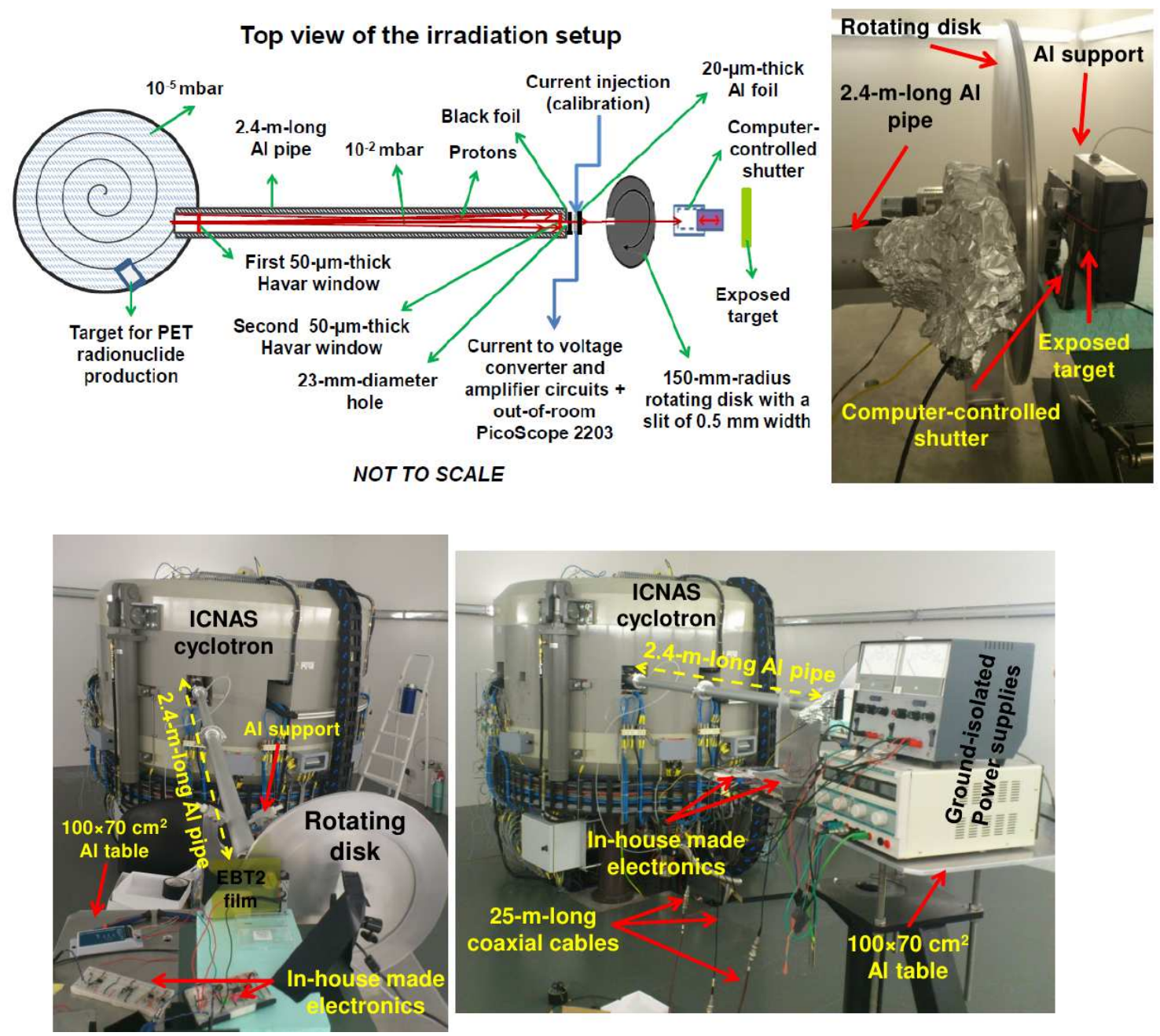

Figure 6.3: Schematic diagram of the out-of-yoke irradiation setup. It shows a 2.4-m-long aluminum pipe with a 23-mm-diameter hole at its end, an aluminum foil, a 300-mm-diameter rotating disk with a slit of 0.5 -mm width, a computercontrolled shutter, and a target positioned in air perpendicularly to the proton beam path. The three photographs (top right and bottom) show a detail of the area where the irradiation setup was assembled.

The filtered signals are amplified again using a voltage amplifier with adjustable gain between $10^{2}$ to $10^{3}$. The amplified signals are filtered again using an $11^{\text {th }}$ order Chebyshev low pass filter in order to attenuate undesired $50 \mathrm{~Hz}$ frequency noise that would otherwise decrease the voltage dynamic range and increase the error percentage in the measurements. The $11^{\text {th }}$ order Chebyshev low pass filter is shown in figure 6.4 between $V_{\text {out } 1}$ and $V_{\text {out }}$. Finally, the resulting signals are measured using a PicoScope 2203 oscilloscope, which is connected to a laptop on 


\section{Chapter 6. PET cyclotron based irradiation setup for proton radiobiology and radiophysiology}

Linux running an in-house developed $\mathrm{C} / \mathrm{C}++$ program. A voltage offset adjustment is shown in figure 6.4 at the negative input of the second amplifier. This offset is used to balance the output voltage within the range of $\pm 10 \mathrm{~V}$, where the $1 \mathrm{M} \Omega$ resistor injects a current which produces a voltage drop across the $1 \mathrm{k} \Omega$ resistor, and therefore changes the voltage offset. The output signals are adapted using a high precision potentiometer $(50 \mathrm{k} \Omega)$ of the voltage offset adjustment circuit. The main terminal of the potentiometer is connected to the $1 \mathrm{M} \Omega$ resistor, whereas one of the remaining two terminals is connected to the ground and the other one is connected to a power supply through a voltage regulator (KA7805). The latter was necessary in order to provide a rejection of power supply oscillations in the range of tens of $\mathrm{mV}$ that would otherwise disturb the precision of the secondary current measurements. The in-house developed electronics was installed inside a grounded aluminum box for shielding the radiofrequency (42 $\mathrm{MHz}$ ) of the cyclotron.

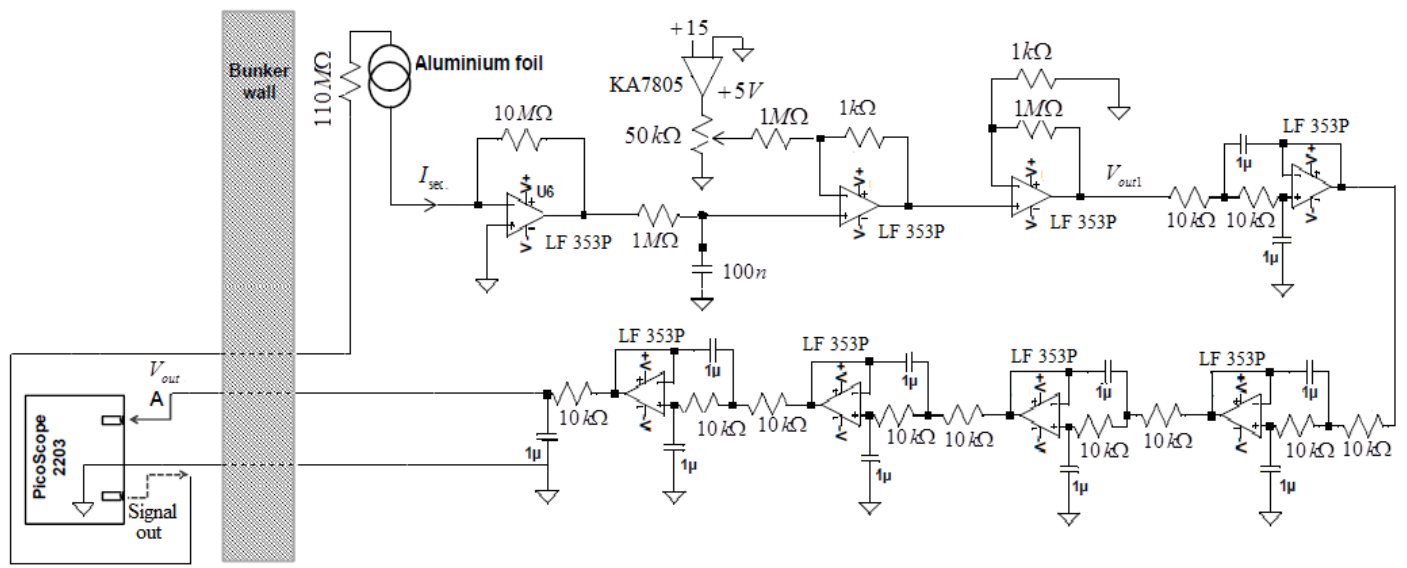

Figure 6.4: Schematic diagram of an in-house made transimpedance electronics, with total gain of $10^{10}$, that is used to readout the signals from the thin aluminum foil. It shows a current to voltage converter followed by a low pass filter, a voltage offset adjustment, a voltage amplifier circuit, an $11^{\text {th }}$ order Chebyshev low pass filter and an oscilloscope (PicoScope 2203).

In order to calibrate the signals measured from the thin aluminum foil, the built-in waveform generator was used to inject several DC signals $V_{D C}$ through a $110 \mathrm{M} \Omega$ load to the readout electronics as shown in figure 6.4 (total gain = $10^{10}$ ). This corresponds to an injected current $I_{\text {injected }}$ given by Ohm's law (equation 6.1).

$$
I_{\text {injected }}[n A]=\frac{V_{D C} \operatorname{signal}[m V]}{110[M \Omega]}
$$




\subsection{Developments for an out-of-yoke irradiation setup}

A console-mode program in $\mathrm{C} / \mathrm{C}++$ has been written to read and analyze the signals from the $20-\mu \mathrm{m}$-thick aluminum foil. This program is able to collect a stream of data in sampling intervals of $960 \mathrm{~ns}$ each, using free trigger mode. Every second, the program calculates the average voltage of all measured signals. The corresponding secondary-electron current $I_{\text {sec. }}$ is calculated using a calibration equation. In addition, proton beam current, flux, flux density, and dose rate are also assessed every second. Furthermore, the accumulated dose during target irradiation is also computed.

The beam current $I_{\text {beam }}$ at the position of the thin aluminum foil is calculated by substituting the measured secondary current $I_{\text {sec. }}$ and the calculated secondary-electron yield $Y_{\mathrm{Al}_{2} \mathrm{O}_{3}} 1$ in the so-called Sternglass formula 6.2 [Forck, 2011]:

$$
I_{\text {beam }}=\frac{I_{\text {sec. }}}{Y_{\mathrm{Al}_{2} \mathrm{O}_{3}}}
$$

the corresponding flux is calculated using equation (6.3).

$$
\text { Flux }[\text { protons } / s]=\frac{I_{\text {beam }}[A]}{e^{-} \text {charge }[C]},
$$

where $e^{-}$charge is the electron charge $\left(1.6 \times 10^{-19} C\right)$.

The flux density $\phi$ of the protons from ICNAS cyclotron on a selected region of a target positioned in-air after a collimator at the end of the 2.4-m-long aluminum pipe (figure 6.3) is given by equation 6.4:

$$
\phi\left[\text { protons } /\left(s . \mathrm{cm}^{2}\right)\right]=\frac{f l u x[\text { protons } / \mathrm{s}]}{\pi r^{2}\left[\mathrm{~cm}^{2}\right]},
$$

where $r$ is the radius of the irradiated area.

The relationship between the dose rate $D R$ and the flux density $\phi$ is given by equation 6.5 [Kraft, 2000].

$$
D R[G y / s]=1.6 \times 10^{-9} \cdot \frac{d E}{d x}\left[\frac{k e V}{\mu m}\right] \cdot \phi\left[\frac{1}{s \cdot c m^{2}}\right] \cdot \frac{1}{\rho}\left[\frac{c m^{3}}{g}\right],
$$

$d E / d x$ being the stopping power of the incident protons in the irradiated target and $\rho$ is the density of the irradiated target.

\footnotetext{
${ }^{1}$ See section 5.2 .2
} 
The input voltage range of the PicoScope 2203 waveform generator (i.e., \pm 20 , $\pm 10, \pm 5, \pm 2$, and $\pm 1 \mathrm{~V}$ ) for the used channel has to be selected before running the $\mathrm{C} / \mathrm{C}++$ program (before starting the acquisition process). In order to calibrate the collected ADC (analog-to-digital converter) counts, a PicoScope 2203 waveform generator, a Tektronix TDS7104, a power supply, and a personal computer (PC) were used (figure 6.5, left). The power supply was used in order to inject several values of DC signals. The voltage values of the output signals were confirmed using the Tektronix TDS7104. The corresponding ADC values were measured using the PicoScope 2203 waveform generator via the $\mathrm{C} / \mathrm{C}++$ program. Since the trigger level was set to 1500 ADC counts, the trigger point varies depending on the voltage range. The calibration was performed for a voltage range of $\pm 10 \mathrm{~V}$. The calibration curve (calibration equation) is shown in figure 6.5, right.
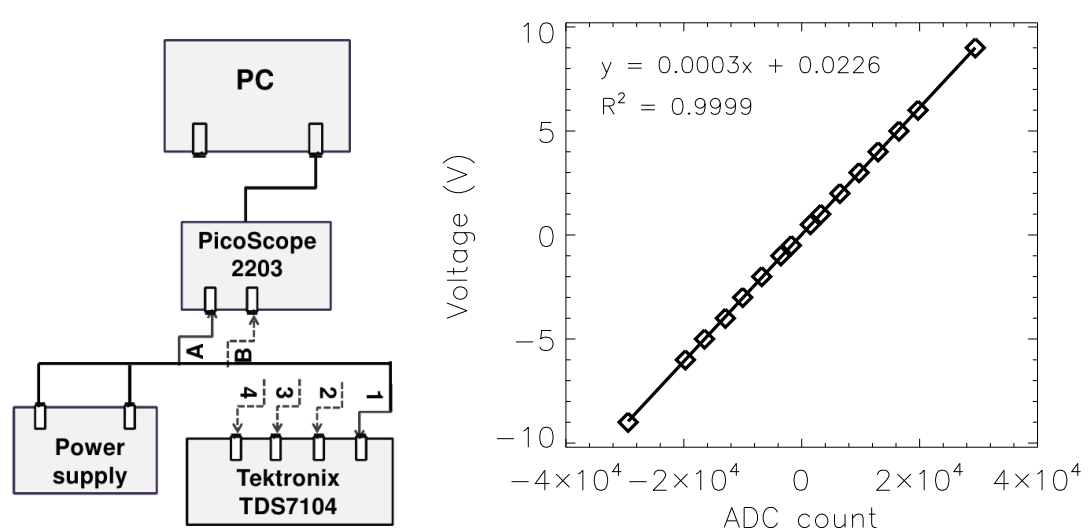

Figure 6.5: Left: Schematic diagram of the experimental setup that was used for converting the measured ADC counts by a PicoScope 2203 waveform generator via a $\mathrm{C} / \mathrm{C}++$ program into volts. It shows the PicoScope 2203, a Tektronix TDS7104, a power supply, and a personal computer (PC). Right: The output calibration curve, converting the measured ADC counts into volts for a voltage range of $\pm 10 \mathrm{~V}$, is shown. The corresponding calibration equation can be seen on the figure.

\subsubsection{Controlled beam exposure via a ground-isolated power system}

In order to develop a proton beam monitoring system at a PET-dedicated cyclotron that can control the dose delivered to a target during irradiation, a shutter which can be controlled using a computer with isolated ground is needed. 


\subsubsection{Beam shutter}

A computer-controlled shutter was installed before the target (figure 6.6). A 4mm-thick $\left(4.5 \times 7.0-\mathrm{cm}^{2}\right.$-front area) aluminum piece was fixed on the outer surface of the animated widget of the shutter. As a result, this piece can be moved via the motor forth and back on the railway of the shutter by delivering the proper voltage from a CAMAC (computer automated measurement and control) system (section 6.2.3.2). The proton beam before target irradiation is stopped on the 4-mm-thick aluminum piece, whereas during irradiation the motor moves this piece away from the beam path, therefore allowing the beam to impinge on the target. The $\mathrm{C} / \mathrm{C}++$ console-mode program insures that, when a prescribed dose is achieved, the aluminum piece is moved back to its original place in the beam path.
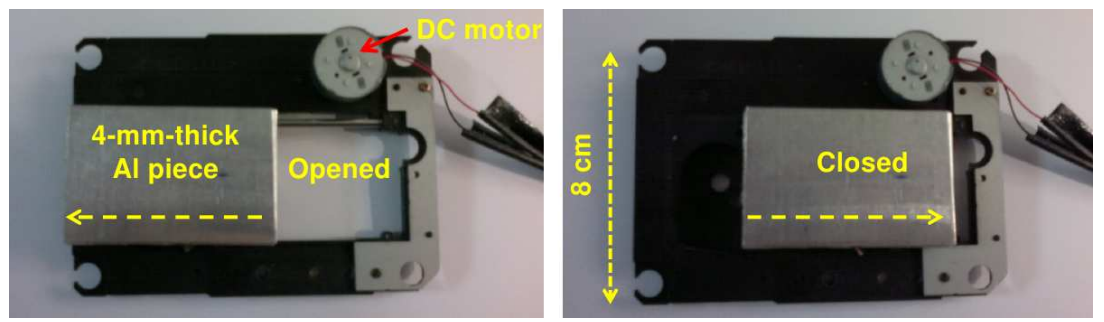

Figure 6.6: Two photographs of a computer-controlled shutter, opened (left) and closed (right). They show a DC motor and a 4-mm-thick aluminum piece.

\subsubsection{Hardware for beam control}

Figure 6.7, top, shows the in-house developed electronics systems for beam readout and control of beam exposure. This figure shows a laptop on Linux with isolated grounds. Consequently, the electronics of the computer-controlled shutter is totally separated from that of the thin aluminum foil that provides beam current information. Thus, the shutter electronics does not disturb the output signals from the thin aluminum foil 1 .

\footnotetext{
${ }^{1} \mathrm{~A}$ frequency-to-voltage converter circuit (appendix C) has been assembled at ICNAS in order to control the beam shutter via a PicoScope 2203 waveform generator. However, experimental results reveal that the in-house developed electronics systems for beam readout and control of beam exposure (the shutter and its related electronics i.e., the frequency-to-voltage converter) have a common ground, where the shutter electronics was introducing some noise to the output signals from the aluminum foil. This issue was solved by controlling the shutter via a CAMAC system and a laptop with isolated grounds.
} 

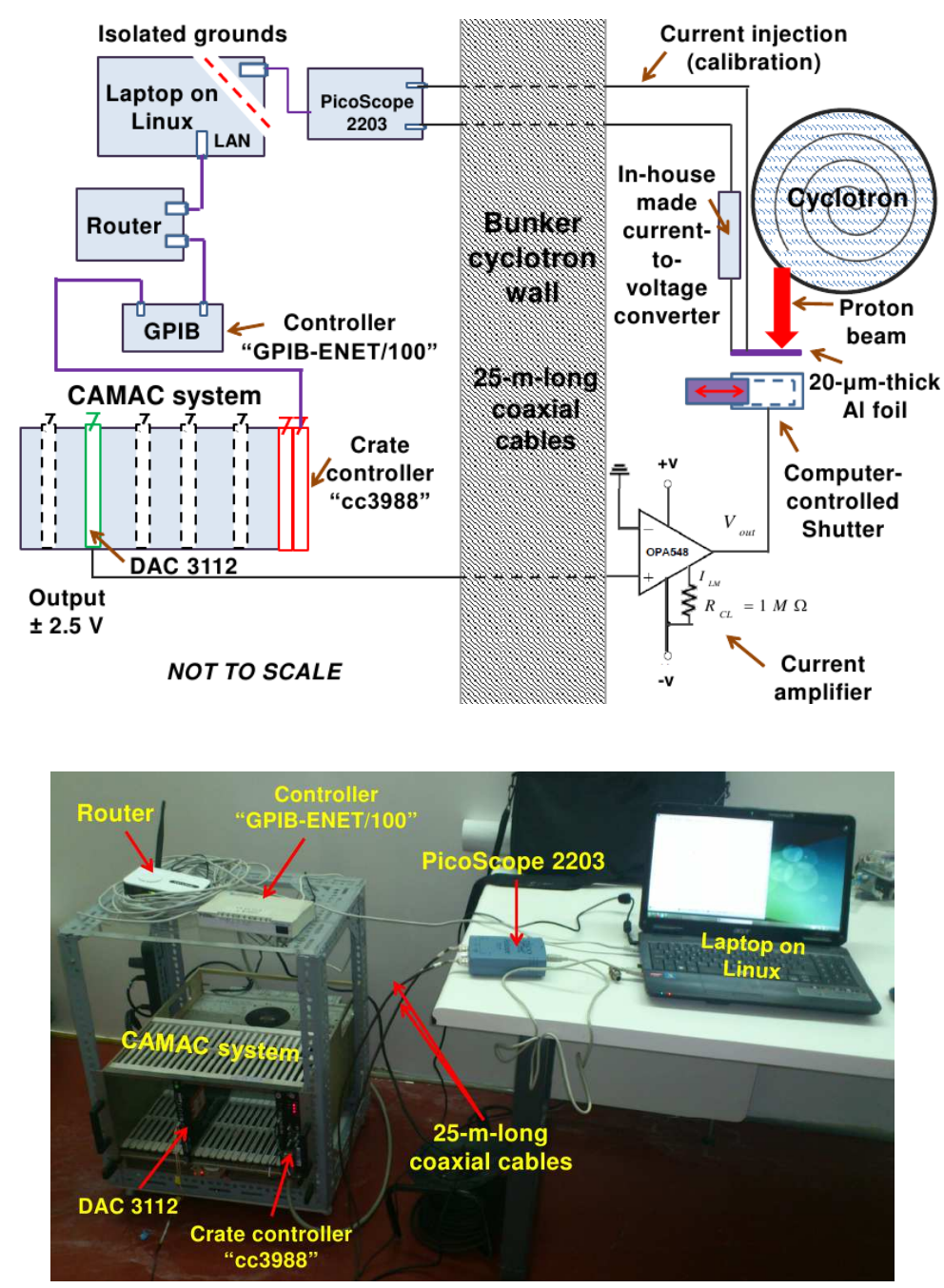

Figure 6.7: Schematic diagram of in-house developed electronics systems for beam readout and control of beam exposure. It shows a laptop on Linux, a router, a controller "GPIB-ENET/100", a crate controller "cc3988", a DAC 3112 module, a current amplifier "OPA548", a computer-controlled shutter and three 25-mlong coaxial cables. In addition, this scheme shows a transmission thin (20$\mu \mathrm{m}$-thick) aluminum foil trespassed by the proton beam and readout by in-house made transimpedance amplifiers, providing online dose information via a $\mathrm{C} / \mathrm{C}++$ program that reads an oscilloscope (PicoScope 2203). The photograph on the bottom shows a detail of the area, outside the bunker of the PET cyclotron, where the modules of the hardware for beam control and the PicoScope 2203 are assembled. 
The laptop on Linux, which is shown in figure 6.7, is part of the hardware for beam control. This laptop is used in order to run a $\mathrm{C} / \mathrm{C}++$ program that sends commands to CAMAC modules to generate a voltage of $\pm 2.5 \mathrm{~V}$ that is applied to the motor of the shutter before, during and after irradiation. A router is used to connect the laptop with a GPIB-ENET/100 (general purpose interface bus/etherNET/100) controller, which allows the laptop to communicate with a crate controller "cc3988". The GPIB-ENET/100 controller communicates with a DAC (digital-to-analog converter) 3112 module via the GPIB crate controller "cc3988". The DAC 3112 module is a single-width CAMAC module for generating eight output voltages. In the CAMAC system installed at ICNAS, channel 4 was adjusted via straps so that the output range of this channel can be changed in the field of $\pm 10 \mathrm{~V}$ at $5 \mathrm{~mA}$ maximum.

The DAC 3112 module is used to generate a voltage of $\pm 2.5 \mathrm{~V}$. Both voltages are necessary for the operation of the shutter in the proper way. Since the driven current from the DAC 3112 module (5 mA maximum) to the shutter motor is not sufficient for the motor to operate, then a high-voltage high-current operational amplifier (OPA548) is used, as shown in figure 6.7, top. The irradiation time on a target is controlled via the developed computer-controlled shutter that responds on the order of fractions of a second.

A time calibration for the whole system has also been performed. For that, a timer was run for $100 \mathrm{~s}$. At the same time, the $\mathrm{C} / \mathrm{C}++$ program was run (all codes and commands that deal with the CAMAC system and with the PicoScope 2203 waveform generator were written previously). As a result, the $100 \mathrm{~s}$ in realtime was found to be $93 \mathrm{~s}$ in system-time i.e., there was a time delay of $7 \mathrm{~s}$ in the developed dosimetry system-time. In order to solve this issue, the number of sampling points in the $\mathrm{C} / \mathrm{C}++$ program was adapted so that the system-time equals the real-time.

\subsection{Results}

\subsubsection{Optimum cyclotron magnetic field for homogenous target irradiation}

Beam homogeneity can be achieved by scanning the magnet current $I_{B}$ with the target shutter closed and the dose rate measurement system on, as shown in figure 6.8. The magnet current is scanned and the corresponding dose rate is 
measured using the developed irradiation system (figure 6.3). Such a scanning process is needed to be performed before target irradiation at a PET-dedicated cyclotron in order to determine the value of the electric current that has to be injected in the cyclotron magnet coils (i.e., magnet current) in order to produce focused protons hence producing a homogenous target dose. This value depends on the daily use of the cyclotron, thus it changes from one day to another. Data evaluation (section 6.3.1.1) reveals that the proton beam is focused at a magnet current that corresponds to the top of the Gaussian curve of figure 6.8, whereas it is defocused at magnet currents that correspond to the skirts of the curve. The measured dose rate using the developed irradiation system can be adapted to a certain value by adjusting the ion source current $I_{\text {source }}$ using the computer system of the cyclotron.
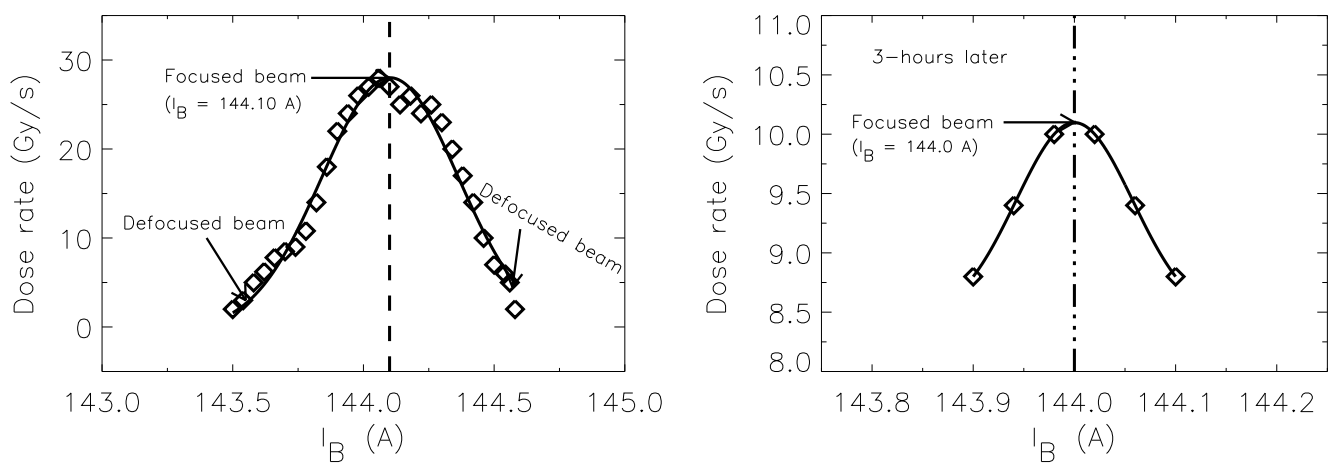

Figure 6.8: Measured dose rate as a function of magnet current $I_{B}$ for two different ion source currents. The dose rate was measured using the developed irradiation system, whereas the magnet current and the ion source current were adjusted using the computer system of the cyclotron. Target irradiation revealed that the proton beam is focused at a magnet current of $144.10 \mathrm{~A}$ (left) and 144.0 A (right), respectively.

\subsubsection{On target beam inhomogeneity with defocused magnetic field}

Two stacked targets, each one consisting of 10 Gafchromic ${ }^{\circledR}$ EBT2 films, were irradiated with focused and defocused beams with a preset dose of $4.2 \mathrm{~Gy}$. The measured signal-to-noise ratio SNR was found to be superior to 1001. The color of the irradiated areas of the EBT2 films changed to black when exposed to ionizing radiation (i.e., proton beam) as shown in figure 6.9. The signals from the thin aluminum foil were acquired with the experimental setup of figure 6.3 and amplified with the in-house made electronics of figure 6.4 $\left(\right.$ total gain $\left.=10^{10}\right)$.

\footnotetext{
${ }^{1}$ The measured signal of several volt oscillated with an amplitude of a few $\mathrm{mV}$ only.
} 


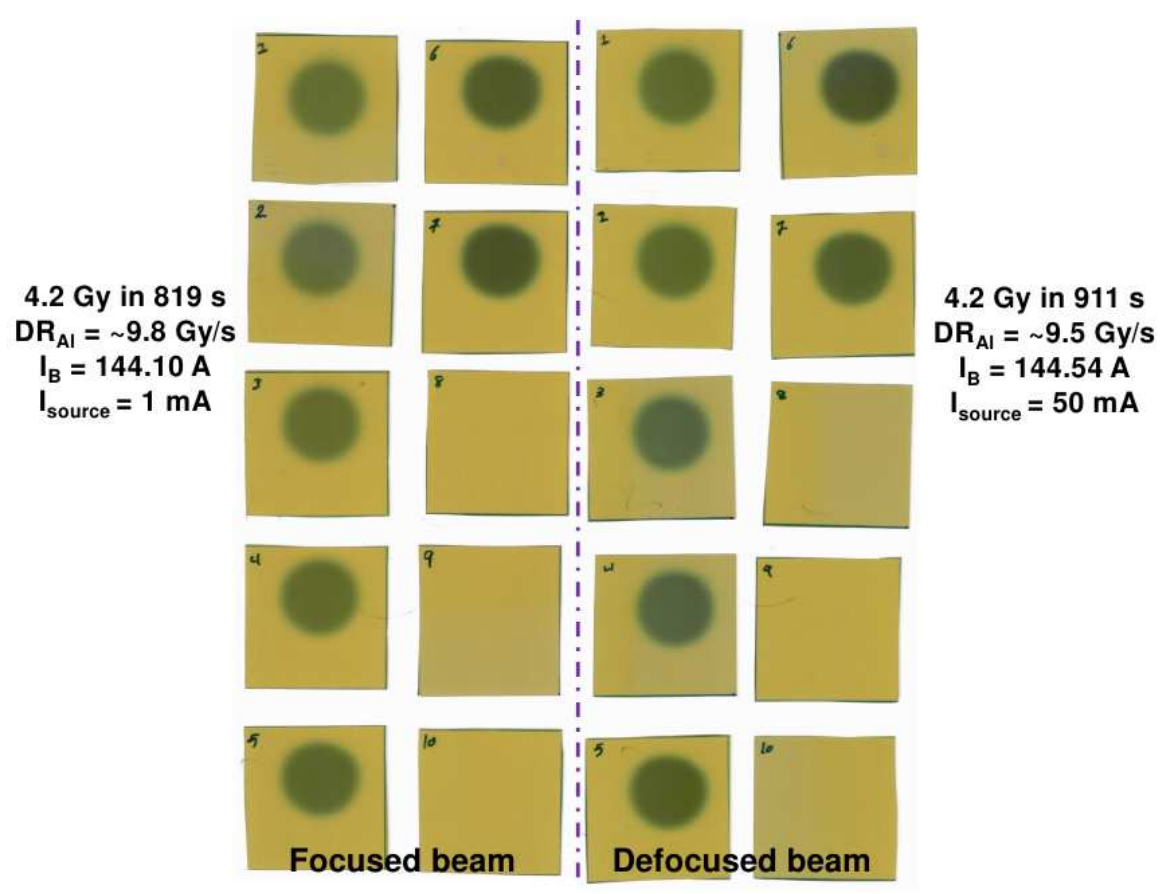

Figure 6.9: Experimental results obtained with two stacked targets for the setup of figure 6.3. The left 10 EBT2 films, each with $285-\mu \mathrm{m}$ thickness, compose the stacked target that was irradiated with focused protons, whereas the right ones compose the stacked target that was irradiated with defocused protons.
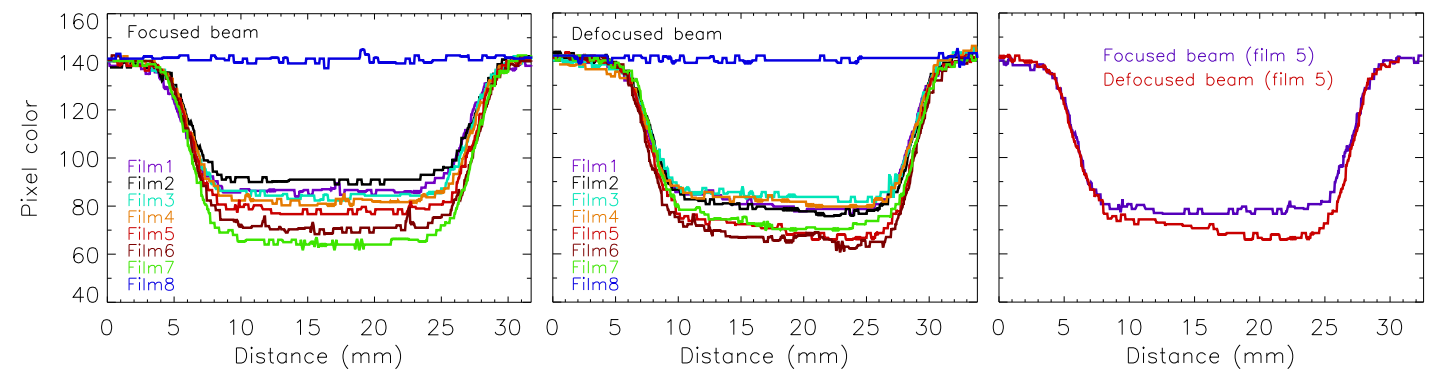

Figure 6.10: Proton beam profiles for the beam spots that were induced by focused (left) and defocused (middle) protons in the first 8 EBT2 films of the two stacked targets (figure 6.9). The right figure shows the proton beam profiles obtained for beam spots at the plateau of the focused (violet) and defocused (brown) beams.

The corresponding on-target beam profiles are shown in figure 6.10, left (focused beam) and middle (defocused beam). These profiles were obtained by scanning the films with a scanner and further processing the resulting images with the software package ImageJ [ImageJ team, 2004]. Figure 6.10, right, shows the beam profiles at the plateau (film 5) of the focused and defocused beams 
from the PET cyclotron. It can be seen that the proton beam profiles of the beam spots that were induced by the focused beam are uniform (i.e., the pixel color oscillates within $\pm 2 \%$ in the middle flat region). However, the profiles of the beam spots that were induced by the defocused beam are asymmetric, they are shifted to the right side (i.e., the pixel color oscillates within $\pm 6 \%$ in respect to the middle region).

Figure 6.11, right, shows a 3D surface plot of a homogenous proton beam spot (figure 6.11, left). The beam spot was obtained after irradiating an EBT2 film with focused protons using the developed irradiation setup at ICNAS. As it can be observed the irradiation was quite uniform i.e., the fluence distribution is uniform to within $\pm 2 \%$ over a central field of $18 \mathrm{~mm}$ in diameter.
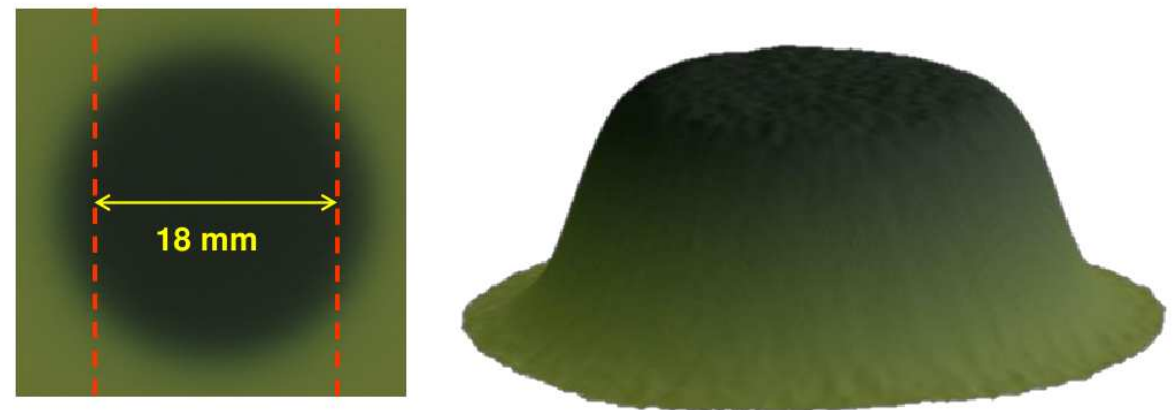

Figure 6.11: Left: A scan copy of an irradiated EBT2 film with focused protons at ICNAS. Right: 3D surface plot of the beam spot obtained with the software package ImageJ [ImageJ team, 2004]. It can be seen that the central region is quite uniform (the pixel color oscillates within $\pm 2 \%$ in the middle flat region), hence the dose in this region is quite homogeneous.

A rectangular slit of $0.5 \mathrm{~mm}$ width, and $5 \mathrm{~cm}$ length along the wheel diameter, is used as a beam chopper (figure 6.1). The distance of the center of the slit to the rotation axis is $150 \mathrm{~mm}$. Then, for beam spots of $18 \mathrm{~mm}$ diameter, there is a gradient of the transmitted fluence for a uniform flux beam of $\pm 9 / 150= \pm 6 \%$ along the diametric direction of the wheel. However, the gradient of the transmitted beam fluence caused by means of the rotating disk is not the dominant factor. In this case, there is another factor playing a major role, which is the magnetic field inside the cyclotron (section 6.3.1). Figure 6.12 shows proton beam profiles of two beam spots, which were induced by defocused beams. The proton beam profile which is shown in figure 6.12, left, corresponds to a beam spot that was induced by defocused protons using a magnet current of $143.6 \mathrm{~A}$, which corresponds to the left skirt of the Gaussian curve of figure 6.8, left. On the other hand, the proton beam profile which is shown in figure 6.12, right, corresponds to a beam 
spot that was induced by defocused protons using a magnet current of $144.54 \mathrm{~A}$, which corresponds to the right skirt of the Gaussian curve. Therefore, the proton beam profile asymmetries, which are induced by defocused beams, might be shifted to the left or to the right, depending on the magnet current that is used to produce the defocused beam. Hence, optimum magnet current must be taken from the center of the Gaussian-shaped curve, producing a homogenous dose on target.
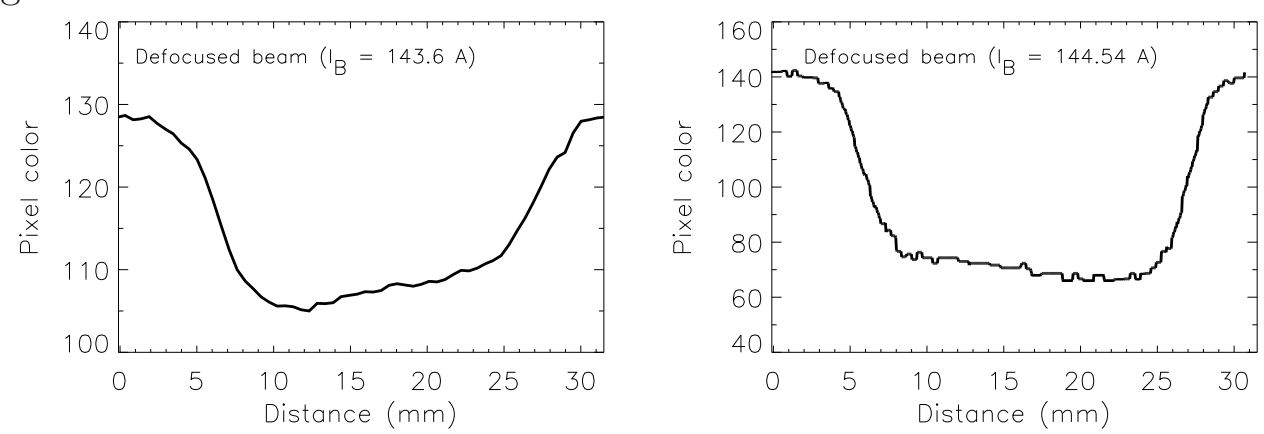

Figure 6.12: Proton beam profiles obtained for two beam spots at the plateau of defocused beams. It can be seen that both beam profiles are asymmetric. The left beam profile is shifted to the left $\left(I_{B}=143.6 \mathrm{~A}\right)$, whereas the right one is shifted to the right $\left(I_{B}=144.54 \mathrm{~A}\right)$.

The setup shown in figure 6.3 allows for measuring the Bragg peak of the proton beam from the PET cyclotron. The measured Bragg peaks for the focused and defocused beams are shown in figure 6.13. For proton dose verification, the under-response in the Bragg peak region, which can be seen in the measured Bragg peaks (figure 6.13), has to be taken into account. For EBT2 films, Reinhardt et al. [2012] reported a dosimetric under-response of about 3\% in the Bragg peak for proton energy less than $40 \mathrm{MeV}$ and up to $17 \%$ for proton energy of $4 \mathrm{MeV}$.

Due to the material present in front of the stack of EBT2 films, the measured beam range in the stack of consecutive EBT2 films was found to be $1.8 \mathrm{~mm}$, with a spatial resolution of $0.14 \mathrm{~mm}$ given by half the width of each EBT2 film (figure 6.14). This range corresponds to a beam energy of $13.75 \pm 0.55 \mathrm{MeV}$. This energy was obtained for the proton beam at the entrance surface of the stacked target using the SRIM/TRIM simulation toolkit [Ziegler, 2013]. The stopping power for $13.75 \mathrm{-MeV}$ protons in aluminum is $7.171 \mathrm{MeV} / \mathrm{mm}$ (i.e., $d E / d x=$ $\left.7.171 \times 10^{7} \mathrm{eV} / \mathrm{cm}\right)$. Therefore, the calculated secondary-electron yield 1 for protons with energy of $13.75 \mathrm{MeV}$ incident on oxidized aluminum $\left(Y_{\mathrm{Al}_{2} \mathrm{O}_{3}}=2.25 \cdot Y_{\mathrm{Al}}\right)$ was found to be 0.262 .

\footnotetext{
${ }^{1}$ See section 5.2 .2
} 


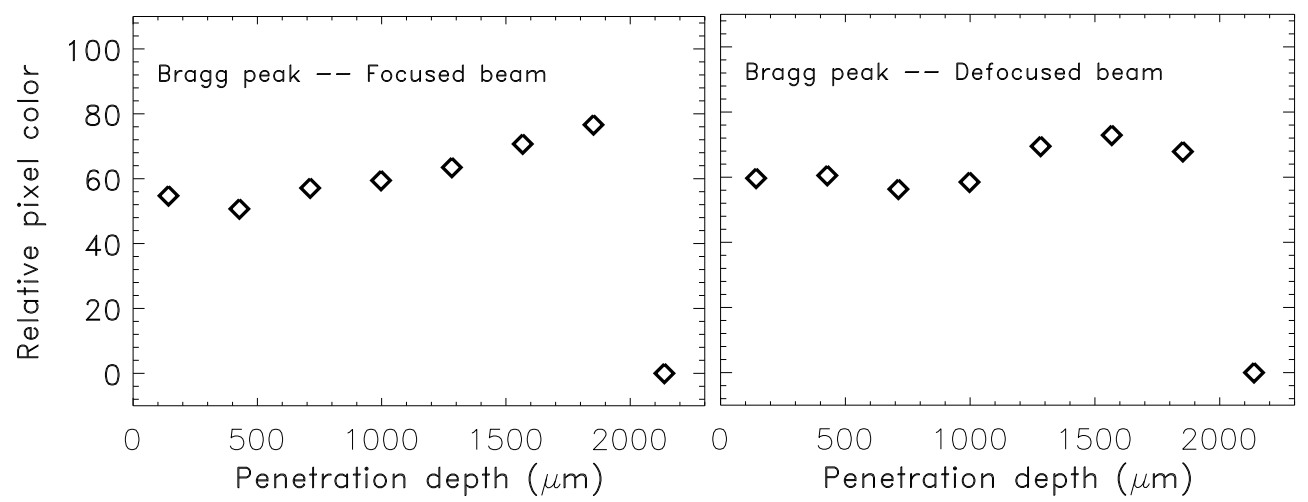

Figure 6.13: Measured Bragg peak for focused (left) and defocused (right) proton beams from the PET cyclotron for the setup of figure 6.3. The dosimetric underresponse in the Bragg peak regions can be seen.

Stacked target consisting of 9 EBT2 films

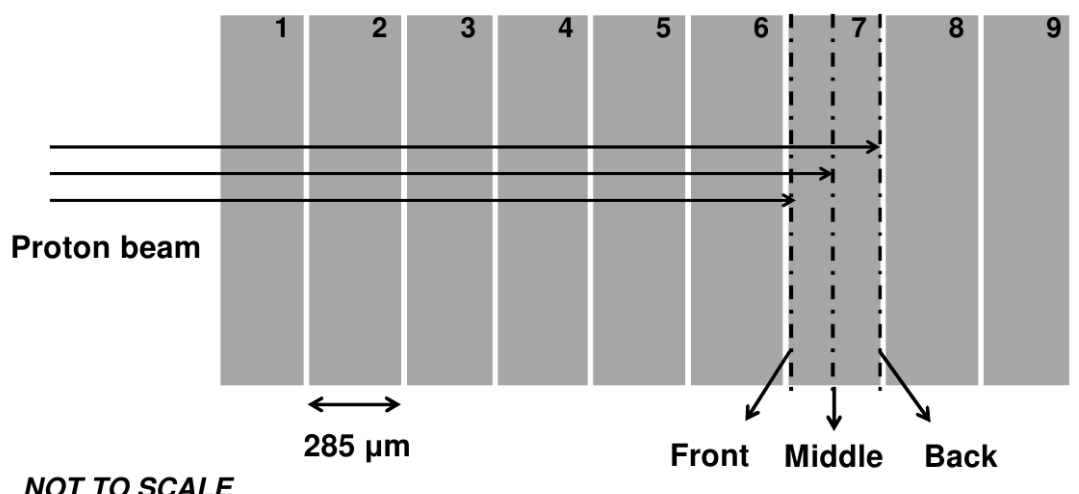

Figure 6.14: Schematic view of the stacked target that was irradiated with focused protons from the PET cyclotron. It shows three dot-dashed lines (front, middle, and back) used to assess proton beam energy at the entrance of the stacked target. These lines correspond to beam energy of 13.15, 13.75, and $14.3 \mathrm{MeV}$, respectively.

\subsubsection{Explaining on target beam inhomogeneity}

The experimental setup of the stacked target experiment was simulated by means of Geant 4 for a proton beam incident at $0^{\circ}$ and $2^{\circ}$, respectively. The stacked target was considered at a distance of $5 \mathrm{~cm}$ after a 20 -mm-diameter aluminum collimator at the end of the 2.4-m-long aluminum pipe (figure 6.15). Figure 6.16 shows the corresponding results obtained at the sixth EBT2 film of the two stacked targets that were irradiated with focused (beam incident at $0^{\circ}$ ) and defocused (beam incident at $2^{\circ}$ ) beams, respectively. 


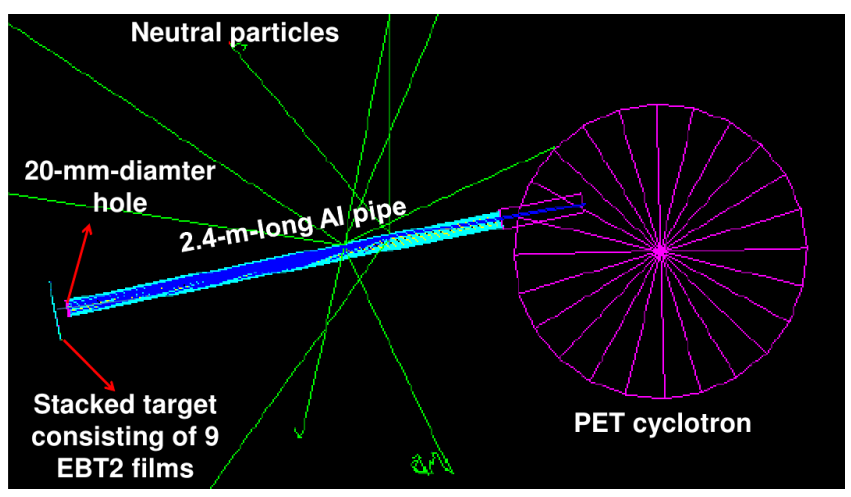

Figure 6.15: A view of the experimental setup of the stacked target experiment simulated by means of Geant 4 for a proton beam (dark blue) incident at $2^{\circ}$. The length of the aluminum pipe (cyan tube) is $2.4 \mathrm{~m}$, whereas the diameter of the circular hole at its end is $20 \mathrm{~mm}$. Nine EBT2 films, each with $285-\mu \mathrm{m}$ thickness, were positioned in-air and placed after the hole and a Havar ${ }^{\circledR}$ window that separates the irradiated stacked target from the vacuum in the pipe. Green trajectories correspond to neutral particles (neutrons or $\gamma$-rays). The violet circle represents the iron yoke of the cyclotron.
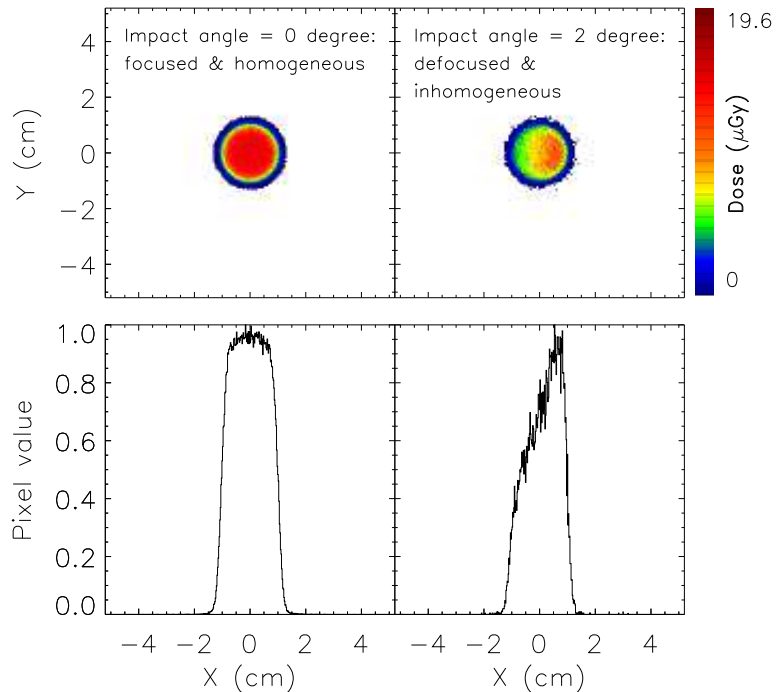

Figure 6.16: Simulation results obtained with the sixth EBT2 film of the two stacked target that were irradiated with focused (left) and defocused (right) beams. Despite the very low simulated statistics $(\sim 20 \mu \mathrm{Gy})$, it can be seen that the beam spot on the EBT2 film that was irradiated with focused beam is uniform, whereas the beam spot of the one that was irradiated with defocused beam is asymmetric. The corresponding central beam profiles for the two beam spots are shown at the bottom. 
These simulations were performed in order to investigate the asymmetry of beam spots on the irradiated EBT2 films with defocused protons (figures 6.9 and 6.10). Figure 6.16 shows that the dose is homogeneous in the beam region that was induced with focused beam with a steep profile. This result is consistent with the experimental one, where the beam spots on the EBT2 films that were induced by the focused protons are uniform (figures 6.9 and 6.10, focused beam). In addition to that, figure 6.16 shows that the irradiated area of the EBT2 film with defocused protons is asymmetric, where the right side of the beam spot has higher dose than the left one. This result is also consistent with the experimental one (figures 6.9 and 6.10, defocused beam). Therefore, the simulation results seem to indicate that the asymmetric beam spots that were induced by the defocused protons have their origin due to proton scattering inside the inner surface of the long aluminum pipe. In summary, beam homogeneity is repeatedly achieved by scanning the magnet current with the target shutter closed and the dose rate measurement system on, as shown in figure 6.8.

\subsubsection{Current-based dose estimation using published film- response data}

The irradiation setup which is shown in figure 6.3 has been used to irradiate 9 EBT2 films with focused protons from the PET cyclotron with different values of dose (figure 6.17). The films were irradiated one at a time during nine different irradiation.

The thin aluminum foil (figure 6.3) was connected to a modified in-house made electronics, where the total gain of the in-house developed electronics (figure 6.4) was decreased by a factor of 10 in order to accommodate measurements with larger ion source currents. Therefore, the time needed for target irradiation with a preset dose would be decreased.

In order to assess the absolute dose in the irradiated EBT2 films (figure 6.17), a published experimental data was used [Wang et al., 2012]. Eight EBT2 films irradiated with photons with 0,100, 200, 300, 400, 500, 600, and $700 \mathrm{cGy}$, respectively, were considered. These films were irradiated for providing a calibration dose curve. Reinhardt et al. [2012] concluded that the dosimetric response of EBT2 films to photon and proton beams is the same. No dependence on radiation type has been found between photon and proton exposure for the investigated EBT2 films, except for protons in the vicinity of the Bragg peak, where the dosimetric response of EBT2 films is below that expected (i.e., EBT2 films suffer 


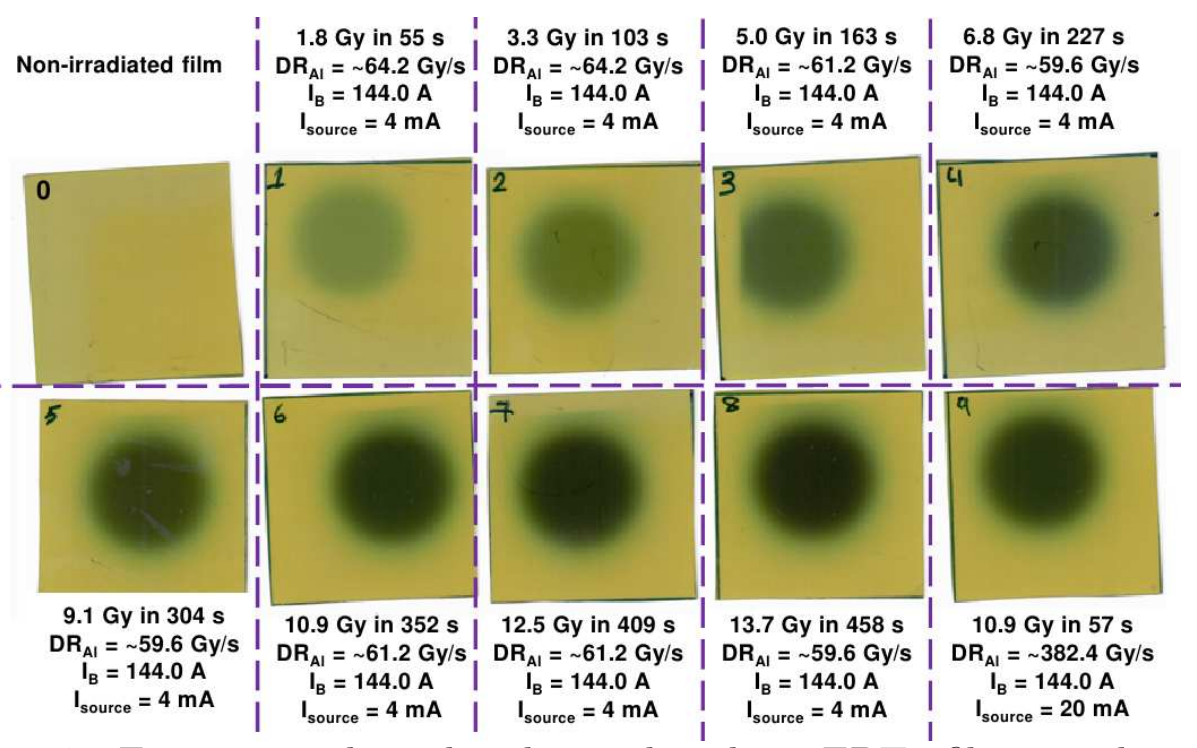

Figure 6.17: Experimental results obtained with 10 EBT2 films irradiated with protons at different values of dose using the developed irradiation setup. The films were positioned after the rotating slit perpendicular to the proton beam axis. The first film is a non-irradiated film. The seventh and tenth films were irradiated with the same value of dose, but at two different dose rates. It can be seen that the EBT2 film becomes darker with increasing absorbed dose.

from under-response to proton beams in the vicinity of the Bragg peak only).

Figure 6.18 shows the relation between the dose (X-ray dose) and film darkening (relative pixel color) obtained from the work of Wang et al. [2012]. The relative pixel color of a certain film is defined as the mean of the pixel color values for the film before irradiation minus the mean of the pixel color values in the uniform region for the same film after irradiation with a certain dose. A dose calibration equation (Wang's equation) was obtained from the fitting of the $\mathrm{X}$-ray dose versus relative pixel color (figure 6.18).

The X-ray dose in the irradiated EBT2 films with protons from the PET cyclotron (figure 6.17) was assessed using the dose calibration equation obtained in figure 6.18, as shown in figure 6.19. This figure shows that two independent methods for measuring dose (i.e., current-based dose and X-ray dose) are consistent within around 8\% accuracy. The current-based dose was measured using the developed irradiation system (figure 6.3). The values of proton dose (i.e., current-based dose) based on equation 6.5, which are the ones computed with the dosimetry system developed for ICNAS, where multiplied by $75 \%$ after taken into consideration the ratio of the measured beam current with respect to the 
dose in a circular region with radius $R_{1}$ containing the central uniform region of a beam spot 1 .

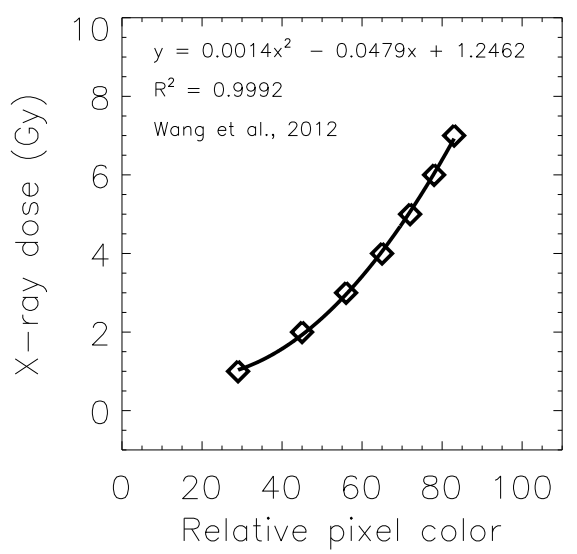

Figure 6.18: Absolute dose (X-ray dose) in the irradiated EBT2 calibration films Wang et al., 2012] as a function of relative pixel color (film darkening). It can be seen that a parabolic fit to the data allows for a high $R^{2}$ (coefficient of determination) value to be obtained.
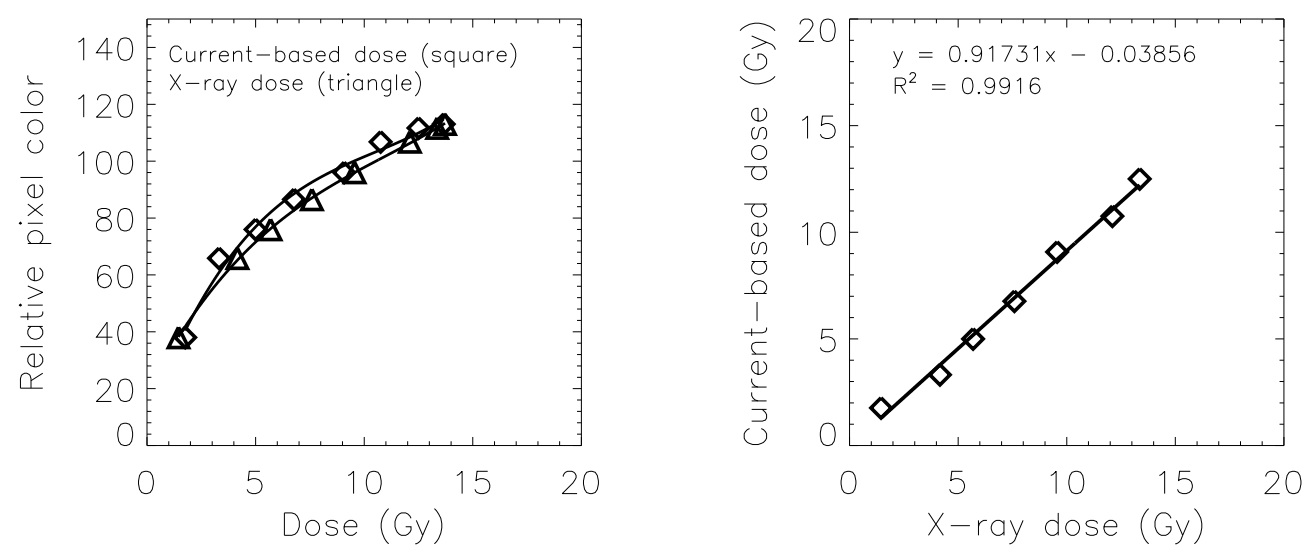

Figure 6.19: Left: Relative pixel color for the irradiated EBT2 films with protons as a function of dose (i.e., current-based dose (squares) and X-ray dose (triangles). Right: Current-based dose as a function of X-ray dose. The linearity between both values can be seen, being consistent within around $8 \%$ accuracy.

\footnotetext{
${ }^{1}$ See section 6.3 .3 .3
} 


\subsubsection{Assessing target dose by means of absolute 2D dosime- try with Gafchromic ${ }^{\circledR}$ EBT2 film}

In order to improve the accuracy $(8 \%)$ of the assessed absolute dose in the irradiated EBT2 films, which was evaluated using a published experimental data [Wang et al., 2012], an experimental setup using Gafchromic ${ }^{(\mathrm{R})}$ EBT2 films was used. For that, the irradiation setup which is shown in figure 6.3 was used to irradiate EBT2 films with focused protons from the PET cyclotron with different values of dose. In order to assess the absolute dose in the irradiated films, the 2D dosimetry system of the Department of Radiotherapy of Coimbra University Hospital Center was used.

\subsubsection{X-ray film dosimetry}

In order to validate the developed proton irradiation setup at ICNAS, the film dosimetry system of the Department of Radiotherapy of Coimbra University Hospital Center has been used. Twenty EBT2 films have been irradiated with $4 \mathrm{MV}$ photon beam (Clinac 600C - Varian Medical Systems, Palo Alto, CA). These films were irradiated with different values of dose ranging from $0.5 \mathrm{~Gy}$ up to $13.0 \mathrm{~Gy}$ for providing a calibration dose curve. Additionally, another 17 EBT2 films were irradiated with protons from the PET cyclotron using the developed irradiation setup at ICNAS.

Before irradiation, all EBT2 films to be used for calibration purposes were scanned. For that, an inexpensive flatbed scanner, Epson Perfection V700 (Seiko Epson Corporation, Nagano, Japan) was used in transmission mode to scan the films. The images were acquired with films positioned with the same orientation and placed at a specific position on the scanner bed with the use of a frame, ensuring a reproducible positioning (figure 6.20). All EBT2 films were marked in order to keep the original portrait orientation information. The films were scanned surrounded by a film template to keep the same scanning conditions for all films.

The region of interest (ROI) of $16 \times 16$ pixels size was defined at the center of each film and the mean pixel value $P V$ was evaluated. The use of a control film $(C F)$ is needed to correct any drift effects. Insertion of a $3 \times 3 \mathrm{~cm}^{2}$ unexposed EBT1 film piece at one corner of the scanner bed (figure 6.20), was used as the control film. The film piece is subjected to the same scanning conditions and therefore the output scanner signal of the film piece can be used to partially 


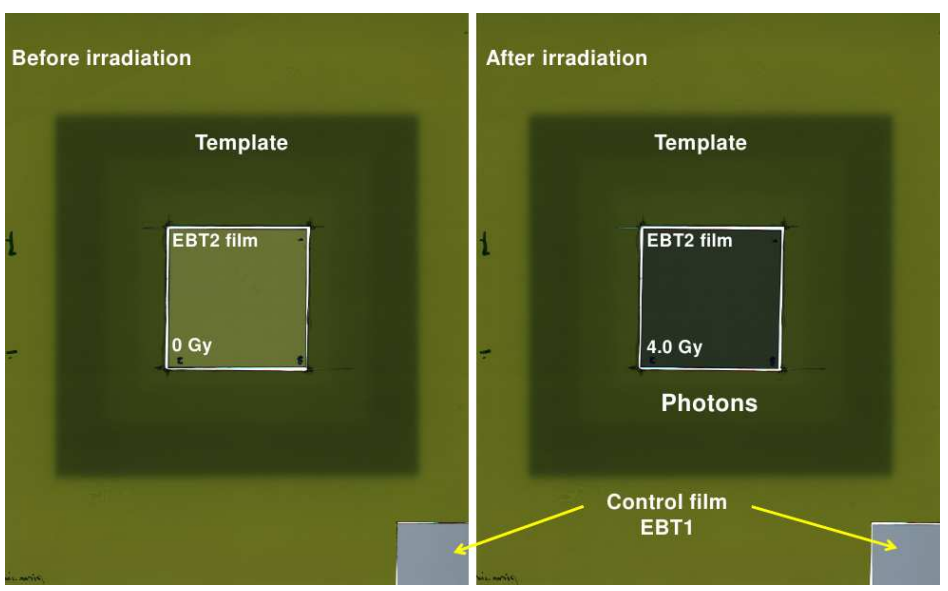

Figure 6.20: Experimental results obtained with an EBT2 film scanned before (left) and after (right) irradiation with a $4 \mathrm{MV}$ photon beam from a clinical linear accelerator. The control EBT1 film and the template around the scanned EBT2 films can be seen.

correct system fluctuations. The mean corrected pixel value $P V_{\text {corr }}$ is calculated using the following equation:

$$
P V_{\text {corr }}=P V \cdot \frac{P V_{c f}^{b k g}}{P V_{c f}},
$$

where $P V_{c f}^{b k g}$ and $P V_{c f}$ are the $C F$ mean pixel value without and with insertion of an EBT2 film at the scanner flatbed, respectively.

During the scanning process of an EBT2 film, the intensity of the transmitted light is reported as the image pixel value $(P V)$ and it is converted to optical density $O D$ using the following formula:

$$
O D=\log _{10} \frac{P V^{b k g}}{P V},
$$

where $P V^{b k g}$ is the mean pixel value from a background scanned image (empty scanner).

The calibration EBT2 films were irradiated in sequence with a $4 \mathrm{MV}$ photon beam from a clinical linear accelerator with different values of dose ranging from $0.5 \mathrm{~Gy}$ up to $13.0 \mathrm{~Gy}$. The actual dose values were measured at the center of each film using a PTW (Physikalisch-Technische Werkstätten) thimble $0.125 \mathrm{~cm}^{3}$ ionization chamber. The films were scanned once again after one week of irra- 
diation to prevent uncertainties caused by post irradiation growth (figure 6.20, right). Images from pre-irradiation and post-irradiation sessions were analyzed using an in-house developed SciLab code. For all images, the red component was extracted and the red net optical densities net $O D_{r}$ were calculated using the following expression:

$$
\text { net } O D_{r}=O D_{r}^{e}-O D_{r}^{u}
$$

where $O D_{r}^{e}$ and $O D_{r}^{u}$ are the measured optical densities for post-irradiation (exposed) and pre-irradiation (unexposed), respectively. Finally, a sensitometric calibration curve was established using the dose steps in the calibration films (figure 6.21, left). The calibration curve function $f_{c a l}$ was obtained from the fitting of measured dose $D$ versus mean net $O D_{r}$ values.

$$
f_{c a l}(x)=\frac{x-a}{b x^{2}+c x+d}
$$

with $x=$ net $O D_{r}$ and the four parameters a, b, c, and $\mathrm{d}$ being found to be $-3.6056 \times 10^{-7}, 0.10778497,-0.26127781$ and 0.19341574 , respectively.

In order to validate the stability of the 2D dosimetry system, another 8 EBT2 films were irradiated with a $4 \mathrm{MV}$ photon beam from the clinical linear accelerator with different values of dose. Three of them were irradiated on the same day of irradiation of the 20 EBT2 films that were used to establish the calibration dose curve (figure 6.21, left). The remaining five films were irradiated one week after, on the same day where the 17 EBT2 films were irradiated with protons from the PET cyclotron using the developed irradiation setup at ICNAS. The absolute dose in these 8 EBT2 films were assessed using the 2D dosimetry system with a precision better than 1\% (figure6.21, right). In this figure, two points show three and four different irradiation in one week different time.

\subsubsection{Validating proton dose based on X-ray film dosimetry}

Seventeen EBT2 films were irradiated with protons from the PET cyclotron using the irradiation setup developed at ICNAS. Figure 6.22, right, shows one example of a film irradiated with $3.8 \mathrm{~Gy}$. One week after that irradiation, the absolute dose in the irradiated films were evaluated using the $2 \mathrm{D}$ film dosimetry of the Department of Radiotherapy of Coimbra University Hospital Center. 

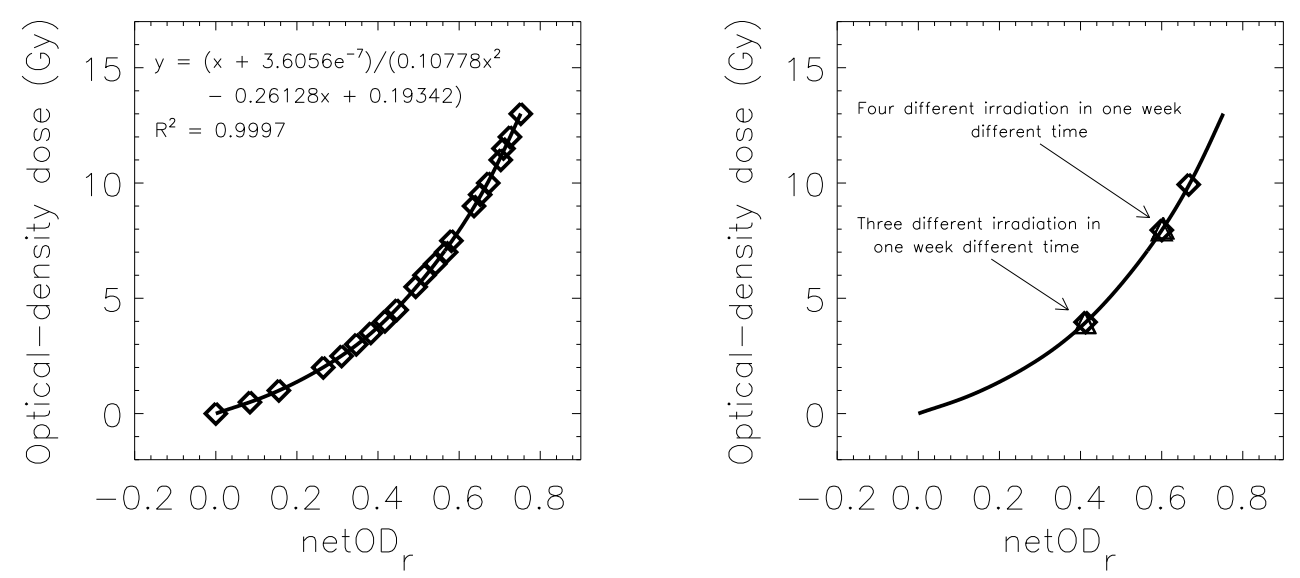

Figure 6.21: Left: Absolute dose (optical-density dose) in the irradiated EBT2 calibration films as a function of netOD $D_{r}$. It can be seen that the relation between both values is parabolic. Right: Validation of the 2D dosimetry system by irradiation 8 EBT2 films with $4 \mathrm{MV}$ photon in one week different time. The optical-density dose in these films were assessed with uncertainty less than $1 \%$.

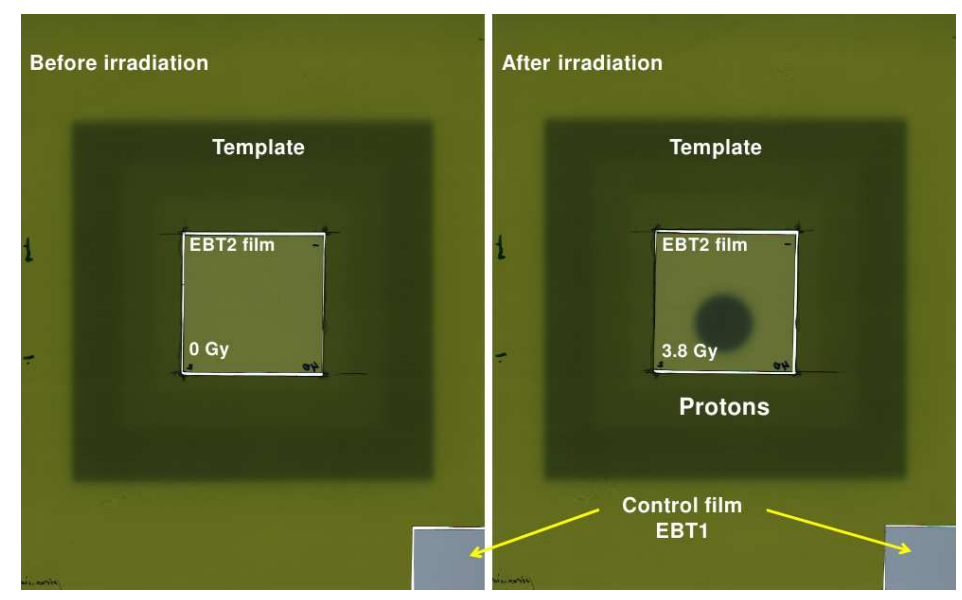

Figure 6.22: Experimental results obtained with an EBT2 film scanned before (left) and after (right) irradiation with protons using the developed irradiation setup at ICNAS. The control EBT1 film and the template around the scanned EBT2 films can be seen.

\subsubsection{Estimating out-of-field dose}

In order to calculate the ratio of the measured beam current in respect to the dose in a circular region with radius $R_{1}$ containing the central uniform region of a beam spot, the pixel color values of the beam spot were assessed (figure 6.23). For that, the netOD $D_{r}$ (equation 6.8) and the corresponding optical-density dose 
(equation 6.9) were taken into account. This allows to calculate the opticaldensity dose within the uniform circular region $\left(R_{1}=9 \mathrm{~mm}\right)$ and in the circularringed regions around the uniform area (figure 6.23, left).

The pixel-color original beam profile and the corresponding dose-based beam profile were plotted on the same figure, as shown in figure 6.23, right. The ratio of the measured beam current in a selected region within the uniform part of the beam spot can be assessed using equation 6.10,

$$
\text { Current ratio }\left[R_{1}\right]=\frac{\pi\left(R_{1}^{2}-R_{0}^{2}\right) D_{1}}{\sum_{i=1}^{N} \pi\left(R_{i}^{2}-R_{i-1}^{2}\right) D i}
$$

where $D_{i}$ is the optical-density dose in the corresponding region $\left(D_{1}\right.$ is the opticaldensity dose in the uniform region), $R_{i}$ is the radius of the corresponding circular area on the beam spot (figure 6.23, left). For the uniform circular region (figure 6.23), $R_{0}$ and $R_{1}$ equal 0 and $9 \mathrm{~mm}$, respectively. Based on that, the ratio of the measured beam current in the uniform circular area (18-mm diameter) in respect to the whole beam current measured was found to be $55 \%$.
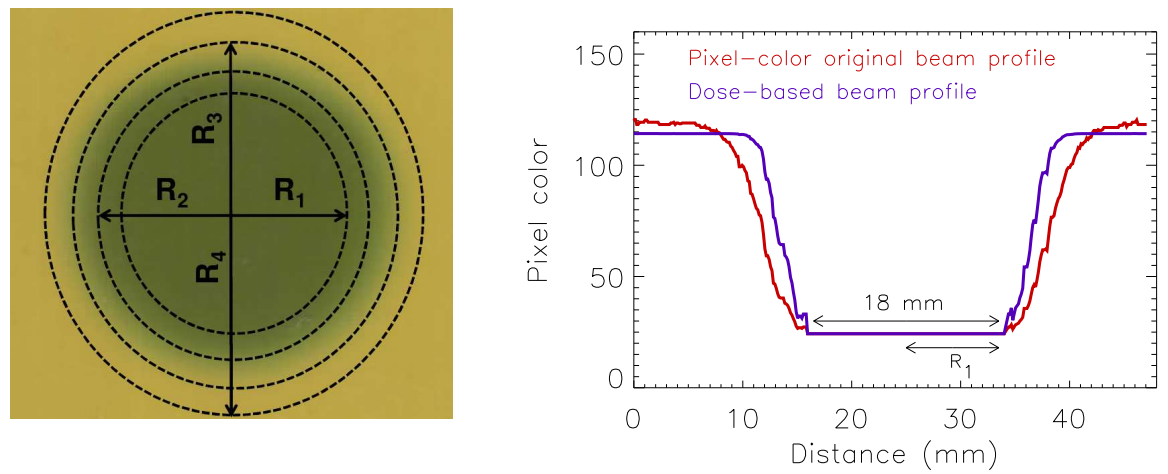

Figure 6.23: Left: A scan copy of an irradiated EBT2 film with focused protons at ICNAS. The dose in the uniform circular area $\left(R_{1}\right)$ and in circular-ringed areas around the uniform one were calculated based on equation 6.9. Right: The brown profile corresponds to the pixel-color original beam profile of the beam spot, whereas the violet profile corresponds to a corrected profile based on dose measurements (dose-based beam profile). The diameter of the uniform region $(18 \mathrm{~mm})$ on the beam spot is considered to be the distance between two points on the beam profile, where the pixel color between these two points oscillates within $\pm 2 \%$.

We observe an excess of the measured dose signal in respect to the film opticaldensity dose of the order of $20 \%$. This may be explained by the fact that protons 
Chapter 6. PET cyclotron based irradiation setup for proton radiobiology and radiophysiology

suffer from multiple scattering inside the beam pipe. This effect decreases beam energy to the extent of creating low-energy protons capable of producing a secondary current on the aluminum foil, yet not escaping either this film nor the aluminum sheet covering it. Figure 6.24 shows this fact. It was obtained by means of Geant 4 simulations and it allows to see that $20 \%$ of the initial protons scattered in the Havar foils reach the measuring aluminum foil with an energy inferior to $2.5 \mathrm{MeV}$. At this energy and below the protons lack enough energy to leave the foil, therefore contributing to the measured current (i.e., "increased" measured dose) but without inducing a contribution to an effective target dose (i.e., film darkening). For this reason, the values of proton dose based on equation 6.5, which are the ones computed with the dosimetry system developed for ICNAS, were multiplied by $75 \%$ (55\% from equation 6.10, and $20 \%$ from the results explained in figure 6.24).
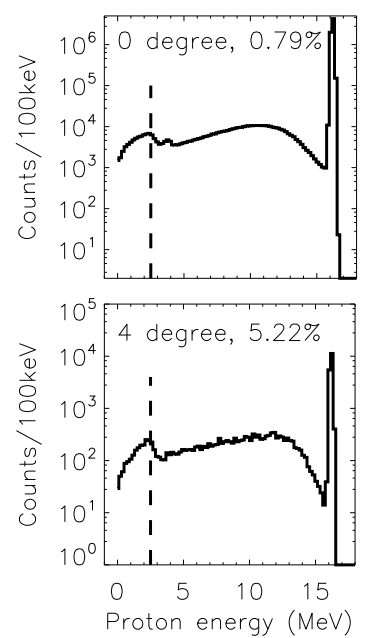
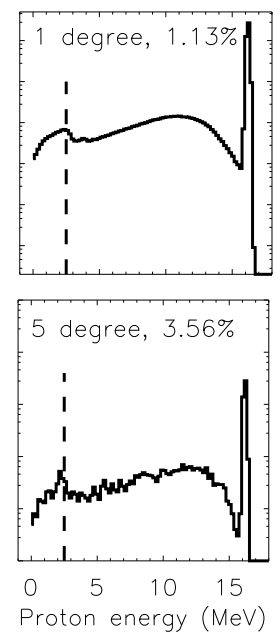
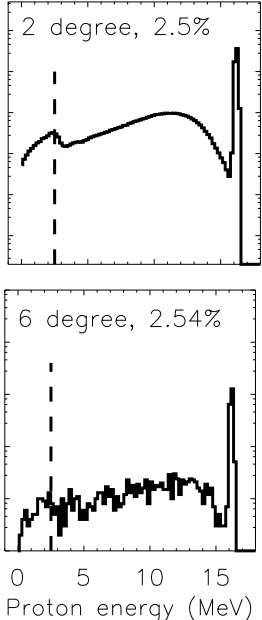
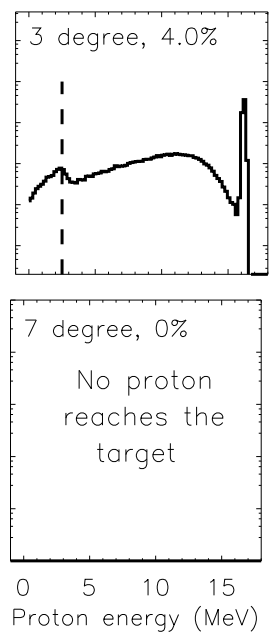

Figure 6.24: Geant4 simulation results obtained for proton spectrum at the end of the long pipe of the irradiation setup, for proton beam incident at angles of $0^{\circ}$, $1^{\circ}, 2^{\circ}, 3^{\circ}, 4^{\circ}, 5^{\circ}, 6^{\circ}$, and $7^{\circ}$, respectively. The ratio of the number of protons with energies inferior to $2.5 \mathrm{MeV}$ to the total number of protons, which were scored by a perfect detector, are shown on the corresponding figures. The simulation results are presented for $10^{9}$ initial protons. Twenty percent of all protons do not escape the aluminum foil.

Simulation results shown in figure 6.25 reveal that by deviating the initial proton beam direction just by $1^{\circ}$ from its orthogonal direction to a target (air), result in the range of the proton beam in air decreasing from few meters down to a few centimeters. This result reinforces that part of the initial protons suffer from multiple scattering inside the long aluminum pipe. Thus these protons lose part of their energy in the beam path before reaching the target positioned at the end of the long pipe. 

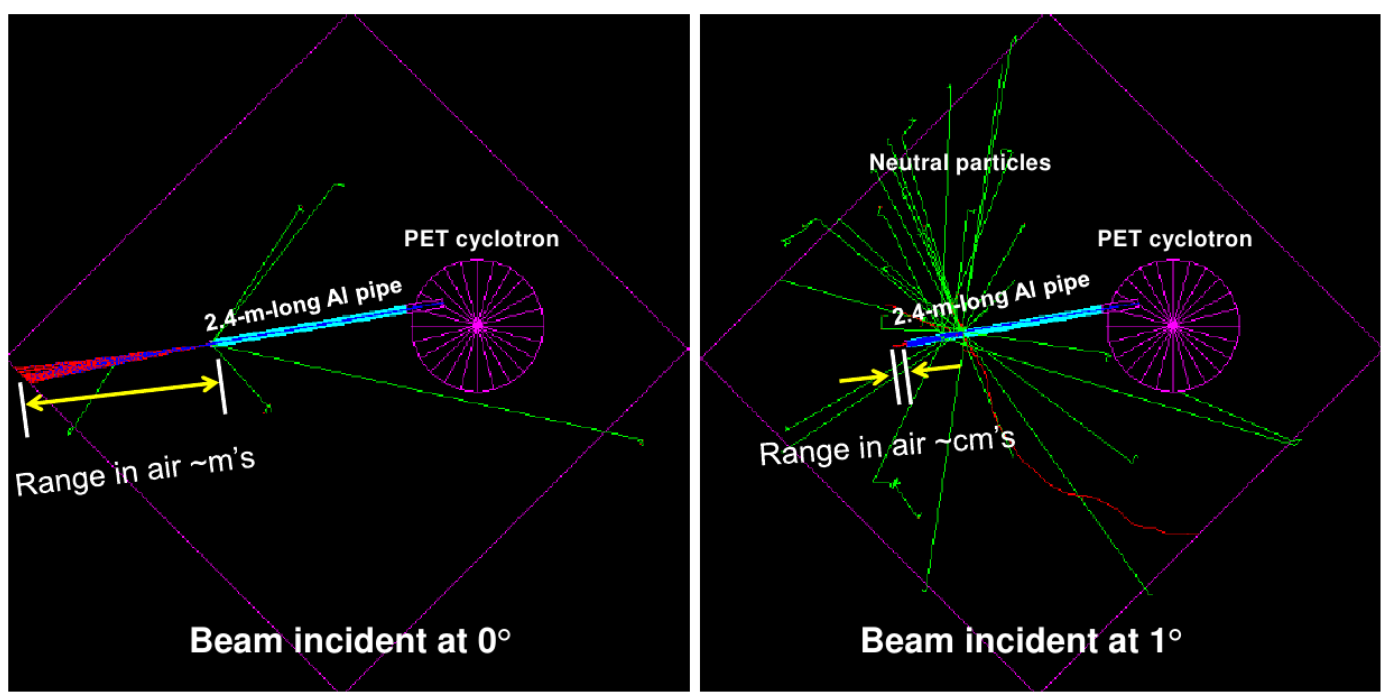

Figure 6.25: Two top views of the irradiation setup simulated by means of Geant4. The length of the aluminum pipe (cyan tube) is $2.4 \mathrm{~m}$, whereas the diameter of the circular hole at the end of the aluminum pipe is $20 \mathrm{~mm}$. The left view is presented for a 100 initial protons (dark blue) for an initial proton direction orthogonal to the 20 -mm-diameter hole, whereas the right one is presented for $1 \mathrm{k}$ initial protons for an initial proton beam direction at an angle of $1^{\circ}$ from its orthogonal direction to the hole at the end of the long pipe. Green trajectories correspond to neutral particles (neutrons or $\gamma$-rays), whereas red trajectories correspond to negative particles (electrons arising from ionization in air). The violet circle represents the iron yoke of the cyclotron, whereas the violet square represents the concrete walls of the bunker.

\subsubsection{Proton current-based dose versus optical-density dose}

Figure 6.26 shows the relation between the current-based dose, which was measured using the developed irradiation setup at ICNAS, and the optical-density dose, which was assessed using the 2D film dosimetry system of the Department of Radiotherapy of Coimbra University Hospital Center. This figure shows that the two independent methods for measuring dose are consistent within less than $2 \%$ accuracy, due to the oscillations in the measured pixel values. The figure also shows one point that represent four different irradiation at three hour different time and different dose rates. 


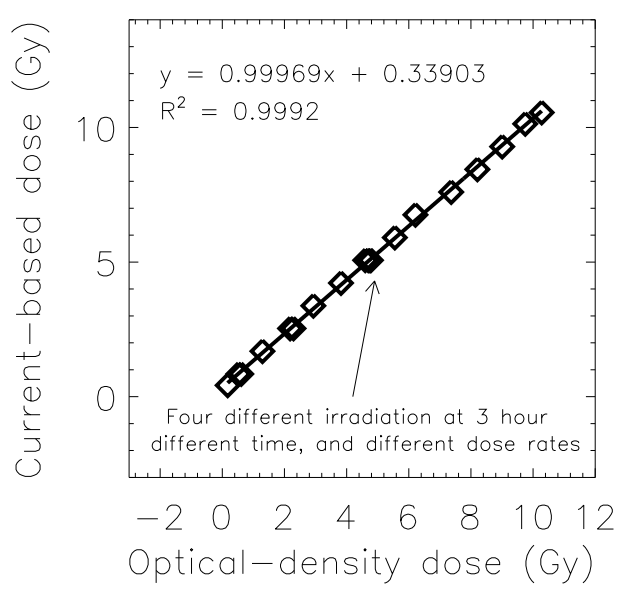

Figure 6.26: Current-based dose as a function of optical-density dose. The linearity between both values can be seen. In addition, the repeatability in the measured current-based dose was also evaluated.

\subsection{A 2D position multi-well setup for proton radiobiology}

By using the developed dosimetry system at ICNAS, a homogeneous beam spot on target with a diameter of $18 \mathrm{~mm}$ can be controlled. This allows controlled irradiation of cell cultures located in typical biological multi-cavity dishes with diameters of $16 \mathrm{~mm}$ e.g., 24-cavity cell culture plate 1. In order to irradiate several cell cultures, the 2D multi-well radiobiological setup shown in figure 6.27 was constructed.

The bottom of a cell culture plate has a uniform thickness, allowing for distortion-free observation. The plate has a non-reversible cover with a condensation ring to minimize evaporation and the risk of air contamination. Cell culture plates are made of opticallv clear and non-pvrogenic polvstvrene [Ted Pella Inc., team, 2014]. Each of the 24 cavities of the cell culture plate will be irradiated with protons, one at a time. The plate will be fixed on an aluminum support (figure 6.27), which will be positioned in air next to the rotating disk perpendicularly to the proton beam axis and in alignment with the 23-mm-diameter hole at the end of the 2.4-m-long aluminum pipe of the developed irradiation setup.

It is planned that the process of alignment of the cavities of a multi-cavity cell

\footnotetext{
${ }^{1}$ Cell culture-treated plates are designed for a wide range of applications including general cell growth experiments, cloning studies, and in vitro testing.
} 


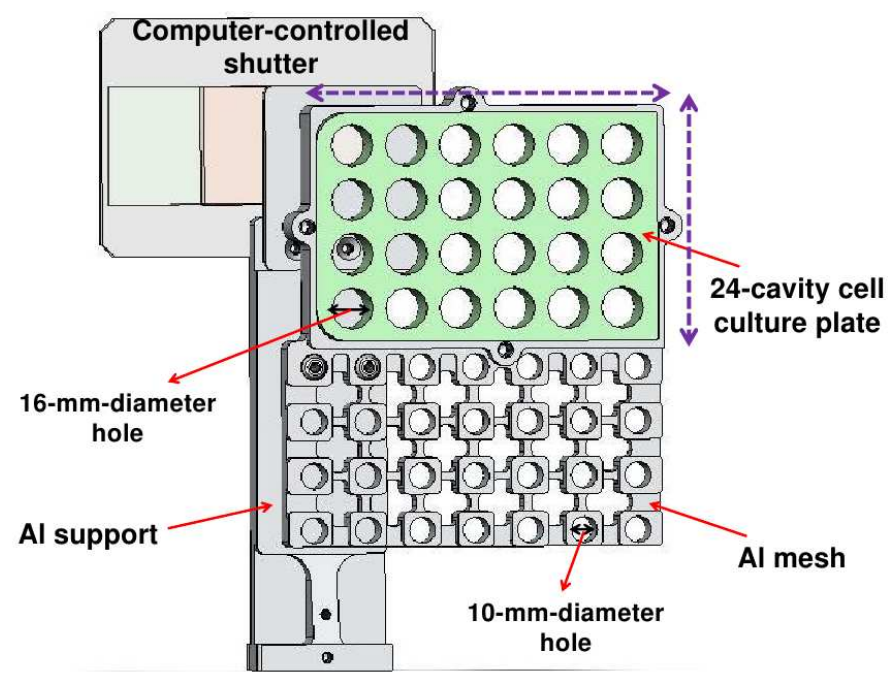

Figure 6.27: Schematic diagram of a 24-cavity cell culture plate control system. It shows a computer-controlled shutter, a 24-cavity cell culture plate, an aluminum mesh, and an aluminum support. The aluminum mesh is composed of $2810-\mathrm{mm}-$ diameter holes, which are used in order to align the 24 cavities of the cell culture plate positioned in the beam path, one at a time.

culture plate with the beam path will be done, one by one, with a $2 \mathrm{D}$ automatically controlled system similar to figure 6.27. Therefore, the irradiation of several cell cultures located in the biological multi-cavity dish will be fully controlled from outside the bunker of the cyclotron.

\subsection{Summary and outlook}

A precise dosimetry system with accelerated proton beams from a PET cyclotron was developed and validated for the first time using the 2D film dosimetry system of the Department of Radiotherapy of Coimbra University Hospital Center. A transmission thin $(20-\mu \mathrm{m}$-thick) aluminum foil is readout by in-house made transimpedance electronics, providing online dose information via a $\mathrm{C} / \mathrm{C}++$ program. The beam monitor is able to perform automatic irradiation and quantification of doses of several Gy down to one hundred mGy, with a precision better than $2 \%$. This was achieved by installing a 2.4-m-long aluminum pipe allowing for decreasing proton flux by three orders of magnitude. In addition, a rotating disk with a slit of $0.5 \mathrm{~mm}$ width located at a disk radius of $150-\mathrm{mm}$ was installed at the end of the long pipe. This rotating slit allows for decreasing target dose rates by 
a factor of $5 \times 10^{-4}$. The beam uniformity is repeatedly achieved by scanning the magnet current with the target shutter closed and the dose rate measurement system on. This scanning process is needed to determine the magnet current that produces focused protons, hence producing a homogeneous target dose. The dose on target can be varied between 18-mm diameter down to 0.5 -mm diameter. Hence, the system is now prepared to perform radiobiological and radiophysiological experiments and other proton-based dosimetric studies. 


\section{Chapter 7}

\section{Conclusions and outlook}

A cyclotron facility has been recently installed at ICNAS for production of radioisotopes for medical use. The accelerator is an IBA model Cyclone ${ }^{\circledR}$ 18/9 -HC with fixed energy of $18 \mathrm{MeV}$ for protons. Such facility is equipped with eight exit ports suitable for scientific research.

A fast and precise technique for measuring position, range, and energy of the proton beam from a PET cyclotron is provided. By using a plastic scintillator and a D-link Ethernet-based camera, the proton beam range from the PET cyclotron at ICNAS was measured with a precision of $0.1 \mathrm{~mm}$. The same technique was utilized to measure the Bragg peak of the proton beam. Additionally, another method was used to measure the Bragg peak of the proton beam. This was achieved by using a stacked target consisting of several aluminum foils interleaved with polyethylene sheets. The current of secondary electrons liberated (secondary current) from the aluminum foils were measured using in-house made transimpedance electronics. The measured Bragg peaks are consistent with simulations performed using the SRIM/TRIM simulation toolkit. The initial energy of the proton beam from the PET cyclotron at ICNAS was assessed to be $18 \mathrm{MeV}$.

A precise dosimetry system using an accelerated proton beam coming from the PET cyclotron at ICNAS was successfully developed, characterized, calibrated, and validated for the first time using the 2D film dosimetry system of the Department of Radiotherapy of Coimbra University Hospital Center. This beam monitor is needed for successful radiobiology, radiophysiology, and other dosimetric studies with ICNAS proton beams. The monitoring system was adjusted to the special characteristics of the proton beam from the PET cyclotron. The system is intended to be applicable to quasi-static proton beams and able to 
measure total dose as well as dose rate every second, which makes it suitable for real-time target dosimetry. A $20-\mu$ m-thick aluminum transmission foil is readout by in-house made transimpedance electronics, providing online dose information via a $\mathrm{C} / \mathrm{C}++$ program. This detector is based on measuring secondary electrons liberated when the proton beam traverses thought the thin aluminum foil. The monitoring system is able to perform automatic irradiation and quantification of doses of several Gy down to one hundred mGy, with a precision better than $2 \%$. This was achieved by fitting the PET-dedicated cyclotron with a 2.4-mlong beam transport line, as a natural extension of one of the cyclotron beam ports, while keeping available the other seven beam ports for PET radionuclides production. The long aluminum pipe allows for decreasing proton flux by three orders of magnitude. Next to the 2.4-m-long aluminum pipe, a 150-mm-radius rotating disk with a slit of $0.5 \mathrm{~mm}$ width was positioned. This rotating slit decreases target dose rates by a factor of $5 \times 10^{4}$. The beam uniformity is achieved by scanning the magnet current with the target shutter closed and the dose rate measurement system on. This scanning process is needed to determine the magnet current that produces focused protons, hence producing a homogeneous target dose. Therefore, the irradiation setup is now prepared to perform radiobiological and radiophysiological experiments and other proton-based dosimetric studies.

Objectives for near future work include performing radiobiological, radiophysiological, and dosimetric studies at the developed irradiation setup, therefore fulfilling its first design goals. Non-exhaustive examples include the study of cell culture and in-vivo tissue responses to concomitant irradiation with protons and natural or fabricated biochemical agents such as vitamin C, other antioxidants, and chemotherapeutical agents. In the radiation protection scientific field, radiation response models for very-low dose regimes should also be investigated: hormesis, linear-no-threshold, linear-threshold, and linear quadratic, among others.

By using the developed dosimetry system at ICNAS, a homogeneous beam spot on target with a diameter of $18 \mathrm{~mm}$ can be controlled, this allows controlled irradiation of cell cultures located in typical biological multi-cavity dishes with diameters of $16 \mathrm{~mm}$ e.g., 24-cavity cell culture plate. In order to irradiate several cell cultures, an automatic 2D positioning system for multi-well cell cultures irradiation is being constucted. 
Appendices 



\section{Appendix A}

\section{Havar ${ }^{\circledR}$ window}

In the late 1940's the Hamilton watch company developed for the first time the Havar ${ }^{\circledR}$ alloy as an unbreakable mainspring material. As watches moved from spring to battery power, the thin Havar ${ }^{\circledR}$ strips found a second life in high strength, non-magnetic, and corrosion resistant pressure sensing diaphragms for process control equipment [Pinto, 2010; Robinson, 2003].

$\operatorname{Havar}^{\circledR}$ alloy, which is a heat treatable cobalt base alloy that provides very high strength, is used in many applications such as pressure diaphragms, power springs. gap spacers in magnetic heads. and target foils in nuclear phvsics [HPM team, 2013]. Havar ${ }^{\mathrm{R})}$ is characterized by a relatively high density $\left(8.3 \mathrm{~g} / \mathrm{cm}^{3}\right)$ and high thermal conductivity $\left(13.0 \mathrm{Wm}^{-1} \mathrm{~K}^{-1}\right)$. Its physical properties are listed in table A.1. On the other hand, its composition is reported in table A.2.

Table A.1: Physical properties of Havar ${ }^{\circledR}$ alloy [HPM team, 2013].

\begin{tabular}{ll}
\hline Property & Value \\
\hline Density & $8.304 \mathrm{~g} / \mathrm{cm}^{3}$ \\
Melting point & $1480^{\circ} \mathrm{C}$ \\
Electrical resistivity @ R. T. & $92.0 \mu \Omega \mathrm{cm}$ \\
Thermal expansion coefficient $\left(1\right.$ to $\left.50^{\circ} \mathrm{C}\right)$ & $12.5 \times 10^{-6} \mathrm{C}^{-1}$ \\
Thermal conductivity & $13.0\left[\mathrm{Wm}^{-1} \mathrm{~K}^{-1}\right]$ \\
Magnetic attraction & None \\
\hline
\end{tabular}


Table A.2: Nominal composition of Havar ${ }^{\circledR}$ alloy [HPM team, 2013].

\begin{tabular}{ll}
\hline Element & Elemental percentage (\%) \\
\hline Cobalt & 42.0 \\
Chromium & 19.5 \\
Nickel & 12.7 \\
Tungsten & 2.7 \\
Molybdenum & 2.2 \\
Manganese & 1.6 \\
Carbon & 0.2 \\
Iron & 19.1 \\
\hline
\end{tabular}




\section{Appendix B}

\section{Geant4 simulation toolkit}

\section{B.1 Introduction}

Geant4 (geometry and tracking 4) Agostinelli et al., 2003; Allison et al., 2006] is a public, costless Monte-Carlo (MC) based software toolkit developed at CERN 1 . It simulates the passage of particles through matter. It is a very flexible solution with innumerous models of physics processes in a wide range of energies. It has been initially created for high energy applications up to about $100 \mathrm{TeV}$ and now allows for very low energy applications down to a few eV. Geant4 simulates all kind of particles. It can cover hadronic, electromagnetic, and optical interactions. It has libraries to a large set of materials, elements, and long-lived particles. A large number of processes, models, cross-sections, and simulation parameters are available and they have to be carefully selected depending on the application. It is designed to be used as object-oriented language and it is implemented in $\mathrm{C}++$. Its areas of application include high energy, nuclear and accelerator physics, as well as studies in medical and space science.

The hadronic interactions with Geant4 are divided into three major categories: (a) cascade state models; (b) pre-equilibrium state models; and (c) equilibrium state models. The cascade state describes the intra-nuclear projectile-nucleon

\footnotetext{
${ }^{1}$ http://www.geant4.org/geant4/
} 
and nucleon-nucleon interactions after the projectile enters the nucleus. The two main models for this phase are the Bertini cascade and the binary cascade. The Bertini model is a classical cascade. It generates the final state for hadron inelastic scattering by simulating the intra-nuclear cascade. In this model incident hadrons collide with protons and neutrons in the target nucleus and produce secondaries which in turn collide with other nucleons. The Binary model is a modeling sequence similar to Bertini, except that, hadron-nucleon collisions are handled by forming resonances, which then decay according to their quantum numbers.

The main code for modelization of the pre-equilibrium state is the precompound model, which takes a nucleus from a highly-excited set of particle-hole states down to equilibrium energy by emitting protons, neutrons, deuterons, tritium nuclei, helium-3 nuclei, and alphas. After the pre-equilibrium phase, the nucleus is supposed to be left in an equilibrium state, in which the excitation energy is shared by a large number of nucleons. The equilibrium state is also known as nuclear evaporation or nucleus de-excitation phase. At the end of the thermalization process, the nucleus is defined by its mass, charge, and excitation energy. If the excitation energy exceeds the separation energy 1 , further particles can be emitted. The equilibrium models of Geant 4 describe the emission of photons, nucleons, and light fragments from this residual state Geant4 team, 2013; Lara and Wellisch, 2000].

The Geant4 simulation toolkit was utilized, after validation with published experimental data with protons of similar energy Verbinski and Burrus, 1969], in order to explain experimental results described in this thesis. Furthermore, Geant4-based Monte Carlo was utilized in order to evaluate the possibility of using one beamline of ICNAS PET cyclotron for an out-of-yoke irradiation setup.

\section{B.2 Monte-Carlo validation}

A proton beam with $18-\mathrm{MeV}$ energy was shot in vacuum through a $25.2-\mu \mathrm{m}$-thick ${ }^{9} \mathrm{Be}$ target in order to reproduce the experimental setup (figure B.1) utilized by Verbinski and Burrus [Verbinski and Burrus, 1969] to measure double differential cross sections for thin-target neutron production. The resulting neutron emission polar angle $\theta$ was scored by means of a perfect detector implemented within

\footnotetext{
${ }^{1}$ Separation energy is the energy required to separate particles from a molecule, atom, or nucleus.
} 
Geant4. Code and physics libraries previously optimized [Pinto, 2010] were utilized in this work, namely Geant4 version 9.3.p01, with the QGSP_BERT_HP physics package activated. Figure B.2 shows a comparison of experimental results with those obtained with Geant4. A remarkably good agreement between simulation and experimental data can be verified.

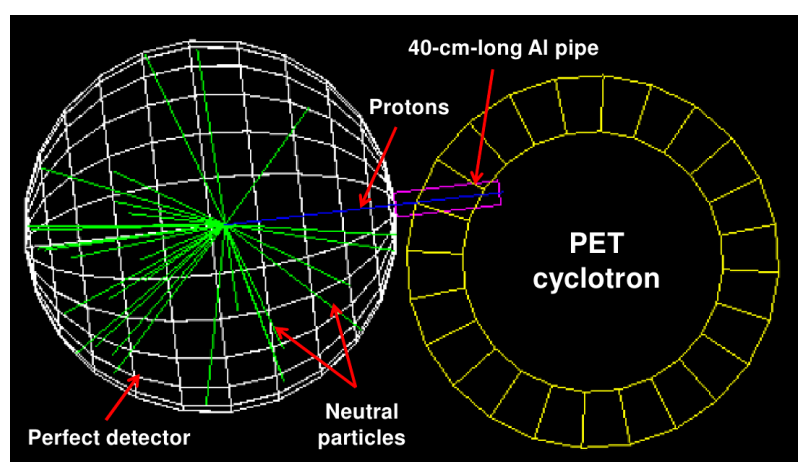

Figure B.1: View of the experimental setup of Verbinski and Burrus experiment. A $25.2-\mu$ m-thick ${ }^{9}$ Be target (not shown) is irradiated with $18 \mathrm{MeV}$ proton beam (blue) from a PET cyclotron (yellow). The resulting neutron emission (green) polar angle $\theta$ was scored by means of a perfect sphere detector (white).

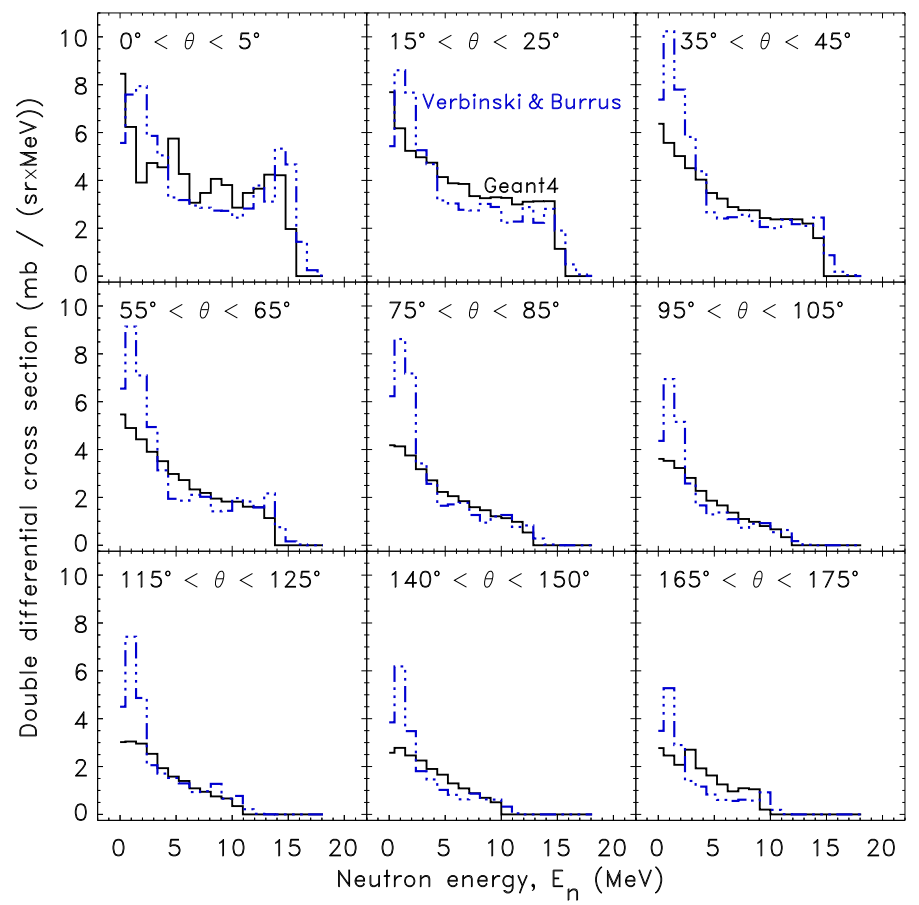

Figure B.2: Double differential cross sections $\left(\mathrm{d}^{2} \sigma /(\mathrm{dEd} \theta)\right)$ comparing experimental data from Verbinski and Burrus [Verbinski and Burrus, 1969] and results obtained with Geant4. 
Appendix B. Geant4 simulation toolkit 


\section{Appendix C}

\section{Frequency-to-voltage converter}

An in-house developed electronics has been assembled at ICNAS in order to drive a proper current to the motor of the computer-controlled shutter from a PicoScope 2203 waveform generator via a frequency-to-voltage converter. Figure C.1 shows the basic connection diagram for frequency-to-voltage conversion. This circuit is based on the LM331 amplifier, which converts the input frequency into a proportional voltage that is extremely linear to the input frequency. The input-output relation is given by equation C.1 [CT team, 2013; TI team, 2013].

$$
\operatorname{Vout}[V]=[R 4 /(R 5+R 6)] \times R 1 C 1 \times 2.09 \times \text { Fin }
$$

A PicoScope 2203 is used to generate a square wave of $2 \mathrm{~V}$ amplitude ( $4 \mathrm{~V}$ peak-to-peak amplitude). The calculated output voltage values, based on equation C.1, are 0.96 and $1.21 \mathrm{~V}$ for input frequency values of 4 and $5 \mathrm{kHz}$, respectively. The output signals from the frequency-to-voltage converter circuit are shown in figure C.2, left, for both values of frequency.

In order to filter out the oscillations with 4 and $5 \mathrm{kHz}$ from the output signals of the frequency-to-voltage converter, a low pass filter was added directly after the frequency-to-voltage converter circuit. After that, a voltage offset adjustment circuit followed by a voltage amplifier with gain of 2 were added right after the low pass filter (figure C.3). These pieces were used in order to get a negative voltage, 


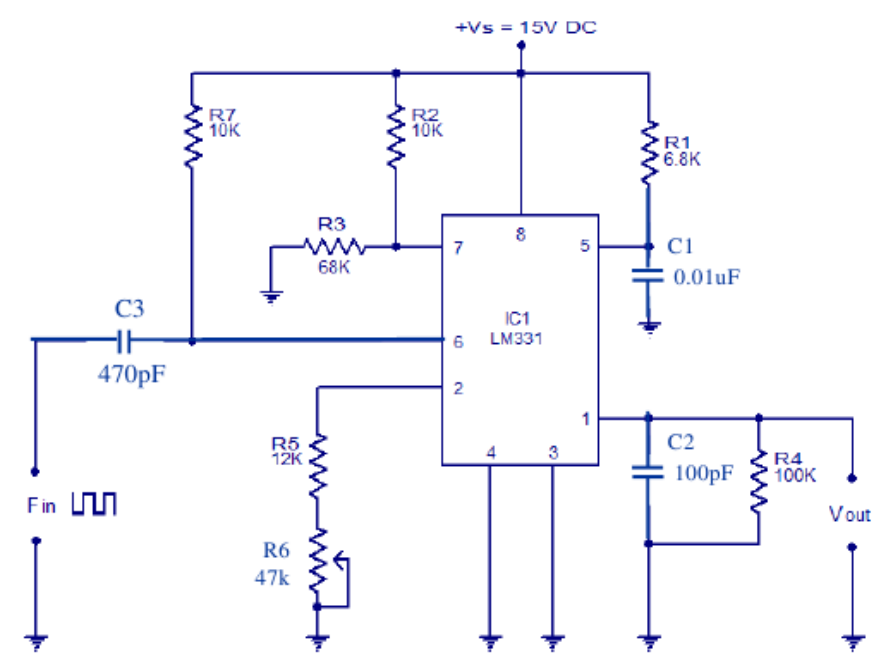

Figure C.1: Schematic diagram of the frequency-to-voltage converter using the LM331 amplifier. This converter was used for driving a suitable current to the motor of the shutter before, during, and after target irradiation. In addition to the LM331 amplifier, several resistors and capacitors were used. As a result, the output voltage (Vout), which is available across the load resistor $R 4$, is proportional to the input frequency (Fin).
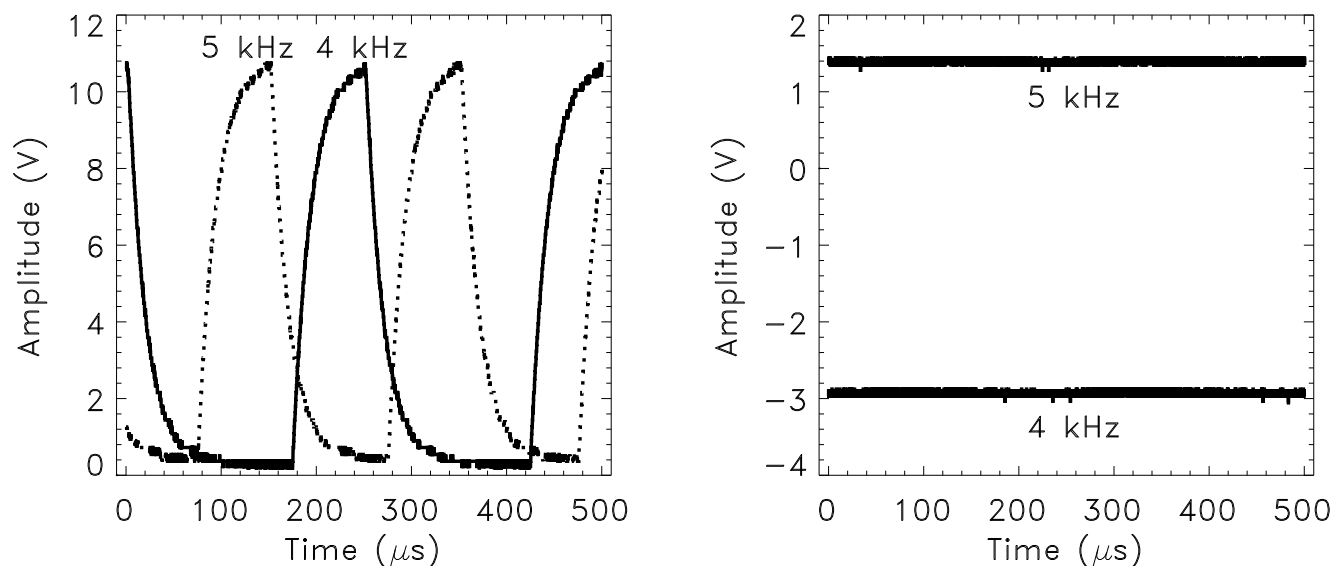

Figure C.2: Signals from the frequency-to-voltage converter (figure C.1) for an input waveform (square wave) with $4 \mathrm{~V}$ peak-to-peak amplitude (left figure). The dotted signal corresponds a frequency of $5 \mathrm{kHz}$, whereas the solid one corresponds to a frequency of $4 \mathrm{kHz}$. The right figure shows the corresponding signals after passing through a low pass filter, a voltage offset adjustment, and a voltage amplifier with gain of 2 . 
in addition to the positive one. Since the frequency-to-voltage converter, which is shown in figure C.1 provides just a positive voltage. Both voltages are necessary for the operation of the shutter in the proper way. Therefore, by adding those electronic pieces, the output signal can be adjusted, where positive and negative voltages can be generated by adapting the frequency of the generated square wave from the PicoScope 2203 waveform generator. For instance, the average voltage values of the output signals are 1.39 and $-2.94 \mathrm{~V}$ for input frequency values of 5 and $4 \mathrm{kHz}$, respectively (figure C.2, right).

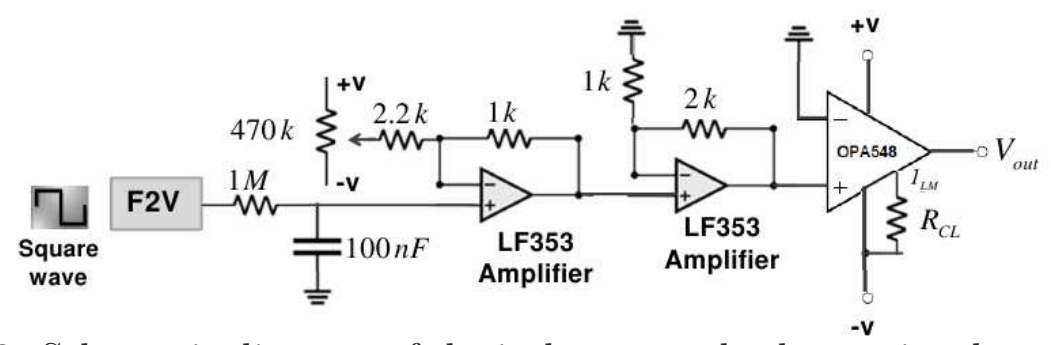

Figure C.3: Schematic diagram of the in-house made electronics that was used directly after the frequency-to-voltage converter. It shows a low pass filter, a voltage offset adjustment, a voltage amplifier, and a high-current amplifier (OPA548).

Since the driven current from the frequency-to-voltage converter to the shutter motor is not enough for the motor to operate, therefore a high-voltage highcurrent operational amplifier (OPA548) was used, as shown in figure C.3.

In summary, the shutter can be controlled by choosing the suitable frequency value for the generated square signals from the PicoScope 2203 waveform generator. Several tests were performed at ICNAS cyclotron after assembling the inhouse developed electronics of the shutter and the in-house made transimpedance amplifiers of the transmission thin aluminum foil that provides beam current information. Experimental results reveal that the in-house developed electronics systems for beam readout and control of beam exposure have a common ground, where the shutter electronics was introducing some noise to the output signals from the aluminum foil. This issue was solved by using a CAMAC system and a laptop with isolated grounds instead of the frequency-to-voltage convertor for beam exposure control. Consequently, the electronics of the computer-controlled shutter becomes totally separated from that of the thin aluminum foil. Thus, the shutter electronics does not disturb the output signals from the aluminum foil. 
Appendix C. Frequency-to-voltage converter 


\section{References}

Advanced Science Research (2008). Biological effects of clustered DNA damage - mutation induction by DNA lesions in close proximity. JAEA R\&D review, page 71.15

Agostinelli, S., Allison, J., Amako, K., Apostolakis, J., Araujo, H., et al. (2003). Geant4 - a Simulation Toolkit. Nucl. Instrum. Meth., A 506:250 - 303. 66, 145

Ahmed, S. (2007). Physics and Engineering of Radiation Detection. Academic Press is an imprint of Elsevier. 11, 28, 31, 32, 33, 34, 35, 38, 39, 40, 43, 50, 52, 53, 63, 64,

AIST team (2009). Establishment of the mammography dose standard - Towards achieving safe and secure breast cancer examination. Translation of AIST press release of March 5, 2009. Available at http://www.aist.go.jp/aist_e/latest_research/2009/20090413/20090413.html. 53, 54

Allison, J., Amako, K., Apostolakis, J., Araujo, H., Dubois, P. A., et al. (2006). Geant4 Developments and Applications. IEEE Trans Nucl. Sci., 53(1):270 278. 66, 145,

Amaldi, U. and Kraft, G. (2005). Radiotherapy with beams of carbon ions. Rep. Prog. Phys., 68:1861 - 1882. 21

Archambault, L., Poenisch, F., Sahoo, N., Robertson, D., Lee, A., et al. (2012). Verification of proton range, position, and intensity in IMPT with a 3D liquid scintillator detector system. Med. Phys., 39(3):1239 - 1246. 78 
Bal, C., Bravin, E., Lefèvre, T., Scrivens, R., and Taborelli, M. (2005). Scintillating screens study for LEIR/LHC heavy ion beams. Proceedings DIPAC 2005, POM013 - Lyon, France, pages 57 - 59. 29

Barkas, W., Birnbaum, W., and Smith, F. (1956). Mass-ratio method applied to the measurement of L-meson masses and the energy balance in pion decay. Phys. Rev., 101(2):778 - 795. 10,

Birks, J. (1951). The efficiency of organic scintillators. Proc. Phys. Soc., A 64:847 - 870. 32

Blakely, E. and Chang, P. (2004). Late effects from hadron therapy. Radiother. Oncol., 73(2):S134 - S140. 18

Borca, V., Pasquino, M., Russo, G., Grosso, P., Cante, D., et al. (2013). Dosimetric characterization and use of Gafchromic ebt3 film for IMRT dose verification. J. Appl. Clin. Med. Phys., 14(2):158 - 171. 36

Borovsky, J., McComas, D., and Barraclough, B. (1988). The secondary-electron yield measured for 5-24 protons on aluminum-oxide and gold targets. Nucl. Instrum. Meth., B 30(2):191 - 195. 92, 94

Breidenbach, M., Frisch, J., Markiewicz, T., and Smith, S. (2004). Diamond detectors. LC B-I Meeting, SLAC, 2 June. 41, 43

Brown, A. and Suit, H. (2004). The centenary of the discovery of the Bragg peak. Radiother. Oncol., 73(3):265 - 268. 17, 18

Brown, G., Halback, K., Harris, J., and Winick, H. (1983). Wiggler and undulator magnets - A review. Nucl. Instrum. Meth., 208:65 - 77. xxii

Butson, M., Yu, P., Cheung, T., and Metcalfe, P. (2003). Radiochromic film for medical radiation dosimetry. Materials Science and Engineering, R 41:61 120. 35

Cancer Research UK (2012). World cancer factsheet - world cancer burden (2008). This report was prepared by the section of cancer information at international agency for research on cancer (IARC), with support from the statistical information team, Cancer Research UK. 1, 4

Chu, W., Ludewigt, B., and Renner, T. (1993). Instrumentation for treatment of cancer using proton and lightion beams. Rev. Sci. Instrum., 64(8):2055-2122. 20 
Crespo, P. (2005). Optimization of in-beam positron emission tomography for monitoring heavy ion tumor therapy. PhD thesis, Technical University of Darmstadt. 8, 10, 11, 13, 14, 19, 89,

CT team (2013). Frequency to voltage converter using LM331. CT - Circuits Today. Available at http://www.circuitstoday.com/frequency-to-voltageconverter-using-lm331. Online, June. 149

Cunha, M. (2010). Radiobiology with proton cyclotron beams: A viability study. Master's thesis, University of Coimbra. 19, 58, 61

Debus, J., Scholz, M., Haberer, T., Peschke, P., Jäkel, O., et al. (2003). Radiation tolerance of the rat spinal cord after single and split doses of photons and carbon ions. Radiat. Res., 160(5):536 - 542. xix

Denham, J. and Hauer-Jensen, M. (2002). The radiotherapeutic injury - a complex 'wound'. Radiother. Oncol., 63(2):129 - 145. xix

Dosanjh, M., Jones, B., and Meyer, R. (2010). ENLIGHT and other EU-funded projects in hadron therapy. Br. J. Radiol., 83(994):811 - 813. 17

Drisko, J., Chapman, J., and Hunter, V. (2003). The use of antioxidant therapies during chemotherapy. Gynecol. Oncol., 88(3):434 - 439. xix

Durante, M. and Loeffler, J. (2010). Charged particles in radiation oncology. Nat. Rev. Clin. Oncol., 7(1):37 - 43. 3, 8

EBT2 team (2009). Gafchromic EBT2 self-developing films for radiotherapy dosimetry. 1361 Alps Road Wayne NJ 07470 973- 628-3831/-3531. Revision 1. 35, 36, 37,

Eljen Technology team (2013). Plastic scintillators. Available at http://www.eljentechnology.com/index.php/products/plastic-scintillators.

Online, June. 32

Elsässer, T. and Scholz, M. (2007). Cluster effects within the local effect model. Radiat. Res., 167(3):319 - 329. 15

Elsässer, T., W.K.-Weyrather, Friedrich, T., Durante, M., Iancu, G., et al. (2010). Quantification of the relative biologial effectiveness for ion beam radiotherapy: Direct experimental comparison of proton and carbon ion beams and a novel approach for treatment planning. Int. J. Radiation Oncology Biol. Phys., 78(4):1177 - 1183. Xix 
ENLIGHT (2013). Science. The European network for light-ion hadron therapy - a multidisciplinary platform aimed at a coordinated effort towards ion beam research in Europe. Available at http://enlight.web.cern.ch/science. 4

European Nuclear Society (2013). Dose. European Nuclear Society - largest nuclear society for science and industry. Available at http://www.euronuclear.org/info/encyclopedia/d/dose.htm. Online, December. 63, 64,

Feinendegen, L. (2005). Evidence for beneficial low level radiation effects and radiation hormesis. $B J$ Radiol, 78(925):3 - 7. xix

Ferlay, J., Shin, H., Bray, F., Forman, D., Mathers, C., et al. (2010). Estimates of worldwide burden of cancer in 2008: GLOBOCAN 2008. Int. J. Cancer, 127(12):2893 - 2917. 1

Ferlay, J., Soerjomataram, I., Ervik, M., Dikshit, R., Eser, S., et al. (2014). Globocan 2012 - Estimated cancer incidence, mortality and prevalence worldwide in 2012. Available at http://globocan.iarc.fr/Default.aspx. (c) Copyright - international agency for research on cancer (IARC). 1, 2

Fokas, E., Kraft, G., An, H., and Engenhart-Cabillic, R. (2009). Ion beam radiobiology and cancer: Time to update ourselves. Biochim. Biophys. Acta., 1796(2):216 - 229. 8

Forck, P. (2011). Lecture notes on beam instrumentation and diagnostics. Lecture notes, JUAS (Joint University Accelerator School), Archamps, France. 23, 25, 26, 27, 29, 30, 31, 40, 43, 44, 46, 47, 48, 49, 50, 51, 53, 105, 115,

Forshier, S. (2002). Essentials of radiation biology and protection. Delmar, New York, first edition. 16

Freeman, T. (2008). Site focus: UFPTI. The University of Florida proton therapy institute (UFPTI) in Jacksonville. Available at http://medicalphysicsweb.org/cws/article/research/34366. 20.

Fukushima, Y., Hamada, M., Nishio, T., and Maruyama, K. (2006). Development of an easy-to-handle range measurement tool using a plastic scintillator for proton beam therapy. Phys. Med. Biol., 51(22):5927 - 5936. 78

Gajewski, J. (2013). 2D thermoluminescence dosimetry for scanning ion beam. Symposium on applied nuclear physics and innovative technologies, Kraków, Poland, 4th June 2013. Available at http://www.ips.if.uj.edu.pl/. 34 
Geant4 team (2013). Physics reference manual. Version: Geant4 10.0. 6 December. 146

Gei, O. (2002). Thermoluminescence detectors. Available at http://nucleonicsonline.com/657.html. 33

Goitein, M. (2007). Radiation oncology: a physicist's-eye view. Biological and medical physics, biomedical engineering, Springer-Verlag New York, LLC. 20]

Gottschalk, B. (2004). Passive beam spreading in proton radiation therapy. Technical report, Harvard high energy physics laboratory. Available at http://huhepl.harvard.edu/ gottschalk. 13

Gottschalk, B., Koehler, A., Schneider, R., Sisterson, J., and Wagner, M. (1993). Multiple Coulomb scattering of $160 \mathrm{MeV}$ protons. Nuclear Instruments and Methods in Physics Research Section B: Beam Interactions with Materials and Atoms, 74(4):467 - 490. 13

GSI Nachrichten (1999). Proposal for a dedicated ion beam facility for cancer therapy. Feature, 1:16 - 19. 3

Haberer, T. (2002). Advances in charged particles therapy. Nuclear Physics in $21^{\text {st }}$ Century: Int. Nucl. Phys. Conf. INPC 2001. AIP Conf. Proc., Vol. 610, pages 157 - 166. Available at https://getinfo.de/app/Advances-in-Charged-ParticleTherapy/id/BLCP\%3ACN043691090. 3

Haberer, T., Becher, W., Schardt, D., and Kraft, G. (1993). Magnetic scanning system for heavy ion therapy. Nucl. Instrum. Meth., A 330:296 - 305. 22

Hada, M. and Georgakilas, A. (2008). Formation of clustered DNA damage after high-LET irradiation: a review. J. Radiat. Res., 49(3):203 - 210. 16

Hall, E. and Giaccia, A. (2006). Radiobiology for the radiologist. Lippincott William and Wilson. 12

Hamamatsu Photonics K. K. (2006). Photomultiplier tubes - Basics and applications. Hamamatsu Photonics K. K. - Electron Tube Devision, third edition. 29

Highland, V. (1975). Some practical remarks on multiple scattering. Nucl. Instrum. Meth., 129(2):497 - 499. 14

HPM team (2013). Technical data sheet - Havar ${ }^{\circledR}$. HPM - Hamilton Precision Metals. Available at http://www.hpmetals.com/spec-sheets/index.aspx, June. 143, 144 
Hsu, P., Weng, P., Lin, S., and Du, D. (1998). The response of the thermoluminescent dosimeter CaF2:Tm to protons in the energy range 15-30 MeV. Appl. Radiat. Isot., 49(12):1619 - 1624. 34

IAEA and ICRU (2008). Relative biological effectiveness in ion beam therapy. Technical report series, ISSN 00741914; no. 461, jointly sponsored by the international atomic energy agency (IAEA) and the international commission on radiation units and measurements (ICRU). 1, 3, 4, 16, 19,

IBA team (2013). Cyclone ${ }^{\circledR}$ 18/9 - moving ahead, delivering more. Cyclone ${ }^{\circledR}$ 18/9 - IBA RadioPharma Solutions. Online, March 2013. Available at http://www.iba-radiopharmasolutions.com/. 57, 58

ICRU (1970). Linear energy transfer. ICRU report no. 16, Washington. 11, 12

ImageJ team (2004). Software package ImageJ. Image processing and analysis in Java. Available at http://rsbweb.nih.gov/ij/. 68, 79, 121, 122

Iriso, U. and Pérez, F. (2006). Synchrotron radiation monitors at ALBA. Proceedings of EPAC 2006, THPLS056 - Edinburgh, Scotland, pages 3410 - 3412. 25

ISP team (2010). Gafchromic EBT2 film for a contemporary RT environment. (C) 2010 International Specialty Products. Designed \& Printed in USA. Available at http://gafchromic.com/. 36, 37

Jackson, S. (2002). Sensing and repairing DNA double-strand breaks. Carcinogenesis, 23(5):687 - 696. 15

Janni, J. (1982). Energy loss, range, path length, time-of-flight, straggling, multiple scattering, and nuclear interaction probability: in two parts. Part 1. For 63 compounds. Part 2. For elements $1 \leqslant z \leqslant 92$. Atomic Data and Nuclear Data Tables, 27(4 - 5):341 - 529. 9, 13

Janovsky, I. and Ross, C. (1993). The IRS thermoluminescent dosimetry system. Report. Ionizing radiation standards institute for national measurement, standards national research council, Ottawa, Ontario, K1A 0R6 - PIRS-0369. 33

Jones, D. (2006). The w-value in air for proton therapy beams. Radiation Physics and Chemistry, 75:541 - 550. 53

Jung, M., Rothard, H., Gervais, B., and Grandin, J. (1996). Transport of electrons induced by highly charged $\mathrm{Ni} 74 \mathrm{MeV} / \mathrm{u}$ and $\mathrm{Cu} 9.6 \mathrm{MeV} / \mathrm{u}$ ions in carbon: A 
study of target-thickness-dependent electron yields. Phys. Rev., A 54(5):3632 - 3636. 93

Jung, R., Ferioli, G., and Hutchins, S. (2003). Single pass optical profile monitoring. Proceedings DIPAC 2003, IT03 - Mainz, Germany, pages 10 - 14. 30. 31

Karzmark, C. (1964). Secondary emission monitor as a linear accelerator electron beam dose monitor. Review of Scientific Instruments, 35(12):1646 - 1652. 46

Kim, H., Park, S., Kim, Y., Ha, J., Kang, S., et al. (2006). A parallel plate ionization chamber for a proton beam monitoring. Journal of the Korean Physical Society, 48:213 - 217. 59, 91

Kim, K., Cho, Y., Hong, I., Park, B., Yun, S., et al. (2007). Proton beam energy measurement using semiconductor detectors at the $45 \mathrm{MeV}$ test beamline of PEFP. Proceedings of PAC0\%, FRPMN055 - Albuquerque, New Mexico, USA, pages 4126 - 4128. 59

Kim, K., Jung, M., Ra, S., and Lee, S. (2010). Development of an external Faraday cup for beam current measurements. Journal of the Korean Physical Society, 56(6):2104 - 2107. 26, 27, 91

Kirby, D. (2011). Radiation dosimetry of conventional and laser driven particle beams. PhD thesis, University of Birmingham. [35, 36

Kirby, D., Green, S., Palmans, H., Hugtenburg, R., Wojnecki, C., et al. (2010). LET dependence of GafChromic films and an ion chamber in low-energy proton dosimetry. Phys. Med. Biol., 55(2):417 - 433. 36, 37

Knoll, G. (2000). Radiation detection and measurement. Wily. 28, 31, 33, 38, 40, 60, 63

Koschar, P., Kroneberger, K., Clouvas, A., Burkhard, M., Meckbach, W., et al. (1989). Secondary-electron yield as a probe of preequilibrium stopping power of heavy ions colliding with solids. Phys. Rev., A 40(7):3632 - 3636. 92, 93

Koziol, H. (2010). Beam diagnostics for accelerators. Lecture notes, CERN, Geneva, Swizterland. 23, 29, 46, 47, 48, 49

Kraft, G. (2000). Tumor therapy with heavy charged particles. Prog. Part. Nucl. Phys., 45:473 - 544. 8, 11, 14, 17, 19, 77, 89, 115, 
Kraft, G. (2003). Radiobiological effects of highly charged ions - their relevance for tumor therapy and radioprotection in space. F.J. Currell (ed.), the physics of highly and multiply charged ions - (c) 2003 Kluwer Academic Publisher. Vol. 1, chapter 5, pages $149-196$. 3

Kraft, G. (2007). Tumor therapy with heavy ions - physical and biological basis; technical realization at GSI; clinical results, volume 191 of Pitman Research Notes in Mathematics Series. Verein zur Frderung der Tumortherapie mit schweren Ionen e.V. www.gsi.de/informationen/verein-tuthe/, Darmstadt, Germany, ISBN: 3-9811298-2-2, 978-3-9811298-2-3. 17, 22

Lara, V. and Wellisch, J. (2000). Pre-equilibrium and equilibrium decays in Geant4. In Proc. 9th Int. Conf. Calorimetry in High Energy Physics, Annecy, France. 146

Leo, W. (1994). Techniques for nuclear and particle physics experiments, a how-to approach. Springer-Verlag. 9, 10, 28, 29, 31, 38, 50,

LHC team (2013). Measurement of beam current. Available at http://www.lhccloser.es/1/4/7/1. Online, November. 45

LTspice (2013). Ltspice - linear technology - simulation program with integrated circuit emphasis. Available at http://www.linear.com/designtools/software/. 100

Lutz, G. (1999). Semiconductor radiation detectors. Springer, ISBN 978-3-54071678-5 Springer Berlin Heidelberg New York. 38, 40

Martisíková, M. and Jäkel, O. (2010). Dosimetric properties of Gafchromic EBT films in monoenergetic medical ion beams. Phys. Med. Biol., 55:3741 - 3751. 36

Mothersill, C. and Seymour, C. (2004). Radiation-induced bystander effects and adaptive responses - the yin and yang of low dose radiobiology? Mutation Research, 568(1):121 - 128. 区x

Nakamura, K., Hagiwara, K., Hikasa, K., Murayama, H., Tanabashi, M., et al. (2010). Particle physics booklet, volume G 37. Journal of Physics, Particle Data Group, MS 50R6008, Lawrence Berkeley National Laboratory, Berkeley, CA 94720-8166, USA. 14

Nave, R. (2013). Threshold for relativistic effects. HyperPhysics. Avaliable at http://hyperphysics.phy-astr.gsu.edu/hbase/relativ/rellim.html. Online, October. 24 
Niederlaender, E. (2006). Causes of death in the EU. Statistics in Focus Population and Social Conditions, pages 1 - 11. ISSN: 1024-4352, Catalogue number: KS-NK-06-010-EN-N, (c) European Communities. 1

NSEP team (2011). Semiconductor detectors. NSEP - Nuclear Safeguards Education Portal. Available at http://nsspi.tamu.edu/nsep/courses/basic-radiationdetection/semiconductor-detectors/introduction/introduction. 40

Odier, P., Ludwig, M., and Thoulet, S. (2009). The DCCT for the LHC beam intensity measurement. Proceedings DIPAC 2009, MOPD45 - Basel, Switzerland, pages $143-145$. 46

Papash, A. and Alenitsky, Y. (2008). Commercial cyclotrons. Part I: commercial cyclotrons in the energy range $10-30 \mathrm{MeV}$ for isotope production. Physics of Particles and Nuclei, 39(4):597 - 631. 55, 57, 58, 59, 62, 77

Park, K., Lee, W., Jung, Y., Kim, D., Lee, H., et al. (2006). Development of beam profile monitor for cyclotron. Proceedings of EPAC 2006, TUPCH070 Edinburgh, Scotland, pages 1169 - 1171. 48, 49

Peeters, A., Grutters, J., Pijls-Johannesma, M., Reimoser, S., Ruysscher, D. D., et al. (2010). How costly is particle therapy? Cost analysis of external beam radiotherapy with carbon-ions, protons and photons. Radiother. Oncol., 95(1):45 $-53.18$

Pinto, M. (2010). Implementation of a cyclotron-based neutron beamline: A viability study. Master's thesis, University of Coimbra. 58, 59, 62, 143, 147,

Potiriadis, C., Clouvas, A., Rothard, H., Pauly, N., Dubus, A., et al. (2005). Influence of the target thickness on the backward and forward electron emission characteristics induced by protons incident on thin carbon foils. Nucl. Inst. Meth., B 230:466 - 470. 92

PTCOG (2013). Particle therapy facilities in operation (incl. patient statistics) in August 2013. PTCOG - Particle Therapy Co-Operative Group. Online, August 2013. Last update: August 27 th , 2010. Available at http://ptcog.web.psi.ch/ptcentres.html. 5, 6

Raich, U. (2005). Instrumentation in small low energy machines. Proceedings DIPAC 2005, ITMM01 - Lyon, France, pages 1 - 5. 26

Reinhardt, S., Hillbrand, M., Wilkens, J., and Assmann, W. (2012). Comparison of Gafchromic EBT2 and EBT3 films for clinical photon and proton beams. Med. Phys., 39(8):5257 - 5262. 123, 126 
Renner, T., Chu, W., Ludewigt, B., Nyman, M., and Stradtner, R. (1989). Multisegmented ionization chamber dosimetry system for light ion beams. Nucl. Instrum. Meth., A 281(3):640 - 648. 59, 91

Rink, A. (2008). Point-based ionizing radiation dosimetry using radiochromic materials and a fibreoptic readout system. PhD thesis, University of Toronto. 37

Robinson, M. (2003). Havar ${ }^{\circledR}$ a new, old Co-Cr biocompatible alloy for implants. Medical Device Materials: Proceedings of the Materials and Processes for Medical Devices Conference, Anaheim, California, USA, pages 324 - 328. ASM International. 143

Savitskii, E., Tylkina, M., Ipatova, S., and Pavlova, E. (1960). Properties of Tungsten-Rhenium alloys. Metallovedenie $i$ Term, HB No. 5489(9):483 - 486. 47

Schardt, D., Elsässer, T., and Schulz-Ertner, D. (2010). Heavy-ion tumor therapy: physical and radiobiological benefits. Rev. Mod. Phys., 82(1):383 - 425. xix, 21, 22

Schettino, G., Folkard, M., Vojnovic, B., Michette, A., and Prise, K. (2010). X-Ray Microbeams for Radiobiological Studies: Current Status and Future Challenges. In Progress, In Electromagnetics Research Symposium Proceedings. Xi'an, China: The Electromagnetics Academy, March, 6(3):56 - 60. 区ix

Schwab, T. (1991). Transport von schwerionen durch materie innerhalb ionenoptischer systeme. PhD thesis, University of Gieyen, Gieyen. GSI report 91-10. 10

Sesame team (2012). SESAME - Synchrotron-light for Experimental Science and Applications in the Middle East. Available at http://www.sesame.org.jo/sesame/index.php. Online, June. xxiii

Sesame team (2013). SESAME - Synchrotron-light for Experimental Science and Applications in the Middle East. Available at http://mag.digitalpc.co.uk/fvx/iop/esrf/sesamebrochure/. Online, June. Xxiii, Xxiv

Shikazono, N., Pearson, C., O'Neill, P., and Thacker, J. (2006). The roles of specific glycosylases in determining the mutagenic consequences of clustered DNA base damage. Nucleic Acids Res., 34(13):3722 - 3730. 15 
Soares, C. (2013). Radiochromic film. Lecture notes, United States department of commerce, national institute of standards and technology, Gaithersburg, MD 20899-0001. Available at http://www.aapm.org/default.asp. Online, November. 35

Soukup, M., Fippel, M., and Alber, M. (2005). A pencil beam algorithm for intensity modulated proton therapy derived from Monte Carlo simulations. Phys. Med. Biol., 50:5089 - 5104. 21]

Steel, G. (2007). Radiobiology of tumours. in Handbook of Radiotherapy Physics: Theory and Practice, 1st ed., P. Mayles, A. Nahum, and J. C. Rosenwald, Eds. New York: Taylor 8 Francis, ch. B7:127 - 148. xix

Sternglass, E. (1957). Theory of secondary electron emission by high-speed ions. Phys. Rev., 108(1):1 - 12. 46, 92, 93

Stolz, A. (2013). Diamond detectors for heavy ion beam tracking and timing measurements. NSCL, Michigan State University. Available at http://www.nscl.msu.edu. Online, November. 41, 43

Tapper, R. (2000). Diamond detectors in particle physics. Rep. Prog. Phys., 63:1273 - 1316. PII: S0034-4885(00)69857-3. Printed in the UK. 41,42

Ted Pella Inc., team (2014). Introducing the new standard in tissue slicing. Available at http://www.tedpella.com/easislicer.htm. 136

TI team (2013). LM231A/LM231/LM331A/LM331 precision voltage-tofrequency converters. TI - Texas Instruments. Available at http://www.ti.com. Online, June. 149

Tipler, P. and Llewellyn, R. (2008). Modern physics. Clancy Marshall, W. H. Freeman and Company. www.whfreeman.com, fifth edition. 46

Turner, J. (2007). Atoms, radiation, and radiation protection. $3^{\text {rd }}$ ed., WileyVCH, Germany. 12, 13

Varnasseri, S. (2005). The beam diagnostics for SESAME. Poster presentation, CAS, Trieste, Italy, October $2-14$. 区xv

Verbinski, V. and Burrus, W. (1969). Direct and compound-nucleus neutrons from $14-18 \mathrm{MeV}$ protons on ${ }^{9} \mathrm{Be},{ }^{14} \mathrm{~N},{ }^{27} \mathrm{Al},{ }^{56} \mathrm{Fe},{ }^{115} \mathrm{In},{ }^{181} \mathrm{Ta}$, and ${ }^{208} \mathrm{~Pb}$ and from $33-\mathrm{MeV}$ bremsstrahlung on ${ }^{27} \mathrm{Al},{ }^{206} \mathrm{~Pb},{ }^{208} \mathrm{~Pb}$, and ${ }^{209} \mathrm{Bi}$. Phys. Rev., 177(4):1671 - 1686. 66, 146, 147, 
Wang, L., Kielar, K., Mok, E., Hsu, A., Dieterich, S., et al. (2012). An end-toend examination of geometric accuracy of IGRT using a new digital accelerator equipped with onboard imaging system. Phys. Med. Biol., 57(3):757 - 769. 126, 127, 128, 129,

Warman, J., de Haas, M., Luthjens, L., Denkova, A., Kavatsyuk, O., et al. (2013). Fixed fluorescent images of an $80 \mathrm{MeV}$ proton pencil beam. Radiat. Phys. Chem., 85:179 - 181. 78

Wideł, M., Przybyszewski, W., and Rzeszowska-Wolny, J. (2009). Radiationinduced bystander effect: The important part of ionizing radiation response. Potential clinical implications. Hig Med Dosw, 63:377 - 388. 区x

Wille, C. (2011). Synchrotron radiation. Lecture notes, JUAS (Joint University Accelerator School), Archamps, France. 24

Wilson, R. (1946). Radiological use of fast protons. Radiology, 47(5):487 - 491. 3

Wolff, S. (1998). The adaptive response in radiobiology: Evolving insights and implications. Environ Health Persp, 106(1):277 - 283. xix

Yun, S., Hong, I., and Cho, Y. (2008). Development of the proton beam monitoring system at the $45-\mathrm{MeV}$ proton irradiation test facility. Journal of the Korean Physical Society, 25(3):783 - 788. 52, 59.

Zhao, L. and Das, I. (2010). Gafchromic EBT film dosimetry in proton beams. Phys. Med. Biol., 55(10):291 - 301. 36

Ziegler, J. (2013). Srim - the stopping and range of ions in matter. Available at http://www.srim.org/. 37, 80, 89, 91, 96, 109, 123

Zirkle, R., Marchbank, D., and Kuck, K. (1952). Exponential and sigmoid survival curves resulting from alpha and X irradiation of aspergillus spores. Journal of Cellular and Comparative Physiology, 39(S1):75 - 85. 


\section{Acknowledgements}

I had the opportunity of working in a very exciting science environment at LIP - Laboratório de Instrumentação e Física Experimental de Partículas and at the Department of Physics, University of Coimbra, during the last four years with very kind people. The team of LIP friends has been wonderful and I do believe that I have been a lucky $\mathrm{PhD}$ student, to have been involved in such a special environment.

Prof. Dr. Paulo Crespo has been the ideal thesis scientific supervisor. His permanent availability, sage advice, patient encouragement, and scientific guidance resulted in the writing of this thesis. I thank my academic supervisor Prof. Dr. Francisco Fraga whose steadfast support of this work was greatly needed and deeply appreciated.

My debt of gratitude must go to Prof. Dr. Rui Ferreira Marques from LIP and the Department of Physics, University of Coimbra. He patiently provided encouragement and useful advices along the years of the doctoral program.

I am grateful to Prof. Dr. Francisco Alves from ICNAS - Instituto de Ciências Nucleares Aplicadas à Saúde, University of Coimbra, for his invaluable support, helpful advices, and for making the experiments at ICNAS possible. A special acknowledgment is to Dr. Sérgio do Carmo from ICNAS, for his endless availability, effective discussions, and for the help and company during the beam time at ICNAS.

I want to express my sincere gratitude to Eng. Paulo Rachinhas from the Department of Radiotherapy of Coimbra University Hospital Center, for his precious and friendly collaboration and for the shared knowledge in the field of film dosimetry.

I am very thankful to Prof. Antero Abrunhosa from ICNAS and to Dr. Célia Gomes from IBILI - Institute for Biomedical Imaging and 
Life Sciences, University of Coimbra, for important discussions and sharing of ideas in the field of radiobiology. I acknowledge the support from Dr. Maria do Carmo Lopes, from Coimbra Institute of Oncology - IPOCFG, E.P.E., for providing several Gafchromic ${ }^{\circledR}$ EBT2 films.

I am also very grateful for the motivation and encouragement provided so generously by the staff of LIP (Eng. Rui Alves, Américo Pereira, Eng. Nuno Dias, Joaquim Oliveira, Carlos Silva, Dr. Alberto Blanco, Hugo Simões, Paulo Matins, Luís Pereira, Patrícia Cambraia Lopes, Dr. Miguel Couceiro, Dr. João Matins, Eng. Luís Lopes, Ricardo Caeiro, Elisabete Neves, and Teresa Marques).

I am very thankful to the Laboratory of Advanced Computing of the University of Coimbra, for the computing time provided on the milipeia HPC (high performance computing) cluster, without which it would have been almost impossible to carry out some parts of this work.

I thank Prof. Dr. José Mariano Gago and Prof. Dr. Paulo Fonte from LIP, for their encouragement and advice as I struggled to formulate the contents of this work. I also would like to thank Prof. Dr. Salman Salman from Al-Quds University, Palestine, for his advice and bibliographic help at the beginning of my thesis.

I acknowledge my funding sources, the FCT - Fundação para a Ciência e a Tecnologia, Lisbon, Portugal, from October 2010 to September 2014 through grant SFRH/BD/51139/2010, under a SESAME/FCT fund.

To all my friends, thank you for your understanding and encouragement. Your friendship makes life a wonderful experience.

Finally, I want to thank from the deep of my heart my parents, my sisters, my brothers, and my wife, who always believed in me and encouraged me with their love and support. 